Dies ist die kostenfreie PDF-Version des von Guido Kirsten, Magali Trautmann, Philipp Blum und Laura Katharina Mücke vom Französischen ins Deutsche übersetzten und herausgegebenen Buchs Kommunikationsräume von Roger Odin (Les espaces de communication - Introduction à la sémio-pragmatique).

Die Übersetzung ist 2019 bei oa books als hybride Open-AccessPublikation erschienen: https://www.oabooks.de/kommunikationsraeume. Sie ist sowohl als PDF als auch gedruckte Fassung über den self-publishing Verlag tredition (Paperback, Hardcover) zugänglich. Die Seitenzahlen stimmen überein.

\title{
Abstract
}

Mit Kommunikationsräume liegt erstmals ein Buch des französischen Film- und Medientheoretikers Roger Odin auf Deutsch vor. Es bietet eine Einführung in das Modell der Semiopragmatik, dessen Ausarbeitung sich Odin in den vergangenen Jahrzehnten gewidmet hat. Im Zentrum steht das Konzept des „Kommunikationsraums", mit dessen Hilfe erklärt werden kann, wie mediale Kommunikationen durch ihren Kontext reguliert werden. Außerdem erläutert Odin seine Idee verschiedener "Modi der Sinn- und Affektproduktion“, die auf unterschiedliche Filme, Bücher, Fernsehsendungen und andere kulturelle Artefakte angewandt werden können.

Attraktiv ist der semiopragmatische Ansatz für alle, die einen Ausweg suchen aus dem oft beobachteten Dilemma zwischen immanenten Theorien, die allein von,"Texten“ ausgehen, und rezeptionsseitig pragmatischen Modellen, die bei der "Aneignung" der Texte ansetzen. Die Semiopragmatik thematisiert dieses Dilemma und macht das Angebot einer spezifischen Verbindung der beiden Analyserichtungen. 


ROGER ODIN KOMMUNIKATIONSRÄUME 
Das Buch ist aus der Idee entstanden, die Semiopragmatik einer größeren Leserschaft im deutschsprachigen Raum zugänglich zu machen. Es handelt sich um eine Übersetzung von Roger Odins Einführung in seinen medientheoretischen Ansatz, der die produktive Aneignung von medialen Produktionen („Lektüremodi“) ins Zentrum rückt.

Der Autor Roger Odin promovierte 1975 mit einer semiotischen Analyse der Bilder von Jeanne d'Arc und habilitierte 1982 mit einer Arbeit zur Filmsemiologie. Von 1983 bis 2003 war er Professor für Informations- und Kommunikationswissenschaft an der Paris III Sorbonne Nouvelle. Er ist Autor von Cinéma et production du sens (1990), De la fiction (2000) und Les espaces de communication (2011) sowie Herausgeber von Bänden zum Dokumentar- und Familienfilm. Er zählt zu den wichtigsten französischen Medien- und Filmtheoretikern seiner Generation.

\section{Die Übersetzer*innen}

Guido Kirsten promovierte in Zürich mit einer Arbeit zum Realismus des Films und im Film, die stark von Odins Semiopragmatik inspiriert wurde (Filmischer Realismus, 2013). Anschließend arbeitete er als Postdoc in Stockholm und als Vertretungsprofessor in Mainz. Seit 2018 leitet er die Emmy Noether-Nachwuchsgruppe „Filmische Diskurse des Mangels“ an der Filmuniversität Babelsberg KONRAD WOLF (Potsdam).

Magali Trautmann promovierte in Bremen zum narrativen Kinodokumentarfilm und entwarf in ihrer Arbeit ein Modell zu dessen Analyse aufbauend auf Odins Semiopragmatik (Show and Tell, 2017). Parallel hierzu entwickelte und lehrte sie das Praxismodul Film im Master-Studiengang Transnationale Literaturwissenschaft: Literatur, Theater, Film (TnL). Von 2016 bis 2017 war sie wissenschaftliche Mitarbeiterin der TnL. Seit 2018 arbeitet sie als künstlerische Leiterin eines Kunstverlags und freie Autorin in Bremen.

Philipp Blum promovierte an der Philipps-Universität Marburg zum experimentellen Semidokumentarismus und baute methodisch auf dem semiopragmatischen Ansatz auf (Experimente zwischen Dokumentar- und Spielfilm, 2017). Von 2012 bis 2018 war er wissenschaftlicher Mitarbeiter im DFG-Forschungsprojekt zur Geschichte des dokumentarischen Films in Deutschland 1945-2005. Seit 2018 ist er Oberassistent am Seminar für Filmwissenschaft der Universität Zürich und Koordinator des NETZWERK CINEMA CH.

Laura Katharina Mücke ist Universitätsassistentin Prae Doc für die Professur „Theorie des Films“ an der Universität Wien. Sie arbeitet dort an ihrer Dissertation mit dem Arbeitstitel „Anti | Immersion. Zur Performativität filmischer Erfahrung zwischen Annäherung und Distanzierung“, die eine rezeptionsästhetische Verzahnung von Semiopragmatik und Filmphänomenologie anvisiert. Von 2016 bis 2019 war sie wissenschaftliche Mitarbeiterin im Fach Filmwissenschaft an der Universität Mainz. 


\section{ROGER ODIN KOMMUNIKATIONSRÄUME}

EINFÜHRUNG IN DIE SEMIOPRAGMATIK

od

Herausgegeben und aus dem Französischen übersetzt von Guido Kirsten, Magali Trautmann, Philipp Blum und Laura Katharina Mücke 
Übersetzt mit freundlicher Genehmigung von Presses universitaires de Grenoble

Titel der Originalausgabe:

Les espaces de communication. Introduction à la sémio-pragmatique

(C) 2011 Roger Odin

Bibliografische Information der Deutschen Nationalbibliothek:

Die Deutsche Nationalbibliothek verzeichnet diese Publikation in der Deutschen Nationalbibliografie; detaillierte bibliografische Daten sind im Internet über http://dnb.d-nb.de abrufbar.

(C) 2019 oa books, Berlin

Umschlag und typografische Beratung: Vera Rammelmeyer, mischen

Satz: Sarah-Mai Dang

Verlag und Druck: tredition GmbH, Halenreie 40-44, 22359 Hamburg

Der Text wird unter der Creative Commons-Lizenz CC BY-SA 4.0 veröffentlicht. Der Text darf beliebig geteilt, kopiert und weiter verwertet werden - vorausgesetzt er wird dabei ordnungsgemäß zitiert. Zudem muss der Text unter derselben Lizenz veröffentlicht werden. Diese Erlaubnis berührt nicht das Copyright Dritter, deren Material in dem Text benutzt wird. Für Details s. http://creativecommons.org/licenses/by-sa/4.0/.

Dieses Buch ist kostenfrei als PDF über die Website www.oabooks.de abrufbar. Dort findet sich auch eine vollständige Bibliografie der Schriften Roger Odins. 


\section{Inhalt}

Guido Kirsten und Frank Kessler:

„Ein Denken, das noch auf der Suche ist“

Grundzüge und Geschichte der Semiopragmatik 9

Vorwort der Übersetzerinnen und Übersetzer 25

Vorwort und Danksagung (zur französischen Ausgabe) 29

Einleitung: Das semiopragmatische „Modell“ 33

Kapitel 1

Kontext, Bedingungen und Kommunikationsraum

Kapitel 2

Der diskursive Raum

Kommunikative Kompetenz und Modi der Sinnproduktion

Kapitel 3

Ästhetischer Modus, künstlerischer Modus

Beziehungen zwischen Modi und Räumen

Kapitel 4

Kontextuelle Analyse und Kommunikationsraum

Der Kommunikationsraum des Familiengedächtnisses

Kapitel 5

Kommunikationsraum und Migration in neue Kontexte

Das Beispiel des Familienfilms

Kapitel 6

Textuelle Analyse und Semiopragmatik

Schluss

$\begin{array}{ll}\text { Liste der vorgeschlagenen Instrumente } & 179\end{array}$

Etappen der Konstruktion des semiopragmatischen „Modells“ 180

Literatur

Nachwort von Roger Odin zur deutschen Ausgabe:

Die Semiopragmatik heute 



\section{„Ein Denken,}

\section{das noch auf der Suche ist“" Grundzüge und Geschichte der Semiopragmatik}

Einleitung von Guido Kirsten und Frank Kessler

Anders als die meisten seiner Kolleginnen und Kollegen ist Roger Odin nicht über die klassische französische cinéphilie zur Filmwissenschaft gekommen, sondern über einen Amateurfilm-Club. Dort konnte er schon in den 1970er Jahren feststellen, dass im Bereich des film de famille die Regeln des Erzählkinos im Allgemeinen nicht gelten. Im Gegenteil: Wenn der Filmende - meist der Vater - versuchte, seine Filme stärker gemäß der in Amateurfilmhandbüchern beschriebenen Regeln zu gestalten, stieß̧en sie im familiären Kreis auf geringere Zustimmung (Odin 1979). Im Zuge seiner Studie zur Rhetorik des Familienfilms kommt Odin zu dem für die Semiopragmatik grundlegenden Schluss, dass es unmöglich sei, „sich den Texten (in diesem Fall den filmischen Texten) über einen rein immanenten Ansatz zu nähern“; man müsse daher „eine konsequent pragmatische Perspektive einnehmen, um zu begreifen, auf welche Weise sie Bedeutung erzeugen“ (ibid., 368).

Mit dem Amateur- und Familienfilm hat sich Odin auch später noch ausführlich beschäftigt, unter anderem im Rahmen eines internationalen Forschungsprojekts in den 1990er Jahren (Odin 1995a). ${ }^{1}$ Der Platz, den er diesem quantitativ bedeutsamen, vor ihm jedoch wenig bearbeiteten Bereich der Filmproduktion eingeräumt und sein Stellenwert in der Filmwissenschaft, zu dem er so beigetragen hat, gehören zu Odins bleibenden Meriten.

1 Auf dieses Projekt folgte ein ebenfalls von Odin initiiertes Forschungsprojekt zum „goldenen Zeitalter“ des europäischen Dokumentarfilms (Odin 1998a). 
Wie für andere Phänomene auch, lässt die chronologische Lektüre seiner Schriften erkennen, wie sich sein Denken zum Familienfilm entwickelt hat. Der letzte Stand findet sich im vorliegenden Buch (in den Kapiteln 4 und 5). Ein entscheidender Gedanke ist dort, dass der Familienfilm - anders als Spiel- und Dokumentarfilm - nicht auf einen Lektüremodus hin angelegt ist, in dem er besonders gut funktioniert, sondern auf zwei verschiedene, die wiederum kombiniert werden können: den privaten und den intimen Modus, die sich insbesondere durch die Kollektivität resp. Individualität der Sinn- und Affektproduktion voneinander unterscheiden. Das schließt gleichwohl nicht aus, Familienfilme (insbesondere, wenn es sich nicht um die der eigenen Familie handelt) weder im einen noch im anderen Modus zu lesen, sondern dokumentarisierend: sie als historische Dokumente (etwa der Ideologie des Familientums) zu betrachten (Odin 2008). ${ }^{2}$

Dass Filme ihre Lektüre und entsprechend ihre Bedeutung nicht determinieren und daher nicht allein über ihre semiotische Binnenstruktur zu verstehen sind, ist die zentrale Erkenntnis Odins und der Ausgangspunkt seiner Theoriebildung. Gleichzeitig hat er an den theoretischen und terminologischen Errungenschaften der klassischen Filmsemiologie von Christian Metz stets festgehalten. So war er bemüht, beide Einsichten in einem Entwurf $\mathrm{zu}$ kombinieren: einerseits, dass das semiotische Instrumentarium und Vokabular es ermöglicht, Kommunikate präzise aufzuschlüsseln; andererseits, dass die Bedeutungszuschreibung immer und grundsätzlich kontextabhängig ist. Die Semiopragmatik zielt also darauf ab, die textimmanente Perspektive der strukturalistischen Semiotik hinter sich (jedoch nicht außer Acht) zu lassen und den Einfluss des Kontexts auf die Bedeutungsproduktion theoretisch und analytisch fassbar $\mathrm{zu}$ machen.

2 Darüber hinaus können Familienfilme auch allgemein als historische Quellen für eine „Geschichte von unten“ betrachtet werden; vgl. z.B. Roepke 2001. Odin (2003) analysiert seinerseits das Verhältnis von Familienfilmen und Geschichtsschreibung anhand von Péter Forgács' Film A BARTOS CSALÁd (DiE FAMILIE BARTOS, HUN 1988), Teil einer umfangreichen Serie von Arbeiten, in denen der Filmemacher die ungarische Geschichte im Found Footage-Modus darstellt; vgl. auch die Ausführungen dazu im vorliegenden Buch, S.142-146. 


\section{Zur Geschichte eines Work-in-Progress}

Odins erste Buchveröffentlichung aus dem Jahr 1977 trägt den Titel Dix années d'analyses textuelles de films: bibliographie analytique - und hält, was er verspricht: eine ausführliche, kommentierte Bibliografie filmwissenschaftlicher Forschungsliteratur, die sich im weitesten Sinn als „textuelle Analyse“ verstehen lässt (Blüher/Kessler/Tröhler 1999). Metz schreibt im Geleitwort, dass er sich lange eine solche Publikation gewünscht habe. Nun sei er dankbar, dass jemand - was selten sei Geschmack an derartiger Arbeit finde und darüber hinaus - was noch seltener sei - die notwendigen Qualitäten mitbringe (Metz 1977, 11). Neben Innovation, Systematik und Offenheit für Neues, die Odins Theorie insgesamt prägen, zeigen sich an diesem Werk auch seine Tugenden als neugieriger Leser und gewissenhafter Fleißarbeiter.

In den späten 1970ern beginnt Odin die Arbeit an dem Entwurf seiner semiopragmatischen Theorie. 1982 schließt er seine Habilitation ab, die den Titel L'analyse sémiologique des films: Vers une sémiopragmatique du cinéma trägt und von Metz betreut wurde. In der Jury der Disputation saßen neben Metz unter anderem der Semiotiker Algirdas Julien Greimas sowie Michel Colin, der seinerseits eine andersgeartete Reformulierung und Neukonstituierung der Filmsemiologie anstrebte (Colin 1985; 1992). Einstimmig entschied sich die Jury unter dem Vorsitz des Linguisten Jean-Claude Chevalier für die höchste Auszeichnung („mention très honorable“). Im 1983 in der ersten Ausgabe der Zeitschrift Iris veröffentlichten Artikel „Pour une sémio-pragmatique du cinéma“ fasst Odin die wichtigsten Ergebnisse seiner Habilitationsschrift zusammen und formuliert die Programmatik seines Theorieprojekts. An Metz anknüpfend, den Odin stets als seinen wichtigsten Lehrer betrachtete (Odin 2018), nennt er als grundlegende Ziele der Filmsemiologie: erstens zu verstehen, wie Filme verstanden werden (nach Metz' Formulierung ,comprendre comment le film est compris“) und zweitens zu verstehen, wie die affektive Beziehung zwischen Film und Zuschauer funktioniert. Odin ist überzeugt, dass sich beide Fragen nur befriedigend beantworten lassen, wenn „die Pragmatik das Kommando übernimmt“ (Odin 1983a, 67).

In diesem Text zeigt sich bereits ein Zug, der Odins Theorie bis heute prägt: ein Hang zur Abstraktion und zur Bildung rein „heuris- 
tischer" Kategorien. So spricht er nicht von empirischen Zuschauern, sondern von dem Zuschauer, den er als „konstruierte Entität“, als „Aktant“ begreift (ein Begriff, den er aus Greimas“ Theorie übernimmt und der eine Position innerhalb einer Struktur bezeichnet) und als „Schnittpunkt eines Bündels von Determinanten“ definiert. Der so verstandene Zuschauer ergibt sich aus der jeweiligen Institution, innerhalb derer er in der Rezeption agiert, und die Odin ihrerseits als Struktur begreift, die ein „Bündel an Determinanten artikuliert“ (ibid., 70). ${ }^{3}$ Hier (und in seinem gesamten späteren Werk) ist Odin stark darum bemüht, dem Missverständnis vorzubeugen, dass sich seine Aussagen auf eine unmittelbar gegebene Wirklichkeit bezögen und an dieser ohne Weiteres überprüft werden könnten. Zwar wünscht er sich später ausdrücklich eine Verbindung mit empirischen Ansätzen, selbst leistet die Semiopragmatik die Empirie jedoch nicht und sie liefert dafür auch keine Methodologie. Mit seiner Annahme, die Räume der Textproduktion und -rezeption seien radikal geschieden - die Semiopragmatik sei daher eine Theorie der Nicht-Kommunikation (2019 [in diesem Band], 44) - möchte Odin vor allem Fragen aufwerfen, die ohne sie nicht formuliert werden könnten. ${ }^{4}$

Als zentral hat sich für solche Fragen zunehmend ein Aspekt erwiesen, der in der Programmatik von 1983 noch nicht explizit genannt wird: die Modi der Sinn- und Affektproduktion. Dieser Aspekt rückt ein Jahr später - wenn auch noch nicht unter dieser Bezeichnung - mit dem Artikel „Film documentaire, lecture documentarisante“ in den Fokus. Im deutschsprachigen Raum gehört er zu den bekanntesten Texten Odins, da er früh übersetzt und in zwei Anthologien aufgenommen wurde (Odin 1990 [1984]; Odin 1998b [1984]). Eingangs konstatiert Odin hier, dass der Dokumentarfilm sich nicht über ihm

3 Eine ausführliche und anspruchsvolle Überlegung dazu, was der Begriff des Filmzuschauers (im Sinne eines theoretischen Konstrukts) bedeutet, liefert Odin 1992 mit seinem Artikel „Le spectateur du cinéma: approche sémio-pragmatique“.

4 In diesem Punkt ähnelt Odins Denken dem von Niklas Luhmann, der verschiedentlich betonte, aus heuristischen Gründen von der „Unwahrscheinlichkeit der Kommunikation“ auszugehen: „Kommunikation ist unwahrscheinlich. Sie ist unwahrscheinlich, auch wenn wir sie jeden Tag erleben, sie praktizieren und ohne sie nicht leben würden. Diese unsichtbar gewordene Unwahrscheinlichkeit gilt es vorab zu begreifen, und dazu bedarf es einer sozusagen contra-phänomenologischen Anstrengung“ (Luhmann 2001 [1981], 78). 
immanente Eigenschaften definieren lasse. Angesichts dieser Unmöglichkeit argumentiert Odin für einen radikalen Perspektivwechsel: Nicht das dokumentarische Werkensemble solle charakterisiert werden, sondern die dokumentarisierende Lektüre, die dann wiederum das Ensemble definieren könne (insofern die Werke, die es konstituieren, auf diese Art der Lektüre angelegt seien).

Beim Lesen des Artikels ist allerdings $\mathrm{zu}$ bedenken, dass Odins Theorie, seine Terminologie und vor allem die Differenzierung der Modi ein Work-in-Progress waren und sind, wie er selbst im zehn Jahre später erschienenen „Semio-pragmatique du cinema et de l'audiovisuel. Modes et institutions“ schreibt (1994, 33). Dies betrifft etwa die Abgrenzung der dokumentarisierenden Lektüre von der fiktionalisierenden, die er 1984 noch (und dies kann heute zu Missverständnissen führen) als fiktivisierende Lektüre bezeichnete. Seitdem hat Odin eine wichtige Unterscheidung zwischen Fiktionalisierung und Fiktivisierung eingeführt: Während erstere die spezifisch artikulierte Gesamtheit der Operationen meint (zu denen unter anderem auch Diegetisierung und Narrativierung zählen), bezeichnet letztere nur eine davon, nämlich die der Irrealisierung der Äußerungsinstanz, „den Prozess, durch den der Leser einen fiktiven Enunziator konstruiert“ (Odin 1996, 13). ${ }^{5}$

Die Zahl der Modi der Sinn- und Affektproduktion ist über den Lauf der Zeit gewachsen, aber manche Modi sind auch wieder verschwunden oder wurden umbenannt. 1994 unterscheidet er acht: mode spectaculaire, mode fictionnalisant, mode énergétique, mode privé, mode documentaire, mode argumentatif/persuasif, mode artistique und mode esthétique (1994, 34ff). ${ }^{6}$ In Kommunikationsräume hat sich der

5 Tatsächlich ist die Sache noch komplizierter: In „Christian Metz et la fiction“ (1996) zeigt Odin, dass Fiktion bei Metz drei verschiedene Sachverhalte bezeichnet. Nur die dritte (von Odin „fictivisation 3“ genannt) bezeichnet die Fiktivisierung des Enunziators resp. dessen Situierung in einem „Anderswo, das weder unsere wirkliche Welt noch die des Films (die Diegese) ist“ (ibid., 13). Die anderen beiden Metz’schen „Fiktivisierungen“ sind an die Medialität des Films - den sogenannten „imaginären Signifikanten“ - resp. an die Narration gebunden und tauchen daher an anderen Stellen (und unter anderen Bezeichnungen) in Odins System auf (ibid., 16).

$6 \mathrm{Zu}$ seinem Vorschlag einer Differenzierung zwischen künstlerischem und ästhetischem Modus hat sich Odin erstmals 2002 in seinem Aufsatz „Kunst und Ästhetik bei Film und Fernsehen“ ausführlich geäußert (Odin 2002). 
argumentative/überzeugende Modus im „diskursiven Modus“ aufgelöst. Hinzugekommen ist - bereits 2000 in De la fiction, vor dem Hintergund einer eingehenden Beschäftigung mit Norman McLarens Film NeIGHBOURS (CAN 1952), die 1985 begann (Odin 1985) - der „fabularisierende“ Modus. Zudem spricht Odin von einem „Modus der Authentizität“ und einem „Modus der Zeugenschaft“. Neuerdings kennt Odin noch einen „Modus des Making Of“ und er scheint die Vorschläge anderer Forscherinnen und Forscher, einen „Modus des Pastiche“ sowie einen „hermeneutischen Modus“ zu konstruieren, wohlwollend aufzunehmen (2019 [in diesem Band], 193-198). Um im Sinne Odins als Modus der Sinn- und Affektproduktion gelten zu können, muss es sich um (1.) spezifische, wiederholbare Kombinationen kognitiver Operationen handeln, die (2.) auf verschiedene Arten von Werken anwendbar sind, die (3.) „,in den Zuschauern“ als aktivierbare Prozesse vorhanden und aktivierbar sind und (4.) gemeinsam die spezifische kommunikative Kompetenz eines Zuschauers konstituieren.

Theoriearbeit ist für Odin in erster Linie Arbeit an Begriffen sowie an deren Stellenwert in seinem System. Üblicherweise bringt er ein Konzept zunächst ein und verschiebt dann in der Folge (in späteren Aufsätzen, in denen er darauf zurückkommt) dessen Position oder ergänzt es um ein qualifizierendes Adjektiv. So erläutert er etwa das Konzept der Mise en phase erstmals 1983 in einem Aufsatz zu Jean Epstein Le Tempestaire (F 1947). Dort definiert er es als Film/ Zuschauer-Relation, die den Relationen in der Diegese entspricht: Mit einer Mise en phase haben wir es zu tun, wenn die filmischen Mittel (in Epsteins Werk etwa Beschleunigungen und Verlangsamungen der Bilder des Meeres) als homolog zum gerade Erzählten aufgefasst werden (zu dem Wirken des titelgebenden „Sturmbändigers“). Solange dies gelingt, ist die Zuschauerposition „homogenisiert“ und folgt den narrativen Dynamiken in der Diegese, was laut Odin „charakteristisch für die Funktionsweise des fiktionalen Films“ sei, also „einen der Operatoren des Fiktionseffekts“ darstelle (1983b, 224f). ${ }^{7}$

7 Der Witz an LE TEMPESTAIRE ist für Odin allerdings, dass der Film die Mise en phase immer wieder sabotiert und zu dem führt, was er eine „déphasage“ nennt. (Bildlich kann man sich eine solche Phasenverschiebung so vorstellen, dass sich die Linie der mentalen 
Elf Jahre später kommt Odin auf das Konzept zurück, ergänzt jedoch, dass er es nunmehr vorziehe, von „fiktionalisierender Mise en phase“ zu sprechen und diese als „Mitschwingen im Rhythmus der erzählten Ereignisse“ zu fassen, um sie von anderen Arten der Mise en phase zu unterscheiden, etwa der ,energetischen Mise en phase“, bei der im Rhythmus der Bilder und Töne mitgeschwungen wird (1994, 35; Herv.i.O.). Außerdem schreibt er:

Im Übrigen haben wir unsere Meinung in einem wichtigen Punkt geändert: Wir glauben nun nicht mehr, dass die „Mise en phase“ eine Operation unter anderen innerhalb des fiktionalisierenden Modus darstellt; wir betrachten sie vielmehr als das Ergebnis der Kombination der verschiedenen Operationen, die für diesen Modus konstitutiv sind: die „fiktionalisierende Mise en phase“ ist nichts anderes als der Fiktionseffekt selbst (ibid.; Herv. i.O.).

Allerdings ist diese Meinungsänderung nicht von langer Dauer: In De la fiction versteht Odin die Mise en phase wieder als eine von mehreren (nun sieben) Operationen oder (wie es dort auch heißt) „Prozessen“ $(2000,64)$. Bei diesem Verständnis ist es auch in Kommunikationsräume geblieben.

Wer genau liest, erkennt viele derartige Veränderungen und kleine Verschiebungen in Odins Konzeptionen. Manche hat er markiert und als bedeutsam angezeigt, andere unter der Hand vollzogen (als sei er sich selbst nicht immer sicher). Man versteht so besser, was er meint, wenn er im Vorwort zu Kommunikationsräume schreibt, er habe sich lange gesträubt, ein Buch über die Semiopragmatik zu verfassen, da ihm Artikel „geschmeidiger“ erschienen, besser passend „zu einem Denken, das noch auf der Suche ist“ (2019 [in diesem Band], 29).

Zuschaueraktivität von der audiovisuellen Linie des Films trennt, während beide in der Mise en phase gleich getaktet sind.) Da er die fiktionalisierende Lektüre einerseits anregt, andererseits immer wieder blockiert, muss LE TEMPESTAIRE entweder als „schlecht gemachte Fiktion“ (,un film de fiction mal fait“) betrachtet werden oder als Film, der eigentlich auf eine andere Lektüre angelegt ist: eine Lektüre, die von der Fiktion ausgehend zu einem diskursiven Verständnis des Films gelangt, zu einer Lektüre „als Diskurs über das Kino“ (Odin 1983b, 235; Odin 2000, 119; Herv. i.O.). 
Die Entwicklung von Odins Denken lässt sich auch an dem epistemischen Abstand ablesen, den die vergleichende Lektüre von „L'entrée du spectateur dans la fiction“ (1980) und „Der Eintritt des Zuschauers in die Fiktion“ (2006 [2000]) offenbart. Im Text von 1980 ist das Agens des Eintretens noch der filmische Text, genauer das dynamische Arrangement von diegetischen und nicht-diegetischen Elementen: Der Anfang von PARTIE DE CAMPAGNE (Jean Renoir, F 1936) sei dadurch gekennzeichnet, dass auf der Bild- wie auch auf der Tonebene jene Elemente kontinuierlich zunehmen, die zur filmischen Welt gehören, in deren Zusammenhänge der Film die Zuschauer verwickelt: „So sichert der Film, Schritt für Schritt, den Eintritt des Zuschauers in die Fiktion“ $(1980,213)$.

Zwanzig Jahre später, im siebten Kapitel von De la fiction, das die Grundlage des 2006 auf Deutsch erschienenen Aufsatzes darstellt, hat sich, im Einklang mit Odins gesamter Theorieentwicklung, das Agens vom Film auf den Zuschauer verschoben: Nun ist nicht mehr von diegetischen Bildern und Tönen die Rede, sondern von der Konstruktion einer Diegese, an der die Zuschauer aktiv beteiligt sind. Obwohl das unmittelbare Objekt von Odins Analyse auf den ersten Blick identisch ist - die ersten zehn Einstellungen von PARTIE DE CAMPAGNE $^{8}$ - und der ältere offenkundig die Basis des jüngeren Textes lieferte, sind die theoretischen Grundannahmen doch nicht mehr dieselben. Fast alle Umformulierungen in der neuen Fassung zeigen an, dass Odin nicht mehr einen Eintritt in eine gegebene Welt - eine Positionierung, Ausrichtung und Implikation des Zuschauers durch den Film - zu fassen versucht, sondern den konstruktiven Aufbau dieser Welt im Zuge der Lektüre: die Diegetisierung. Wenn Odin es auch nicht in aller Deutlichkeit markiert (und einzelne, in beiden Texten gleich oder ähnlich lautende Sätze es tendenziell verschleiern) - im Grunde hat sich der Gegenstand der Analyse gewandelt: vom Textstück (dem Aufbau des Filmanfangs) zur mentalen Operation der Diegetisierung.

8 In De la fiction setzt Odin im achten Kapitel die Analyse mit den Einstellungen 11 bis 31 fort (Gegenstand ist nunmehr die Narrativierung), bevor er am selben Beispiel im neunten Kapitel die Beziehungen zwischen diegetischen und filmischen Relationen sowie im zehnten die Dynamik der Mise en phase erläutert (2000, 83-111). 
Hinzukommt, dass manche Ideen und Begriffe bei Odin bisweilen gewisse Latenzen besitzen. Sie werden zunächst beiläufig erwähnt und erst später theoretisch entfaltet. Das betrifft etwa den zentralen Begriff des vorliegenden Buchs, den des Kommunikationsraums: Schon 1989 kündigte Odin in dem Aufsatz „A Semio-pragmatic Approach of the Documentary Film“ ein nicht realisiertes, zweibändiges Buchprojekt Pour une sémio-pragmatique du cinéma an, dessen erster Band den Titel L'espace de la communication fictionnelle tragen sollte; der zweite sollte L'espace de la communication documentarisante heißen (Odin 1989, 93 u. 96). Im überarbeiteten Nachdruck dieses Aufsatzes von 1995 ist davon nicht mehr die Rede, angekündigt wird hier nur noch „my forthcoming Cinéma et effet fiction“ (1995, 234). Weitere fünf Jahre später hat dieses Buch endlich das Licht der Welt erblickt, nunmehr De la fiction getauft - aber der Begriff des Kommunikationsraums wird darin nicht thematisiert.

In Kommunikationsräume ersetzt der Begriff den der Institution, der in Odins Schriften der 1980er und 90er als für die Bedeutungsproduktion während der Filmsichtung entscheidend betrachtet worden war, jedoch das Risiko barg, gedanklich mit tatsächlichen gesellschaftlichen Institutionen assoziiert zu werden (Odin 2019 [in diesem Band], 65) und damit über den selbstgesteckten Rahmen der Semiopragmatik hinauszugehen (wie Francesco Casetti einmal in einer Diskussion bemerkte). ${ }^{9}$ Ein „Kommunikationsraum“ ist dagegen eine dezidiert theoretische Konstruktion, die es erlauben soll, die für die Bedeutungsproduktion in verschiedenen Kontexten relevanten Faktoren zu benennen und sie in ihren Funktionen zu beschreiben.

\footnotetext{
9 Zumal Odin den Begriff der Institution bisweilen selbst im konkreteren Sinn verwendete. So behandelt er in dem genannten Aufsatz zum dokumentarischen Film drei sehr unterschiedliche Institutionen, die jeweils, wenn auch auf verschiedene Weise, ihren Adressaten einen „dokumentarisierenden Pakt“ anbieten: die Schule, die Bibliothèque Publique d'Information im Centre Georges Pompidou sowie das Fernsehen (Odin 1989). Man erkennt schon an der Heterogenität dieser Liste, wie mehrdeutig der Begriff verwendet werden kann.
} 


\section{Das Verhältnis zur „semio-linguistischen“ Filmtheorie}

Bemerkenswert an Odins Werkbiografie ist, dass er seine erste Monografie nach der Habilitation nicht einer Darlegung der Semiopragmatik widmete, sondern mit Cinéma et production du sens 1990 eine Bilanz der „semio-linguistischen“ Filmtheorie, also der vor allem von Metz geprägten Semiologie vorgelegt hat. Die Semiopragmatik kommt nur im Ausblick auf den letzten Seiten kurz zur Sprache. Das Buch, das in einer von Odin selbst betreuten Reihe erschien, die sich vor allem an Studierende wendet, hat das erklärte Ziel, anhand der Untersuchung einiger klar umrissener Fragen darzulegen, wie die semio-linguistische Arbeit funktioniert (Odin 1990, 14). Es basiert auf einer genauen und umfassenden Kenntnis dieser Theorietradition. ${ }^{10}$

$\mathrm{Zu}$ den semio-linguistischen Fragen, die Odin in Cinéma et production du sens behandelt, gehören im ersten Teil der Vergleich mit den verbalen Sprachen hinsichtlich der sogenannten „zweifachen Gliederung“ (also der Gliederung eines Textes in bedeutungstragende Einheiten und deren Gliederung wiederum in eine begrenzte Zahl von Lauteinheiten, die selbst keine Bedeutung vermitteln), die Unterscheidungen zwischen paradigmatischen und syntagmatischen Beziehungen sowie zwischen Denotation und Konnotation. Im zweiten Teil erläutert Odin die Begriffe der filmischen und kinematografischen „Codes“ und „Sub-Codes“, bevor er sich im dritten Teil drei speziellen Problemen widmet: dem des vermeintlichen Ähnlichkeitsverhältnisses von filmischem Bild und Wirklichkeit („Analogie“, „Realitätseindruck“), dem der Metz‘schen „Großen Syntagmatik“ und der filmischen Diskursivität sowie dem der Bild-Ton-Relationen. Insgesamt belegt das Buch das enorm hohe und international einmalige Differenzierungs- und

10 In Frankreich gab es bis in die 1970er vor allem zwei Linien in der strukturalistischen Semiotik: Zum einen die von Algirdas Greimas begründete Schule, die ihre Disziplin als sémiotique bezeichnete, zum anderen die von Ferdinand de Saussure über Claude LéviStrauss zu Roland Barthes führende, die an der Bezeichnung sémiologie festhielt. Für die Metz'sche Filmsemiologie war die zweite Linie wichtiger (Metz betrachtete Barthes als seinen wichtigsten „Lehrer“), an einigen Stellen bediente er sich aber auch bei Ideen aus Greimas' strukturaler Semantik. (Zur Genese von Metz' Denken vgl. Tröhler/Kirsten 2018.) Odin war Teil der Schule von Greimas, bevor er zu Metz wechselte. Er kennt daher beide Theorielinien sehr gut. 
Reflexionsniveau der französischen Filmwissenschaft zu diesem Zeitpunkt. (All jenen, die meinen, den semiologischen Ansatz mit ein paar simplen Überlegungen abtun zu können, sei seine Lektüre als Korrektiv dringend ans Herz gelegt.)

Andererseits scheint Odins Fixierung auf die französische Tradition eine eingehendere Beschäftigung mit alternativen Semiotikkonzeptionen blockiert $\mathrm{zu}$ haben. Insbesondere die Auseinandersetzung mit Charles S. Peirce ist bei ihm oberflächlich geblieben (ibid., 11ff). Dabei sind gerade dessen Grundannahmen mit denen der Semiopragmatik eigentlich verwandt und Peirce hätte der Semiopragmatik liefern können, was ihr bis heute erstaunlicherweise fehlt: ein Zeichenbegriff (ein Mangel, den Odin übrigens direkt von Metz übernommen hat ${ }^{11}$ ). Wünschenswert wäre eigentlich eine Synthese aus Peirce, Metz, Colin und Odin, zu der es jedoch - auch aus wissenschaftskonjunkturellen Gründen: Filmsemiotik wird fast nur noch von Außenseitern betrieben und stößt in der scientific community weitgehend auf Desinteresse bislang nicht gekommen ist.

\section{Ausblick}

Eine Verbindung von Semiotik und Pragmatik hat im Bereich der Filmund Medienwissenschaft nicht nur Roger Odin vorgeschlagen: JeanPierre Meunier und Daniel Peraya haben ihrer 1993 erschienenen Einführung in die Kommunikationstheorie den Untertitel „Analyse semio-pragmatique de la communication médiatique“ gegeben; ${ }^{12}$ Hans J. Wulff nennt den von ihm in seiner Habilitationsschrift Darstellen und Mitteilen vorgelegten Ansatz eine „Pragmasemiotik“ (1999); und auch Rick Altmans Genretheorie, deren syntaktische und semantische

11 Metz hat sich dezidiert gegen das Konzept des filmischen Zeichens ausgesprochen (1973 [1971], 222ff), obwohl er wiederholt mit den Termini „Signifikant“ und „Signifikat“ operiert. 12 Auch die Übereinstimmungen mit älteren Theorien von Jean-Pierre Meunier, die Odin scheinbar nicht kannte, sind bemerkenswert. In Les structures de l'expérience filmique (2019 [1969]) unterscheidet Meunier zwischen drei phänomenologisch grundsätzlich verschiedenen Attitüden, die drei unterschiedlichen Objekttypen Film entsprechen: home movie attitude (,l'attitude-souvenir“), documentary attitude („l'attitude-documentaire“) und fiction attitude (,l'attitude-fiction“). 
Ebene (Altman 1999b [1984]) er in der Monografie Film/Genre (1999) um die der Pragmatik erweiterte, ließe sich als semiopragmatischer Ansatz begreifen.

Keiner der Genannten - die sich im Übrigen weder aufeinander noch auf Odin beziehen - hat jedoch den Ansatz zu einer veritablen Theorie ausgearbeitet. Für Odin hingegen ist die Semiopragmatik, an der er mittlerweile seit mehr als vierzig Jahren arbeitet, zum medientheoretischen Lebenswerk geworden. Über die meiste Zeit hat er dieses praktisch als Ein-Mann-Unternehmen betrieben (trotz gelegentlicher Kooperationen etwa mit Casetti). Die theoretische und begriffliche Entwicklung des Ansatzes lag und liegt allein bei Odin. Das hat auch damit zu tun, dass er es - wie Metz und im Unterschied zu Greimas nie darauf angelegt hat, eine Schule zu begründen. Dies ist möglicherweise einem Zug ostentativer Bescheidenheit geschuldet, könnte aber auch damit zu tun haben, dass Odin die Kontrolle über seine Theorie behalten wollte. (Im Nachwort zum vorliegenden Buch zeigt sich seine Neigung, die Vorschläge jüngerer Kolleginnen und Kollegen, die sich des Ansatzes bedienen, in seinem Sinn zu korrigieren. Odin ist einerseits erfreut, dass sich eine jüngere Generation seiner Theorie bedient; andererseits ist er um deren Kohärenz besorgt.)

Wie dem auch sei: Die vorliegende Übersetzung von Odins vorerst finalem Zwischenstand seiner Modellbildung kann als Einladung verstanden werden, sich der Semiopragmatik zu bedienen, ihre heuristische Produktivität zu nutzen, sich aber auch vertiefend und kritisch mit ihr auseinanderzusetzen. ${ }^{13}$ Konsequenterweise versteht Odin seine pragmatische Theorie selbst pragmatisch: als Werkzeugkasten, in dem verschiedene spezielle Instrumente bereitliegen, mittels derer besondere Perspektiven auf medial vermittelte Kommunikationsakte zu gewinnen sind. Die Instrumente können auf neue Gegenstände angewandt und in ihrer Anwendung angepasst, geschärft,

13 Seit dem Erscheinen von Les espaces de communication hat sich Odin nicht mehr mit den theoretischen Grundlagen der Semiopragmatik beschäftigt. Sein Hauptaugenmerk galt in den vergangenen Jahren der neuartigen audiovisuellen Bewegtbildkommunikation mittels Smartphones (Odin 2018 [2012]) sowie der Filmrezeption über Smartphones (Odin 2016). Diese Beschäftigung erweitert allerdings wiederum das Feld der Semiopragmatik, wie er im Nachwort dieses Buchs erläutert. 
verfeinert werden. Es kann sich bei genauerer Prüfung aber auch erweisen, dass manche begrifflichen Werkzeuge von Grund auf neu entworfen werden oder schon die Methoden ihrer Bildung selbst nachjustiert werden müssten. $\mathrm{Zu}$ wünschen ist Odins Theorie eine anspruchsvolle Diskussion und eine gründliche Weiterentwicklung: dass der Arbeitsprozess in progress als kollektiver fortgesetzt werde; dass das Denken weiter auf der Suche bleibe ... 


\section{Literatur}

Altman, Rick (1999a): Film/Genre, London: British Film Institute.

— (1999b): A Semantic/Syntactic Approach to Film Genre [1984]. In: Altman 1999a, S. 216225.

Colin, Michel (1985): Langue, film, discours: Prolégomènes à une sémiologie générative du film. Paris: Klincksieck.

- (1992): Cinéma, télévision, cognition. Nancy: Presses universitaires de Nancy.

Hanich, Julian / Fairfax, Daniel (Hg.) (2019): The Structures of the Film Experience by Jean Pierre Meunier: Historical Assessments and Phenomenological Expansions. Amsterdam: Amsterdam University Press.

Luhmann, Niklas (2001): Die Unwahrscheinlichkeit der Kommunikation [1981]. In: Ders., Aufsätze und Reden. Hg v. Oliver Jahraus. Stuttgart: Reclam, S. 76-93.

Metz, Christian (1973): Sprache und Film [frz. 1971]. Frankfurt a.M.: Athenäum.

- (1977): Préface. In: Odin 1977, S. 7-11.

Meunier, Jean-Pierre / Peraya, Daniel (1993): Introduction aux théories de la communication. Analyse sémio-pragmatique de la communication médiatique. Brüssel: DeBoeck.

Odin, Roger (1977): Dix années d'analyses textuelles de films: bibliographie analytique. Lyon: Centre de récherches linguistiques et sémiologiques de Lyon.

- (1979): Rhétorique du film de famille. In: Rhétoriques, sémiotiques (Revue d'Esthétique 12/1979). Paris: UGE 10/18, S. 340-373.

- (1980): L'entrée du spectateur dans la fiction. In: Jacques Aumont \& Jean-Louis Leutrat (Hg.), Théorie du film. Paris: Albatros, S. 198-213.

- (1982): L'analyse semiologique des films. Vers une sémio-pragmatique [3 Bände]. Doctorat d'État [Habilitationsschrift], EHESS (Paris).

- (1983a): Pour une sémio-pragmatique du cinéma. In: Iris 1,1, S. 67-82.

- (1983b): Mise en phase, déphasage et performativité dans LE TEMPESTAIRE de Jean Epstein. In: Communications 38, S. 213-238.

— (1984): Film documentaire, lecture documentarisante. In: Jean-Charles Lyant \& Roger Odin (Hg.), Cinémas et Réalités. Lyon: CIEREC, S. 263-278.

— (1985): Neighbours di Norman MacLaren. Un film-favola. In: Carte Semiotiche 1, S. 27-39.

- (1988): Du spectateur ficitionnalisant au nouveau spectateur: approche sémiopragmatique. In: Iris 5,1 (=8), S. 121-139.

- (1989): A Semio-pragmatic Approach of the Documentary. In: Willem De Greef \& Willem Hesling (Hg.), Image, Reality, Spectator. Essays on Documentary Film and Television. Leuven, Amersfoort: Acco, S. 91-100.

— (1990): Dokumentarischer Film - dokumentarisierende Lektüre [frz. 1984]. In: Christa Blümlinger (Hg.), Sprung im Spiegel: Filmisches Wahrnehmen zwischen Fiktion und Wirklichkeit. Wien: Sonderzahl, S. 125-146.

- (1992): Le spectateur du cinéma: approche sémio-pragmatique. In: Communication: Information Médias Théories 13,2, S. 38-58.

- (Hg.) (1995), Le film de famille. Usage privé, usage public: Paris: Méridiens Klincksieck.

- (1996): A Semio-pragmatic Approach to the Documentary Film [1989]. In: Warren Buckland (Hg.), The Film Spectator: From Sign to Mind. Amsterdam: Amsterdam University Press, S. 227-235. 


\section{Literatur}

— (1998a): (Hg.), L’Âge d'or du documentaire. Europe: Années cinquante. 2 Bd. Paris: L'Harmattan.

— (1998b): Dokumentarischer Film - dokumentarisierende Lektüre [frz. 1984]. In: Eva Hohenberger (Hg.), Bilder des Wirklichen. Texte zur Theorie des Dokumentarfims, Berlin: Vorwerk 8, S. 125-146.

— (2000): De la fiction. Bruxelles: DeBoeck.

- (2002): Kunst und Ästhetik bei Film und Fernsehen. Elemente zu einem semiopragmatischen Ansatz. In: Montage AV 11, 2, S. 42-57.

- (2003): LA FAMILLE BARTOS de Péter Forgács ou comment rendre l'histoire sensible. In: Théorème 7, S. 193-207.

— (2006): Der Eintritt des Zuschauers in die Fiktion [frz. 2000]. In: Alexander Böhnke, Rembert Hüser \& Georg Stanitzek (Hg.), Das Buch zum Vorspann. „The Title is a Shot“. Berlin: Vorwerk 8, S. 34-41.

- (2008): Reflections on the Family Home Movie as Document: A Semio-Pragmatic Approach. In: Karen L. Ishizuka \& Patricia R. Zimmermann (Hg.), Mining the Home Movie: Excavations in Histories and Memories. Berkeley: University of California Press, S. 255-271.

- (2018a): Christian Metz for Today. In: Tröhler/Kirsten, S. 91-113.

- (2018b): Das Zeitalter der Filmsprache ist angebrochen [ital. 2012]. In: Montage AV 27, 2, S. 115-128.

Roepke, Martina (2001): Feiern im Ausnahmezustand. Ein privater Film aus dem Luftschutzkeller. In: Montage AV 10, 2, S. 59-66.

Tröhler, Margrit / Kirsten, Guido (Hg.) (2018): Christian Metz and the Codes of Cinema. Semiology and Beyond. Amsterdam: Amsterdam University Press.

Wulff, Hans J. (1999): Darstellen und Mitteilen. Elemente einer Pragmasemiotik des Films. Tübingen: Narr. 



\section{Vorwort der Übersetzerinnen und Übersetzer}

Mit Kommunikationsräume erscheint erstmals eine Monografie Roger Odins in deutscher Sprache. Angesichts der breiten Rezeption, die der von Odin begründete semiopragmatische Ansatz in der deutschsprachigen Film- und Medienwissenschaft seit Beginn der 1990er Jahre erfahren hat und nach wie vor erfährt, mag dies überraschen. Während Odin seinen Ansatz in den vergangenen etwa 35 Jahren in erster Linie durch Aufsätze und Essays entwickelt und publiziert hat, trägt das hier übersetzte Buch, das im Original 2011 erschien, den Titelzusatz Einführung in die Semiopragmatik. Es bündelt Odins bisherige Schriften zur Semiopragmatik und führt in dieses Paradigma der Filmund Medienforschung ein.

Der semiopragmatische Ansatz schreibt sich in die strukturalistische und poststrukturalistische Tradition eines weiten Textbegriffs ein. Als „Texte“ gelten für Vertreter*innen dieser Perspektive (wie Claude LéviStrauss, Roland Barthes, Jacques Derrida oder Julia Kristeva) nicht nur sprachliche und schriftlich fixierte Äußerungen, sondern kulturelle Praktiken unterschiedlichster Art und in verschiedener Kodierung. Vor allem Christian Metz - für Odin eine der wichtigsten Bezugsquellen hat diese Perspektive film- und medienwissenschaftlich fruchtbar gemacht. Odins Semiopragmatik bildet vor diesem Hintergrund insofern ein Novum, als für ihn sowohl audiovisuelle Produkte (Texte) als auch institutionalisierte mediale Praktiken (Kontexte) und damit dezidiert auch der Gebrauch von und das Handeln mit Texten im Kommunikationsakt im Zentrum des Interesses stehen. In diesem Zusammenhang nimmt sein Begriff des „Kommunikationsraums“ als Bündel der Bedingungen der Möglichkeit des Funktionierens von Kommunikation in konkreten soziokulturellen und medialen Kontexten und Dispositiven eine zentrale Rolle ein.

Bei der Übertragung von Odins Text ins Deutsche sahen wir uns als Übersetzer*innen mit einigen sprachlich bedingten Problemen konfrontiert, deren Kenntnisnahme vor der Lektüre sinnvoll erscheint: So haben wir uns dafür entschieden, die Wortgruppe, die um das franzö- 
sische Wort für „Äußerung“ - énonciation - herum gebildet ist, mit den Begriffen „Enunziation“ und „Enunziator“ [énonciateur] zu übersetzen. Erstens sind die Begriffe durch vorangegangene Übersetzungen fachsprachlich etabliert; zweitens finden sich kaum vergleichbar produktive deutschsprachige Entsprechungen, die die theoretische Tiefe der auf Émile Benveniste zurückgehenden Begriffsapparatur anzeigen würden.

In manchen Fällen erforderte die Übersetzung, Kompromisse zwischen der Texttreue zur Vorlage und der Lesbarkeit im Deutschen einzugehen. Unsere zentrale Richtlinie war dabei, dem französischen Original in der Übersetzung möglichst zu entsprechen: So haben wir von Odin das generische Maskulinum ebenso übernommen wie einige Begriffe, deren deutsche Pendants zunächst ungebräuchlich wirken mögen. Ein solcher Fall zeigt sich etwa bei dem von Odin häufig verwendeten Begriff der „Relevanzachse“ [axe de pertinence], dem wir den Vorzug gegenüber der möglichen, stärker interpretativen und vom Original abweichenden Alternative der „Relevanzkriterien“ gegeben haben. Schwierigkeiten bereitete auch die wiederholte Rede von contraintes. „Zwänge“ schien uns dafür häufig zu stark, meist haben wir uns daher für das semantisch treffendere und etwas schwächer konnotierte Wort „Bedingungen“ entschieden (obwohl damit wiederum auch das Wort conditions übersetzt wurde). An einzelnen Stellen schien es uns jedoch angemessener, „Zwänge“, „Einschränkungen“ oder „Beschränkungen“ zu wählen.

Das Beibehalten des generischen Maskulinums erfolgte aus zwei Gründen: Zum einen entstehen im Deutschen grammatikalische Probleme, wenn beispielsweise „die Zuschauer*innen“ nicht mehr im Nominativ formuliert werden (beispielsweise Genitiv Singular: „der/ des Zuschauer*in/s“). Wichtiger erschienen uns jedoch theorieinhärente Setzungen: Odins Begriffe der „Aktanten“ und „Operatoren“, die an der Bildung des „Kommunikationsraums“ mitwirken und somit auch den Subjektstatus der Kommunikationsbeteiligten betreffen, sind weder empirisch fundiert noch intendiert, sondern wesentlich als heuristische Funktionsbegriffe abstrahiert und damit gerade nicht physisch-körperlich, sozial-geschlechtlich oder kulturell konkretisiert 
zu verstehen. ${ }^{1}$ Die Semiopragmatik bleibt in diesem Sinne - trotz eines damit möglicherweise unterbestimmten Subjektbegriffs - eine auf den medialen Text fokussierte Theorieperspektive; auch wenn Odin betont, dass für ihn das textbasierte „Immanentistische“ und das zuschauer*innenseitige „Pragmatische“ notwendigerweise zusammengehen müssen. Sie fokussiert in erster Linie das Produkt der Kommunikation und nicht die tatsächlich am kommunikativen Prozess Beteiligten gleichzeitig insistiert sie aber, dass Kommunikate nicht aus sich selbst heraus bereits Bedeutung enthalten, sondern die Konstruktion von Bedeutung abhängig vom kommunikativen Kontext (und der an diesem beteiligten Aktanten) entsteht.

In die Übersetzung sind hilfreiche Anregungen eingeflossen, die auf dem Workshop „Kommunikationsräume: Roger Odin und der semiopragmatische Ansatz“ des DFG-Graduiertenkollegs „Das Dokumentarische - Exzess und Entzug“ an der Ruhr-Universität Bochum im Dezember 2018 an uns herangetragen wurden. Stellvertretend für alle Teilnehmer*innen möchten wir uns hier bei dem Organisationsteam und insbesondere bei Leonie Zilch, Robert Dörre und Oliver Fahle bedanken.

Mit der Übersetzung hoffen wir, Werk und Wirken Roger Odins zu größerer Bekanntheit zu verhelfen, die Beschäftigung mit der Semiopragmatik in der deutschsprachigen Film- und Medienwissenschaft $\mathrm{zu}$ befördern und damit die wissenschaftliche Auseinandersetzung mit medialen Produkten um eine wichtige theoretische Dimension zu bereichern.

Philipp Blum, Laura Katharina Mücke, Guido Kirsten und Magali Trautmann im April 2019

1 Mit „physisch“, „sozial“ und „kulturell“ soll hier darauf aufmerksam gemacht werden, dass in Odins Konzeption auch weitere Aspekte der menschlichen Selbst- und Fremdidentifizierung wie Hautfarbe, Klassenzugehörigkeit, nationale und regionale Herkunft, Bildungshintergrund oder Migrationserfahrungen etc. nicht berücksichtigt werden. 



\section{Roger Odin}

\section{Vorwort und Danksagung}

(zur französischen Ausgabe)

Meine Überlegungen zur Semiopragmatik haben in den 1980er Jahren begonnen und sich bis heute ohne größere Unterbrechung fortgesetzt. Eine nicht geringe Zahl an Publikationen ist entstanden (von denen sich einige in der Bibliografie finden), aber was ich im vorliegenden Werk zu sagen habe, ist grundsätzlich neu. Wenn auch die Thematik früheren Veröffentlichungen entspricht, sind doch meine Herangehensweise und die eigentliche Argumentation anders geartet. Nicht nur, weil ich in einigen Punkten meine Meinung geändert habe, nicht nur, weil ich seit früheren Publikationen weitergearbeitet und daher einige Dinge präzisiert habe, sondern vor allem, weil mich die neue Reflexionsachse, die das vorliegende Buch durchzieht, dazu veranlasst hat, die Fragen in einem anderen Licht zu betrachten: Es ist das erste Mal, dass ich versuche, den Begriff des Kommunikationsraums zu theoretisieren und zu zeigen, wie er in unterschiedlichen Analyserahmen fruchtbar gemacht werden kann.

Ich habe der Idee, ein Buch über die Semiopragmatik zu schreiben, lange widerstanden. Artikel sind geschmeidiger, passen besser $\mathrm{zu}$ einem Denken, das noch auf der Suche ist. Ein Buch verfestigt das Präsentierte, wirkt kategorischer, duldet weniger Widerspruch. Auch gibt es das Risiko, prätentiös zu wirken, wenn man den eigenen Ansatz präsentiert ... Dennoch kommt irgendwann der Moment, an dem man die Notwendigkeit erkennt, wenigstens provisorisch einen Punkt zu machen, und sei es auch nur, um abzuschätzen, an welchem Punkt der Reflexion man angekommen ist (Artikel - das ist das Gegenstück zu ihrer Geschmeidigkeit - erlauben eine gewisse Unschärfe bezüglich der Kohärenz des Ganzen, ein Buch nicht). Aber ich musste mich noch entscheiden und mich an die Arbeit machen ... 
Dieses Buch wäre ohne das freundschaftliche Drängen von Bernard Miège nicht entstanden. Der Vorschlag, in einer Reihe zu erscheinen, in der es um Fragen der Kommunikation geht, wirkte sicherlich anregend. Zwar habe ich die Semiopragmatik von Anfang an so angelegt, dass sie auf alle Arten von Produktionen angewandt werden kann, aber die Texte, die ich bisher darüber geschrieben habe, bezogen sich fast ausschließlich auf den Film und andere audiovisuelle Medien. In dieser Reihe zu erscheinen hat mich angeregt, mich auch mit anderen Arbeiten zu beschäftigen. Zwar sind die Bezüge zu meinem Hauptbeschäftigungsfeld immer noch sehr präsent, aber auch andere Bereiche werden berücksichtigt. Das hat mir Vergnügen bereitet, und ich hoffe, dass die allgemeine Ausrichtung des „Modells“ deutlich wird.

Gar nicht genug bedanken kann ich mich bei den Studierenden und den Kollegen, die in diversen Seminaren, an der Universität Paris 3 Sorbonne Nouvelle, in Frankreich und in anderen Ländern, meine verschiedenen Theoretisierungsversuche ertragen haben und die mir erlaubten, durch ihre Fragen und ihre Anmerkungen, manchmal auch einfach nur, indem sie zugehört haben, immer wieder auf meine Arbeit zurückzukommen und meine Forschung voranzubringen.

Besonderer Dank gebührt Isabelle Pailliart, Bernard Miège und Pierre Mœglin für ihr genaues Lektorat. Ich habe ihren Anmerkungen stets Rechnung getragen und selbst dort, wo ich ihren Vorschlägen nicht gefolgt bin, habe ich immer auf die eine oder andere Weise davon profitiert. Es ist selten geworden, eine Reihe zu finden, die ihren Autoren ein Lektorat von solcher Qualität bietet. 
„Ohne Maschine kann man von vornherein sicher sein, nichts zu erkennen.“

Christian Metz, Essais sémiotiques (1977), S. 185 



\section{Einleitung}

\section{Das semiopragmatische „Modell“}

Ihren Ausgang nimmt die Semiopragmatik von dem erstaunlichen Befund, dass sehr viele Theoretiker größte Schwierigkeiten zu haben scheinen, sich in Bezug auf die beiden großen Paradigmen zu positionieren: das immanentistische und das pragmatische Paradigma.

Der immanentistische Ansatz sieht den Text oder die Sprache als Gegebenheit mit dauerhaften strukturellen Merkmalen an (ein System, von dem jedes Element nur in Relation zum System seinen Sinn hat), als Gegebenheit, die er ohne Bezugnahme auf alles, was dieser äußerlich ist, beschreibt. Ferdinand de Saussures Formulierung ist bekannt: „[D]ie Sprache ist ein System, das nur seine eigene Ordnung zuläßst“ (Saussure 2001 [1916], 27). Die klassische Semiologie basiert auf diesen Annahmen.

Pragmatische Ansätze nehmen umgekehrt an, dass ein Zeichen, ein Wort, eine Äußerung oder ein Text nur in Relation zu dem Kontext Sinn erhalten, in dem sie gesendet und empfangen werden. Die Theoretiker haben unterschiedliche Vorstellungen davon, was unter „in Bezug zu dem Kontext“ zu verstehen ist. Was mich angeht, so betrachte ich jene Ansätze als pragmatische, die den Kontext zum Ausgangspunkt der Sinnproduktion machen, also die Ansätze, die postulieren, dass es der Kontext ist, der diese Produktion regelt.

\section{Von der Schwierigkeit, die Immanenz zu verlassen}

Viele Versuche, das immanentistische Paradigma zu verlassen und sich dem pragmatischen anzuschließen, enden mit einer Rückkehr zum immanentistischen Paradigma. Dies ist zum Beispiel bei der Theorie der Enunziation der Fall, die ursprünglich als Analyse der zwischen einer Äußerung und dem Ensemble der Parameter der Kommunikationssituation (Sender, Empfänger und Kontext, raumzeitliche Um- 
stände, die Bedingungen der Produktion und Rezeption der Botschaft) bestehenden Beziehung, also als pragmatischer Ansatz gedacht war. Sie ist schnell zu einer Analyse des „Abdruck[s] des Äußerungsaktes innerhalb der Äußerung“ [l'empreinte du procès d'énonciation dans l'énoncé] (Todorov/Ducrot 1975 [1972], 362 [405]) reduziert worden. Oft geht diese Rückkehr zum Immanentismus mit dem Bewusstsein einher, etwas beiseite zu lassen: „Wir sind methodologisch zur Problematik der Spuren gezwungen“, schreibt etwa Catherine KerbratOrrechioni (1980, 32; Herv. R.O.).

Genauso ist es mit der „Pragmatik“ der Sprechakte (Austin 2002 [1962]; Searle 1983 [1969]), die sich darauf beschränkt zu untersuchen, wie ein Text auf seinen Leser wirkt. Der Text bleibt also an erster Stelle. Im Bereich der Filmwissenschaft wird diese Tendenz in Daniel Dayans Western graffiti (1983) deutlich. Der Autor verkündet seinen Willen, von „einer auf den Text ausgerichteten Analyse zu einer auf den Zuschauer ausgerichteten zu gelangen“ (ibid., 263) (eine Ankündigung, die sich ins pragmatische Paradigma einzuschreiben scheint), nur um sich dann der „Untersuchung der Effekte der Enunziate [énoncés]“ auf den Zuschauer zu widmen (ibid.; Herv. R.O.). Der Untertitel des Buches lautet im Übrigen: „Das Spiel der Bilder und die Programmierung des Zuschauers in Stage Coach von John Ford“. Wir bleiben im immanentistischen Paradigma.

Letztes Beispiel: der Fall von Umberto Eco, eines Theoretikers, der bereits 1962 sein Werk Das offene Kunstwerk schreibt, das mit dieser Erklärung beginnt: „Es ist eine Tatsache, dass Produktion und Konsum eines Werkes den Ausgangspunkt für zwei einander fremde Gegenstände darstellen können“ (Eco 1973 [1962], Herv. R. O.). ${ }^{1}$ Damit kann er als ein Vorreiter des textpragmatischen Ansatzes gelten. In Die Grenzen der Interpretation (Eco 1992 [1990]) allerdings widmet er eins der ersten Kapitel der „Verteidigung des wörtlichen Sinnes“ (ibid., 40) und setzt sich zum Ziel festzustellen, „was man schützen muß, um zu öffnen“ (ibid., 42; Herv.i.O.).

1 [Anm. d.Ü.:] Diese Stelle konnten wir in der deutschen Übersetzung nicht finden und haben daher nach Odins Zitat aus der französischen Ausgabe übersetzt. 


\section{Von der Schwierigkeit, in der Immanenz zu bleiben}

Man könnte meinen, dass, wenn es schwierig ist, die Immanenz zu verlassen, um sich der Pragmatik zuzuwenden, das Verbleiben in der Immanenz keine Probleme bereitet. Aber dem ist mitnichten so. Die Theoretiker, die für sich die Immanenz beanspruchen, haben offenbar ebenso große Schwierigkeiten, bei ihrem Ansatz zu bleiben, wie umgekehrt die Theoretiker, die einen pragmatischen Ansatz anstreben, ihn zu verlassen. Die Welt der Theoretiker ist wirklich kompliziert ...

Die Arbeiten des Semiologen Christian Metz illustrieren auf bemerkenswerte Weise die zweite Tendenz. Wenige Theoretiker haben so nachhaltig auf ihrer Zugehörigkeit zum immanentistischen Paradigma bestanden: die Priorität der Erforschung der Systeme; eine Methode, die auf der Untersuchung interner Differenzen basiert (Analyse von Verteilung und Komposition); der Aufbau von Typologien und Taxonomien: eine Taxonomie der sequenziellen Konstruktionen, die in Filmen vorkommen (die berühmte Große Syntagmatik; 1972 [1968]); eine Taxonomie der Sprachen; eine Taxonomie der verschiedenen „Systeme“; eine Taxonomie der „Codes“ der filmischen Sprache ...; die Liste ließe sich mühelos fortsetzen. Sein letztes Werk, Die unpersönliche Enunziation oder der Ort des Films, ist ein Manifest des Immanentismus. Metz behauptet hier nicht nur, dass Enunziator (Quelle) und Enunziatär (Ziel) „Textstücke“ sind, sondern auch, dass der „Leser im Prinzip nichts anderes [entziffert] als das, was der Schreiber hinterlegt hat [...]“ (Metz 1997 [1991], 22, 23).

Es ist ein Paradox, dass die erste Handlung von Metz bei der Begründung der Semiologie des Films darin bestanden hatte, die filmische Sprache als „Sprache [langage] ohne Sprachsystem [langue]“ (1972 [1964]) zu konstruieren und damit diesen Ansatz von vornherein in der Nähe von Modellen der Performanz und der Pragmatik zu positionieren: „Von vornherein sind wir auf Einschätzungen zurückgeworfen, die den Annehmbarkeiten (Performanz-Modelle) entsprechen und in der Rezeption die soziokulturellen Nutzer und in der Übertragung die filmischen Genres ins Spiel bringen“ $(1977,118)$. In den ersten Artikeln von Metz (1972 [1968]) sind Verweise auf die Arbeit der Sinnproduktion durch den Zuschauer sehr präsent (etwa der Begriff des „Induktionsstroms“, der suggeriert, dass der Zuschauer 
eine narrative Relation zwischen zwei Einstellungen projiziert). Sogar die Große Syntagmatik, die immer als Modell eines strukturalen immanentistischen Ansatzes genannt wird, ist so entworfen, dass sie für eine Klasse von Filmen (Spielfilme) funktioniert, die historisch beschränkt ist (klassische Spielfilme: jene, die ungefähr zwischen 1930 und 1955 produziert wurden). Auch hier bestimmen also externe (nicht-immanente) Erwägungen die Konstruktion des Modells.

Das Buch Sprache und Film (1973 [1971]) ist von pragmatischen Erwägungen scheinbar noch weiter entfernt. Aber auch hier betont Metz, dass die filmische Sprache zwar als Kombination von Codes beschrieben werden kann, aber nur das Konzept der Sub-Codes ihr tatsächliches Funktionieren $\mathrm{zu}$ einem bestimmten Zeitpunkt ihrer Geschichte erklären kann. Wenn die Codes auch das Gebiet der Probleme abstecken, die der Regisseur lösen muss (welche Kadrierung? wie die Sequenz der Bilder organisieren? wie die Anschlüsse verschiedener Bewegtbilder finden?), so gibt es doch sehr unterschiedliche Möglichkeiten, auf diese Fragen zu antworten. Die Sub-Codes sind diese „unterschiedliche[n] Antworten auf ein und dieselbe Frage“ (Metz 1973 [1971], 150). Die Frage des Filmschnitts führt zum Beispiel je nach Epochen, Autoren, ästhetischen Strömungen, Genres zu unterschiedlichen Antworten: Attraktionsmontage (Eisenstein), unsichtbarer Schnitt, Collage-Montage, „verbotener“ Schnitt (Bazin) usw. Zweifelsohne ist das Konzept des Sub-Codes ein Konzept der Pragmatik.

Allgemein ist für Metz das „linguistisch-analytische Begreifen“ von „Vornherein ein soziohistorisches Unterfangen“ (1977, 186). In „Le perçu et le nommé“ (1977, 127-161) zeigt er, dass das „Objekt der Wahrnehmung“ eine „sozial konstruierte“ Einheit ist. Der Übergang zum psychoanalytischen Ansatz (2000 [1975/1977]), der die unbewussten Determinanten in den Prozess der Filmlektüre und die Konstruktion des filmischen Signifikanten einbezieht, ist Teil derselben Denkbewegung. Metz unterstreicht schließlich, dass die von ihm geleistete Beschreibung der Funktionsweise des filmischen Signifikanten „nur bestimmte geographische Formen der Institution selbst [betrifft], nämlich solche, die in der westlichen Welt gültig sind“ und er ergänzt: „Das Kino in seiner Gesamtheit als soziale Gegebenheit und 
folglich auch der psychologische Zustand des normalen Zuschauers können Erscheinungsformen annehmen, die sich sehr stark von jenen unterscheiden, die wir gewohnt sind. Wir haben nur eine Ethnographie des Filmzustands versucht, andere sind erst zu erstellen“ (Metz 2000 [1975/1977], 108; Herv.i.O.).

Selbst in Die unpersönliche Enunziation ist der pragmatischkontextuelle Ansatz nicht abwesend. Metz zeigt darin die Modifikationen der Funktionsweise der von ihm untersuchten Konfigurationen auf, je nach dem kommunikativen Rahmen, in den sie sich einschreiben. Zum Beispiel bemerkt er, dass der Blick in die Kamera „Formen annehmen [konnte], die man gar nicht vermuten würde“; der Zuschauer des Kinos der Frühzeit betrachtete ihn als normale Sache, weil „[sich] das Dispositiv [...] entschieden von dem heute vorherrschenden unterscheidet“ (1997 [1991], 31f).

Kurz gesagt beruhen die von Metz vorgeschlagenen Analysen auf der Überzeugung, dass externe Einschränkungen die Bedingungen der Möglichkeit des Kinos bestimmen. Trotz seiner Stellungnahme für den Immanentismus kann man sagen, dass das ganze Werk von Metz von einem pragmatischen Anliegen zeugt.

\section{Ein Hin und Her}

Einige Arbeiten schließlich zeugen von einer Art Hin und Her zwischen den beiden Paradigmen. Ich beschränke mich hier auf ein Beispiel: La conversazione audiovisiva von Gianfranco Bettetini (1984) stellt Bezüge zur Konversationsanalyse her, einem der großen Zweige der linguistischen Pragmatik. Von Beginn an erklärt Bettetini jedoch, dass der Begriff der Konversation nicht dieselbe Bedeutung wie in der Linguistik haben kann: Nicht nur ist der Film ein monodirektionales Medium, das keinen wirklichen Austausch erlaubt, auch kann der filmische Text während der Konversation nicht verändert werden; es gibt also keine echte Interaktion. Andererseits spielt sich die audiovisuelle Konversation, von der hier die Rede ist, zwischen zwei Instanzen $\mathrm{ab}$, die beide über im Text vorhandene Markierungen rekonstruierbar sind: Enunziator und Enunziatär. Kurz gesagt handelt es sich um eine textuelle Konversation („,conversazione testuale“ ist der 
Titel des vierten Kapitels; 95-133). Gleichzeitig erkennt Bettetini an, dass es unerlässlich ist, sich vom Text zu lösen und die Untersuchungen auf die konkreten Situationen auszuweiten, in denen die Konversation stattfindet. Er erarbeitet auf diese Weise eine Typologie verschiedener Instanzen, die das Subjekt des empirischen Enunziators konstituieren und ins kommunikative Feld einwirken: die Linie der Herausgeber, der Autor, die großen Genres (ibid., 29). Außerdem analysiert er extratextuelle Enunziationsmarkierungen: Paratexte, Programmspalten, Genrebezeichnungen etc. (ibid., 36). Es werden also zwei Konzeptionen der Enunziation vorgestellt: eine textuelle (Enunziation im klassischen Sinne und der Immanenz entspringend) und eine externe, mit pragmatischem Status.

An anderen Stellen zeigt Bettetini, dass sich der empirische Zuschauer in die textuelle Konversation nur einschreiben kann, indem er die Stelle des Subjekts der Enunziation einnimmt, die ihm der Text anbietet, ein symbolisches Subjekt, das wie eine „Prothese“ (ibid., 28) funktioniert, auf die sich der Zuschauer stützen muss, damit die Kommunikation funktioniert. Da aber der filmische Text eine fixe, unveränderliche Struktur besitzt, die „in ihrer semiotischen Artikulation die Darstellung der Normen seiner Nutzung und der Zugangsweise zu seiner Bedeutung“ (ibid., 101) mit sich bringt, ist es am Ende der Text, der über die enunziative Prothese den empirischen Zuschauer programmiert. Wieder einmal verläuft also die Bewegung vom Text zum Zuschauer. Rückkehr zur Immanenz. Aber Bettetini erkennt auch an, dass der empirische Zuschauer sich mit der ihm zugewiesenen Position im Text nicht zufriedengeben kann, sondern zwischen zwei Vorhaben feststeckt: der Absicht des Textes und seinem eigenen Projekt, oder jedenfalls dem Projekt, das er unter den Bedingungen der äußeren Faktoren, die auf ihm lasten, bestimmen kann. Es kann also vorkommen, dass die äußeren, kontextuellen Faktoren gegenüber den textuellen die Oberhand gewinnen.

Das Buch manifestiert so ein dauerhaftes Hin und Her zwischen dem immanentistischen und dem pragmatischen Paradigma. Niemand ist sich dieser Unschlüssigkeit bewusster als Bettetini: Schon in seiner Einleitung spricht er von einer „dialektischen Spannung“ (ibid., 8) im Herzen seiner Überlegungen. Es ist offensichtlich, dass sich Bettetini 
weder von der Immanenz abwenden will, die ihm theoretische Sicherheit verspricht (laut seiner Aussage ist es die Immanenz, die die Relevanz der Semiotik sichert), noch von der pragmatischen Dimension, von der er sehr genau spürt, dass man sie nicht aussparen kann, ohne sich von der wirklichen Funktionsweise der Kommunikation zu trennen.

\section{Für eine Verbindung der beiden Paradigmen: Die Semiopragmatik}

Meiner Meinung nach sollten die Schwierigkeiten der Theoretiker, die Immanenz zu verlassen, die Position von Metz, der einen immanentistischen Ansatz beansprucht und doch einen pragmatischen entwickelt, und das Hin und Her von Bettetini nicht als inkohärent verdammt, nicht als Zeichen theoretischer Schwäche abgelehnt, sondern als Symptom eines sehr realen Phänomens verstanden werden: Und wenn man weder dem einen noch dem anderen Paradigma entkäme?

Es sieht ganz so aus, als seien die beiden Paradigmen immer schon gleichzeitig präsent gewesen, im Denken der Theoretiker aber auch im Geist jedes Einzelnen: sowohl der Glaube an den Text und seine autonome Existenz als auch das Anerkennen der Tatsache, dass sich der Sinn eines Textes mit seinem Kontext ändert.

In L'implicte (1986) beschreibt Catherine Kerbrat-Orecchioni mit Humor diese doppelte Überzeugung. Sie zitiert zunächst Bob Wilson aus einem Interview zu seiner Opernproduktion Einstein on the Beach (1976): „Ich behandele die Bilder wie ein Komponist. Man ist frei, sie so zu interpretieren, wie man mag.“ Aber auf die Frage „Was stellt dieses Gebäude dar? Eine Schule?“ antwortet er entrüstet: „Aber nein, überhaupt nicht!“. Und Kerbrat-Orecchioni kommentiert: „Diesem Widerspruch erliegen wir alle früher oder später. Als Semiotiker ist man gern bereit, das Recht auf plurale Lektüren desselben Textes einzuräumen oder sogar zu fordern und unaufhörlich zu wiederholen (da diese Wahrheit in der Welt der Kritik und unter Unterrichtenden bei weitem noch nicht mehrheitlich anerkannt wird), dass man im Herzen der interpretativen Tätigkeit ein Prinzip der Unsicherheit und der Vielfalt anerkennen muss. Sobald man aber den Semiotikerhut 
ablegt und wieder ein normaler Konsument literarischer oder anderer Texte wird, fällt man sofort wieder in den interpretativen Dogmatismus zurück, den man eben noch angeprangert hat, und rühmt hartnäckig den gesunden Menschenverstand, im Kreuzzug gegen den Widersinn: Ich weiß, aber dennoch .... (Kerbrat-Orecchioni 1986, 310).“

Jean-Daniel Lafond (1982) stellt eine umgekehrte Überlegung an, kommt aber letztlich zum selben Schluss: Er schreibt dem Zuschauer die Idee zu, dass es so viele Filme wie Lektüren gebe und dem Semiotiker die Rolle, die immanente Wahrheit des Textes wiederherzustellen: „Der Forscher [...] zentriert seine Arbeit um die Analyse von Stimuli, also um den Film als organisiertes Informationssystem. Das Vorgehen wendet sich also ab von dem Gefühl des ,Einzigartigen‘, das der Zuschauer üblicherweise bei der Vorführung eines Films hat und das das Vorurteil rechtfertigt, es gebe so viele Filme wie Zuschauer“ (ibid., 70).

$\mathrm{Ob}$ es der normale Leser oder der Theoretiker ist, der an die Variabilität des Textes glaubt, oder umgekehrt, eines ist sicher: Die doppelte Regung existiert in uns. Einerseits ist es uns unmöglich, die Existenz des Textes, also die Immanenz, nicht vorauszusetzen - ohne diese Überzeugung wäre das soziale Leben wirklich sehr schwierig. Andererseits ist es uns genauso unmöglich, nicht zu erkennen, dass die Konstruktion eines Textes je nach Kontext sehr unterschiedlich ausfallen kann (pragmatische Sichtweise).

Mir scheint die logische Schlussfolgerung besteht darin, beide Paradigmen in der Analyse der Kommunikation und im theoretischen Rahmen, der ihr Rechnung tragen soll, zu ihrem Recht kommen zu lassen. Sowenig wie sich Variabilität des Textes je nach Kontext leugnen lässt, sowenig lässt sich bestreiten, dass der Empfänger an die Existenz eines Textes mit einem unveränderlichen Sinn glaubt, den er nur dekodieren muss. Dass es sich um eine Illusion handelt, ändert nichts daran, dass es am Ende dieser Text ist, der das Resultat des Kommunikationsprozesses ist. Wir brauchen also eine Theorie, die in

2 [Anm. d. Ü.:] Mit dem Ausdruck „Ich weiß, aber dennoch ...“ [,,Je sais, mais quand même ..."] spielt die Autorin auf die psychoanalytische Theorie von Octave Mannoni an, die in den frühen 1970er Jahren einen großen Einfluss auf die Filmtheorie hatte: Mannoni, Octave (1969): Clefs pour l'Imaginaire ou l'Autre Scène. Paris: Seuil. 
der Lage ist, die beiden widersprüchlichen Bewegungen, die sich im Mittelpunkt der Kommunikation befinden, zu verbinden - eine Theorie, die die beiden Paradigmen zueinander in Beziehung setzt.

Der semiopragmatische Ansatz verkündet schon in seinem Namen die Absicht, die beiden Paradigmen zu verbinden. Die Semiopragmatik lässt den immanentistischen Ansatz der klassischen Semiologie nicht ungültig werden, deren Beitrag sie anerkennt: die Aufmerksamkeit für den Text, die Produktion von Analyseinstrumenten (eine Typologie der Zeichen, das Begriffspaar Denotation/Konnotation, paradigmatische und syntagmatische Beziehungen ...), strukturale Beschreibungen von Erzählungen und Beschreibungen, Analysen der enunziativen Strukturen, der Sprechakte ... und sogar eine Vorsicht gegenüber einem Abdriften, zu dem ein pragmatischer Ansatz, der nicht beherrscht wird, führen kann. Sein Ziel ist, den immanentistischen Ansatz in die kontextuell pragmatische Perspektive zu stellen. Wenn man die kontextuellen Bedingungen der Textkonstruktion erst einmal anerkennt, kann auch die immanentistische Analyse angewandt werden.

\section{Das semiopragmatische „Modell“}

Im Folgenden soll ein entsprechendes Kommunikationsmodell konstruiert werden. Vorsicht ist jedoch bei dem Begriff „Modell“ geboten: Ich benutze ihn aus Bequemlichkeit, aber der Begriff impliziert eigentlich eine logische Strenge, die meine Konstruktionen nicht besitzen. Die Anführungszeichen sollen auf diesen Vorbehalt hinweisen. Da es aber den Text unnötig aufblähen würde, sie bei jeder Erwähnung des Begriffs zu benutzen, bitte ich den Leser, sie mental zu ergänzen. Was ich „Modell“ nenne, ist einfach ein Arbeitswerkzeug, ein Vermittler zwischen Theorie und Beobachtung, ein theoretisches Dispositiv, eine „Maschine“ (Metz 1977, 185), eine Art optisches Instrument, eine Brille oder eher ein Mikroskop, das dazu dient, besser sehen zu helfen und sich bestimmte Fragen zu stellen.

Ich suche ein Modell, das in der Lage ist, den beiden widersprüchlichen Bewegungen Rechnung $\mathrm{zu}$ tragen: einerseits der Tatsache, dass wir das Gefühl haben, mit einem Text konfrontiert zu sein, den man uns kommunizieren wollte und den wir zu verstehen 
meinen; andererseits der Tatsache, dass verschiedene Texte produziert werden je nach dem Kontext, in dem man sich befindet. Beide Bewegungen haben nicht denselben Bezug zum pragmatischen Paradigma; wenn sich die zweite direkt darin einschreibt, entsteht die erste aus der immanentistischen Illusion, aber diese Illusion muss von der pragmatischen Position aus erklärt werden können.

Es ist klar, dass die Übertragungsmodelle vom Typ Kodierung/ Dekodierung (Shannon/Weaver 1976 [1949]; Jakobson 1971 [1960]) oder die sogenannten Y-Modelle, die sich damit begnügen, den CodeKomponenten eine pragmatische Komponente hinzuzufügen (wie etwa die deiktische Komponente bei Benveniste), zu diesem Ansatz nicht passen. Die interaktionellen Modelle von Gregory Bateson, Erving Goffman, Paul Watzlawick und anderen (vgl. Winkin 1981) sind schon näher an dem, was ich suche. Für dieses Modell ist der Kontext, der manchmal unter dem Begriff „Rahmen“, oft unter dem der „Kultur“ auftaucht, das entscheidende Element - Element in dem Sinne, in dem man von Luft oder Wasser als Elementen spricht, ein umfassendes Element also, das die Kommunikationsteilnehmer umgibt. Die entwickelte Metapher ist die eines „Orchesters“: „Die Mitglieder einer Kultur nehmen an der Kommunikation teil wie die Musiker in einem Orchester. Aber das Orchester der Kommunikation hat keinen Dirigenten und die Musiker haben keine Partition. Sie sind mehr oder weniger aufeinander eingespielt, weil sie sich gegenseitig führen“ (Winkin 2001, 90), so dass ,jedes Individuum an der Kommunikation eher teilhat als dass er ihr Ursprung oder ihr Ziel wäre“ (Winkin 1981, 25). Allerdings hat der Begriff der Kommunikation hier eine spezifische Bedeutung. Diese Modelle betreffen in der Tat die soziale Kommunikation: was sich für das Individuum und zwischen den Individuen im Hier und Jetzt abspielt. Dabei haben sie oft (wenn auch nicht immer) eine therapeutische Zielsetzung. So wie sie sind, lassen sich diese Modelle nicht übernehmen, wenn man wie ich auch die mediatisierte und verzögerte Kommunikation untersuchen will, die von einem Film, einer Fernsehsendung, einem Buch, einem Zeitungsartikel ausgeht, sowie von Zuschauern oder Lesern.

Das Modell, das ich zu konstruieren vorschlage, nimmt eine radikale Trennung zwischen dem Raum der Sendung und dem Raum der 
Rezeption an: wenn ein Sender (S) im Raum der Sendung einen Text (T) hervorbringt, so wird dieser im Raum der Rezeption auf ein Ensemble visueller und/oder akustischer Vibrationen (V) reduziert, von denen aus der Rezipient (R) einen Text produziert (T'), der a priori mit (T) nicht identisch ist. Es handelt sich also um ein Modell der NichtKommunikation.

Anmerkung: Diese Position widerspricht in keiner Weise der Behauptung von Paul Watzlawick, man könne nicht nicht kommunizieren (Watzlawick/Beavin/Jackson 2011 [1967]). Was er meint, ist, dass es unmöglich ist, keine Signale zu senden. Das heißt nicht, dass die Signale von denen, die sie wahrnehmen, auch richtig interpretiert werden. Hier eine erste Skizze der Konstruktion des Modells:

\section{[Schema 1]}

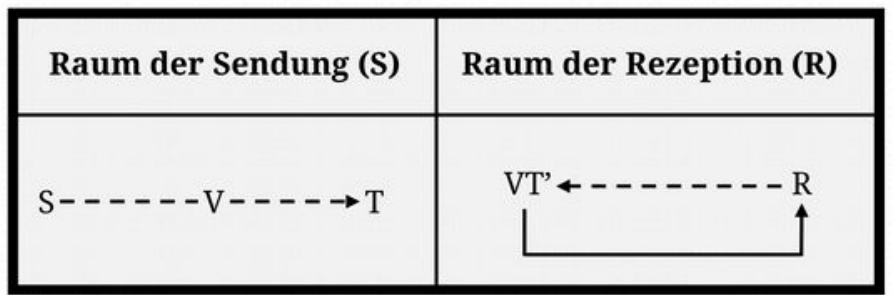

Erster Schritt in der Konstruktion eines semiopragmatischen Modells

Im Raum des Senders ist (V) zwischen (S) und (T) situiert: Die Arbeit des (S) besteht darin, Vibrationen hervorzubringen, um einen Text zu produzieren. Wenn man in den Raum der Rezeption wechselt, findet sich der Text wieder zu Vibrationen (V) reduziert und es sind diese Vibrationen, die der (R) nun seinerseits in einen Text (T') umwandelt.

Der Status, den ich diesem Modell zuschreibe, sollte unterstrichen werden: Entscheidend ist, dass es nicht versucht zu beschreiben, wie Kommunikation funktioniert. Es zielt darauf ab, sich gewisse Fragen über das Funktionieren (oder Nicht-Funktionieren) der Kommunikationsprozesse $\mathrm{zu}$ stellen. Das semiopragmatische Modell ist ein heuristisches Modell: Von einer heuristischen Hypothese fragt man nicht, ob sie wahr ist oder falsch, sondern man akzeptiert sie 
provisorisch als eine Idee, die die Forschung anleitet. Das ist der den Hypothesen der Semiopragmatik zugewiesene Status.

In den Überlegungen von vornherein von einem Modell der Nichtkommunikation auszugehen, hat einen klaren Vorteil: Aufgrund seiner Radikalität zwingt mich das Modell, die Kommunikation als Problem zu betrachten und nicht als einfach gegeben. Genauer gesagt besteht die Bedeutung eines solchen Modells darin, mich auf den Versuch zu beschränken, zu erklären was dazu führt, dass (S) und (R) „kommunizieren“; mich also zu fragen, wie die Prozesse der Sinnproduktion, die in den beiden Räumen mobilisiert werden sich dermaßen annähern, dass (R) den Eindruck hat, dass (T) von einem Raum zum anderen transportiert wurde (wie es der immanentistischen Position entspräche: (R) „glaubt“, er sei mit einem Text konfrontiert, der ihm kommuniziert wurde: Das ist die erste Bewegung).

Das Modell erlaubt auch, sich über die zweite Bewegung Gedanken zu machen, also darüber, dass verschiedene Texte (T') ausgehend von den gleichen (V) produziert werden können. Wenn nämlich (R) für die Konstruktion des Textes (T') im Raum der Lektüre verantwortlich ist, kann man sich vorstellen, dass ein anderer Rezipient (R2) einen anderen Text (T”) und ein dritter (R3) wiederum einen anderen (T’”) konstruiert, so dass es am Ende so viele Texte wie Rezipienten gibt.

Was aber verstehen wir unter einem Rezipienten und was ist, allgemeiner gefragt, der Status der Akteure der Kommunikation? Ich verstehe (S) und (R) nicht als Personen, sondern als Aktanten. Das Risiko der Abstraktion und eines gewissen Determinismus (eines rein theoretischen Determinismus allerdings) in Kauf nehmend, definiere ich (S) und (R) als Schnittpunkte des Zusammenlaufens eines Bündels von Bedingungen, die sie kreuzen und konstruieren.

Dieselbe Person kann also verschiedene Formen annehmen (R1), (R2), (Rn), je nach den Bündeln, die sie kreuzen, und sie kann daher, ausgehend von denselben (V), verschiedene Texte produzieren (T1), (T2), (Tn). Umgekehrt können aber auch verschiedene Personen die Form eines einzigen (R) haben und daher auch denselben (T) produzieren, wenn sie von demselben Bündel an Bedingungen durchkreuzt werden. 
Wenn man sich schließlich fragt, auf welche Weise die Bedingungen in jedem der beiden Räume wirken, lässt sich vergleichen wie (S) und (R), je nach dem Bündel an Bedingungen, das sie konstituiert, Sinn produzieren. Je mehr sich die Bündel ähneln, desto mehr sind (S) und (R) in analoger Weise konstruiert und desto größer stehen entsprechend die Chancen, dass sie auf gleiche Weise Sinn produzieren und sich die Konstruktionen, die sie ausführen ähneln: (T’) nähert sich so (T) an. Letzten Endes liegt es daran, dass die Aktanten der Sinnproduktion nicht frei sind, dass überhaupt Kommunikation stattfindet; genauer gesagt, es sind die Bedingungen und nur sie, die den Eindruck erlauben, dass der Kommunikationsprozess funktioniert.

Hier taucht die Frage des „Kontextes“ wieder auf, verstanden nicht als das, worauf der referentielle Vorgang verweist (wie etwa im Modell von Jakobson), sondern als das Ensemble an Bedingungen, dass die Sinnproduktion bestimmt (vgl. Schema 2 am Ende der Einleitung).

Genauso wie manche Theoretiker vorgeschlagen haben, Erzählmodelle in Modelle fertiger Erzählungen (Greimas) und Modelle, die der Bewegung der Erzählungskonstruktion durch den Leser folgen (Bremond, Eco), einzuteilen, können die Modelle der Kommunikation unterschieden werden in:

- Modelle, die die abgeschlossene Kommunikation analysieren: Modelle des Resultats (z.B. Jakobson, um die Botschaft herum zentriert); solche Modelle entstammen dem immanentistischen Paradigma;

- und Modelle, die die Kommunikation im Prozess ihres Vollzugs analysieren: Modelle der Progression (vgl. Sperber/Wilson 1986).

Das semiopragmatische Modell gehört in keine der beiden Kategorien. Es interessiert sich für die Bedingungen, die die Konstruktion der Kommunikationsaktanten regeln, und dafür, wie sie dazu angeleitet werden, Sinn zu produzieren. Ich schlage daher vor, von einem Produktionsmodell zu sprechen.

Ein Produktionsmodell beschreibt den Kontext als Konstruktion, die vor der Kommunikation kommt, um deren Modalitäten zu fixieren. Beispielsweise wird (R) im Kontext von Aufführungsorten als 
„Zuschauer“ positioniert, während (R) im schulischen Kontext als „Lernender“ oder im familiären Kontext als „Familienmitglied“ positioniert wird. In den Modellen der Progression wird der Kontext dagegen im Laufe der Kommunikation konstruiert und er fungiert dort als eine „Variable“: „Eins der zentralen Probleme der pragmatischen Theorie ist zu beschreiben, wie der Zuhörer für jede neue Äußerung einen Kontext findet, der ihm erlaubt, sie zu verstehen“ (Sperber/ Wilson 1986, 16).

Wir haben hier zwei verschiedene Konzeptionen des Begriffs Kontext, oder genauer gesagt, zwei verschiedene Kontextbegriffe. Sperber und Wilson kritisieren heftig die Modelle, die den Kontext voraussetzen. Wenn man aber die Kritik genauer ansieht, zeigt sich, dass der von ihnen kritisierte Kontextbegriff nichts mit dem der Semiopragmatik zu tun hat. So wie sie ihn verstehen, wird er vor allem von relevanten Inhalten der Enzyklopädie und von im Gedächtnis gespeicherten Informationen konstituiert (sie sprechen von „geteiltem Wissen“); für die Semiopragmatik aber besteht der Kontext aus Bedingungen. Ich glaube, dass beide Modelle sich ergänzen: Das Produktionsmodell bestimmt den Rahmen, in dem das Progressionsmodell funktioniert.

Sperber und Wilson erkennen einen solchen Rahmen im Übrigen durchaus an, aber nur für bestimmte Fälle wie etwa das Rechtswesen, wo man „tatsächlich das geteilte Wissen der verschiedenen Parteien genau festzulegen versucht: alle Gesetze und Präzedenzfälle sind öffentlich, alle Zeugenaussagen und Beweise werden aufgenommen, nur diese dürfen in Betracht gezogen werden, und so existiert ein limitierter Bereich geteilten Wissens, auf den sich die verschiedenen Parteien stützen können und innerhalb dessen sie bleiben müssen“. Aber sie ergänzen: „Nichts Vergleichbares scheint in der normalen Unterhaltung $\mathrm{zu}$ existieren und sei sie auch noch so formell und ernsthaft“ (ibid., 19). Sperber und Wilson haben natürlich recht, wenn sie den Kontext als Inhalte verstehen. Wenn man aber die Bedingungen der Sinnproduktion betrachtet, sieht es ganz anders aus. Nicht nur dass die Kommunikation sich meistens in institutionellen Rahmen abspielt, die offensichtlich Systeme von Bedingungen bedeuten (Familie, Schule, Universität, Fabrik etc.), auch Theater- und 
Kinobesuche, Fernsehgucken, ein Fußball- oder Rugbymatch im Stadion ansehen, in einen Supermarkt gehen, eine Zeitschrift aufschlagen - auch das heißt jeweils, ein Bedingungssystem zu betreten. Selbst „spontane“ Dialoge finden in derartigen Rahmen statt, wie Forscher der verbalen Interaktion und der Gesprächsanalyse gezeigt haben. Außerdem sind die Bedingungssysteme leicht $\mathrm{zu}$ transportieren und $\mathrm{zu}$ verinnerlichen: Wenn ich meinen Freund Pierre, den ich an einer Ecke treffe, nach Neuigkeiten aus seiner Familie frage, bin ich mit Bedingungen der Institution Familie und der Höflichkeit konfrontiert. Und was Liebesbeziehungen betrifft. . .

Im semiopragmatischen Modell geht die Analyse also in jedem Fall vom Kontext aus, das heißt von den Bedingungen. Diese Bedingungen führen Aktant (R) dazu, Lektüre-Hypothesen zu bilden, die er an (V) testet: Wenn ich zum Beispiel in einem Kontext bin, der mich dazu einlädt zu fiktionalisieren, werde ich versuchen, den Raum als „Welt“ zu konstruieren, es kann aber sein, dass das System der Vibrationen, auf das ich diesen Prozess anwende, eine solche Konstruktion nicht erlaubt.

Das erste Kapitel wirft die Frage nach dem Status der Bedingungen auf (natürliche, narrative, sprachliche Bedingungen etc.) und schlägt vor, den Begriff des Kommunikationsraums einzuführen, um den Aporien des Kontextbegriffs zu entkommen. Das zweite Kapitel postuliert, dass innerhalb eines Diskursraums (hier dem „westlichen“ Raum) die Akteure eine gemeinsame kommunikative Kompetenz mobilisieren. Diese Kompetenz wird verstanden als ein Reservoir an Modi der Sinn- und Affektproduktion, die sich wiederum als Kombination von Prozessen analysieren lassen. Als Beispiele werden einige Modi konstruiert. Das dritte Kapitel schlägt die detaillierte Analyse zweier Modi vor: des künstlerischen und des ästhetischen Modus, und wirft die Frage nach der Beziehung zwischen den Modi und den Kommunikationsräumen auf. Das vierte Kapitel soll anhand des Beispiels der Kommunikation des Familiengedächtnisses zeigen, wie der Begriff des Kommunikationsraums eine kontextuelle Analyse erlaubt. Das fünfte Kapitel mobilisiert den Begriff des Kommunikationsraums, um zu verstehen, was mit einer Produktion passiert, wenn sie ihren Ursprungsort verlässt. Das sechste Kapitel schließlich fragt 


\section{Einleitung}

nach dem Unterschied zwischen der traditionellen (immanentistischen) textuellen Analyse und der semiopragmatischen Perspektive.

[Schema 2]

\begin{tabular}{|c|c|}
\hline Raum der Sendung (S) & Raum der Rezeption (R) \\
\hline & \\
\hline $\begin{array}{c}\text { Kontext } \mathrm{S} \\
\text { Bedingungen } \mathrm{a}, \mathrm{b}, \mathrm{c}, \ldots \mathrm{n}\end{array}$ & $\begin{array}{c}\text { Kontext } \mathrm{R} \\
\text { Bedingungen } \mathrm{b}, \mathrm{c}, \mathrm{d}, \mathrm{g}, \ldots \mathrm{n}\end{array}$ \\
\hline
\end{tabular}




\section{Kapitel 1}

\section{Kontext, Bedingungen und Kommunikationsraum}

Wenn man den Kontext als ein Bündel von Bedingungen definiert, auf das sich das Funktionieren (oder Nicht-Funktionieren) von Kommunikation stützt, ist es notwendig, Mittel zu finden, um diese Bedingungen zu analysieren. Und hier beginnen die Probleme ..., so zahlreich sind die Bedingungen. Viele Theoretiker haben übrigens betont, dass hier auch der Stolperstein der Disziplin der Pragmatik selbst liegt. François Latraverse sieht darin ein „interessantes theoretisches und philosophisches Problem, das gelegentlich zur Verzweiflung führt“ $(1987,18)$.

Am Ende dieses Kapitels werde ich es wagen, eine Möglichkeit vorzuschlagen, sich diesem Problem anzunähern, aber zuvor möchte ich zwei Fragen stellen, die viele Diskussionen provoziert haben; zwei Fragen, von denen mir wichtig erscheint, dass sie gestellt werden, obwohl ich sie nicht wirklich beantworten kann. Die erste lässt sich so formulieren: Gibt es so etwas wie universell geltende Bedingungen für (S) und (R), die für die Aktanten den Kontext der Kommunikation bilden? Die zweite Frage bezieht sich auf die Rolle der Sprache im System der Bedingungen.

\section{Universell geltende Bedingungen}

Es sind vor allem die Kognitivisten, die diese Bedingungen betont haben. Die Kognitivisten legen Wert auf alles, was einen Menschen zum Menschen macht; darauf, dass unsere perzeptive und neuronale Veranlagung größtenteils seit Jahrtausenden die gleiche ist und heute von allen Menschen geteilt wird. Wir können daher von natürlichen Bedingungen sprechen. 


\section{Natürliche Bedingungen ${ }^{1}$}

Ein Beispiel, um die Überlegungen einzuleiten: Man kennt die These, die Umberto Eco in diversen Artikeln eingeführt und im zweiten Kapitel seines Werkes Einführung in die Semiotik wieder aufgenommen hat: „Die ikonischen Zeichen sind konventionell, was heißt, dass sie nicht die Eigenschaften der repräsentierten Sache besitzen, aber dass sie mittels eines Codes bestimmte Bedingungen der Erfahrung umschreiben“ (1972, 191). Im Gegenzug zu dieser Auffassung, die für diese Epoche der Semiologie charakteristisch ist, laden uns die Kognitivisten dazu ein, zu bedenken, dass das Wiedererkennen der Objekte im Bild durch Prozesse geschieht, die weitgehend jenen gleichen, die wir in der Welt nutzen.

Es gibt zunächst die klaren und einfachen Phänomene von Identität. Ein gezeichnetes Dreieck ist ein Dreieck, ebenso sind bestimmte typische Merkmale eines Gesichts unmittelbar in einem Bild enthalten: die mehr oder weniger runde oder ovale Form, die geometrische Anordnung, die durch die beiden Augen und den Mund gegeben ist, so wie ihre relative Position im Gesicht. Außerdem irrt Eco laut Torben Grodal (1997, 75ff), wenn er meint, dass das, was die Strichzeichnung eines Pferdes von einem in der Realität gesehenen Pferd unterscheidet, der Strich sei. Seit David Marr weiß man, dass ein Objekt zu sehen heißt, seine „virtuellen Grenzen“ zu markieren, das heißt die sichtbaren Konturen nachzuzeichnen (vgl. die Begriffe des zero crossing und des primal sketch). Ein Pferd auf einer Zeichnung wiederzuerkennen, erfordert also nicht, den Code zu beherrschen: Es reicht $\mathrm{zu}$ wissen, was ein Pferd ist. Laurent Jullier formuliert dafür eine Regel: Wenn ein bekanntes Objekt auf einer Ebene X von jemandem auf einem Bild wiedererkannt wird, ,wird dieser Jemand dadurch Besitzer des Wissens von Ebene X des Objekts“ (2002, 100).

Viele solcher Prozesse entstehen durch kognitive Automatismen, die, um zu funktionieren, weder eine Beziehung zur Kultur aufweisen, noch von einer Schlussfolgerung abhängig sein müssen: „Hat man jemals den Eindruck, einen flachen Film zu sehen? Ist es überhaupt möglich einen solchen Eindruck durch reine Willensleistung hervor-

1 Diese Passage verdanke ich in großen Teilen Jullier 2002. 
zurufen?” (Jullier 2002, 98). Versuche zeigen, dass das Gleiche für das Erkennen einer sichtbaren Bewegung, die Bestimmung der relativen Größe und den Eindruck des Reliefs gilt. Dieser Typ von Prozess betrifft selbst die Effekte, die nicht durch die Produktion von Bedeutung ausgelöst werden, wie das Empfinden von Schwindel, die Veränderung des Drucks und der Geschwindigkeit, die man in manchen Simulationsmaschinen oder durch IMAX-Filme fühlen kann: unmöglich, dem zu entkommen. Um diese Ebene zu charakterisieren, sprechen Kognitivisten von einem Kurzschluss, von Vorverkabelung (der Begriff beschreibt die neuronalen Verbindungen, die bei oder schon vor der Geburt existieren), von der Ebene „subsymbolischer Verbindungen“ und von verkapselten Modulen: Module, die nicht bewusst vom Subjekt kontrollierbar sind und die man daher nicht einfach abschalten kann.

Es scheint jedoch angebracht, einen Punkt zu betonen: Um valide $\mathrm{zu}$ sein, muss diese Vorgehensweise einem äußerst kontrollierten Experimentierprozess folgen. Oft wird dieser Punkt jedoch von denjenigen, die auf kognitivistischer Grundlage zur Kommunikation arbeiten, vernachlässigt: Sie selbst machen kaum Versuche und vertrauen auf die Ergebnisse von Experimenten aus anderen, sehr technischen und hoch spezialisierten Disziplinen (Neuroanatomie, Neurophysiologie, Neurobiologie, Künstliche Intelligenz, Kognitionspsychologie ...). Da sich die Experten untereinander nicht einig sind und es diverse Strömungen (Beispiel: Computationismus vs. Konnektionismus) und oftmals gegenläufige Vorschläge gibt, ist es für den Kommunikationsforscher schwierig, sich auszukennen und vor allem zwischen diesen Vorschlägen auszuwählen, weil er nicht die Kompetenz besitzt, sie einzuschätzen.

Umgekehrt nehmen sich die Wissenschaftler bei ihren Versuchen oft nicht genug vor den Fallen der Kommunikation in Acht. Das in dieser Hinsicht größte Risiko besteht darin, dass die Forscher den Versuchen ihre eigene Art $\mathrm{zu}$ kommunizieren und ihr eigenes Weltverständnis zugrunde legen (das ist die naturalistische Illusion). So haben schon viele Theoretiker geglaubt beweisen zu können, dass die tonale Musik auf natürlichen Prinzipien der menschlichen Kognition beruht, und so auf die Existenz einer natürlichen und impliziten 
auditiven Grammatik geschlossen, die zum Beispiel die Schwierigkeiten erkläre, die das Publikum beim Hören von serieller Musik empfände: Man sei nicht darauf programmiert, Serien zu hören. Die Kompositionsgrammatik, die von Komponisten dieser Art von Musik genutzt wurde, stünde demnach im Konflikt mit der natürlichen Grammatik der Rezeption (Lerdahl/Jackendorff 1983). Und dennoch hat man genauso viele Schwierigkeiten, das Spiel von musikalischem Thema und Gegenthema in den Fugen von Bach zu erkennen, wie die Serien der seriellen Musik. Und wie lässt sich außerdem erklären, folgt man dieser Theorie, dass die Polyphonie der Pygmäen für die Pygmäen selbst ohne Probleme funktioniert, während wir unfähig sind, ihre Rhythmen korrekt zu erkennen (Gribenski 2005)? Zeigt dies nicht, dass es musikalische Grammatiken gibt (hergestellte und gehörte Grammatiken), die anders sind als unsere, und die demnach die Idee unplausibel erscheinen lassen, die tonale Grammatik sei das Ergebnis einer natürlichen Bedingung? Damit soll nicht gesagt sein, dass es so etwas wie Universalität im Bereich von Musik gar nicht gäbe - schon der Begriff der Musik (im Vergleich zu Gesprochenem oder zu Lärm) scheint ein universelles Phänomen $\mathrm{zu}$ beschreiben, ebenso wie bestimmte Prinzipien der musikalischen Konstruktion (Spannung vs. Entspannung; Wiederholung vs. Nicht-Wiederholung) - aber mit ihrer theoretischen Verortung muss man vorsichtig sein.

Mit Jullier lässt sich noch anfügen: Je mehr man sich von der Ebene automatischer Bedingungen entfernt, um sich symbolischen Typologien zuzuwenden, desto mehr entfernt man sich von Prozessen, die für empirische, indiskutable Experimente Raum lassen, wodurch Modellierungen umso hypothetischer werden. Wenn die Forschung zum Gedächtnis auf einer noch (mehr oder weniger) verifizierbaren Modellbildung beruht, wird die Gültigkeit aller Modellbildungen, die den menschlichen Geist betreffen (manchmal auch als Theorie des Geistes bezeichnet), im Sinne eines durch den Computer inspirierten Informationsverarbeitungssystems, von der Beantwortung folgender Frage abhängen: Sind beide Systeme gleicher Natur? Die Debatte ist eröffnet. Das Problem ist noch brisanter bei allem, was dem evolutionistischen Ansatz angehört. Wie lässt sich beweisen, dass meine Begeisterung für eine bestimmte Landschaft, die ich mit meiner 
Gefährtin teile, daher rührt, dass diese schon von unseren Vorfahren aus dem Pleistozän geschätzt wurde (vgl. die „savanna hypothesis“ von Orians/Heerwagen 1992)?

Die narrative Bedingung

Es gibt allerdings eine universelle Bedingung, die scheinbar indiskutabel ist: die narrative Bedingung. Theoretiker verschiedenster Disziplinen sind sich einig: Es existiert eine innere Kraft, die uns in Richtung der narrativen Strukturen treibt. Ethnologen bestätigen: Die Erzählung wird in allen Gesellschaften verwendet. Psychoanalytiker sprechen von der „Wirkung eines dem Seelenleben innewohnenden Wunsches“ (Baudry 2003 [1975], 50), ein Wunsch, der sich direkt aus dem ödipalen Zwang ergibt: „Heißt erzählen denn nicht immer, nach seinem Ursprung $\mathrm{zu}$ forschen, seine Händel mit dem Gesetz zu benennen, in die Dialektik von Liebe und Hass einzutreten?“ (Barthes 1974 [1973], 70). Psychologen legen dar, dass die „mise en intrigue“ unsere Erfahrungen von unserer frühen Kindheit an strukturiert. Dieser Bezug zu dem von Ricœur geformten Begriff ist nicht zufällig: Was hier verhandelt wird, ist die Frage nach der „narrativen Identität“. Der Jugendpsychiater Daniel Stern (1998 [1995]) beschreibt am Säugling etwas, das sich ,vorläufige Gefühlsform‘ nennt (temporary feeling shape), wie eine Kombination von zeitlich strukturierten Affekten im Modus Spannung-Krise-Auflösung, welche die fundamentale narrative Spannungslinie erzeugt. Diese Form verbindet das Schema des Affekts mit dem der Narration. Oder wie es der klinische Psychologe Denis Mellier formuliert: „Stern stellt Narrativität als eine wahrhaftige Erfahrung frühzeitiger, infraverbaler Symbolisierung dar“ (2003, 2). Mellier merkt auch an, dass diese Idee bereits unterschwellig in der Beschreibung des „Fort-Da“ bei Freud angelegt ist. In neueren Texten spricht Stern von „affektiver Narration“ und vom „narrativen Modell“, eine Konstruktion, die im dritten Lebensjahr zustande kommt, ein „Modell, das zum Einsatz kommt, wenn man sich selbst oder jemand anderem etwas erzählt“ (1990, 30-35). Der Neurobiologe Antonio Damasio geht noch weiter und spricht vom „narrativen Funktionieren des Gehirns“: „Geschichten erzählen im 
Sinne des Erfassens dessen, was im zerebralen System ankommt, das ist wahrscheinlich eine Obsession des Gehirns, die früh beginnt und zeitgleich im Verlauf der Evolution und der Ausbildung der Komplexität neuronaler Strukturen erforderlich wird, um Erzählungen zu schaffen. Geschichten erzählen kommt vor dem Sprechen, es ist sogar eine Bedingung für die Sprache ...“(zit. n. Bellour 2009, 182f).

Die Verinnerlichung der narrativen Bedingungen hat für die Kommunikation wichtige Konsequenzen. Kein Bereich der Kommunikation entgeht der Narration. Die Narration durchdringt Literatur, Comic, Theater, Ballett, Presseartikel, Küchenrezepte, Lieder, Wetterberichte, Nachrichten (im Radio oder Fernsehen), quasi alle Fernsehprogramme (auch das Reality TV ist gescriptet). Im Bereich des Kinos sind die nicht-narrativen Filme äußerst selten, ebenso im experimentellen Kino. Auch Dokumentarfilme haben immer öfter eine narrative Form. Die Entwicklung des Storytellings in der Presse, in der Unternehmenskommunikation oder der Politik ist ohne Zweifel die wichtigste und reizvollste der letzten Zeit. Die Narration hält ebenso Einzug ins alltägliche Leben: Unterhaltungen, die wir führen, sind meistens nur eine Aneinanderreihung von Mikronarrativen; Spiele, die Kinder erfinden, aber auch Spiele auf digitalen Konsolen, sind offensichtlich narrativ. Tatsächlich nehmen wir die Welt durch diese narrativen Strukturen wahr und regeln mit ihnen unsere Handlungen. Eben diese Strukturen versucht eine Grammatik der Erzählung, wie jene von Claude Bremond (1973), zu erfassen. Sie beschreibt die Erzählung als Interrelation von Rollen im Verlauf einer Handlung und ihr höchstes Ziel besteht in der Ausarbeitung einer universellen Theorie menschlichen Handelns.

Die Kehrseite der Medaille ist, dass Formen, die nicht narrativ sind, weniger gut funktionieren. Die diskursive Form hat sicherlich ihre Berechtigung, aber jeder weiß, dass ein Diskurs besser funktioniert, wenn er Erzählstrukturen verwendet; sei es durch Minierzählungen im Inneren des Diskurses selbst, sei es durch eine vollständige Umwandlung in eine Erzählung, wie es die Fabel, die Parabel, die propagandistische Erzählung, der moralisierende Roman, der Lehrroman und eine große Anzahl von Filmen (vor allem US-amerikanische Spielfilme) machen. Im französischen Fernsehen hat Alain 
Decaux mit seinem Talent als Geschichtenerzähler die Geschichte ins öffentliche Bewusstsein gebracht. Die poetische Form erreicht dagegen nur wenige Leser. Die Beschreibung wiederum ist der ungeliebte Teil der Lektüre: Wer kann sich schon damit rühmen, noch nie eine Beschreibung übersprungen zu haben?

Eine einzige Struktur zieht sich geschickt aus der Affäre: die Liste. Listen aus wissenschaftlichen Absichten, Listen aus praktischen Absichten (etwa Inventare aller Art, Kommissionslisten, To-do-Listen), „poetische“ Listen. In Die unendliche Liste (2009 [2009]) zeigt Eco, dass es eine Reihe von Autoren gibt, die Listen lieben: Homer, Rabelais, Cervantes, Calvino, Prévert, Borges, Perec ... und Eco selbst. Der Anthropologe Jack Goody nennt jedoch zwei wichtige Einschränkungen: Auch wenn es eine wissenschaftliche Befriedigung im mündlichen Zitieren von Listen gibt (Gefallen am Spiel des Klangs, Gefallen am Rhythmus, der mehr oder weniger obsessive Gefallen an Aufzählungen), sind - abgesehen vom Aufzählen von Vorfahren, dem Rezitieren des Stammbaums, den Listen von Heiligen, den Litaneien in der Religion und manchen spielerischen Situationen - „die Gelegenheiten für mündliche Auflistungen extrem selten“ (1977, 108). Außerdem ist die Liste eine Struktur, die geschrieben besser funktioniert als gesprochen. Grundsätzlich hat die Liste nicht die gleiche strukturierende Kraft wie die Narration: Was die Struktur betrifft, so öffnet sich die Liste ins Unendliche (weil man immer noch einen Punkt hinzufügen kann), während sich jede Narration von ihrem Ende her konstruiert. Hinzu kommt, dass die Liste eine gewisse Kohärenz hervorrufen kann, aber nur aufgrund der Kopräsenz ihrer Elemente, während die Narration durch Kausalketten funktioniert. Experten für Mnemotechniken raten dazu, Listen zu narrativieren, um sie einfacher erinnern zu können (einigen kognitiven Anthropologen zufolge speichert das menschliche Gehirn Informationen besser, wenn sie in Kausalketten vorliegen). Die Liste ist also weit davon entfernt, einen so prägnanten Status zu haben wie die narrative Form.

Der Druck der narrativen Form ist übrigens so stark, dass mitunter auch nicht-narrative Strukturen narrativ gelesen werden. Während einer Reihe von Experimenten im Bereich des Films machte Paul S. Cowen eine interessante Entdeckung: „Es schien mir sinnvoll anzu- 
nehmen, dass Filme mit nicht-narrativen Strukturen und besonders solche, deren narrative Strukturen eindeutig dysfunktional sind, den Betrachter aus dem ideologischen Gefängnis befreien würden, in das narrative Filme ihn unmerklich einschließen ...“; doch er nimmt die Annahme schnell zurück: „Die Betrachter schienen nicht den Wunsch zu verspüren, ihr narratives Schema zu verlassen - trotz der Inkohärenz einiger Szenen oder des ganzen Films“ (2002, 46). Er gesteht: „Ich habe nicht erwartet, dass die Tendenz, das narrative Schema zu nutzen, um visuellen Erfahrungen Kohärenz zu verleihen, dermaßen stark ist“" (ibid., 48).

Ich fasse zusammen: Wenn man die Existenz universeller Bedingungen akzeptiert (und es scheint schwierig, dies nicht zu tun), muss man anerkennen, dass die Produktion von Bedeutungen und Affekten auf einer bestimmten Ebene immer auf die gleiche Art im Raum der Sendung und in dem der Rezeption ausgeführt wird, was die Abtrennung beider Räume schwächt, ohne sie verschwinden zu lassen: Eine Vielzahl an Ebenen ist von diesen notwendigen Prozessen nicht betroffen. Aus theoretischer Sicht macht es Sinn, das Modell in zwei Räume aufzuteilen, denn dies zwingt den Theoretiker dazu, Beweise für die Existenz geteilter Bedingungen zu finden: Man vermeidet es, die Universalität dieser oder jener einfachen Prozedur vorauszusetzen, nur weil man sich selbst an sie gewöhnt hat.

Man könnte auch meinen, dass die Existenz solcher Prozesse eine der Grundannahmen des semiopragmatischen Modells in Frage stellt, nämlich die, dass das, was uns übermittelt wird, lediglich Vibrationen sind (V). Und es ist wahr, dass es wegen dieser notwendigen Prozesse üblicherweise keine Vibrationen sind, die wir verspüren: „Selbst beim lebenslangen Couch-Potato lernt das visuelle System nicht irgendwann, den Bildschirm als eine Scheibe strahlender Phosphor-punkte zu erkennen“ (Pinker 2009 [1999], 29). Aber auch hier kann die modellhafte Annahme einer Übertragung von Vibrationen dazu führen, sich zu fragen, auf welcher Ebene und auf welche Weise die notwendigen Prozesse die Vibrationen in Bedeutungen und Affekte verwandeln.

Letztlich können jedenfalls dadurch, dass man universelle Bedingungen herausstellt, „den anderen Diskursen Grenzen aufgezeigt 
werden“: Es ist unnütz, nach weiteren Bedingungen zu suchen (in der Kultur, der Gesellschaft ...), wenn diese ohnehin universell programmiert sind; unnütz, sich Fragen über eine Wahl zu stellen, wenn man keine Wahl hat (Jullier 2002, 198).

\section{Der Fall der Sprache}

Anscheinend gibt es nichts Universelles am Gebrauch von Sprache. Im Gegenteil, man beißt sich an ihrer Pluralität die Zähne aus. Dennoch finden sich Theoretiker, und nicht die schlechtesten, welche die Existenz einer universellen Grammatik behaupten oder zumindest linguistische Universalien hinter der Diversität der Sprachen vermuten. Bekanntlich war dies die Position der Grammaire générale et raisonnée von Port-Royal (Arnauld \& Lancelot 1999 [1666]). In jüngerer Vergangenheit hat Noam Chomsky (1973 [1965]; 1971 [1966]) die Hypothese von gemeinsamen Strukturen hinter allen Sprachen aufgestellt, von Strukturen, die dem menschlichen Geist inhärent sind. Für ihn kann nur die Existenz einer universellen, uns inhärenten Syntax die Schnelligkeit erklären, mit der Kinder Sprache lernen. Diese Schnelligkeit schließe die Hypothese aus, dass Lernen über „trial and error" funktioniert. Es scheint als wären wir prädisponiert, eine Grammatik zu lernen, als wäre diese Kenntnis bereits in die Struktur der Sprachfähigkeit eingeschrieben. Die universelle Grammatik konstruiert also eine positive Bedingung (Chomsky beschreibt seine universelle Grammatik als eine „unbewusste Bedingung“). Dieser Hypothese wird jedoch heute von diversen Kognitivisten widersprochen (z.B. Deacon 1997; Liberman 1998).

Man kann auch die Sprache an sich als eine Bedingung ansehen (oder als ein Ensemble von Bedingungen). Wir suchen uns unsere Muttersprache nicht aus: Durch unsere Geburt in eine Gemeinschaft werden wir dieser oder jener Sprache zugewiesen (die Sprache [langue] „ist ein Schatz, den die Praxis des Sprechens in den Personen, die der gleichen Sprachgemeinschaft angehören, niedergelegt hat“, Saussure 2001 [1916], 16). Die Sprache ist sogar eine Voraussetzung für alle Bedingungen, die an den Körper gebunden sind: Man wird in eine 
Sprache hineingeboren, die vor uns da war und in die unser Leib eingeschrieben wird.

Dass die linguistische Kommunikation durch unsere Beziehung zur Sprache bedingt ist, erleben wir jedes Mal, wenn wir uns einem Gesprächspartner aus einem anderen Land gegenübersehen. Interessanter ist es allerdings zu untersuchen, welche Rolle die Sprache außerhalb des linguistischen Kommunikationsraums spielen (oder nicht spielen) kann.

Sprache und Wahrnehmung

Die berühmte „Sapir-Whorf-Hypothese“ (Whorf 1963 [1956]) besagt, dass Sprache die fundamentalsten Kategorien unseres Denkens bestimmt; - die Kategorien Zeit, Raum, Subjekt und Objekt sind auf Englisch zum Beispiel nicht dieselben wie in der Sprache der HopiIndianer - genau wie unsere Art, die Welt in Objekte aufzuteilen. Das Beispiel, das immer zitiert wird, ist das der Inuits und des Schnees. Da ihre Sprache ein Dutzend Worte kennt, um Schnee zu bezeichnen, existieren für sie zehn unterschiedliche Schnee-Objekte, während ein Franzose nur ein Schnee-Objekt sieht, dem er eventuell differenzierende Eigenschaften zuschreiben kann: kristallisierter Schnee, geschmolzener Schnee, vereister Schnee, Pulverschnee ... (Schaff 1965). Ein anderes Beispiel findet sich in Forschungen zum Zahlensystem in diversen Sprachkulturen des Amazonas: Es scheint festzustehen, dass eine Verbindung zwischen dem lexikalen Zahlenreichtum einer Sprache und der Fähigkeit ihrer Sprecher besteht, bestimmte arithmetische Operationen durchzuführen. Die Sprache beeinflusst also die kognitiven Kapazitäten (Pica/Lemer/Yzar/Dehaene 2004; Gordon 2004).

Allerdings ist die Hypothese von Sapir-Whorf auch scharf kritisiert worden: einerseits von Ethnologen, die Zweifel an den Bedingungen dieser Experimente angemeldet haben, andererseits von Kognitivisten, die der Meinung sind, dass die Repräsentation der Welt sich durch eine interne und universelle Sprache formt, die Sprache des Denkens - die Mentalese, eingeführt von Jerry Fodor (1975) -, und dass die Gliederung durch Sprachen nur ein Oberflächeneffekt ist, der unsere Wahrnehmung von der Welt an sich nicht beeinflusst. 
Das einzige, was man angesichts dieser Diskussionen sagen kann, ist, dass die Debatte hierüber noch nicht abgeschlossen ist.

Der Einfluss der Sprache auf die Lektüre von Bildern

Auf einer ersten Ebene ist die Frage nach dem Einfluss der Sprache auf die Lektüre von Bildern an das eben genannte Problem gebunden: Wenn man die Objekte der Welt der Sprache entsprechend aufteilt, wird diese Aufteilung auch für die Objekte eines Bildes funktionieren.

Aber man muss auch an die Hypothese von Michel Colin erinnern, die besagt, dass auch auf der Ebene der Vektorisierung der Sprache eine einschränkende Bedingung existiert, nicht nur auf der Ebene der Semantik. In Langue, Film, Discours (1985) legt Colin nahe, dass man in der westlichen Zivilisation die Lektüre (und die Produktion) von Bildern wie den schriftlichen Diskurs vektorisiert, also von links nach rechts. Bestimmte anthropologische Arbeiten scheinen in die Richtung dieser Hypothese zu gehen. In The Domestication of the Savage Mind (1977) zeigt Jack Goody, wie Tabellen und Listen eine Rolle in der Konstruktion bestimmter Denkformen spielen, und dass man nicht mehr auf die gleiche Art denkt, sobald es eine geschriebene Sprache gibt. Goody erwähnt beiläufig die Frage nach der Linearität des geschriebenen Textes und die nach ihrer Orientierung (horizontal oder von oben nach unten) und unterstreicht, dass dies „entscheidende Konsequenzen hat für die Art, wie Informationen eintreffen (input) und besonders dafür, wie sie herausgehen (output) und ebenso für den Empfänger“ (1977, 124). Für ihn ist das Wesentliche, dass man mit dem Geschriebenen den Bedingungen der zeitlichen Abfolge ausweichen kann: Die Schrift ermöglicht es, ans Ende zurückzukehren, von einer Passage zur nächsten zu springen ... Aber er sagt nichts über die Vektorisierung (des Signifikats), von der Colin spricht, denn sein Vorschlag betrifft die Gegenüberstellung von Schriftlichem und Gesprochenem und nicht die Struktur von dieser oder jener Sprache. Falls allerdings bestimmte grafische Figuren fähig sind, wie Goody sagt, „das Innenleben der Psyche“ zu modifizieren (ibid., 108), warum sollte dann die Vektorisierung der linguistischen Signifikanten nicht auch das Lesen und die Produktion von Bildern beeinflussen können? 
Wenn man Bilder unter diesem Gesichtspunkt betrachtet, entdeckt man eine Vielzahl an bewegten und unbewegten Bildern, die so konstruiert scheinen, als hätte man, willentlich oder nicht (dies könnte auf eine implizite Grammatik verweisen), diese Vektorisierung berücksichtigt.

Was die audiovisuelle Sprache betrifft, erwähnt Colin besonders die Bedeutung von Schwenks: Für einen Zuschauer, dessen Sprache eine Vektorisation von links nach rechts aufweist, erscheint ein Kameraschwenk von links nach rechts nichts Außergewöhnliches, während im Gegenzug der Schwenk von rechts nach links auffällig ist und einen starken Effekt produziert. Etwa einen Effekt der Präsentation: „Und hier kommen die, die man erwartet“, sagt uns der Schwenk von links nach rechts, der PARTIE DE CAMPAGNE (EINE LANDPARTIE, Jean Renoir, F 1936/1946) eröffnet, und der uns die Familie Dufour zeigt, die am Rande des Flusses in einem Pferdewagen ankommt (ich habe diesen Film detailliert analysiert in Odin 2000). Oder einen Effekt der Destabilisierung: Am Anfang von ÉTAT DE SIÈGE (DER UNSICHTBARE AufsTAND, F/I/BRD 1972), nutzt Costa Gavras mehrere Schwenks von rechts nach links entlang der Autoschlangen, die durch die nach Terroristen fahndenden Polizeikontrollen verursacht wurden (hervorragende Vorbereitung des Zuschauers auf den Fortgang des Films).

Colin erwähnt ebenfalls, dass neue Elemente häufig in einer Plansequenz durch Schwenks oder seitliche Fahrten von links nach rechts eingeführt werden. Jullier (2002, 80) kritisiert diese Analyse, indem er darauf aufmerksam macht, dass im Gegensatz dazu die berühmte Plansequenz, die Touch of EVIL (IM ZEICHEN DES BöSEN, Orson Welles, USA 1958) eröffnet, durch eine Bewegung von rechts nach links gekennzeichnet ist. Doch diese Plansequenz könnte umgekehrt als ein exzellentes Beispiel der Bestätigung der Links-Rechts-Vektorisierung gelesen werden: Die Kamerabewegung von rechts nach links wird benötigt, um den Zuschauer zu destabilisieren und ihn emotional in den Zustand vor der Explosion des in die Falle gelockten Autos am Ende der Sequenz zu versetzen. Es reicht nicht aus festzustellen, dass es im klassisch-narrativen Film viele Plansequenzen gibt, die sich von rechts nach links bewegen. Das beweist gar nichts, denn es ist möglich, dass diese Plansequenzen von rechts nach links genau darauf aus sind, 
einen bestimmten Effekt beim Zuschauer zu erzielen. Man muss also analysieren - und ich muss sagen, dass die Analysen, die ich gemacht habe, eher in Richtung der Hypothese von Colin weisen.

Colin hat die Konsequenzen seiner Hypothese für die Kommunikation deutlich gemacht: „Wenn diese Hypothese, die Bedeutung der Lektüre des filmischen Bildes - und noch allgemeiner: des figürlichen Bildes - betreffend, bestätigt wird, folgt daraus, dass der Vorgang der Figuration, und besonders die Produktion filmischer Diskurse, als ein Prozess gesehen werden muss, der einer gegebenen linguistischen Tradition folgt - der Tradition der westlichen Linguistik.“ Und er fügt hinzu: „Es ist nicht [...] ausgeschlossen, dass sich diese Orientierung von links nach rechts auch in anderen Kinematografien findet, die diese linguistische Tradition in ihrer Schriftsprache nicht kennen (zum Beispiel im arabischen Film); dies würde beweisen, dass das westliche Kino in seiner Eigenschaft als dominantes Kino nicht nur seine semantischen Modelle, sondern auch seine syntaktischen Strukturen durchsetzt“ (1985, 104). In den Ländern, deren Sprache nicht auf der Links-Rechts-Vektorisierung beruht, kann man sagen, dass das Kino wie eine zweite Sprache, gesprochen' wird, egal ob man Regisseur oder Zuschauer ist. Die Regisseure aus diesen Ländern lernen ihr Handwerk übrigens oft im westlichen Kino oder von westlichen Professoren; was die Zuschauer betrifft: Wenn sie Filme verstehen wollen, sind sie dazu gezwungen, die Vektorisierung zu lernen, die diesen zugrunde liegt ein Lernprozess der sich implizit vollzieht. Wenn man dieser letzten Bemerkung von Colin folgt, gäbe es also eine Form des kulturellen Kolonialismus auf der Ebene der kinematografischen Sprache selbst.

\section{Die „nicht-natürlichen“ Bedingungen und die Idee des „Kommunikationsraums“}

Ich weiß nicht, ob die Hypothese von Colin stimmt. Jedenfalls kann darin eine Aufforderung gesehen werden, den an die Sprache gebundenen Bedingungen in dem Modell einen gewissen Stellenwert zuzuschreiben, und sei es nur, um diese Frage überhaupt im Blick zu behalten (ich glaube, dass die Kognitivisten den Einfluss der Sprache unterschätzen). Außerdem zwingt uns diese Frage dazu, eine Reihe 
anderer Bedingungen $\mathrm{zu}$ berücksichtigen, die ökonomischen und politischen Bedingungen, denn wenn die westliche kinematografische Sprache als dominant betrachtet wird, heißt das, dass der Westen die Macht gehabt hat, seine kinematografische Sprache anderen Teilen der Welt aufzuzwingen.

Es sind die Cultural Studies, die vor allem an diesen „nichtnatürlichen Bedingungen“ interessiert sind, die die Kommunikation beeinflussen. Ihr Zugang verfährt genau gegenteilig $\mathrm{zu}$ dem der Kognitivisten. Statt sich der Frage zuzuwenden, ob es eine Universalität des Menschlichen gibt, betonen diese Forscher die Differenzen, die Alterität, die Idiosynkrasien sowie die Diversität der Gemeinschaften und die Pluralität von Identitäten. Der Austausch zwischen diesen beiden Lagern ist alles andere als friedlich, aber ich sehe nicht, warum die Cultural Studies und vor allem die Berücksichtigung von Kulturen und sozialen Bräuchen den kognitivistischen Ansatz verhindern sollten und umgekehrt. Einige Kognitivisten, die offener sind als andere, teilen übrigens diese Ansicht (Jullier zum Beispiel).

Das Problem mit dieser Art Zugang ist, dass wenn man beginnt, die kontextuellen Bedingungen aufzulisten, die die Kommunikation regeln, man kaum mehr damit aufhören kann. Janet Staiger zeigt, dass der filmische Text der kreativen Aneignung durch die Zuschauer ausgeliefert ist - Zuschauer, die selbst durch den Kontext bestimmt sind, in dem sie sich entwickeln (,es gibt keine freien Leser“; Staiger 2000, 162). Wie man sich in Bezug auf einen Film verhält, erörtert Staiger, indem sie eine Liste von Bedingungen aufzählt: Alter, „Rasse“, Ethnie, biologisches Geschlecht, sexuelle Präferenz, nationale Identität, Beruf. Zu FerRis Bueller's DAY Off (FERrIS MACHT BlaU, John Hughes, USA 1986) bemerkt sie, dass die Reaktionen auf den Film unterschiedlich seien, je nachdem, ob der Zuschauer ein englischsprachig aufgewachsener, männlicher, heterosexueller Jugendlicher sei, der dem Mittelstand oder der Oberschicht angehört, eine junge Lesbe, ein Homosexueller, jemand mit hispanischen Wurzeln, ein junger Mensch aus der Arbeiterklasse, oder ob man es mit jemandem zu tun hat, der die kapitalistische Ideologie bzw. die Konsumgesellschaft und die Dominanz des männlichen Geschlechts akzeptiert 
oder nicht. Es gibt noch weitere Bedingungen, die Staiger erwähnt: Bedingungen, die an die Erziehung geknüpft sind: das Respektieren von Stille im Konzertsaal hängt mit der Herausbildung eines bürgerlichen Publikums zusammen; ibid., 44-51; Bedingungen, die an einen professionellen Habitus gebunden sind: Wissenschaftler schätzen das Kognitive vor dem Affektiven und vor allem gehen sie von einem kooperativen, kohärenten Zuschauer aus, der gewillt ist, die Absicht des Films zu verstehen, und sich nicht nur ablenken und amüsieren will (vgl. ibid., 38f); intertextuelle Bedingungen: sich nach den Gründen fragend, warum sie lachen musste, als sie THE TEXAS ChaINSAW MASSACRE (Tobe Hooper, USA 1974) sah, glaubt Staiger, dass dies daher rührt, dass sie den Film in Beziehung zu Psycho (Alfred Hitchcock, USA 1960) und dem „morbiden Gefühl für Humor“ von Hitchcock gesetzt hat (ibid., 179-187); psychoanalytische Bedin-gungen: für Janet Staiger beeinflussen auch diese Bedingungen ihre Reaktion auf The Texas ChainsaW Massacre: „Wie Freud mit Bezug auf den Witz notiert, sind die Ökonomien der aufgewandten Energie an die Hemmungen gebunden“ (ibid., 185). Und es wäre nicht schwer, Staigers Liste um weitere Bedingungen zu ergänzen.

Man könnte angesichts dieser ausufernden Kontexte verzweifeln. Janet Staiger ist sich dieses Problems übrigens durchaus bewusst: „Reaktionen von Subjekten empirisch zu untersuchen, ist ein kompliziertes Unterfangen, voller theoretischer und historischer Probleme“, und sie präzisiert, dass eine dieser Schwierigkeiten insbesondere darin besteht, festzulegen, „welche Identitäten durch die Interaktion zwischen einem Individuum und einem spezifischen Text entstehen" (ibid., 115-124). Um es in meinen eigenen Worten zu sagen: zu definieren, wie die Aktanten der Kommunikation durch die kontextuellen Bedingungen konstruiert werden.

Um diese Aporien zu vermeiden, schlage ich vor, vom Begriff des „Kontexts“ zu dem des Kommunikationsraums überzugehen. Der Begriff des Kommunikationsraums stellt dabei den Versuch dar, den Kontext zu „modellieren“.

Definition: Ein Kommunikationsraum ist ein Raum, der durch ein Bündel an Bedingungen bestimmt ist, das die Aktanten (E) und (R) 
dazu treibt, Bedeutung entlang derselben Achse der Relevanz zu produzieren. $^{2}$

Die Idee ist nicht neu: Kommunikationsexperten haben verstanden, dass sie, wenn sie eine Chance erhalten wollen, gehört zu werden, versuchen müssen, den Unterschied zwischen dem Raum der Emission und dem Raum der Rezeption zu reduzieren, indem sie derartige Räume konstruieren, um ihre Empfänger in sie einzuschreiben: Die Modellierung zielt dann darauf ab, den Kontext zu gestalten. Ich berücksichtige zwei solcher Verfahren, aber es gibt ohne Zweifel weitere.

Das erste Verfahren besteht darin, ein System aus externen Bedingungen zu konstruieren, aufgrund derer die Empfänger so kommunizieren, wie man es wünscht. In der Massenkommunikation sind es die Genres, die dazu dienen, die Erwartungshaltung der Empfänger zu regeln, indem sie die Achse der Relevanz festlegen, die für die Lektüre angelegt wird. Raphaëlle Moine spricht von „Genreschienen“ (2002, 80). Die Programmstruktur des Fernsehens erfüllt dieselbe Funktion. Und auch die Mehrheit der großen Institutionen (Schulen, Kirchen ...) wenden solche Konstruktionen an.

Das zweite Verfahren schreibt die Bedingungen in die Nachricht selbst ein, darauf hoffend, dass die Empfänger die Achse der Relevanz erkennen, von denen der Sender hofft, sie mögen angelegt werden: Das ist die Methode des segmented audience, die darin besteht, nur ein spezifisches Publikum anzusprechen (französische Teenager, die „fünfzigjährige Hausfrau“, junge homosexuelle Paare ...).

Aus semiopragmatischer Perspektive unterscheidet sich der Begriff des Kommunikationsraums von dem Begriff des Kontextes in etwa so wie sich die Begriffe langue und langage in der Theorie von Ferdinand de Saussure unterscheiden. ,Kontext‘ ist ein unscharfer Begriff, der ein heterogenes, wenn nicht sogar bunt gemischtes Ensemble an Bedingungen abdeckt (ein Ensemble, das analytisch nicht zu bewältigen ist); ein Kommunikationsraum ist eine Konstruktion, die von Theoretikern

2 [Anm. d.Ü.:] Der Begriff „Achse der Relevanz“ (auf Französisch: „axe de pertinence“) ist etwas sperrig. Gemeint sind in vielen Fällen die Kriterien, die bei der Untersuchung angelegt werden und die regeln, was wichtig und was unwichtig ist. Wir haben uns für einen Ausdruck entschieden, der möglichst nah am Original ist. 
vorgenommen wird. Ein Kommunikationsraum hat daher nichts Konkretes an sich. Er ähnelt in dieser Hinsicht dem Begriff des „mentalen Raums“, wie ihn Gilles Fauconnier (1984) geprägt hat, aber, abgesehen davon, dass sich seine Funktionsweise auf größere Einheiten als Sätze bezieht, hat er nicht den kognitiven Status, den ihm dieser Autor zuschreibt. Hier ist es der Theoretiker, der die Achse der Relevanz bestimmt und der für die Konsistenz des Kommunikationsraums sorgt, zu dem er arbeiten oder den er als Analysewerkzeug nutzen möchte.

Leser, die mit früheren Arbeiten von mir vertraut sind, haben vielleicht bemerkt, dass ich hier den Begriff des Kommunikationsraums anstelle des Begriffs der Institution verwende: Mir ist klar geworden, dass der Begriff der Institution zu sehr Gefahr läuft, als Struktur verstanden $\mathrm{zu}$ werden, die in der Wirklichkeit existiert. Außerdem kann ein Kommunikationsraum etwas ganz anderes sein als eine Institution in der üblichen Wortbedeutung. Allerdings erachte ich die Definitionen von Institutionen, die ich gegeben habe, insofern weiterhin als gültig, als sie den Akzent auf einen weiteren Punkt legen, der mir immer noch wichtig erscheint, nämlich den Begriff der Sanktion. So habe ich Cornelius Castoriadis zitiert: „Die Institution ist ein symbolisches, gesellschaftlich sanktioniertes Netz“ (1984 [1975], 226). Und Alain Berrendonner: Die Institution ist „eine normative Macht, die Individuen wechselseitig unter Androhung von Sanktionen bestimmten Praktiken unterwirft“ (1981, 95). Wenn die Aktanten der Kommunikation die Bedingungen des Kommunikationsraums, in dem sie sich befinden, nicht respektieren, bezahlen sie dafür auf die eine oder andere Weise. Es macht Sinn, sich nach der Art der Sanktionen zu fragen, die in diesem oder jenem Kommunikationsraum gelten: Im Fernsehen kann das die Langeweile sein, die ich bei einem sentimentalen Film empfinde, wenn ich einen Thriller erwartet habe; in der Schule eine im Mitteilungsheft vom Lehrer eingetragene schlechte Note; in der zwischenmenschlichen Kommunikation ein Konflikt zwischen Familienmitgliedern etc. 
Folgende Prinzipien regeln die Konstruktion eines Kommunikationsraums:

a) Der Theoretiker hat das Recht, dem Kommunikationsraum, mit dem er arbeiten will, den genauen Grad und die Art der Allgemeinheit oder Besonderheit (also genau die Extension) zuzuschreiben, die er sich bezogen auf Objekt, Raum und Zeit wünscht. Er kann zum Beispiel entscheiden, über den pädagogischen Kommunikationsraum (Objekt) im Allgemeinen zu arbeiten (die Parameter von Zeit und Raum sind hier nicht berücksichtigt), oder auch über den pädagogischen Kommunikationsraum in der Schule der Dritten Republik (Zeit) in Frankreich (Raum), oder über den pädagogischen Kommunikationsraum im Lauf der Geschichte (Objekt), heute (Zeit), in Frankreich (Raum) etc.

b) Der Theoretiker hat das Recht (und es besteht die Notwendigkeit), diesen Raum auf eine begrenzte Anzahl von kontrollierbaren Parametern zu reduzieren. Dies ist das Prinzip der Relevanz: Der Kommunikationsraum ist das Resultat einer Auswahl an Bedingungen, die den Prozess der Bedeutungsproduktion regulieren und entlang der Achse der Relevanz funktionieren, die der Theoretiker für seine Analyse ausgewählt hat. Wenn ich zum Beispiel entscheide, zum pädagogischen Kommunikationsraum im Allgemeinen $\mathrm{zu}$ arbeiten, beziehe ich mich nur auf jene Bedingungen, die diesen vom zwischenmenschlichen und alltäglichen Kommunikationsraum unterscheiden, vom spielerischen Kommunikationsraum, von dem der Nachrichten usw. Ich beziehe mich dagegen nicht auf historische, kulturelle oder ideologische Unterschiede pädagogischer Konzepte.

c) Wichtig ist, diese Beschränkungen herauszustellen. Dies ist eine der Lehren, die ich von Christian Metz behalten habe: „Es reicht aus, es zu sagen“ war einer seiner bevorzugten Ratschläge. 


\section{[Schema 3]}

\begin{tabular}{|c|c|}
\hline Raum der Sendung (S) & Raum der Rezeption (R) \\
\hline S- & $\mid$ \\
\hline \multicolumn{2}{|c|}{ Allgemeine Bedingungen } \\
\hline \multicolumn{2}{|c|}{ Sprachliche Bedingungen } \\
\hline \multicolumn{2}{|c|}{ Bedingungen des „Kommunikationsraums“ } \\
\hline
\end{tabular}





\section{Kapitel 2}

\section{Der diskursive Raum Kommunikative Kompetenz und Modi der Sinnproduktion}

Ich nehme hypothetisch an, dass man für einen gegebenen historischen Zeitpunkt einen sehr großen Kommunikationsraum konstruieren kann, in dem die Akteure homologe Prozesse der Sinnproduktion mobilisieren. Das Ensemble dieser Prozesse bezeichne ich als diskursive Kommunikationskompetenz. Die diskursive Kommunikationskompetenz ist das Reservoir, aus dem sich die Teilnehmer (Sender und Empfänger) bedienen, um Sinn zu produzieren, indem sie diesen oder jenen Prozess aktivieren.

So wie er hier verwendet wird, sollte der Begriff der Kompetenz nicht mit dem gleichnamigen Konzept verwechselt werden, das Noam Chomsky (1971 [1966]; 1973 [1965]) auf dem Gebiet der Linguistik erarbeitet hat. Laut Chomsky ist die linguistische Kompetenz angeboren, genetisch als Potenzial angelegt, und daher von jedem Einfluss des Kontexts unabhängig. Auch ist sie auf die Syntax beschränkt, d.h. auf die Fähigkeit, Sätze zu bilden. Die Kompetenz, wie ich sie hier verstehe, ist dagegen auf einen Kommunikationsraum bezogen und hat eine viel größere Ausrichtung als jene des Satzbaus: Es handelt sich um eine diskursive Kompetenz der Kommunikation. Mit dem Gebrauch dieses Begriffs stelle ich mich weniger in die Tradition von Jürgen Habermas als in die von Dell Hymes (1974) und jenen Forschern, die Hymes folgten (in Frankreich etwa Sophie Moiran und Patrick Charaudeau).

Die diskursive Kommunikationskompetenz ist nur eine Komponente der kommunikativen Kompetenz. Die Zahl und der jeweilige Status der Komponenten der kommunikativen Kompetenz variieren von Forscher zu Forscher, aber abgesehen von der Tatsache, dass sich 
alle (trotz terminologischer Unterschiede) über die Existenz einer diskursiven Komponente einig sind, lassen sich, wenn man entsprechende Gruppierungen vornimmt, noch drei weitere Komponenten festhalten: eine semio-linguistische Kompetenz, eine soziokulturelle Kompetenz und eine referenzielle Kompetenz.

Die semio-linguistische Kompetenz betrifft das Beherrschen der Sprachen: der natürlichen (geschriebenen und gesprochenen) Sprachen, der gestischen Sprache, der Bildsprache, der filmischen Sprache etc. Im semiopragmatischen Modell hängen an dieser Kompetenz die Ausführungen der Prozesse in der jeweiligen Sprache.

Die soziokulturelle Kompetenz betrifft die interaktive und soziale Dimension der Kommunikation. Im semiopragmatischen Modell betrifft sie sowohl das Verhältnis der Akteure zum Kommunikationsraum als auch das Verhältnis der verschiedenen Akteure zueinander.

In Bezug auf die referenzielle Kompetenz, die das Verhältnis zur Erfahrung und den Dingen der Welt und damit unmittelbar den produzierten Sinn betrifft, und ihren Platz im semiopragmatischen Modell, lässt sich nicht viel sagen, da der produzierte Sinn immer soziohistorisch ist. Daher können ihn nur empirische Analysen im genau (historisch, geografisch, soziologisch) situierten Kommunikationsraum begreifen. Das semiopragmatische Modell selbst bezieht sich nicht auf Inhalte, sondern auf Prozesse. Die Inhalte werden erst in den ausgeführten Fallstudien wieder reintegriert.

Eine Bemerkung zur Terminologie: Aufgrund seiner Besonderheit, besonders bezüglich seines großen Umfangs, werde ich im Folgenden von diskursivem Raum sprechen und damit den Raum bezeichnen, der die diskursive Kommunikationskompetenz regelt. Den Ausdruck Kommunikationsraum werde ich auf die auf einer darunterliegenden Ebene konstruierten Räume beschränken (pädagogischer, wissenschaftlicher, künstlerischer, familiärer etc. Kommunikationsraum), also auf Räume, die als im diskursiven Raum inbegriffen verstanden werden: Diese Räume wählen die Prozesse der diskursiven Kommunikationskompetenz aus, die von den Aktanten der Kommunikation mobilisiert werden.

Auch die Frage nach dem Bezugsfeld des diskursiven Raums muss gestellt werden. Trotz des unscharfen, schwer zu fassenden, ja sogar 
widersprüchlichen Charakters des Begriffs „westlich“ - er transportiert, wie Claude Prudhomme im Artikel „Occident“ im Dictionnaire des concepts nomades en sciences humaines treffend formuliert, eine „Ideologie, die die heutige Epoche kompromittiert“ $(2010,356)$ - glaube ich, dass der im vorliegenden Buch konstruierte diskursive Raum dem westlichen Kulturraum angehört. So wie ich das Adjektiv verwende opponiert es gegen seine ideologische und noch mehr gegen seine polemische Verwendung. Für mich ist die Rede von dem „westlichen“ Raum zunächst einmal eine Vorsichtsmaßnnahme: um anzuzeigen, dass die beschriebenen Prozesse in einem begrenzten Raum funktionieren (sofern sie funktionieren). Das Adjektiv „westlich“ soll der Tatsache Rechnung tragen, dass die Prozesse ausgehend von meiner Erfahrung als Akteur der Kommunikation konstruiert werden sowie von meinem Eindruck, dass diese Erfahrung von einer großen Zahl anderer Akteure geteilt wird. So benutze ich „westlich“ mangels besserer Alternativen laut Prudhomme gibt es keine „Lösung der Ersetzung“ (ibid., 343) weil es das Wort ist, das mir spontan in den Sinn kommt, wenn ich an die Gemeinschaft von Akteuren denke, der ich mich zugehörig fühle, aber es sagt nichts über die geografische oder historische Eingrenzung dieser Akteure aus. Es ist durchaus wahrscheinlich, dass einige der Prozesse bei Akteuren, die nicht aus dem „Westen“ im üblichen Wortsinn (Europa und das englischsprachige Amerika) stammen, auch funktionieren. Ich glaube auch nicht, dass eine Definition der genauen Ausdehnung dieses Raums möglich ist. Ich teile mit Alexandre Zinoviev (1995) (der, um Missverständnissen zu entgehen, von „Okzidentismus“ spricht) die Idee, dass es notwendig ist zu abstrahieren: So wie ich den „westlichen“ Raum verstehe, ist er lediglich eine theoretische Fiktion. Auch ist es wichtig darauf hinzuweisen, dass diese Konstruktion weder Werte (der Westen wird oft anhand von Werten definiert: Freiheit, Christentum, Humanismus, wissenschaftliches Denken ...) noch Inhalte (geografischer, historischer, kultureller, zivilisatorischer Art ...) betrifft, sondern Prozesse sowie, allgemein gesprochen, Phänomene, die der sozialen Anthropologie (oder Sozialanthropologie) angehören.

In diesem sowie im folgenden Kapitel wird es vor allem um Modi gehen, das heißt um bestimmte Kombinationen von Prozessen. Als Modi bezeichne ich theoretische Konstruktionen, die darauf abzielen, 
die Prozesse der Sinnproduktion in funktionale Ensembles zu strukturieren. Die Konstruktion der Modi antwortet auf drei Fragen:

1. Welche diskursiven Formen setzt dieser Modus um: Narration, Beschreibung, „Diskurs“ (im engeren Sinn einer argumentativen Konstruktion), ${ }^{1}$ poetische Struktur etc.?

2. Welche affektiven Beziehungen erzeugt er?

3. Welche enunziativen Beziehungen, welche(n) Enunziator(en) lädt er zu konstruieren ein?

Es kann manchmal sinnvoll sein, zur ersten Frage eine Ergänzung anzubringen: 1a. Welche Art von Raum wird durch den Modus konstruiert? Eine Welt, ein Ensemble von Zeichen, ein symbolischer oder „diskursiver“ Raum, ein abstrakter Raum, ein plastischer Raum etc.? Zusammen gefasst ergeben diese Fragen eine Fragestellung, nämlich: Zu welchem Typ kommunikativer Erfahrung führt der Modus?

Das Hauptinteresse der genannten Fragen ist analytischer Art: Indem man sie systematisch stellt (wenn auch nicht unbedingt in dieser Reihenfolge, je nach Modus kann es praktischer sein mit einer anderen zu beginnen) und indem man ein System von Gegenüberstellungen ins Spiel bringt, kommt man zur Konstruktion einer Reihe verschiedener Modi. Es ist hier nicht mein Ziel, eine Liste dieser Modi zu präsentieren, sondern anhand einiger Beispiele die Probleme vorzuführen, die sich für den Theoretiker ergeben.

Um die Konstruktion der Modi anschaulicher zu machen, werde ich sie ausgehend von Produktionen darstellen, die für den jeweiligen Modus exemplarisch sind (d.h. die erzeugt wurden, um in diesem oder jenem Modus gelesen zu werden). Es ist allerdings stets zu bedenken, dass diese Produktionen nur in dem Maße exemplarisch sind, als ich davon ausgehe, dass die Aktanten der Kommunikation bereit sind, das Spiel des Kommunikationsraums mitzuspielen, für das die Werke vorgesehen sind. Die Produktionen könnten in einem anderen Kommunikationsraum auch ganz anders gelesen werden. Und umgekehrt

1 Ich verwende „Diskurs“ und „diskursiv“ in Anführungszeichen, wenn ich jene spezifische argumentative Diskursform meine. 
kann jeder Modus auch außerhalb seines eigentlichen Kommunikationsraums aktiviert werden. Ich gebe dafür später einige Beispiele.

\section{Der fiktionalisierende Modus (erste Annäherung)}

Wenn es eine Erfahrung gibt, die von allen geteilt wird, dann ist dies die Erfahrung der Fiktion. Es erscheint daher nicht absurd, die Konstruktion der Modi mit dem Versuch zu beginnen, einen Modus zu konstruieren, der dieser Erfahrung Rechnung tragen kann: dem fiktionalisierenden Modus. Man könnte meinen, dass die sehr starke Präsenz dieses Modus dessen Konstruktion vereinfachen müsste, er also leicht zu identifizieren wäre, aber dem ist nicht so.

Die erste Schwierigkeit für den Theoretiker besteht schon in dem Wort „Fiktion“ selbst. Es gibt nämlich die Tendenz, es für alles Mögliche zu verwenden. Selbst bei Theoretikern ist diese Tendenz vorhanden. So behauptet Metz: „Jeder Film ist ein fiktionaler Film“ (2000 [1975/1977], 45). Und Éric Clemens meint: „Die gesamte Literatur [...] ist [...] fiktional, selbst die realistische, selbst Memoiren und Autobiografien“ (1993, 276, Fn20). Aus Sicht der beiden Forscher mögen diese Aussagen eine gewisse Berechtigung haben, aber in der Perspektive einer Kommunikationsanalyse (besonders einer Analyse, die sich auf die Erfahrungsdimension konzentriert), können sie nur kontraproduktiv sein. Wenn alles Fiktion ist ...

Die Vermischung von Fiktion und Narration, wie sie von anderen Autoren betrieben wird, ist nicht weniger problematisch. Wie Laurent Gerverau (2000, 399) anzunehmen, dass eine "gefilmte Reportage immer eine Fiktion ist“, weil es sich dabei um die „Montage einer Narration durch eine oder mehrere Person(en)“ handelt, erlaubt es nicht, den Unterschieden der kommunikativen Erfahrung Rechnung zu tragen, die zwischen dem Lesen oder Sehen einer Reportage und dem Lesen oder Sehen einer Fiktion bestehen. Außerdem würde, wenn die Aktivierung einer narrativen Konstruktion gleichbedeutend mit der Fiktionalisierung wäre, wiederum fast alles zur Fiktion. Wie oben gesehen, nutzen wir die Narration in fast allen kommunikativen Situationen. Es ist daher notwendig, bei der Charakterisierung dieses Modus restriktiver vorzugehen (Odin 2000). 
Ich schlage vor, den fiktionalisierenden Modus durch eine Serie von Prozessen zu charakterisieren.

a) Auf der Ebene des Raums: Konstruktion einer Welt, d. h. eines Raums, der zumindest potenziell bewohnbar ist, und sei dies nur durch den Rezipienten (Diegetisierung).

Ich lese Emile Zolas Der Bauch von Paris (1873): „Inmitten der tiefen Stille zogen durch die menschenleere, ansteigende Allee die Karren der Gemüsegärtner nach Paris mit dem gleichmäßigen Kreischen ihrer Räder, dessen Widerhall an die Mauern der Häuser schlug, die zu beiden Seiten der Straße hinter den verschwommenen Linien der Ulmen in nächtlicher Ruhe dalagen. “2 Von den ersten Zeilen an vergesse ich die schwarzen Flecken auf der Seite, die Wörter, vergesse ich die Syntax des Satzes (das ist der Vorteil von arbiträren Konstruktionen: hat man einmal die Regeln gelernt, vollzieht sich der Zugang zum Sinn unmittelbar) und befinde mich selbst auf der Allee zwischen den Karren und den Gemüsegärtnern. Fiktionalisieren, das heißt zunächst, in eine Welt einzutreten.

b) Auf der diskursiven Ebene: Konstruktion einer Erzählung (Narration).

„Die Blicke auf das Lichtmeer von Paris gerichtet, dachte Florent über die Geschichte nach, die er verheimlichte. Nachdem er aus Cayenne geflohen war ...“ Einige Worte reichen, um meine Imagination als Leser in Bewegung zu setzen, um mich wissen wollen zu lassen, was es mit der Figur Florent und seiner Geschichte auf sich hat, kurz gesagt, um mich auf eine narrative Konstruktion einzulassen; ich brenne darauf, zu erfahren, wie es weitergeht ...

2 Zit.n.der Übers.v.Armin Schwarz: https://gutenberg.spiegel.de/buch/der-bauch-von-paris1251/1; zuletzt aufgerufen am: 17.07.2019. 
c) Auf der Ebene der affektiven Beziehung: eine derartige Konstruktion des Textes, dass ich im Rhythmus der erzählten Ereignisse mitschwinge. Dies ist der Prozess der Mise en phase.

Beispiel: Zola unterbricht die Enthüllungen des Fräuleins Saget über Florent (,er kommt aus dem Zuchthaus ..."), um über vier Seiten verschiedene Käsesorten zu beschreiben. So steigert er bei uns die Erwartung, genauso wie sie bei den Frauen steigt, die auf die Erzählung warten, welche von dem Klatschweib mit diebischer Freude hinausgezögert wird ... Die Mise en phase passt die affektive Positionierung des Rezipienten (hier des Lesers) den Verhältnissen innerhalb der Diegese an.

d) Auf der enunziativen Ebene: Konstruktion eines Enunziators, dem ich keine Fragen bezüglich Identität, Tun und Wahrheit stellen darf. Dies nenne ich den Prozess der Fiktivisierung der Enunziation.

Sobald ich mir Fragen über die Identität des Enunziators stelle (wer hat den Text geschrieben? wer war Zola?) oder über die Weise, wie der Text konstruiert ist (wenn ich beispielsweise beginne Zolas Kunstfertigkeit in den Beschreibungen zu bewundern, seine Weise mit den Metaphern zu spielen, wie mit der des Meeres, die die genannte Passage durchzieht) oder über die Wahrheit des Geäußerten (stimmt es, dass die Rue du Pont Neuf bei der Seine beginnt und zur Rue Montmartre und zur Rue Montorgueil führt?), dann verlasse ich die fiktionalisierende Lektüre. Fiktionalisieren, das heißt an die Existenz einer Welt zu „glauben“, ohne sich Fragen über ihren Ursprung zu stellen. Umgekehrt sind alle Fragen erlaubt, die sich auf Ereignisse in dieser Welt beziehen: Warum wurde Florent ins Zuchthaus geschickt? Wie ist er entkommen? Was hat er in Paris vor?

Ich spreche von einem realen Enunziator, wenn man eine Äußerungsinstanz konstruiert, der man eine Identität, ein Tun und Werte zuschreibt und deren Äußerungen man einen Wahrheitswert zumisst, und von einem fiktiven Enunziator, wenn man die Instanz als 
nicht hinterfragbar konstruiert, wie im Fall des fiktionalisierenden Modus.

Diese Weise, den Enunziator zu konstruieren unterscheidet die Fiktionalisierung radikal von der Lüge, von der Hypothese oder vom Irrtum, die alle einen realen Enunziator voraussetzen, den man hinsichtlich der Wahrheit befragen kann.

Schließlich, und das ist wohl das wichtigste Phänomen vom Standpunkt der Kommunikation betrachtet, führt die Fiktivisierung des Enunziators auch zur Fiktivisierung des Rezipienten: Indem ich akzeptiere, mir keine Fragen über den Enunziator zu stellen, situiere ich mich gewissermaßen außerhalb der realen Welt.

Definition des fiktionalisierenden Modus (erste Annäherung):

- auf der räumlichen Ebene: Konstruktion einer Welt (einer Diegese);

- auf der diskursiven Ebene: Konstruktion einer Erzählung;

- auf der affektiven Ebene: Beziehung der Mise en phase zu den erzählten Ereignissen;

- auf der enunziativen Ebene: Konstruktion eines fiktiven Enunziators.

\section{Spektakularisierender Modus, energetischer Modus}

Im Vergleich zur üblichen Verwendungsweise des Begriffs der Fiktion, ist die Art, in der ich den fiktionalisierenden Modus konstruiert habe, stark vereinfachend. Viele Produktionen, die allgemein als „Fiktionen“ bezeichnet werden, finden sich außerhalb der Fiktionalisierung; sie erzeugen nicht die Erfahrung der Fiktion, wie ich sie gerade beschrieben habe. Um ihrer kommunikativen Funktionsweise gerecht zu werden, bedarf es also der Konstruktion anderer Modi.

In Schriften zum Kino werden Musicals der Fiktion zugerechnet; ihre Erfahrung entspricht jedoch nur teilweise dem fiktionalisierenden Modus. Bei Tanz- und Gesangsnummern werden zwei Verschiebungen gegenüber der Fiktionalisierung vollzogen: die Verwandlung der „Welt“ in einen „Raum des Spektakels“ und der Übergang von einem fiktiven zu einem realen Enunziator. 
Ich schaue Singin' IN THE RAIN (Stanley Donen \& Gene Kelly, USA 1952). Don Lockwood und sein Freund Cosmo Brown unterhalten sich, während sie durch die Studios Monumental Pictures spazieren, wo gerade die Arbeiten in vollem Gange sind. Don Lockwood ist traurig, da er immer noch an das junge Mädchen denkt, in das er verliebt ist. Als er zu einem Klavier kommt, beginnt Cosmo, um seinen Freund aufzuheitern, mit einer atemberaubenden Nummer, in der sich Gesang, Tanz und Akrobatik mischen. Am Anfang erleben wir Cosmos Possen aus Sicht von Don Lockwood, an den sie sich in der Diegese richten und der sich auf einem Stuhl im Bildvordergrund niedergelassen hat, um seinem Freund zuzusehen. Im Fortgang der Sequenz autonomisiert sich Cosmos Nummer dann mehr und mehr. Der Sound, der bis dahin diegetisch war (Cosmo spielt Klavier), wird durch eine Music-over abgelöst, zu der Cosmo tanzt. Bald verschwindet Don Lockwood aus dem Bild; man sieht ihn nicht einmal am Ende der Sequenz wieder, wie man vielleicht erwarten könnte. Vielmehr endet sie mit einer Überblende zur nächsten Sequenz. Wir vergessen also völlig die Anwesenheit von Don Lockwood und interessieren uns nur noch für die Extravaganzen von Cosmo. Er rollt über den Boden, läuft an Wänden hoch, springt durchs Dekor und singt und tanzt dabei im Rhythmus der Musik.

Selbst wenn sich der Raum anscheinend nicht verändert hat (wir sind immer noch im selben Studio, die Bühnenarbeiter tragen weiter Dinge durchs Bild, die Cosmo zum Teil für seine Gags nutzt), hat sich meine Beziehung zum Repräsentierten doch grundlegend verändert. Während mich der Anfang der Sequenz noch dazu einlud, in die Welt der erzählten Geschichte einzutreten und das Studio in Begleitung der beiden Freunde zu durchqueren, bin ich danach auf meine Position als „Zuschauer“ zurückgeworfen. Zwischen der Welt, in der Cosmo seine Nummer aufführt, und der meinen hat sich eine unsichtbare Barriere errichtet. Die Welt der erzählten Geschichte macht Platz für einen Raum des Spektakels. Gleichzeitig werde ich dazu gebracht, die Performance des Pianisten, Sängers, Tänzers und Akrobaten zu bewundern, der Cosmo Brown spielt. Ich konstruiere also nicht länger einen fiktiven Enunziator - die Figur verschwindet -, sondern, im Gegenteil, einen realen: Donald O'Connor, also einen Enunziator, den ich bezüg- 
lich seiner Identität und seins Tuns befragen kann, was bei der enunziativen Konstruktion der fiktionalisierenden Lektüre nicht möglich wäre.

In solchen Momenten verlasse ich die Fiktionalisierung zugunsten des spektakularisierenden Modus.

\section{Definition des spektakularisierenden Modus:}

- räumliche Ebene: Erzeugung eines Raums des Spektakels, das heißt eines Raums, der vom Raum des Zuschauers durch eine sichtbare (Orchestergraben, Theatervorhang, Leinwand im Kinosaal) oder unsichtbare Barriere (in unserem Kopf) getrennt ist;

- diskursive Ebene: selbst wenn es eine Neigung zur Narration gibt, kann der spektakularisierende Modus jeden diskursiven Prozess aktivieren;

- affektive Ebene: die Beziehung baut sich nicht zur Figur auf wie in der Fiktionalisierung, sondern zu den realen Personen, den Sängern, Tänzern, Darstellern etc.;

- enunziative Ebene: Konstruktion eines realen Enunziators, der hinsichtlich seiner Identität und seines Tuns befragbar ist.

Auf diesen Modus greife ich angesichts von Liveaufführungen oder Fernsehaufzeichnungen derartiger Spektakel zurück: Theaterstücke, Konzerte, Ballett, Variété, Zirkus, Boxkampf, Fußballspiele oder Rugby etc. Auch Tier- und Naturfilme funktionieren oft in diesem Modus (man spricht auch von „Naturspektakeln“). Aber auch im Alltag passiert es mir, dass ich diesen Modus anwende, wenn ich beispielsweise Urlauber am Strand beobachte oder einen politischen Amtsträger, der bei einer Veranstaltung das Wort ergreift ...

Es gibt noch ein weiteres Ensemble an Produktionen, die üblicherweise der Fiktion zugerechnet werden, die aber die Fiktionalisierung zum Teil blockieren: Produktionen, die im energetischen Modus funktionieren.

Der erste Bruch mit der Fiktionalisierung vollzieht sich auf der diskursiven Ebene: In manchen Momenten von NATURAL BORN KILLERS (Oliver Stone, USA 1994) destrukturiert die hyperschnelle Montage komplett die Narration; bei James Cameron (TERMINATOR, 1994; ALIENS, 
1986) sind es die Blinkeffekte, die Blitzlichter und Stroboskop-Effekte, die dieselbe Wirkung haben. In solchen Sequenzen wird die Erzählung weniger wichtig als der Rhythmus. Selbst bei eindeutig narrativen Filmen kann es dem vom visuellen und akustischen Fluss erfassten Zuschauer passieren, sich für eine gewisse Zeit von der Erzählung zu lösen (zum Beispiel bei der STAR WARS-Serie, George Lucas, USA 19772005).

Der zweite Bruch, der mit dem ersten zusammenhängt, betrifft die affektive Beziehung. In diesen Momenten geht es nicht mehr darum, im Rhythmus der erzählten Ereignisse mitzuschwingen (Mise en phase), sondern im Rhythmus der Bilder und Töne. Die Affekte weichen den Effekten. Um derartige Produktionen $\mathrm{zu}$ charakterisieren hat Richard Corliss das Wort Dyna-Movies geprägt (Time v. 2. Juli 1990, S. 46): Filme, die mehr dynamisch als dramatisch sind. Laurent Jullier (1997) spricht von „Orchester-Filmen“ und „Feuerwerk-Kino“, um damit Filme zu bezeichnen, die für kurze Zeit den Intellekt des Zuschauers ausschalten, um direkt den sensorischen Apparat zu berühren.

Das ist eine der Besonderheiten dieses Modus: Zwar gibt es immer noch eine Sinnproduktion, aber diese wird auf den zweiten Platz verwiesen, das Wichtige spielt sich im Verhältnis zum Körper ab. Es geht nicht um Ideen oder eine Geschichte, sondern um das physische Erleben von Effekten. „Meine Filme“, hat George Lucas geschrieben, „sind Achterbahnfahrten in Freizeitparks ähnlicher als einem Theaterstück oder einem Roman“ (Time v. 15. Juni 1981, zit.n. Jullier 1997, 37). Die „natürlichen Bedingungen“, auf deren Rolle ich im letzten Kapitel hingewiesen habe, spielen hier eine entscheidende Rolle.

Definition des energetischen Modus:

- räumliche Ebene: Konstruktion eines Bild-Ton-Raums (vs. eine Welt oder einem Raum des Spektakels);

- diskursive Ebene: (teilweise) Blockade der Produktion eines Diskurses zugunsten einer Konstruktion, die auf Variationen des Rhythmus und der Intensität basiert;

- affektive Ebene: Beziehungen, die eher auf Effekten als auf Affekten beruhen;

- enunziative Ebene: Konstruktion eines fiktiven Enunziators. 
Sehr wahrscheinlich finden sich im Videoclip die reinsten Manifestationen dieses Modus: Viele Clips erzählen keine Geschichte, sondern reißen mich mit in ein kunstvolles Vorüberziehen von Bildern. Dabei werden alle Ressourcen der filmischen Sprache mobilisiert, alle Tricks, damit ich aufs Engste am Rhythmus und an der Atmosphäre des Stücks haften bleibe. Viele Zuhörer, die kein musikalisches Hören erlernt haben (das im Gegenteil eine besondere Aufmerksamkeit für die Arbeit am Klang erfordert) empfangen auch die Musik, die im Radio läuft, in diesem Modus. Der energetische Modus drängt sich mir auch auf, wenn ich abends müde und mit halbem Auge fernsehe, ohne dem Gesagten oder der erzählten Geschichte große Aufmerksamkeit zu schenken, wenn ich mich vom Fluss der Bilder und Töne tragen lasse (daher kommt die Rede vom „flow“). Schließlich kommt es auch vor, dass ich die Welt (die Bewegung der Wolken, das fließende Wasser in einem Strom, das Feuer im Kamin, das unaufhörliche Vorbeiziehen der Autos auf der Autobahn) in diesem Modus betrachte.

\section{Dokumentarisierender Modus, moralisierender Modus}

Der Gegensatz zur Erfahrung der Fiktion regt den Theoretiker zur Konstruktion eines weiteren großen Modus an: des Modus der Erfahrung der Wirklichkeit. In früheren Veröffentlichungen habe ich dafür den Ausdruck dokumentarisierender Modus gewählt. Heute glaube ich, dass es nötig ist, nicht nur einen, sondern zwei Modi zu konstruieren, um dieser Erfahrung der Wirklichkeit gerecht zu werden. Dokumentarisierend soll weiter der Modus heißen, in dem man Informationen über die Wirklichkeit kommuniziert (und sei es nur, indem man sie zeigt), während ich von einem moralisierenden Modus sprechen werde, wo es um die Übertragung und/oder die Diskussion von Werten geht (auch Werte sind Teile der Realität). Wenn diese beiden Modi im Kommunikationsprozess auch oft verschränkt sind, hat man es doch mit zwei unterschiedlichen Erfahrungstypen zu tun: die Welt kennenlernen, Werte hinterfragen. Die beiden Modi sind auch Gegenstand unterschiedlicher Institutionalisierung geworden: auf der einen Seite die Wissenschaft, auf der anderen die Philosophie und die Moral. Es scheint daher angemessen, beide Modi zu trennen. 
Ich schlage die Ausgabe vom 13. August 2009 der Zeitung Le Monde auf. Mit Ausnahme einer kleinen Sektion, die Spielen (wie Kreuzworträtsel und Sudoku) gewidmet ist und die den ludischen Modus erfordert (ein Modus, der eine eigene Analyse verdient), wird schnell deutlich, dass der hier aufgebaute Kommunikationsraum in erster Linie einen Rückgriff auf den dokumentarisierenden Modus erfordert. Die Mehrheit der Artikel hat eine „diskursive“ Struktur, die mehr oder weniger stark mit narrativen Elementen oder Minierzählungen vermischt ist. Einige Artikel sind klar narrativ, vor allem die Biografien, seien es Nachrufe (wie auf Thierry Jonquet, Francisque Collomb, François Luchaire) oder nicht (wie bei Greg Louganis, dem vierfachen Olympiasieger). Auf der letzten Seite kann ich sogar einen Reisebericht lesen, dessen Titel eine direkte Referenz auf Jules Verne ist: „In 80 Tagen auf dem Fahrrad um die Welt“. Der Text mobilisiert drei der vier Prozesse der Fiktionalisierung: Diegetisierung, Narration und Mise en phase. Der einzige Unterschied zur Fiktionalisierung besteht darin, dass ich aufgefordert werde, einen realen Enunziator zu konstruieren: Guillaume Prébois, Radfahrer und Autor des Artikels. Es ist diese enunziative Konstruktion, die bewirkt, dass ich mich im dokumentarisierenden und nicht im fiktionalisierenden Modus befinde.

Einige Artikel sind Teil einer Serie, wie der über Greg Lougaris (die Serie trägt den Titel „Porträts des Sommers“) oder „Clarksdale und der Fluch der Baumwolle“ (die Serie heißt „Auf der Route 61“). Aber es finden sich auch Karten (z.B. um Clarksdale auf der Route $61 \mathrm{zu}$ lokalisieren oder für die Wettervorhersage), Tabellen (Radio- und Fernsehprogramm, wiederum der Wetterbericht), ein Faksimile der ersten Seite von Le Monde des 4. Mai 1989, Fotografien, eine Seite im Modus der Liste („Notizen“: Geburten, Todesnachrichten etc.).

Aber Le Monde fordert mich auch auf, den moralisierenden Modus zu verwenden. Das Editorial etwa hat die Form eines Diskurses, der versucht zu zeigen, dass die Werte des Friedens heute im Nahen Osten erfolgreich sein könnten. Auch die Zeichnung von Plantu, unten rechts auf der ersten Seite, erfordert eine solche Lektüre: Sie zeigt einen mit Gräbern und Leichen bedeckten Ort. Offensichtlich handelt es sich um gerade erst Getötete, denn sie bluten noch. Darunter steht: „Friedhof der Menschenrechte in Russland“. Im Vordergrund ist ein vornehm 
und westlich gekleideter Mann zu sehen, der einen Koffer in den Farben Europas (Sterne auf blauem Grund) in der Hand hält und in ein großes Rohr hineinblickt, das sich bis zum Horizont schlängelt und das ein Schild als russische Gasfernleitung ausweist. Eine Gedankenblase verrät, was der gutgekleidete Europäer denkt: „Ich würde ja verurteilen, aber ich sehe Licht am Ende des Tunnels!“. Ein moralischer „Diskurs“ in bildlicher Form.

Im Vergleich zur Fiktionalisierung wirkt die Diversität der Strukturen und Formen, die mit dem dokumentarisierenden und dem moralisierenden Modus vereinbar sind, erstaunlich. Die Besonderheit dieser Modi besteht tatsächlich darin, nur wenige Einschränkungen zu machen:

- auf der diskursiven Ebene sind alle diskursiven Formen akzeptiert: die häufigsten sind Erzählung und „Diskurs“;

- auf der räumlichen Ebene ist nur die Erzeugung eines plastischen (abstrakten) Raums untersagt, da ein solcher Raum den diskursiven Prozess blockiert; es besteht dagegen die Wahl zwischen verschiedenen Modalitäten der Konstruktion eines „diskursiven“ Raums (Tabellen, Symbolketten, Schemata, Karikaturen) oder der Konstruktion einer Welt;

- auf der Ebene der affektiven Beziehungen findet man sowohl eine Mobilisierung der narrativen Mise en phase als auch eine völlige Abwesenheit eines derartigen Mechanismus und damit das Risiko den Rezipienten zu langweilen (wie in manchen Lehrfilmen).

Von der Produktion von Informationen und Werten abgesehen betrifft die einzige Bedingung, die den Gebrauch dieser Modi einschränkt, die enunziative Ebene:

- beim dokumentarisierenden Modus die Konstruktion eines realen Enunziators, der hinsichtlich seiner Identität, seines Tuns und seiner Wahrhaftigkeit befragbar ist;

- beim moralisierenden Modus die Konstruktion eines realen Enunziators, der hinsichtlich seiner Identität, seines Tuns und seiner Werte befragbar ist. 
Beispiel für den dokumentarisierenden Modus: Wer bist $\mathrm{Du}$, dass Du mir von dem und dem Thema etwas erzählen willst? Welche besonderen Kompetenzen hast Du in dieser Frage, was hast Du getan, um die Informationen zu bekommen? Entspricht Deine Erzählung den Fakten, verheimlichst $\mathrm{Du}$ nichts? Beispiel für den moralisierenden Modus: Hast $\mathrm{Du}$ die Autorität, um die Behauptungen $\mathrm{zu}$ machen, die $\mathrm{Du}$ machst? Wer bist $\mathrm{Du}$, mich zu belehren? Willst $\mathrm{Du}$, dass ich diesen oder jenen Wert akzeptiere?

Dass der Enunziator befragbar ist, heißt nicht, dass er auch tatsächlich befragt wird. Es kommt oft vor, dass ein realer Enunziator konstruiert wird, ohne dass das Bedürfnis weiterer Nachforschungen und Befragungen besteht. Aber die Möglichkeit bestünde und das ist das Entscheidende. In jedem Moment können solche Fragen auftauchen und den Enunziator in Bedrängnis bringen. Diese Möglichkeit bildet die Grundlage des realen Enunziators.

Diese enunziative Bedingung eröffnet selbst einige Möglichkeiten: Tatsächlich können nämlich für ein und dieselbe Produktion verschiedene reale Enunziatoren konstruiert werden. Wenn ich etwa THE BIG SKY (Howard Hawks, USA 1952) gucke, kann ich als reale, dokumentarisierende Enunziatoren Hawks selbst (was sagt mir der Film über Hawks als Person?), die Epoche, in der er gedreht wurde (der Historiker Marc Ferro hat viel in diese Richtung gearbeitet), die filmische Sprache, das Kino der 1950er Jahre etc. konstruieren und als reale, moralisierende Enunziatoren: wiederum Hawks, das Produktionsstudio, Hollywood, die amerikanische Gesellschaft und deren Wertesystem etc.

Diese große Freiheit in der Konstruktion kann die Schwierigkeit erklären, die Produktionen, die diese Modi aktivieren, damit haben, im sozialen Raum angenommen zu werden: Während ich bei der Lektüre einer Fiktion ziemlich genau weiß, mit welcher kognitiven und affektiven Arbeit ich zu tun haben werde, ist dies bei diesen Produktionen nicht der Fall; diese Modi erzeugen keine starken Erwartungen, was für den Rezipienten nicht sehr angenehm ist.

Zum Beweis mittels Umkehrschluss lässt sich darauf hinweisen, dass die dokumentarisierenden und moralisierenden Produktionen, die vom Publikum die höchste Akzeptanz erfahren, solche sind, die 
sich entweder in der Form der Fiktion annähern oder die die Schwäche ihres Erwartungssystems durch eine starke Kontextualisierung kompensieren, die einen von beiden oder beide Modi aktivieren. Das passiert, wenn ich eine Tageszeitung wie Le Monde kaufe und dafür sorgt auch die Programmstruktur des Fernsehens. Für Dokumentarfilme gilt, dass sie am besten an für sie vorgesehenen Orten gezeigt werden (Festivals, feste Sendeplätze, klar angekündigte Ausstrahlungen) oder an Orten, die ihrerseits dokumentarisierende und moralisierende Funktionen haben (wie zum Beispiel die Schule). Allgemein lässt sich sagen, dass diese Produktionen desto besser vom Publikum angenommen werden, je mehr sie sich in aktuelle Debatten einmischen, also in bereits in der sozialen Sphäre bestehende dokumentarisierende und moralisierende Kommunikationsräume: Debatten über Sexualität, Debatten über Umweltfragen, über die Globalisierung etc.

Eine Konsequenz der Konstruktion eines realen Enunziators ist schließlich, dass ich selbst als reale Person in die Kommunikation eingebunden bin. Ein positives Resultat davon ist, dass ich meinen Widerspruch zum Enunziator markieren, seine Informationen in Frage stellen, seine Werte ablehnen kann, was bei Fiktionen viel schwieriger ist. Aber das hat auch seine Kehrseite: Ich werde selbst in Frage gestellt, muss mich erklären oder rechtfertigen. Dies erzeugt im wissenschaftlichen und moralischen Kommunikationsraum normalerweise keine Schwierigkeiten, weil wir es dort mit Akteuren zu tun haben (Wissenschaftler, Philosophen), die das Risiko, in diesem Raum zu agieren, bewusst auf sich genommen haben. Es lässt sich in dieser enunziativen Konstruktion aber einer der Gründe dafür sehen, dass dokumentarische und moralisierende Produktionen beim breiten Publikum weniger populär sind als fiktionalisierende. Während mich die Fiktion in Sicherheit wiegt (hier bin nicht ich als reale Person angesprochen), richten sich diese Produktionen an mich persönlich.

Definition des dokumentarisierenden Modus:

- diskursive Ebene: Produktion von Informationen (ohne Einschränkung der Form);

- affektive Ebene: nicht festgelegt; 
- enunziative Ebene: Konstruktion eines realen Enunziators, der hinsichtlich Identität, Tun und Wahrheit befragbar ist.

Definition des moralisierenden Modus:

- diskursive Ebene: Produktion von Informationen (ohne Einschränkung der Form);

- affektive Ebene: nicht festgelegt;

- enunziative Ebene: Konstruktion eines realen Enunziators, der hinsichtlich Identität, Tun und Werten befragbar ist.

\section{Rückkehr zum fiktionalisierenden Modus, fabularisierender Modus}

Die Weise, in der ich bislang die Modi dargestellt habe, lässt vermuten, dass ein Gegensatz zwischen dem dokumentarisierenden und dem moralisierenden, die eine Beziehung zur Wirklichkeit haben, auf der einen Seite und dem fiktionalisierenden Modus, der eine Nicht-Beziehung zur Wirklichkeit hat, auf der anderen Seite besteht.

Wenn auch diese Opposition in Analysen von Spiel- und Dokumentarfilmen oft behauptet wird, entspricht sie nicht der Erfahrung von Fiktionen. Denn die fiktionale Erfahrung ist auch eine Erfahrung der Wirklichkeit, einerseits als Kennenlernen der Welt (wieviele afrikanische Kinder haben beispielsweise Schnee, oder zu einer bestimmten Zeit, Supermärkte, zum ersten Mal in Spielfilmen gesehen), andererseits als Ort der Manifestierung von Werten: Wenn man in der Analyse des fiktionalisierenden Modus konsequent ist, kommt man immer irgendwann zu dem Punkt, wo er in einen „Diskurs“ mündet und in eine Moral, die er uns kommuniziert. Das hängt mit der Natur der Erzählung zusammen, die stets Werte transportiert, mit denen uns die Fiktion, über die Mise en phase, in Beziehung setzt und mit denen sich zu identifizieren sie uns einlädt.

Daher stellt sich die folgende Frage: Wenn es wahr ist, dass jede Fiktion sowohl dokumentarisierend als auch moralisierend ist, warum sollten wir dann überhaupt die Fiktionalisierung gesondert behandeln? Sollte sie nicht eher als ein Ensemble von Prozessen betrachtet 
werden, das vom dokumentarisierenden und moralisierenden Modus aktiviert werden kann, also als eine Modalität dieser Modi?

Eine solche Auffassung ist in der Tat nicht uninteressant. Wenn man bedenkt, dass die Fiktion auf dem dokumentarisierenden und dem moralisierenden Modus beruht, so zeigt dies, dass das Spiel der Fiktion nicht unschuldig ist: Sie wirkt einerseits informativ (und kann daher auch zu pädagogischen Zwecken eingesetzt werden), andererseits transportiert sie Werte - und hier ist Wachsamkeit gefragt. Das Problem einer solchen Analyse besteht jedoch darin, dass sie nicht widerspiegelt, wie die Fiktion erlebt wird: Die fiktionale Erfahrung unterscheidet sich von der des dokumentarischen oder des moralischen „Diskurses“.

Zweiter Vorschlag: Es sollte eine obligatorische Beziehung zwischen dem fiktionalisierenden Modus auf der einen Seite und dem dokumentarisierenden und moralisierenden Modus auf der anderen eingeführt werden, um anzuerkennen, dass jeder Rückgriff auf den fiktionalisierenden Modus in die beiden anderen mündet. Aber auch diese Fassung ist nicht befriedigend, denn wenn die Fiktion auch Informationen und Werte transportiert, so doch nicht mittels derselben Prozesse wie der dokumentarisierende und der moralisierende Modus. Bei der Fiktion findet der Bezug zur Wirklichkeit durch die Konstruktion eines fiktiven Enunziators der Erzählung statt. Daraus folgt, dass der reale Enunziator von Informationen und Werten hier maskiert ist, versteckt, bis zu dem Punkt, dass der Rezipient meinen kann, er existiere gar nicht. Dies ist die Besonderheit und auch die Stärke der Fiktion: Die Informationen und Werte werden hier sozusagen auf implizite Weise vermittelt; der Rezipient nimmt sie unwillkürlich und sogar unwissentlich auf, nur in dem er sich für die Erzählung interessiert. Dies hat Louis Marin (1978) dazu gebracht zu sagen, „die Erzählung sei eine Falle“.

Dritter Vorschlag: Meine obengenannte Definition muss um die Prozesse der Beziehung zur Wirklichkeit erweitert werden. Nur diese Lösung erscheint mir überzeugend. Daher die folgende neue Charakterisierung des fiktionalisierenden Modus. 
Definition des fiktionalisierenden Modus (neuer Vorschlag):

- räumliche Ebene: Konstruktion einer Welt (diegetisieren);

- diskursive Ebene 1: Konstruktion einer Erzählung (narrativieren);

- diskursive Ebene 2: von der Erzählung ausgehende Konstruktion eines „Diskurses“, der Informationen und Werte transportiert;

- affektive Ebene: Mise en phase zu den erzählten Ereignissen und daher mit den vermittelten Werten;

- enunziative Ebene 1: Konstruktion eines fiktiven Enunziators der Erzählung und der Figuren (fiktivisieren);

- enunziative Ebene 2: Konstruktion eines realen Enunziators von Informationen und Werten, der hinter dem Schutzmantel des Vertrags der Fiktivisierung agiert.

Die Verdopplung der diskursiven und der enunziativen Ebene erlaubt es uns zu begreifen, wie die Fiktion mit der Wirklichkeit interagiert.

Aber die Dinge liegen noch ein bisschen komplizierter. Denn es existieren auch Texte, die ihre Fiktion explizit als Illustration eines moralischen „Diskurses“ anlegen. Bei einer solchen Struktur kommen der „Diskurs“ und die Werte, die er transportiert, logischerweise zuerst: Selbst wenn die „Moral der Geschichte“ erst am Ende aufgedeckt wird, versteht doch jeder, dass die vorherige Erzählung dazu dient, die Moral zu veranschaulichen. Dennoch verliert die Fiktion nicht ihre Wirkung: Sie ist es, die den Leser gefangen hält, die ihm Lust macht, den Text bis ans Ende zu verfolgen. Fiktion und „Diskurs“ sind hier also im Gleichgewicht, ein Gleichgewicht, das allerdings speziell und auch ein wenig prekär ist: Sobald sich der moralische „Diskurs“ zu stark bemerkbar macht, kippt die Kommunikation in den moralisierenden Modus, sobald die Welt und die Erzählung zu dominant werden, kippen wir in die Fiktionalisierung. Nur wenn das Gleichgewicht gewahrt bleibt, lässt sich dieser Erfahrungstyp weder mit dem der Fiktion verwechseln (die konstruierte Welt ist hier weniger dicht, weniger präsent), noch mit dem moralisierenden Modus: Die Moral leitet sich aus der erzählten Geschichte ab und es ist zuerst diese, die den Rezipienten interessiert. 
Wir könnten solche Produktionen entweder als Unterkategorie des moralisierenden Modus verstehen (die Fiktionalisierung wird als „diskursiver“ Prozess verstanden, um zu moralisieren) oder als Unterkategorie des fiktionalisierenden Modus, der ohnehin, wie oben gesehen, immer in eine implizite Moral mündet, eine Moral, die hier lediglich explizit gemacht wird. Beide Vorgehensweisen haben aber den Nachteil, nicht der Tatsache gerecht zu werden, dass diese Konfiguration eine spezifische Erfahrung mit sich bringt. Noch wichtiger ist aber die Tatsache (die im Übrigen recht erstaunlich ist), dass sich diese Konstruktion, die (wie wir sahen) auf einer ziemlich instabilen Struktur beruht, sich im Laufe der Geschichte institutionalisiert hat, bis zu dem Maße, dass man ihr einen eigenen Namen gegeben hat: Man spricht von „Fabeln“, „Parabeln“ oder „Gleichnissen“. Wir haben es also mit einem Erfahrungstyp zu tun, der in schriftlicher und mündlicher Literatur, aber auch im Theater, in der Malerei und sogar in der Bildhauerei (die Kapitelle romanischer Kirchen funktionieren oft in diesem Modus) eine lange Tradition hat. Auch manche Filme funktionieren in diesem Modus: NeIGHBouRs (Norman MacLaren, CAN 1952), THE BOY WITH GREEN HAIR (Joseph Losey, USA 1948). Daher habe ich mich entschieden, einen spezifischen Modus zu konstruieren: den fabularisierenden Modus.

Definition des fabularisierenden Modus:

- diskursive Ebene 1: Konstruktion eines „Diskurses“, der ein Wertesystem festlegt (Werte, die von der Erzählung transportiert werden);

- enunziative Ebene 1: Konstruktion eines realen Enunziators des „Diskurses“;

- räumliche Ebene: Konstruktion einer Welt (die aber oft weniger dicht ist als in einer Fiktion);

- diskursive Ebene 2: Konstruktion einer Erzählung;

- affektive Ebene: Aktivierung der Mise en phase durch die Erzählung;

- enunziative Ebene 2: Konstruktion eines fiktiven Enunziators der Erzählung. 
So beschrieben erscheint der fabularisierende Modus als aus denselben Prozessen zusammengesetzt wie der fiktionalisierende Modus, aber die Hierarchisierung der Prozesse ist eine andere. Während beim fiktionalisierenden Modus die Konstruktionen einer durch einen fiktiven Enunziator hervorgebrachten Erzählung das Ensemble dominiert, ist es hier die Konstruktion eines „Diskurses“ durch einen realen Enunziator. Methodologisch lässt sich daraus ableiten, dass sich die Modi nicht nur durch ihre Prozesse voneinander unterscheiden können, sondern auch durch ihre Struktur.

Man könnte bei der Beschreibung sicherlich noch präziser sein (im nächsten Kapitel findet sich beispielhaft eine detailliertere Charakterisierung zweier Modi: des künstlerischen und des ästhetischen), mir war aber zunächst wichtig, ein bestimmtes Vorgehen zu veranschaulichen. Für jeden Modus habe ich eine systematische Beschreibung in Form der Struktur der Prozesse geliefert. Man mag mit meinen Definitionen nicht einverstanden sein, aber zumindest kann jeder in Kenntnis der Gründe reagieren. Ein anderer Vorteil dieser systematischen Beschreibungen liegt darin, dass sie einen Vergleich der verschiedenen Modi untereinander erlauben und auf diese Weise, durch die Differenzierung, den Typus der kommunikativen Erfahrung erkennen lassen, den sie bezeichnen. Und schließlich hoffe ich gezeigt zu haben, dass die Arbeit der Konstruktion der Modi in sich interessant ist, und zwar aufgrund der theoretischen Fragen, zu denen sie führt. 
[Schema 4]

\begin{tabular}{|c|c|}
\hline Raum der Sendung (S) & Raum der Rezeption (R) \\
\hline Allgemeine Bedingungen \\
\hline Sprachliche Bedingungen \\
Kommunikative Kompetenz als \\
Reservoir von Prozessen \\
Modi \\
Kommunikationsräume \\
Auswahl der Prozesse, des oder der \\
zu benutzenden Modi
\end{tabular}




\section{Kapitel 3}

\section{Ästhetischer Modus, künstlerischer Modus}

\section{Beziehungen zwischen Modi und Räumen}

Die im letzten Kapitel konstruierten Modi lassen sich durch ihre vertikale Struktur charakterisieren: Sie bestehen aus einer Reihe von Prozessen, die gleichzeitig und in einem Stück zustande kommen. Sie können zwar hierarchisiert und auch dauerhaft einzeln angewandt werden, aber ihr System ist nicht zeitlich organisiert: Sie werden alle gemeinsam mobilisiert, als ein unauflösbares Ganzes. Die künstlerischen und ästhetischen Modi erfordern dagegen, einer horizontalen Struktur folgend konstruiert zu werden: Ihre Prozesse organisieren sich zeitlich, in einer Abfolge. Während ich nicht nur teilweise fiktionalisieren oder dokumentarisieren kann (entweder fiktionalisiere ich oder ich dokumentarisiere oder ich mobilisiere einen anderen Modus), kann ich eine ästhetische oder künstlerische Lektüre auch betreiben, ohne sie zu vervollständigen.

Zwar erkennen viele Theoretiker die Autonomie des ästhetischen Modus an (so kann man ein natürliches Objekt im ästhetischen Modus betrachten, jedoch nicht im künstlerischen), aber meistens wird der ästhetische so eng mit dem künstlerischen Modus verbunden, dass er ihm als Definition dient. „Wenn die ästhetische Erfahrung ein intrinsisches Ziel und Wert konstituiert, dann mag es gute Gründe geben, Kunst auf dieser Grundlage zu definieren [...]“ (Shusterman 1994 [1992], 45). Ebenso bemerkt der Anthropologe Jacques Maquet in The Aesthetic Experience (1986), dass in Europa seit der Renaissance ein Objekt dann als Kunst gilt, wenn es als einzige Funktion die des ästhetischen Genusses hat (wobei er den Genuss dem gegenüberstellt, was er Ästhetisierung des Funktionalen nennt). Rainer Rochlitz 
bestätigt seinerseits, dass ,jedes (Kunst-)Werk zunächst auf ästhetische Anerkennung“ abzielt $(1994,36)$ und widmet sich im vierten Kapitel seines Buches einer Bestimmung der „ästhetischen Kriterien“ des Künstlerischen. Gérard Genette wiederum ist zwar der Überzeugung, dass es unmöglich sei, solche Kriterien zu bestimmen, definiert aber dennoch jede künstlerische Produktion „als Artefakt (menschliches Produkt) mit ästhetischer Funktion“ (1994, 10) und beteuert, das Vorhandensein einer ästhetischen Intention genüge (und sogar ein „Quäntchen ästhetischer Intention“), um aus jedem Objekt ein Kunstwerk zu machen $(1997,271)$.

Angesichts dieser Positionen scheint es mir im Gegenteil notwendig, die beiden Modi als komplett unabhängig voneinander zu konstruieren. Im Unterschied zu Jean-Marie Schaeffer (1992, 28-31), ist diese Position für mich keine philosophische Position; mir scheint einfach, dass man, indem man diese Unabhängigkeit annimmt, am besten gewappnet ist, um einer künstlerischen und/oder ästhetischen Kommunikationserfahrung Rechnung zu tragen.

\section{Vom Modus zu den ästhetischen Räumen}

Im Gegensatz zu dem was ich anderswo geschrieben habe (Odin 2002), glaube ich nicht mehr, dass der ästhetische Modus durch die Tatsache charakterisiert ist, dass er keine Konstruktion eines Enunziators beinhalte. Was mich zu dieser unglücklichen Idee geführt hatte, war, dass ich nicht herausbekam, worin der Enunziator natürlicher Objekte bestehen könne, wenn sie im ästhetischen Modus gelesen werden zum Beispiel Steine, wie in dem schönen Buch, das Roger Caillois (2004 [1970]) ihnen gewidmet hat; aber, abgesehen davon, dass es keinen Text ohne Enunziator geben kann, ist mir bewusst geworden, dass ich bei einer derartigen Lektüre selbst als Enunziator des ästhetischen Textes fungiere. Zu dem Fehler verleitet hat mich also das, was Gérard Genette die Operation der „Objektivierung des Subjektiven“ nennt (1997, 117): das Objekt der ästhetischen Lektüre wird betrachtet, als hätte es die vorgefundenen ästhetischen Werte selbst inne, dabei bin in Wirklichkeit ich es, der sie ihm im Laufe der Lektüre zugeschrieben hat. Die Vorstellung, es gebe keinen Enunziator, rührte daher, dass mir 
dieser Schritt der Zuschreibung nicht bewusst war. Ich bin es also, der den ästhetischen Text konstruiert - es handelt sich um die Konstruktion eines realen, hinsichtlich der Identität befragbaren Enunziators und es ist sogar diese enunziative Struktur, die ein wesentliches Charakteristikum des ästhetischen Modus ausmacht.

Ich nenne ästhetischen Modus jenes Vorgehen, durch das ein Subjekt sich auf ein Abenteuer einlässt, das in der Suche nach ästhetischen Werten besteht.

Um dieser Suche Rechnung zu tragen, stelle ich die Hypothese auf, dass ich, als Subjekt des ästhetischen Modus, mich wie das Subjekt eines narrativen Parcours verhalte, der die Entdeckung ästhetischer Werte zum Ziel hat. Der Status dieses narrativen Parcours muss jedoch genau präzisiert werden: Man darf ihn keinesfalls mit narrativen Konstruktionen auf der diskursiven Ebene des ästhetischen Modus selbst verwechseln; es geht hier vielmehr darum, mich des narrativen Modells als einem Werkzeug der Meta-Ebene zu bedienen, um die Dynamik des Modus selbst zu beschreiben.

Die erste Phase besteht in dem Moment meiner Begegnung mit dem Objekt, den man in der Terminologie der narrativen Semiotik von Greimas (1971 [1966]), die ich während der gesamten Analyse verwenden werde, als ,Vertragsmoment bezeichnen kann. Es handelt sich um einen Vertrag, der mich dem ästhetischen Modus gegenüber verpflichtet. Dieser Vertrag kann verschiede Formen annehmen: Manchmal ist es das Objekt, das in mir den Wunsch weckt, mich auf die Suche nach Werten zu begeben: „Oh, wie ist das schön!“ (das ist Liebe auf den ersten Blick); manchmal ist es ein Dritter, der meine Aufmerksamkeit auf das Objekt lenkt („Schau, wie schön das ist!“) oder ein kritischer Text oder eine Lektüre; manchmal ist es ein komplexer Prozess, den man nur von Fall zu Fall beschreiben kann. Eine Sache ist sicher: Ohne affektive Beziehungen zum Objekt besteht keine Chance, dass ich mich dem ästhetischen Modus gegenüber verpflichtet fühle. Affektive Beziehungen stellen den Motor (den Sender) des ästhetischen Modus dar, der die Suche anstößt, der meinen Wunsch weckt, weiter nach Werten zu suchen.

Die zweite Phase - die „qualifizierende Sequenz“ - entspricht der Zuschreibung von Möglichkeiten (Helfer), die mir erlauben, die Suche 
durchzuführen. Der ästhetische Modus funktioniert in der Tat nur unter bestimmten Bedingungen (die Möglichkeit, sich von dringenden Alltagsangelegenheiten loszusagen, Verfügbarkeit...) und fordert von Seiten des Subjekts bestimmte Eigenschaften, auch wenn sich keine Einigkeit über die Liste dieser Eigenschaften herstellen lässt (Empfindsamkeit, Fähigkeit zur Versinnbildlichung, Vorstellungskraft...). Worum es hier geht, ist eine Frage der ästhetischen Erziehung, eine gänzlich andere Frage als die der künstlerischen Erziehung, mit der sie zu oft verwechselt wird.

Die abschließende Phase (die „Hauptsequenz“) besteht in der (Nicht-) Produktion von ästhetischen Werten, vorausgesetzt diverse Gegner konnten ausgeschaltet werden, nämlich alles, was mich von meiner ästhetischen Suche ablenkt. Ich kann übrigens mein eigener Gegner sein und mich für ganz andere Dinge interessieren als für die Suche nach ästhetischen Werten des Objekts (vermittelte Informationen, die erzählte Geschichte, den Autor ...).

Ästhetische Werte dürfen nicht mit der Botschaft des Werkes verwechselt werden (es sind weder von der Geschichte transportierte Werte, noch Werte des vorgetragenen „Diskurses“). Ästhetische Werte bilden sich während meines Zusammentreffens mit dem Objekt, das einer doppelten Bewegung folgt: von den empfundenen Emotionen zur kognitiven Arbeit, die sie auslösen und/oder von der verrichteten kognitiven Arbeit zu den Emotionen, die diese auslöst. Wenn ich auch mit der ersten Bewegung mit Nelson Goodman übereinstimme, für den „Emotionen in der ästhetischen Erfahrung kognitiv funktionieren“ (1997 [1976], 228), so dreht die zweite Bewegung Goodmans Formulierung um, um zu behaupten, dass in der ästhetischen Erfahrung kognitive Produktionen affektiv verlaufen. Die beiden Bewegungen erscheinen mir untrennbar miteinander vereint, selbst wenn die eine die andere unter bestimmten Bedingungen in den Hintergrund drängen kann.

Wie dem auch sei, die wesentliche Arbeit des ästhetischen Modus findet auf der enunziativen und affektiven Ebene statt. Auf diskursiver Ebene bleibt die Produktion Sache des Subjekts, die sich nur in Form eines „inneren Diskurses“ manifestiert. Sobald sie aus sich herausgeht, sobald sie Anlass für die Produktion von Aussagen gibt, kann sie ganz 
verschiedene Formen annehmen. Daher sieht man in einem Reiseamateurfilm eine Frau mitten in einer wunderschönen Landschaft; sie ist allein (wie so oft ist es der Ehemann, der sie filmt) und sie gestikuliert wild mit beiden Armen, einerseits um ihrem Mann zu zeigen, was er filmen soll (die Wasserfälle, die sie umgeben) und um demjenigen, der später den Film sehen wird, mitzuteilen: „Schau, wie schön das ist!“; es wird kein Wort gesprochen (der Film ist stumm), aber die Gesten werden zum Diskurs (Sierek 1995). Die diskursive Produktion kann vom einfachen Ausdruck der Bewunderung („Oh!“) bis zur mehrbändigen ästhetischen Abhandlung gehen, über ein Gedicht, dass meine ästhetische Erfahrung preist, die Erzählung meiner Begegnung mit dem Objekt, über die Produktion von Zeichnungen, Gemälden, Fotografien, Filmen und sogar über Musik (Bilder einer Ausstellung von Mussorgski zielt darauf ab, uns an der ästhetischen Erfahrung des Autors mit den Gemälden Victor Hartmanns teilhaben $\mathrm{zu}$ lassen). Entscheidend ist allein, dass die diskursive Struktur die Kommunikation ästhetischer Werte betrifft. Allerdings muss man aufpassen, den Ausdruck „Wert“ nicht ausschließlich „diskursiv“ zu verstehen. Sicher, Werte haben immer einen „diskursiven“ Inhalt, das Entscheidende liegt aber oft woanders, in den sinnlichen Beziehungen, die sie manifestieren. Beim ästhetischen Modell geht es nicht nur darum, einen Text zu konstruieren, sondern darum, eine „Aufteilung des Sinnlichen“ auszulösen, um eine Formulierung von Jacques Rancière aufzugreifen (2006 [2000]). Sicher, ich habe in meinen Analysen bereits den Platz auf der affektiven Ebene vorgesehen, aber man muss noch weitergehen: Der ästhetische Modus lädt ein, zuzugeben, dass eine bestimmte Überlegenheit, ja sogar eine bestimmte Selbstständigkeit des Sinnlichen in Bezug auf die textuelle Konstruktion möglich ist (Herman Parret [1993] spricht in dieser Hinsicht von einer Ästhetisierung der Pragmatik).

Wie dem auch sei, im Zentrum des ästhetischen Modus befinden sich Werte; sie rechtfertigen ihn letztlich. Diese Werte gehören zum Kollektiv; sie drängen sich dem Individuum auf. Der ästhetische Kommunikationsraum regelt die ästhetischen Beziehungen seiner Mitglieder zu den Werken und zur Welt. Der Inhalt der ästhetischen Werte (welche die Relevanzkriterien bilden, die jedem ästhetischen 
Kommunikationsraum zu Kohärenz verhelfen) variiert von einem Raum zum nächsten: Schönheit, Hässlichkeit, Erhabenheit, wohl geraten, neu, authentisch, Widerstandsfähigkeit, Fähigkeit zur Lebensumstellung, Entwicklung der Empfindsamkeit, Bereicherung des Ichs und der Gesellschaft etc. Diese Werte verändern sich nicht nur im Laufe der Geschichte (es gibt eine Geschichte der ästhetischen Werte), es kommen zu einem gegebenen Zeitpunkt auch mehrere ästhetische Räume vor. Es sind diese Räume, die Pierre Bourdieu in Die feinen Unterschiede (1982 [1979]) gegeneinander abgrenzt, indem er die Dispositionen der Konsumenten, die sie ihrer Position im ökonomischen Feld verdanken, als Kriterien nutzt. Es gibt also eine Diachronie und Synchronie der ästhetischen Kommunikationsräume, aber hierzu kann die Semiopragmatik wenig beitragen. Wenn sie auch den Rahmen der Analyse liefert, so kommt es, sobald man zu den Inhalten übergeht, Historikern und Soziologen $\mathrm{zu}$, die Arbeit $\mathrm{zu}$ erledigen: Sie müssen den semantischen Einsatz der Werte erforschen, der diese Räume definiert.

Definition des ästhetischen Modus

Phase 1: Vertrag

- affektive Ebene: Subjekt und Objekt werden in Beziehung gesetzt;

- enunziative Ebene: Ich entwerfe mich als Subjekt, das sich auf die Suche nach ästhetischen Werten des Objekts begibt;

Phase 2: qualifizierende Sequenz

- affektive Ebene: affektive Erfahrung mit dem Objekt;

- enunziative Ebene: Suche nach Zusatzmitteln, die mir helfen können, Gegner zu beseitigen;

Phase 3: abschließende Sequenz

- affektive Ebene: unbestimmte Form; Produktion ästhetischer Werte;

- diskursive Ebene: unbestimmte Form; Produktion ästhetischer Werte. 


\section{Vom künstlerischen Modus (reduzierte Form) zur Einschreibung in den Raum der Kunst}

Im Laufe des Jahres 1908 (etwas vor dem berühmten Text von Ricciotto Canudo über „die siebte Kunst“), versuchen ein Anwalt, Émile Maugras (zugleich delegierter Verwalter von Omnia-Pathé und Präsident des Verwaltungsrats des Kinotheaters Pathé) und ein Doktor der Rechtswissenschaft (zugleich Verwalter des Nationalkinos Pathé), Maurice Guégan, das Kino in den Raum der Kunst zu überführen, um die Produktionen der Firma, für die sie arbeiten, aufzuwerten, also aus Gründen ökonomischer Konkurrenz. ${ }^{1}$ Ihre These wird in Le Cinématographe devant le droit (1908) dargelegt. ${ }^{2}$ Die gesamte Darlegung der beiden Autoren beruht auf einem einzigen Argument: Es geht darum $\mathrm{zu}$ beweisen, dass das Kino sich in den Rahmen des Gesetzes über künstlerisches Eigentum von 1793 einfügt, das besagt: „Urheber aller Art, Komponisten, Maler und Zeichner, die Bilder oder Zeichnungen anfertigen lassen, behalten ihr ganzes Leben lang das Exklusivrecht, ihre Werke zu verkaufen, verkaufen $\mathrm{zu}$ lassen, zu vertreiben [...]“ (Maugras/Guégan 1908, 2f).

Der Beweis besteht zunächst einmal darin, zu zeigen, dass das Kino nichts anderes als eine Zeichnung ist: „Einer allgemeingültigen Definition zufolge ist eine Zeichnung die Repräsentation einer oder mehrerer Abbildungen einer Landschaft, eines beliebigen Objekts, angefertigt mit einem Stift, einer Feder oder einem Pinsel oder durch eine andere Möglichkeit (Trousset). Der Kinematograph ist nichts anderes als die Repräsentation von Landschaften oder Personen mittels eines mechanischen und chemischen Prozesses“ (ibid., 3). Selbst wenn eine ganze Tradition die Zeichnung in Zusammenhang mit „mechanischen Künsten“ gestellt hat, selbst wenn der Gebrauch

1 Im Allgemeinen wird der Artikel von Ricciotto Canudo „Trionfo del Cinematografo“, erschienen am 25. November 1908 in der Nuovo Giornale de Florence (Canudo 2016 [1908/1911]), als Gründungstext des Kinos als Kunstform angesehen. Das Werk von Maugras und Guégan scheint vom Anfang desselben Jahres zu stammen und wurde damit etwas früher verfasst als der Artikel von Canudo.

2 Der folgende Abschnitt gründet auf einer gemeinsam mit André Gaudreault durchgeführten Recherche; im Übrigen war es Gaudreault, der mich mit dem Text von Maugras und Guégan vertraut gemacht hat (Gaudreault \& Odin 2001). 
bestimmter Verfahren (Raster, optische Werkzeuge, Anwendung von Regeln der Perspektive und Geometrie ...) manchmal den menschlichen Eingriff begrenzt hat, so lässt diese Definition aus, was üblicherweise als Hauptmerkmal der Zeichnung angeführt wird, nämlich ihren „gestischen Charakter“: die Tatsache, dass sie durch „Werkzeuge, die seit jeher eine natürliche Verlängerung der menschlichen Hand waren“ (Larousse) hergestellt wird. Maugras und Guégan scheint dieses Problem bewusst zu sein, denn sie achten darauf, ihre Definition mit doppelter Aussagekraft auszustatten: Einerseits präsentieren sie sie als „allgemeingültig“, das heißt als von jedermann akzeptiert (die Doxa wird als Enunziator eingeführt); andererseits wird sie einer Autorität zugeschrieben: Trousset. Es handelt sich um Jules Trousset, der 1877 das Nouveau Dictionnaire Encyclopédique veröffentlicht hat. In Wirklichkeit beruht die Gleichsetzung von Kino und Zeichnung hauptsächlich auf dem letzten Teil der Definition, in dem es um die Repräsentation „durch eine andere Möglichkeit“ geht (hervorgehoben im Werk selbst).

Ein zweiter Beweisschritt setzt das Kino mit der Gravur gleich: „Die Gravur ist die Kunst, Figuren auf einer flachen Oberfläche zu produzieren, entweder durch Schnitte, für gewöhnlich wenig tief, oder mit Hilfe von Ätzungen oder schließlich durch Prozesse der Ziselierung und der Schnitzerei. Die Kinematographie reproduziert ebenfalls Bilder auf flachen Oberflächen (Filmrolle) mit Hilfe von Ätzungen (Entwicklerbad), denn man darf sich nicht täuschen, die durch das Licht bedruckte Filmrolle enthält praktisch das Bild; das Entwicklerbad ist nur das Ätzmittel, das, indem es bestimmte Salze zerstört, die Zeichnung erscheinen lässt. Die Kinematographie ist also eine Gravur [...]“ (Maugras/Guégan 1908, 4). An dieser Stelle ist der Gedankengang ganz besonders modern: Er geht in Richtung der Definition von Fotografie als Index, wie man sie bereits bei Charles S. Peirce (2000 [1865-1903]) findet und jüngst bei Jean-Marie Schaeffer, der von einem „Lichtabdruck“ spricht (1987, 59). Doch die Definition von der Fotografie als Index ist weit von dem entfernt, was sie zur Kunst macht, sondern zeichnet sie im Gegenteil als einen Abdruck der Wirklichkeit aus. Genau aufgrund ihres Status als Index sieht Schaeffer Fotografie als „prekäre Kunst“ an: „Wie soll man zwischen dem unterscheiden, 
was zum Bild, und dem, was zur Wirklichkeit gehört, wenn man einem Bild gegenübersteht, das seine Besonderheit daraus zieht, dass es als Aufnahme der Wirklichkeit wahrgenommen wird?" (ibid., 158). Die Gleichsetzung von Kino und Gravur ist also zumindest ein paradoxes Argument, um das Kino in den Raum der Kunst zu überführen.

Die Beweisführung ist also nicht frei von Schwächen, aber was für die beiden Autoren zählt, ist zu zeigen, dass das Kino dadurch, dass es Zeichnung und Gravur ist, in den Rahmen des Gesetzes von 1793 fällt und daher als zum Raum der Kunst zugehörig anerkannt werden muss: „Die Kinematographie ist eine Zeichnung oder Gravur und es ist sicher, was auch immer der Wert des Werkes sei, dass man sie zu den schönen Künsten rechnen muss“ (Maugras/Guégan 1908, 5; Herv. R.O.). Diese Aussage verdient zwei Anmerkungen. Zum einen ist der Preis der Einschreibung des Kinematographen in den Raum der Kunst die Negierung seiner medialen Besonderheit: „Die Kinematographie ist eine Zeichnung oder Gravur.“ Die zweite Anmerkung betrifft den eingeschobenen Satz; dieser betont eindeutig, dass der Prozess der Einschreibung eines Objektes in den Raum der Kunst (in diesem Falle des Kinos) nichts mit dem ästhetischen Wert des Objekts zu tun hat: „was auch immer der Wert des Werkes sei“ (Canudo dagegen verbindet den Eingang des Kinos in den Raum der Kunst aufs Engste mit einer ästhetischen Produktion).

Was hier die Einschreibung in den Raum der Kunst erlaubt, ist einzig und allein der Status des Enunziators (wir befinden uns im Paradigma der „institutionellen“ Definition der Kunst: Weitz 1956; Dickie 1988). Jeder Film ist also dem Raum der Kunst zuzurechnen, weil der Enunziator, der ihn hervorbringt, das Kino, diesem Raum angehört.

Jedes Objekt kann also ebenso als dem Raum der Kunst zugehörig betrachtet werden, wenn sein Enunziator selbst als dem Raum der Kunst zugehörig anerkannt ist: Das ist die Logik des ready made. Diese Logik funktioniert unabhängig davon, ob der Enunziator ein Individuum (Duchamp, Mozart), eine Institution (das Centre Pompidou), eine Gruppe (die Impressionisten), eine Form (Literatur) und sogar, wie wir gerade gesehen haben, eine Ausdrucksform (Zeichnung, Gravur) oder ein Medium (Kino) ist. Auf dieser Ebene bedeutet die Umsetzung des 
künstlerischen Modus, einen realen, dem Raum der Kunst angehörenden Enunziator zu konstruieren.

Es kommt vor, dass sich der künstlerische Modus auf diesen Prozess beschränkt. Sofern der Prozess dem Objekt selbst äußerlich bleibt (er beschränkt sich auf dessen Kennzeichnung) spreche ich von künstlerischer Kennzeichnung.

Definition des künstlerischen Modus (reduzierte Form)

- enunziative Ebene: Konstruktion eines dem Raum der Kunst angehörenden Enunziators (künstlerische Kennzeichnung).

Selbst wenn man der Meinung ist, dass diese Beschreibung des künstlerischen Modus zu kurz greift, sollte man ihre Bedeutung nicht unterschätzen. Einerseits weil diese Art des Vorgehens keineswegs ungewöhnlich ist: Es kommt sogar sehr häufig vor, dass ich dazu gebracht werde, den künstlerischen Status eines Objekts zu erkennen („das ist Kunst“), ohne mich weiter um eine Suche nach Werten, eine Analyse oder eine kontemplative Betrachtung dieses Objekts zu bemühen, entweder weil dieses Objekt mich nicht berührt (ich kann noch so sehr wissen, dass Poussin ein anerkannter Künstler ist, seine Gemälde sagen mir nichts), oder, noch einfacher, weil ich mir selbst nicht einmal die Frage stelle: Das ist Kunst, ich weiß, aber dabei bleibt es. Auf der anderen Seite zieht die Zuordnung eines Werkes zum Raum der Kunst beachtliche Konsequenzen nach sich: Es gewinnt an „Ansehen“, an „Aura“, verkauft sich zweifellos besser und wird dem Urheber Rechte einbringen. Es kann sogar vorkommen, dass dieses Label dazu anregt, es aufmerksamer zu studieren und schließlich zum vollständigen künstlerischen Modus führt, sogar zum ästhetischen Modus.

\section{Vom künstlerischen Modus (vollständige Form) zu den Räumen der Kunst}

Das Ziel des künstlerischen Modus in seiner vollständigen Form ist die Herstellung einer verbindlichen Beziehung zwischen den Eigen- 
schaften des Objekts und einem durch einen Eigennamen ausgewiesenen Enunziator: Das ist ein Renoir, ein Mozart, ein Werk von Le Corbusier.

Auf Anhieb wird eine doppelte Unterscheidung zum vorherigen Prozess erkennbar: Einerseits muss der Enunziator zwangsläufig individualisiert werden (ich kann mich zum Beispiel nicht damit begnügen, zu sagen, der Enunziator ist das Kino; Enunziatoren wären: Abel Gance, Jean Renoir oder Alain Resnais); andererseits ist man am Objekt und seinen Eigenschaften interessiert.

Die Bewegung, die das Objekt mit dem Eigennamen verbindet, kann in zwei Richtungen erfolgen: vom Eigennamen zum Objekt oder vom Objekt zum Eigennamen. In beiden Fällen ist es der Eigenname, der im Mittelpunkt der Verbindung zur Kunst steht. Daran denkt Ben, ein Maler aus Nizza, der für seine schwarz-weißen Malereien in Form von Aphorismen bekannt ist, wenn er sagt: „Kunst ist eine Frage des Eigennamens".

In dieser Hinsicht besteht die ganze Arbeit des künstlerischen Modus darin, die Stelle des Eigennamens zu füllen. Der Eigenname ist in der Tat ein Stellvertreter; Searle beschreibt ihn wie einen Nagel, an den man Beschreibungen hängt (1971 [1969]). Prozesse des künstlerischen Modus haben zur Aufgabe, einen Eigennamen mit Inhalten zu füllen, ob dies nun vor oder nach der Nennung des Eigennamens geschieht:

- biografische Recherchen;

- Recherchen zum Kontext, in dem das Werk produziert wurde (historische Recherchen);

- thematische und stilistische Recherchen, die darauf abzielen, das hervorzubringen, was die Werke eines Autors (desselben Namens) untereinander verbindet.

Der künstlerische Modus kann sich schließlich noch durch die Konstruktion von Serien entwickeln und durch den Vergleich mit Produktionen anderer Eigennamen, entweder synchron oder diachron (die Geschichte der Kunst ist eine Geschichte der Eigennamen). 
Der künstlerische Modus erscheint auf diese Weise, als würde er im Wesentlichen auf einem enunziativen Prozess beruhen (die Kennzeichnung oder Nennung eines Eigennamens) und auf diskursiven Prozessen. Wenn der ästhetische Modus eine Suche ist, dann ist der künstlerische Modus in seiner vollständigen Form eine Recherche. Auch wenn sie nicht verboten ist, eine affektive Produktion scheint hier nicht nötig. Ich kann ein Werk und seine Struktur sehr genau analysieren, ohne im Geringsten berührt oder bewegt zu sein und ohne mich auf die Suche nach ästhetischen Werten zu begeben. Pierre Sorlin unterscheidet auf diese Weise zwischen der „stilistischen“ und der „systemischen“ Analyse des ästhetischen Ansatzes (1992, 210-212). Zahlreiche Arbeiten der Kunstgeschichte, viele wissenschaftliche Schriften, Texte von hoher Qualität, die gut belegt und sogar von großer Bedeutung für die textuelle Arbeit sind, zeugen von keiner Suche nach ästhetischen Werten. Eine solche wird von der Institution auch nicht erwartet ...

Auf diese Weise bleibt der künstlerische Modus oft vom ästhetischen Modus getrennt, sogar wenn es sich um seine vollständige Form handelt.

Am Ende dieser Analyse scheint eine Sache sicher zu sein: In unserem Kulturraum garantiert einzig und allein die Nennung des Eigennamens eine vollständige Zugehörigkeit zum Raum der Kunst.

Arbeiten, die darauf abzielen, afrikanische Produktionen aus dem ethnografischen Raum in den Raum der Kunst zu überführen, belegen dies eindrücklich (dasselbe gilt für Produktionen aus Ozeanien oder von Inuits ...). Alles beginnt mit der Analyse der Objekte hinsichtlich einer Etablierung identifizierbarer Gruppen mit entsprechenden Merkmalen, die durch kategoriale (Masken, Skulpturen, Sitze ...), materielle (Holz, Stein, Metall ...) und stilistische Differenzierungen ausgemacht wurden. Diese Gruppen führen zur Konstruktion einer Reihe kollektiver Enunziatoren, üblicherweise verbunden mit dem Herkunftsort dieser Objekte (man spricht von Masken der Dogon, der Nigerianer, der Elfenbeinküste ...) und einem bestimmten Zeitpunkt (Bestimmung von Perioden). Weiter werden diese Gruppen je nach Stamm in Untergruppen unterteilt. Doch der große Moment, den alle Kunsthistoriker erwarten, ist die Identifizierung des Schöpfers, 
erkennbar an seiner „,individuellen Vision“ (Willett 2002 [1971/1993], 144) und versehen mit einem Eigennamen. Martine Degli und Marie Mauzé schreiben: „Lange hat man die Qualität der Künstler in den nicht-westlichen Gesellschaften bestritten, ob es sich um Bildhauer, Poeten oder Musiker handelte, der künstlerische Ausdruck wurde nur als besondere Emanation der Schaffenskraft der jeweiligen Gesellschaft verstanden [...]. Erst ab den 1960er Jahren haben einige wenige Forschungen die Aufmerksamkeit auf die individuelle Dimension der Kreation gelenkt, die über die stilistischen oder kulturellen Beschränkungen hinausweisen: Einige indigene Künstler haben so die Anonymität verlassen“ (Degli/Mauzé 2000, 12). Nachdem er bemerkt hat, dass die Benennung nach Stilen „ein großer Durchbruch“ sei, hält Frank Willett es für notwendig, zu ergänzen, „selbst wenn der Name des Bildhauers nicht aufgenommen wurde“. Und man spürt seine Erleichterung, präzisieren zu können, dass „heute hunderte Namen von Künstlern in der publizierten Literatur genannt werden" und einige Forschungsarbeiten nennen zu können, die sich auf identifizierte Bildhauer beziehen: die von Fischer zu Tame, Si, Tompieme und Son aus Nordost Liberia, die des Vaters Kevin Carroll zum Bildhauer Yoroubas Arowogun und seines Sohnes Bandele usw. (Willett 2002 [1971/1993], 204-219). Der fehlende Eigenname erzeugt eine Lücke. So erklärt sich die Freude, die man im Einleitungstext von Philippe Dagen über die Ausstellung Abomey (im Musée du quai Branly) spürt: „Endlich sind afrikanische Künstler nicht mehr anonym. Die Ausstellung Abomey, im Musée du quai Branly, versieht die Werke mit Namen“. Endlich „kann man mit afrikanischen Skulpturen das machen, was man mit europäischer Kunst macht: stilistische Vergleiche, Untersuchung eines Details von Einzelpersonen, ein Markenzeichen“ (Le Monde v. 29./30.11.2009).

Ein Beweis a contrario der Wichtigkeit von Eigennamen in unserem Raum der Kunst besteht in dem aktuellen Prozess der „Distinktion“ bei Sammlern primitiver Kunst, gerade die Abwesenheit eines Eigennamens aufzuwerten. Laut Sally Price erhöht „die Anonymität des Schöpfers den Wert eines Kunstwerks ...“. Sie zitiert die Absichten eines Pariser Sammlers: „Ich bin ganz entzückt von der Anonymität des Künstlers. Daß ich den Künstler nicht kenne, bereitet mir großes 
Vergnügen. Sobald man erfährt, wer das Objekt gemacht hat, hört es auf, primitive Kunst zu sein“ $(1991,156)$.

Man könnte meinen, dass, wenn es so schwierig erscheint, die Eigennamen afrikanischer Künstler ausfindig zu machen, dies daran liegt, dass in Afrika ein anderes Konzept von Kunst vorherrscht als dasjenige, das wir kennen, ja sogar, dass der Kunstbegriff dort keine Gültigkeit hat. Jack Goody bemerkt, dass wenn die Diskussion auf „afrikanische Kunst“ kam, „der Anthropologe Meyer Fortes beobachtete, dass die Tallensi, im Norden Ghanas, keine hatten“, ergänzt aber: „man kann den Gebrauch bezweifeln“ den Fortes vom Wort „Kunst“ macht; für ihn beschränkt sich Kunst auf repräsentative Formen: Skulptur, Theater, Mythen ... die Tallensi aber kennen Musik, mündliche Kunst und stellen Objekte von künstlerischer Qualität her (Goody 1997, 2f). Mir persönlich scheint, dass es keinen Grund zu glauben gibt, Afrika kenne keine Kunst. Es gibt auch nicht ein einziges homogenes Afrika. Wenn man Rechenschaft über das Funktionieren afrikanischer Produktion aus afrikanischer Sicht ablegen wollte, müsste man nicht nur den künstlerischen Modus anders aufbauen, sondern mehrere künstlerische Modi konstruieren und daher mehrere künstlerische Räume je nach Region, Ethnie oder betrachtetem Stamm.

Ich denke, dass diese Konstruktionen nur durch afrikanische Forscher vorgenommen werden könnten. Vielleicht würden diese Forscher letztendlich zeigen, dass es keine wirklichen Unterscheide zu unserem Kunstraum gibt, das zum Beispiel die Konzeption afrikanischer Kunst ebenfalls einen Eigennamen verlangt (warum sollten afrikanische Gesellschaften ohne Urheber sein?) und dass es nur an unserem Mangel an Informationen liegt, dass wir das Gefühl von Anonymität haben oder dass es in mündlichen Kulturen die Tendenz gibt, bedingt durch die Natur der mündlichen Verständigung, dass sich die individuelle Handschrift allmählich auflöst (wie Goody 1977 in The Domestication of the Savage Mind schreibt), aber das kann man nicht von vornherein voraussetzen. 
Definition des künstlerischen Modus (vollständige Form)

Vom Eigennamen zum Objekt:

- enunziative Ebene: Zuweisung eines Eigennamens;

- diskursive Ebene: Auffüllung des Eigennamens (biografische Recherchen, thematische und stilistische Analysen, Vergleiche mit anderen Künstlern, Kunstgeschichte);

- affektive Ebene: unbestimmt.

Vom Objekt zum Eigennamen:

- diskursive Ebene: thematische und stilistische Recherche, Vergleich mit anderen Produktionen, Gruppen- und Untergruppenbildung, biografische Recherchen;

- enunziative Ebene: Recherche und Zuweisung eines Eigennamens;

- affektive Ebene: unbestimmt.

\section{Beziehungen zwischen den Modi und zwischen den Räumen}

Wir haben gesehen, dass die Trennung des künstlerischen vom ästhetischen Modus einige Verhaltensweisen $\mathrm{zu}$ verstehen erlaubt, die andernfalls ungeklärt blieben. Ein weiterer Vorteil dieser Trennung liegt darin, dass man sich Fragen zur Beziehung zwischen den zwei Modi stellen kann, sowie zur Beziehung mit den Räumen, denen sie angehören. Diese Befragung lässt sich in vier Fragen aufteilen.

1. Führt die Zugehörigkeit zum künstlerischen Raum und die Inanspruchnahme des künstlerischen Modus zum ästhetischen Modus?

Schon indem man diese Beziehung in Frage stellt, vollzieht man offenbart einen Bruch mit der Doxa, die verlangt, dass „die ästhetische Erfahrung ein intrinsisches Ziel und Wert [oder auch eine ,aus- 
reichende Rechtfertigung، ${ }^{3}$ ] der Kunst konstituiert“, der oben zitierten Formulierung von Richard Shusterman folgend. Allerdings: Dass sie nicht obligatorisch ist, heißt nicht, dass sie sich nicht ergeben kann. Mir ist es etwa mit Stéphane Mallarmé so ergangen. Er hat mich immer gelangweilt, obwohl ich wusste, dass Mallarmé ein großer Dichter ist, der fraglos dem Raum der Kunst angehört (künstlerische Zuschreibung). Es verhielt sich aber so, dass Mallarmé in das Lernprogramm des CAPES ${ }^{4}$ aufgenommen wurde und ich daher verpflichtet war, sein Werk ausführlich durchzuarbeiten, um es an meine Studierenden heranzutragen. Durch das Lesen und Wiederlesen seiner Texte, durch das Anfertigen stilistischer Analysen seiner Gedichte (= Mobilisierung des vollständigen künstlerischen Modus), bin ich dazu gekommen, in ihnen einen bis dahin ungeahnten Reichtum zu entdecken, mich zu einer Suche nach Werten aufzumachen und so zu einer Lektüre im ästhetischen Modus überzugehen, einer Lektüre, die wiederum von meiner Zugehörigkeit $\mathrm{zu}$ einem bestimmten ästhetischen Raum bestimmt war (entsprechend meiner Stellung in der Gesellschaft).

2. Kann der ästhetische Modus zum künstlerischen Modus und zum Raum der Kunst führen?

Sogar wenn nicht jeder Zugang zum Raum der Kunst aus einer ästhetischen Herangehensweise resultiert - die ökonomischen Interessen sind oft ein starker Antrieb, wie man beim Kino sehen konnte -, die Möglichkeit besteht: Weil dieses Werk mich berührt hat, weil es mich zu einer bereichernden ästhetischen Erfahrung verleitet hat, möchte ich es in den Raum der Kunst überführen, damit es eine institutionelle und soziale Anerkennung erhält, die es ohne diese Zuschreibung nicht erhalten hätte. Die Arbeit besteht dann darin, institutionelle Unterstützung $\mathrm{zu}$ finden: Kritiker, Kunsthändler, Museumsverantwortliche, Kunsthistoriker, die den Eingang dieser

3 [Anm. d.Ü.:] So heißt es im Original und in der französischen Übersetzung, aus der Odin zitiert.

4 [Anm. d. Ü.] Dabei handelt es sich um das Diplom zur Lehrbefähigung in der Mittel- und Oberstufe. 
Produktion in den Raum der Kunst durch die Anwendung des künstlerischen Modus legitimieren.

Der Einsatz von Jean Dubuffet zu Gunsten der Produktionen von Geisteskranken und Randgruppen ist beispielhaft für diese Vorgehensweise. Dubuffet interessiert sich für „Werke, die frei von künstlerischer Kultur sind, in denen die Nachahmung, im Gegensatz zu dem was bei Intellektuellen vorgeht, wenig oder keinen Anteil hat, so dass deren Urheber alles aus ihrem eigenen Erfahrungsraum schöpfen (Themen, Auswahl der Materialien, Umsetzungsmittel, Rhythmus, Schreibweisen, etc.) und nicht aus Gemeinplätzen der klassischen oder zeitgenössischen Kunst“ (Trevoz 1995, 11). Zwar verwendet er den Ausdruck Kunst in seinen Schriften, aber was Dubuffet wirklich begeistert, sind die in den Produktionen verhandelten Werte (wir befinden uns im ästhetischen Modus): „Wir wohnen einem reinen, rohen künstlerischen Geschehen bei, von seinem Urheber in allen Phasen vollkommen neu erfunden, ausgehend allein von seinem eigenen Antrieb. Kunst also, in der sich einzig eine erfinderische Funktion manifestiert und nicht die ständige chamäleon- oder affenhafte der Kunstkultur“ (Dubuffet, zit. n. Trevoz 1995, 11). Um seine Werte zu verteidigen, legitimiert Dubuffet diese Produktionen durch einen Rückgriff auf den künstlerischen Modus, das heißt, in dem er alles daran setzt, die Eigennamen der Urheber zu Tage zu bringen: Förderung der Künstler (wie etwa Wôlfi, Aloïse oder Müller), Etablierung von Angaben zu ihrer Arbeit und ihrer Biografie, Werkanalysen, Gründung einer Fachzeitschrift (L'Art brut, erste Ausgabe: 1964) und sogar eines Museums (da es ihm nicht gelang, es in Frankreich zu bauen, errichtete Dubuffet es 1976 in Lausanne; vgl. Peiry 1999 [1997]). Das Endergebnis bedeutet nicht weniger als die Schaffung eines neuen Kunstraums: die Art brut - Dubuffet konnte sogar als „Erfinder der Art brut“ angesehen werden (Trevoz 1995, 11) -, eines Kunstraums, der sich vom Raum der „Kunstkulturen“ unterscheidet.

Diese Analyse bestätigt die Hypothese zur Situation der Kunst in Afrika, wonach zu einem Zeitpunkt in der Geschichte (synchron) und in ein und demselben Kulturraum mehrere Kunsträume existieren können. Es ist interessant festzustellen, dass die Prozesse des künstle- 
rischen Modus, die man in beiden Kunsträumen vorfindet (Raum der Kulturkunst vs. Raum der Art brut), dieselben sind. Was sich ändert, wenn man vom Raum der Kulturkunst in den Raum der Art brut wechselt, sind (einzig und allein) die über den ästhetischen Modus vermittelten Werte, der zur Mobilisierung des künstlerischen Modus geführt hat.

3. Kann die Zugehörigkeit zum Kunstraum die Mobilisierung des ästhetischen Modus blockieren?

Es kann vorkommen, dass das Erkennen der Zugehörigkeit zum Raum der Kunst den ästhetischen Modus blockiert. Die Einordnung des Theaters als Kunst kann als Grund für die Blockade einer ästhetischen Annäherung durch die Arbeiterklasse angesehen werden, im Gegensatz zum Kino. Ein ähnliches Problem existiert mit dem gehobenen Ort der Kunst, den der Raum des Museums darstellt. Was hier zur Debatte steht, kann man als „Arroganz der Kunst“ bezeichnen (Hamashita 2009), die die Entfaltung des ästhetischen Modus verhindert: Ich werde erdrückt vom Gewicht einer Zuschreibung, eine Zuschreibung, die außerdem als Klassenzugehörigkeitszeichen gelesen werden kann (Bourdieu). Das Ergebnis ist, dass ich keine Lust mehr habe, mich weiter damit zu beschäftigen, sondern mich mit dem Erkennen dieser Zuschreibung begnüge.

4. Kann der ästhetische Modus den Eingang in den Raum der Kunst blockieren?

Der ästhetische Modus kann seinerseits den Eingang in den Raum der Kunst blockieren: Es gab viele Dichter, Maler, Filmemacher, die zumindest eine Zeit lang vom Raum der Kunst ausgeschlossen wurden, weil die Werte, die das Ziel ihrer ästhetischen Suche ausmachten, nicht denen ihrer Zeit oder ihres Landes und damit dem dominanten Kunstraum zu jenem historischen Zeitpunkt entsprachen.

Von den Impressionisten (das ist keine Malerei) zur konkreten Musik (das ist keine Musik, das ist Krach) über das experimentelle Kino (das ist kein Kino): Es existieren viele Beispiele für Werke, die man 
vom Raum der Kunst fernzuhalten versucht hat und denen man die Nennung der Urheberschaft verweigert hat (die Berücksichtigung des Eigennamens). Das war auch der Fall bei Werken der Art brut vor dem Eingreifen Dubuffets.

Die Entscheidung, die beiden Modi zu trennen, erweist sich also als produktiv. Ein weiteres Mal sind es die durch die Analyse aufgeworfenen Fragen, die am interessantesten erscheinen: die Frage nach der Beziehung zwischen textueller Produktion und sinnlichen Beziehungen, die Frage nach der Beziehung zwischen den Modi und vor allem die Frage nach der Beziehung zwischen den Modi und den Kommunikationsräumen.

Im Prinzip lassen sich die Modi in einem gegebenen Kommunikationsraum sehr flexibel anwenden: Jeder beliebige Modus kann quasi in jedem beliebigen Raum auftreten. In der Praxis jedoch wird sich der Rezipient, wenn er will, dass die Kommunikation funktioniert (was keine Pflicht ist), bemühen, dem Kommunikationsraum zu folgen, den der Sender für ihn vorgesehen hat (wenn er denn über die diesbezügliche Information verfügt) und wird dem Modus oder den Modi der Sinnproduktion Vorrang gewähren, die dieser Raum verlangt. Dies blockiert zwar nicht die Anwendung anderer Modi, diese treten jedoch nur an zweiter Stelle auf, oft punktuell und unvorhersehbar (der Forscher hat also in der Regel keine Möglichkeit, sie einzubeziehen). Die Dinge werden noch komplizierter, wenn eine Produktion in einen Kommunikationsraum übergeht, der nicht der ihre ist, denn dieser Raum wird versuchen, seinen eigenen Modus durchzusetzen. In diesem Fall muss man die Beziehung zwischen dem ursprünglichen Modus und dem im neuen Kommunikationsraum verlangten Modus hinterfragen. Auf diese Fragen komme ich in den folgenden Kapiteln zurück. 



\section{Kapitel 4}

\section{Kontextuelle Analyse und Kommunikationsraum \\ Der Kommunikationsraum des Familiengedächtnisses}

In diesem Kapitel werde ich zeigen, wie der Begriff des Kommunikationsraums verwendet werden kann, um zu verstehen, was aus kommunikativer Sicht in einem bestimmten Kontext geschieht.

Als Beispiel dient mir die Kommunikation in der Institution Familie, wie sie in der westlichen Welt existiert. Aber die Untersuchung der Familie aus kommunikativer Sicht kann vieles bedeuten. Zunächst muss ich mich entscheiden, die Relevanzachse festzulegen, um den Kommunikationsraum zu konstruieren, in dem ich arbeiten werde. Ich habe die Qual der Wahl: zwischenmenschliche Beziehungen, Paarbeziehungen, Eltern-Kind-Kommunikation, die Beziehung $\mathrm{zu}$ den Medien, der Einfluss der räumlichen Organisation des Hauses oder der Wohnung auf die Familienkommunikation usw. Ich habe mich dafür entschieden, mein Interesse auf den Kommunikationsraum des Familiengedächtnisses $^{1} \mathrm{zu}$ legen, den ich für eine wichtige Achse halte: Durch die Schaffung von Verbindungen zwischen der Gegenwart und der Vergangenheit ist das Gedächtnis das, was die innere Beständigkeit der Familie gewährleistet. Dass ich alles, was nicht zu dieser Achse gehört, von der Untersuchung ausschließe, heißt nicht, dass ich alles, was diese Achse betrifft, untersuchen kann (wichtige Fragen, wie zum

1 [Anm. d. Ü.:] Der Begriff „mémoire“, den Odin in diesem Kapitel verwendet, lässt sich sowohl mit Erinnerung als auch mit Gedächtnis übersetzen und nivelliert also die konzeptuelle Differenz zwischen aktiver Erinnerung und passivem Gedächtnis, die im deutschsprachigen Diskurs etabliert ist. Der Begriff wird bei Odin häufig in beide Richtungen deutbar verwendet - hier wird er in der Regel mit Gedächtnis übersetzt. 
Beispiel die leidenschaftliche Stammbaumforschung vieler Familien, werden nicht behandelt). Auch hier besteht mein Ziel mehr darin, eine Methode zu veranschaulichen, als diesen oder jenen Kommunikationsraum im Detail zu analysieren.

\section{Der Kommunikationsraum des Gedächtnisses in der „traditionellen“ Familie}

Mit „traditioneller“ Familie meine ich die bürgerlich-patriarchale Familie, wie sie in den Jahren 1945-1975, der großen Zeit des Familientums, vorherrschte. Diese Struktur kann als eine Reihe von Bedingungen beschrieben werden, die die Konstruktion der Aktanten dieses Raumes steuern.

Die Bedingungen und die Konstruktion der Aktanten

„Ganz allgemein gesprochen weist jede Gruppe Plätze zu. Diese behält man im oder formt sie zum Gedächtnis“ (Ricœur 2004 [2000], 188), bemerkt Paul Ricœur in seiner Reflexion über Maurice Halbwachs. Laut Halbwachs ist dieses System von Plätzen innerhalb der Familie besonders stark und stabil:

Die Menschen können von einem Beruf $\mathrm{zu}$ einem anderen wechseln, von einer Staatsangehörigkeit $\mathrm{zu}$ einer anderen übergehen, sie können in der sozialen Hierarchie auf- oder absteigen, Untergebene können Chefs werden und Chefs Untergebene, ein Laie kann sogar Priester werden und ein Priester wiederum Laie. Aber ein Sohn wird nur dann Vater werden, wenn er eine andere Familie gründet, und selbst dann wird er stets der Sohn seines Vaters bleiben; es besteht da ein unumkehrbares Verhältnis. Und ebenso können Brüder nicht aufhören, Brüder zu sein; hier besteht eine unauflösliche Einheit. Nirgends scheint die Stellung des Individuums mehr vorausbestimmt zu sein, ohne daß auf seinen Willen und auf das, was es ist, Rücksicht genommen wird. (Halbwachs 1985 [1925], 223f) 
Seit Freud ist auch bekannt, dass diese Bestimmungen nicht nur die biologischen Verwandtschaftsbeziehungen betreffen, sondern ebenso die symbolischen Verortungen, die sich in der ödipalen Beziehung manifestieren.

In dieser Struktur hat der Vater einen besonderen Status. Es ist daher nicht verwunderlich, dass er derjenige ist, der die Konstruktion des Familiengedächtnisses vorantreibt (er ist derjenige, der das Grab errichtet, der die gemalten Porträts bestellt, der die Fotos macht, der filmt ...), aber dies geschieht, indem er die ganze Familie einbezieht. Besorgt um die Kontinuität der Generationen, organisiert er große Erinnerungsrituale: anlässlich von Geburten, von Übergangsriten (Erstkommunion, Ehe, Ruhestand), von physiologischen Veränderungen (der erste Zahn, die ersten Worte, die ersten Schritte), von materiellen Veränderungen (ein neues Haus, ein neues Auto ...). Er sorgt für Besuche auf dem Friedhof, lädt zu großen Mahlzeiten ein, projiziert die Familienfilme ... Er ist es, der die (Re)Konstruktion der Familiengeschichte durch die Familienmitglieder überwacht, eine mehr oder weniger mythische Geschichte, die äußerlich als offizielle Geschichte und innerlich als Konsensstifter fungiert oder zumindest der Erzeugung eines scheinbaren Konsenses dient. Auf dieser Ebene ist die Familie (die Familie als Struktur) der wahre Enunziator der Gedächtnisarbeit: Die Institution bemüht sich um ihre Erhaltung und sorgt dafür, dass nichts ihre Harmonie stört. Begleitet wird die väterliche Zensur von einer Selbstzensur: Es gibt Dinge, über die man nicht spricht.

Aber die Familie ist nicht nur eine Struktur, sondern auch eine Gruppe von Individuen, und wir können die Beziehung zum Familiengedächtnis nicht begreifen, ohne diesen doppelten Status zu berücksichtigen. Selbst Halbwachs, der, wie wir wissen, die These verteidigt, dass individuelle Erinnerungen immer auf sozialen Beziehungen und damit auf dem Kollektiv beruhen, macht diese Unterscheidung:

So existiert kein Ereignis oder Gesicht im Gedächtnis der Familie, das nicht diese beiden Eigenschaften aufwiese: einerseits gibt es ein einzigartig reichhaltiges und tiefes Bild, da wir darin die Wirk- 
lichkeit wiederfinden, die wir persönlich aus intimster Erfahrung kennen; andererseits müssen wir es vom Standpunkt unserer Gruppe betrachten, d.h. uns der Verwandtschaftsbeziehungen erinnern, die erklären, warum es für alle unsere Angehörigen von Interesse ist. (Ibid., 225)

Es gibt also, mehr unter der Oberfläche, ein anderes, zutiefst individuelles Familiengedächtnis. Dieses Gedächtnis ist nicht freier als das vorherige, aber die Bedingungen, die es regieren, betreffen nicht die Gruppe, sondern die Person selbst. Dieses verborgene Gedächtnis, das im Inneren jedes Menschen wirkt, führt zu einer Textproduktion, die sich sehr von der obigen unterscheidet, eine Produktion in oft dysphorischer Tonlage: Hier zeigen sich die alten Missstände, die alten Konflikte zwischen Familienmitgliedern, all das Unausgesprochene, das die Schattenseite der Familie bildet.

Welche(n) Modus/Modi konstruieren?

Privater Modus, intimer Modus

Die Existenz dieser beiden Gedächtnisse hat zur Folge, dass man nicht, wie der Vertreter der visuellen Anthropologie Richard Chalfen mit dem „Home mode“ (Chalfen 1987), an der Konstruktion eines einzigen Modus festhalten kann. Zwei Modi müssen entsprechend den beiden identifizierten Erinnerungstypen konstruiert werden: der private Modus und der intime Modus.

Mit privater Modus meine ich den Modus, in dem eine Gruppe (hier die Familie) in ihre Vergangenheit zurückkehrt. Mit dem privaten Modus befinden wir uns in dem, was Edward S. Casey Reminiscing nennt: die Vergangenheit zu beleben, indem man sie kollektiv zum Beispiel durch die Konversation hervorruft, aber auch durch das Teilen von Fotos oder Videos (Casey 2000: 104-121). Hier wird die Kommunikation veräußerlicht (sei es durch Sprache, Ton oder Bild) und unterliegt daher direkt den Bedingungen (der Zensur) der Familienstruktur. Das hat zur Folge, dass die in diesem Modus hervorgebrachten Ereignisse stark standardisiert sind (es gibt nichts, was einem Familienfilm so ähnlich sieht wie ein Familienfilm) und 
meistens einen euphorischen Ton haben. Eine andere Konsequenz ist, dass das Gesagte (die Textproduktion) in diesem Fall oft weniger wichtig ist als das Sagen selbst: Das Wesentliche liegt im Austausch zwischen den an der Kommunikation beteiligten Aktanten.

Mit intimer Modus meine ich den Modus, mit dem ich zu meinem Leben und der Vergangenheit der Familie zurückkehre. Der intime Modus manifestiert sich in Form eines innerlichen Diskurses: Es gibt keine Veräußerung der Kommunikation. Es ist wohl kaum nötig, auf die Kraft der Affekte zu insistieren, die diese innere Produktion und ihre Rolle in der Identitätskonstruktion des Individuums beleben, eine Konstruktion, die man macht, um sich von den Anderen abzugrenzen.

Wie die ästhetischen und künstlerischen Modi, haben der intime und der private Modus eine horizontale Funktion und sie lassen sich durch eine narrative Struktur beschreiben. Diese Struktur kann, abhängig von der Antwort auf folgende Fragen, unterschiedliche Szenarien hervorbringen: Was löst die Gedächtnisbewegung aus? Welche Art von Bewegung ist das? Wer sind die Gegner? Hier sind einige Beispiele.

Erzählung des Auftauchens: Ein Objekt drängt sich mir auf und löst die Erinnerung aus. Die Erinnerung ,entsteht in der Art einer Zuneigung“, sagt Ricœur (2000, 22): Dies ist das Szenario von Prousts Madeleine. Wir sind im intimen Modus. Es gibt keine Absichten in dieser Welle des Erinnerns, weder meinerseits noch seitens des Objekts, das nicht gemacht wurde, um die Erinnerung in Bewegung zu setzen. Hierbei ist das Objekt nicht nur ein Vehikel des Gedächtnisses, sondern ein Stimulator der Erinnerung [mémoire].

Erzählung der Suche: Sie manifestiert sich auf zwei Ebenen. Auf der intimen Ebene ist es das umgekehrte Szenario des vorherigen; ich gehe auf die Suche nach Familienerinnerungen. Dies ist die Strategie des Erinnerns (des In-Erinnerung-Rufens), die Ricœur als „aktive Suche“ (ibid., 22) definiert. Hier ist die Absicht auf meiner Seite (ich bin das Subjekt, das auf der Suche ist) und die Erinnerung ist das Objekt meiner Suche. Es kann passieren, dass diese Suche auf seinen Opponenten, das Vergessen, stößt. Ich muss dann auf Hilfsmittel zurückgreifen müssen, um meine Ziele zu erreichen: andere Familienmitglieder befragen, ins Archiv gehen, Fotos anschauen, Briefe wieder 
lesen usw. Auf privater Ebene hat die Suche eine kollektive Dimension: Die vereinigte Familie teilt die Suche nach der Erinnerung (Familienessen, Treffen am Familiengrab, das Vorführen der Familienfilme usw.).

Erzählung der Transformierung: X gibt einem Objekt Y absichtlich den Status eines Bewahrers des Familiengedächtnisses, so dass es ihn in der Zukunft an den Moment erinnert, in dem er lebt (intimer Modus). In La Photo sur la cheminée (1993) beschreibt Bertrand Mary ein junges Ehepaar, das unter einer Glasglocke eine große Silbermünze aufbewahrt, die vom Priester gesegnet wurde, zusammen mit den Trauringen, dem Orangenblütenkranz der Braut sowie dem weißen Band, das sie in ihren Haaren trug (privater Modus). Wir befinden uns in der Logik des „Talismans“ oder in dem, was Krysztof Pomian (über den Eintritt von Objekten in Museen) das Gebiet der Semiophoren nennt: Es geht darum, das Objekt in mein persönliches, privates Museum zu bringen (Pomian 1990). ${ }^{2}$

Erzählung der Schenkung: Nicht mehr bin ich es, sondern es ist eine andere Person, die mir absichtlich ein Objekt gibt, dem sie den Status eines Bewahrers der Erinnerung (privater Modus) verleiht: Es ist die Verlobte, die sich eine Haarlocke abschneidet, um sie ihrem Verlobten zu geben, der Vater, der seinem Sohn eine Uhr gibt, damit der sich an ihn erinnert. Auch hier zielt die Markierung des Gedächtnisses auf die Zukunft.

Erzählung der Übertragung: Mein Vater erzählt mir, wie sein Leben als Kind war. Ich erhalte einen Brief, in dem meine Tochter mir von ihrem Strandurlaub erzählt. Das Gespräch, der Brief (aber es könnte auch eine Reihe von Fotos, Tonaufnahmen, ein Video etc. sein) sind Erinnerungsvehikel: Sie übermitteln Gedächtnisinhalte, um gegen das Vergessen zu kämpfen. Die zwei Modi, intimer Modus und privater Modus, können sich ihrer dann annehmen.

Die Verwendung eines Modus verhindert nicht den gleichzeitigen Gebrauch des anderen: Man kann an der Erforschung des kollektiven Gedächtnisses (privater Modus) teilhaben, während man individuell in seine Vergangenheit zurückkehrt (intimer Modus) und umgekehrt.

2 [Anm. d.Ü.:]Bei Pomian bezeichnet Semiophor ein Objekt, dass durch seinen Einzug in die Sammlung eines Museums einen Bedeutungs-wandel erfährt; vgl. Pomian, Krysztof (1988): Der Ursprung des Museums: Vom Sammeln. Berlin: Wagenbach. 
Drei Haupttypen von Beziehungen können dann zwischen den beiden Modi hergestellt werden.

Konstruktion paralleler Erzählungen: Während wir einen Familienfilm über die letzte Reise nach Griechenland anschauen und die versammelte Familie ihre Erinnerungen wachruft (kollektive Konstruktion: privater Modus), beginne ich, an meinen vor einem Jahr verstorbenen Großvater zu denken, den ich vermisse (intimer Modus). Privater Modus und intimer Modus arbeiten hier getrennt voneinander.

Konstruktion einer einzigen Erzählung durch Artikulation der beiden Modi: privater und intimer Modus verstärken sich gegenseitig; ich vertiefe das kollektiv Gesagte, ohne diese Gedanken aussprechen zu wollen. Der intime Modus bereichert die Gedächtnisrelation.

Konstruktion divergierender Erzählungen: Die Bedingungen des Kommunikationsraums des Familiengedächtnisses implizieren, wie wir gesehen haben, dass das, was im privaten Modus ausgesprochen wird, zum Zusammenhalt der Familie beiträgt oder diesen zumindest nicht in Gefahr bringt. Unter diesen Umständen ist es üblich, dass meine innere Stimme (intimer Modus) nicht mit dem übereinstimmt, was gesagt wird, und sogar mit dem, was ich in meiner mündlichen Kommunikation mit anderen Mitgliedern der Familie äußere (privater Modus).

Schließlich implizieren beide Modi die Konstruktion eines, in Bezug auf seine Identität befragbaren, realen Enunziators:

- Enunziator ICH für den intimen Modus: Die individuelle Erinnerung ist beteiligt an der Bildung meiner persönlichen Identität;

- Enunziator die Gruppe für den privaten Modus (hier die Familie): Der kollektive Ansatz trägt dazu bei, die Identität der Familie als Gemeinschaft in Bezug auf ihre Mitglieder, in Bezug auf andere Familien und in Bezug auf andere Gruppen, in denen sich Familienmitglieder engagieren können, aufzubauen und zu bestätigen (Schule, Arbeit, Sportverein. . .). Selbstverständlich trägt auch der kollektive Vorgang zur Bildung meiner persönlichen Identität bei. 
Definition des intimen Modus

- diskursive Ebene: innerlicher Diskurs;

- affektive Ebene: extrem kraftvoll/mächtig;

- enunziative Ebene: Konstruktion eines realen Enunziators: ICH;

- relationale Ebene: Konstruktion der Identität.

Definition des privaten Modus

- auf der diskursiven Ebene: unbestimmt in seiner Form, aber in einem gegebenen Kontext ziemlich stark standardisiert;

- auf der affektiven Ebene: euphorische Affekte, Zugehörigkeitsgefühl zu einer Gemeinschaft;

- auf der enunziativen Ebene: Konstruktion eines realen, kollektiven Enunziators;

- auf der relationalen Ebene: Handlungen im Innern einer Gruppe.

Man beachte, dass zur Beschreibung dieser beiden Modi eine Ebene hinzugefügt werden musste, die bis dahin für andere Modi verwendet wurde: die relationale Ebene.

Die Kommunikationsoperatoren

Einen Kommunikationsoperator nenne ich alles, was in einem bestimmten Raum bei den gewählten Relevanzkriterien, von den Aktanten genutzt wird, um Kommunikation zu ermöglichen.

Hier sind Kommunikationsoperatoren Gedächtnisoperatoren. Auf der Ebene der Textproduktion fungieren sie als Vehikel und Stimulatoren des Gedächtnisses. Auf der einen Seite geben sie mir Informationen, die mir direkt in Erinnerung bleiben: Ich konnte mich nicht erinnern, dass meine Tante so schön war - dieses Porträt vergegenwärtigt es mir. Auf der anderen Seite führen sie durch eine Kettenreaktion zu einer Textproduktion, die manchmal vollständig von dem vom Operator erzeugten Text abweichen kann: Wenn ich ein Foto meiner Großeltern sehe, denke ich an meinen Jugendfreund Pierre, den ich seit mehr als zwanzig Jahren nicht mehr gesehen habe, dann an meine Ängste während meines ersten Schulanfangs in der Grund- 
schule - das Geheimnis der Assoziationen. Auf der relationalen Ebene greifen sie in die Beziehung zwischen den Familienmitgliedern, in die Beziehung der Familie zu anderen Gruppen und in die Beziehung jedes Mitglieds der Gruppe zu sich selbst ein (in allen drei Fällen befinden wir uns in der Identitätsbildung).

Eine rasche Typologie macht es möglich, zwischen unbeabsichtigten Operatoren (Prousts Madeleine), Operatoren, die aus der Absicht des Empfängers entstanden sind (ich beschließe nach Châtel-de-Neuvre zurückzukehren, dem Dorf, in dem meine Großeltern lebten, und erhebe also ein Dorf zum Operator meines Gedächtnisses) und Operatoren, die als solche bereits produziert werden: das Grab (an dem sich die Familie jedes Jahr zu Allerheiligen trifft), die Abenteuergeschichten aus dem Krieg 1914-1918, die mein Großvater mir erzählt hat, gemalte Porträts, Postkarten, Fotografien, Filme ..., zu unterscheiden.

Aber es reicht nicht, die Operatoren zu zählen und zu klassifizieren; es ist auch notwendig, ihre Natur in Frage zu stellen. Wir stellen schließlich fest, dass ein Operator nicht unbedingt das ist, was wir für einen solchen halten würden.

So werden im Kommunikationsraum des Familiengedächtnisses gemalte Portraits nicht wirklich wie Gemälde gelesen. Auch wenn der künstlerische Modus als ein aufwertendes Element agieren kann („es ist ein Paulin“, sagte Frau X stolz, ihren Gästen ihr Porträt zeigend, Paulin war ein unbekannter Maler in der Gegend von Lyon ...), herrscht die Beziehung zur gemalten Person (das Indexikalitätsverhältnis) vor: „Sie ist es, wie sie leibt und lebt“. Tatsächlich werden diese Porträts wie Fotografien angeschaut, noch bevor das Foto existierte: Sie reklamieren für sich eine Lektüre, und auch auf Seiten des Senders eine Herstellung, im Modus des Abdrucks (mit jener Besonderheit, dass der Abdruck hier von Menschenhand gemacht wird, mediatisiert durch den Blick und die Arbeit des Malers). Ich bezeichne diesen Gedächtnisoperator als fotografische Malerei.

Wir könnten sodann auf einen Ansatz wie den der Remediatisierung zurückgreifen - „Jedes neue Medium ist dadurch gerechtfertigt, dass es einen Mangel ausgleicht oder einen Fehler seines Vorgängers behebt“ (Bolter/Grusin 2000) - und denken, dass der fotografische Operator das gemalte Porträt ersetzen wird, da die 
Fotografie direkt durch den indexikalischen Modus wirkt. („Die Herstellung des Abdrucks ist ein autonomer Vorgang, der nicht notwendigerweise durch ein menschliches Auge vermittelt wird.“ [Schaeffer 1987, 22]) Die Dinge sind jedoch nicht so einfach, denn die Fotografie scheint oft zu genau, zu präzise und, um es kurz zu sagen, $\mathrm{zu}$ wahrheitsgetreu $\mathrm{zu}$ sein; das Modell ist möglicherweise nicht ausreichend wertgeschätzt worden. Die Malerei feiert also mit der Retusche ein Comeback: eine zu große Nase wird verkleinert, einen fehlender Zahn ergänzt, ein entferntes Ohr nachgezeichnet ... Wir können somit von einem Operator der gemalten Fotografie sprechen.

Was als Operator für den Kommunikationsraum des Familiengedächtnisses geeignet ist, ist folglich ein neues Medium, ein Medium, das weder Malerei noch Fotografie ist, sondern eine Mischung aus beidem: Wir befinden uns hier nicht bei der Konvergenz (Jenkins 2006), sondern bei der Hybridisierung.

Dasselbe Phänomen der Hybridisierung findet sich beim Familienfilm wieder. Entgegen dem, was seine Bezeichnung vorauszusetzen scheint, ist der Familienfilm kein Film oder genauer: Er muss kein „Film“ sein, zumindest wenn „Film“ eine strukturierte Produktion zu Kommunikationszwecken mit einem Anfang und einem Ende bezeichnet. Wenn der Familienfilm wie ein Film aufgebaut ist, zeigt er eine Geschichte aus der Familie aus der Sicht desjenigen, der den Film gemacht hat (in der Regel der Vater) - eine Perspektive, die von den anderen Familienmitgliedern nicht akzeptiert werden muss: Sie werden es schwer haben, dort ihre eigenen Erfahrungen wiederzufinden. Um gut zu funktionieren, muss der Familienfilm als eine unorganisierte Serie von Aufnahmen komponiert werden, die nur Teile des Familienlebens offenbaren, so dass jedes Mitglied die Familiengeschichte auf seine eigene Weise rekonstruieren kann. Kurz gesagt, es muss weniger ein Vehikel als ein Stimulator des Gedächtnisses sein oder anders gesagt, der Familienfilm funktioniert gut, wenn er „schlecht gemacht“ ist (Odin 1995; 1999). Es ist daher falsch, zu denken, dass Amateur-Familienfilmemacher schlechte Filmemacher sind: Sie passen sich nur der Bedingung des Kommunikationsraums an, in den sie eingreifen. Diese Bedingung drückt sich folgendermaßen 
aus: Wenn man einen Familienfilm macht, verhält man sich nicht wie ein Filmemacher.

Wenn man umgekehrt einen Familienfilm „als Filmemacher" macht, dann wird dieser $\mathrm{zu}$ einer Quelle des Konflikts mit den Familienmitgliedern (der Konflikt ist die Sanktion, die die Verletzung der Bedingungen des Kommunikationsraums auszeichnet). Krystof Kieslowskis Film AMATOR (1979) erzählt die Geschichte eines Familienfilmers, Filip Mosz, der nach und nach von dem Wunsch (dem Dämon) ergriffen wird, „Kino zu machen“ (er inszeniert seine Familie) und der aufgrund dieses Rollenwechsels Frau und Kind verliert. Obwohl seine Geschichte viel weniger dramatisch ist, bezeugt Steven Spielberg seinerseits die Konflikte, die durch seine Position als Filmemacher entstehen, wenn er seine Familie filmt:

Was mein Vater da filmte, war allerdings unvorstellbar langweilig. Zum Einschlafen. Also nahm ich die Sache in die Hand. Ich sprang aus dem Auto, rannte voraus und sorgte dafür, dass die Anfahrt ein bisschen spannender wirkte. Wie in einem Gangsterfilm. Im Laufe der Zeit fing ich an, unsere Camping-Trips zu inszenieren. Meine drei Schwestern waren von mir genervt, weil sie das Gepäck mehrmals auspacken mussten. Aber sie sind unerbittlich. Sie wissen genau, was ich draußen gelassen habe. Und sie wollen, dass es wieder hineingeschnitten wird. Aber ich habe die künstlerische Kontrolle. Ich bleibe hart. (Spielberg 2004)

Ein weiteres Beweisstück auf der Rezeptionsebene: Wenn man sich einen Familienfilm als „Film“ ansieht, kann einem nur langweilig werden. Anders als der „Kinofilm“ ist der Familienfilm nicht dazu gedacht, den Zuschauern eine Geschichte zu erzählen, sondern nur das Familiengedächtnis in Bewegung zu setzen.

Aber wenn ein Familienfilm kein „Film“ ist, was ist er dann? Die Antwort scheint mir folgende zu sein: Der Familienfilm ist ein Album animierter Fotografien. Drei Argumente können diese Antwort unterstützen. Das ästhetisch-inhaltliche Argument: Der Familienfilm zeigt die gleichen Familienereignisse und verwendet die gleichen Stilfiguren wie die Familienfotografie (Pause, Frontalansicht, Kamerablick, Grup- 
penfoto ...), aber animiert. Das ethno-methodologische Argument: Wer einen Familienfilm macht, sieht sich nicht als „Filmemacher“, sondern als Fotograf. Hervé Guibert zitiert nach einer Vorführung von Familienfilmen eine Bemerkung seines Vaters: „Du musst enttäuscht sein, sagt mir mein Vater, das sind nur animierte Familienfotos“ (Guibert 1981, 51). Mit dem Familienfilm sind wir im fotografischen Abschnitt [strip] und nicht im kinematografischen Abschnitt (Abschnitt: „Ausschnitt aus einem in Gang befindlichen Vorgang [...] vom Standpunkt derjenigen aus gesehen, die subjektiv an den Dingen interessiert sind“ [Goffman 1980 (1974), 19]). Das strukturelle Argument: Der Familienfilm zeigt eine Folge von Lebensmomenten, die durch unterschiedlich große zeitliche Lücken (von einigen Minuten bis zu mehreren Tagen oder sogar mehreren Monaten) getrennt sind; diese Momente haben oft nichts miteinander zu tun, außer dass sie zur Familiengeschichte gehören; wir befinden uns in der chronologischen Folge, nicht in der Narration. Das ist genau die Struktur des Familienalbums.

Was diese beiden Beispiele (Familienporträt und -film) zeigen, ist, dass, um die in einen Kommunikationsraum eingreifenden Operatoren $\mathrm{zu}$ analysieren, das Festhalten an den bereits bestehenden Medien nicht ausreicht. Es ist notwendig zu analysieren, wie jeder Kommunikationsraum seine Operatoren konstruiert. Diese Konstruktion zielt darauf ab, den Operator an seine Funktion im betrachteten Kommunikationsraum anzupassen. Zum Beispiel ist das Interesse, den Familienfilm als „Familienfotoalbum“ (= mit einer „lückenhaften“ Konstruktion) zu strukturieren, ein doppeltes: Nicht nur erlaubt diese Konstruktion eine individuelle Beziehung jedes Familienmitglieds mit der Familiengeschichte (jeder kann seine Erfahrung finden, da keine Erzählung aufgezwungen wird), aber sie drängt auf eine kollektive Rekonstruktion dieser Geschichte durch alle Mitglieder der Familie, da es notwendig ist, die Lücken zu füllen; und es ist wahr, dass während der Vorführung eines Familienfilms viel geredet wird, so dass wir oft aufhören, den Film zu schauen. Der Familienfilm (wie das Fotoalbum) funktioniert eher wie ein relationaler Operator als ein textueller Operator. 
Fassen wir zusammen: Die in der Familie herrschenden Bedingungen regeln den Aufbau der Operatoren, der Aktanten und der Modi, so dass die Gedächtniskommunikation „bestenfalls“ zwischen den Familienmitgliedern und zugunsten der familiären Institution erfolgt.

Insbesondere das System der Zensur/Selbstzensur, das in die Kommunikation zwischen den Familienmitgliedern eingreift, und die Trennung zwischen zwei Diskursmodi, einer extern, normalisiert und euphorisch (der private Modus), der andere intern (der intime Modus), der den Ausdruck von Problemen auf das Ungesagte verweist und gleichzeitig ihre Formulierung für den Einzelnen zulässt, bilden zwei Hauptfaktoren dieses ,guten‘ Funktionierens. Wenn der intime Diskurs externalisiert wird, wenn die Zensur nicht respektiert wird, wenn die Operatoren nicht so konstruiert werden, wie sie ,sein müssen', werden diese Übertretungen sanktioniert: Das wird Konflikte in der Familie schaffen. So gesehen, scheint das Funktionieren der Gedächtniskommunikation in der Familie vor allem eine ideologische Funktion zu haben: den Zusammenhalt der Familie zu fördern, das Familientum zu stärken und die Institution in ihrer traditionellen Form zu erhalten.

\section{Der Kommunikationsraum des Gedächtnisses in der neuen Familienstruktur}

Halbwachs weist darauf hin, dass einige Familien, „da sie für die gegenwärtigen Bedingungen mehr Sinn hatten als für den Respekt vor dem Vergangenen“, ihr Leben auf neuen Grundlagen organisiert haben.

Sie bilden die Züge einer Gesellschaft vor, in der die von den besonderen Traditionen zwischen den Familiengruppen aufgerichteten Schranken niedergerissen sind, das Familienleben nicht mehr den einzelnen gänzlich absorbiert und die Familie sich ausweitete und teilweise mit anderen Gruppenformen verschmelzen würde. Ihre Ideen und Überzeugungen stellen die gerade entstehenden Traditionen dieser weiteren Gruppen dar, in denen die alten Familien absorbiert werden. (Halbwachs 1985 [1925], 385) 
Diese Beschreibung von Halbwachs gibt einen ziemlich guten Überblick darüber, wie also Aktanten in der neuen Familienstruktur konstruiert werden: Wenn hierarchische Bedingungen abnehmen, haben Individuen Vorrang vor der Institution, und persönliche Beziehungen haben Vorrang vor Verwandtschaftsbeziehungen (dies nennt man gelegentlich „Wahlverwandtschaften“).

Auf der anderen Seite hat, parallel zur Entwicklung der Familie, eine beispiellose technologische Entwicklung die Gedächtnisoperatoren des Familienraums sowohl auf der Ebene der Produktion als auch auf der Ebene der Vermittlung direkt beeinflusst: Das Aufkommen des Fernsehens, das sich als sehr guter Lehrer der audiovisuellen Sprache erwiesen hat - jetzt „weiß“ jeder zu filmen und sogar zu schneiden: die Codes wurden integriert - vom Filmstreifen über das Video bis hin zum Digitalen, die Möglichkeit der direkten Tonaufnahme, das Aufkommen von Apparaten zur Bild- und Tonaufnahme in jeder Größe, die Verbreitung des Mobiltelefons und des Computers, die Entwicklung des Internets. Zwar muss man sich davor hüten, in technologischen Determinismus zu verfallen, aber es ist sicher, dass diese Entwicklungen die Bedingungen ändern; auch werden wir sehen, dass sie neuen Familienstruktur angemessene Werkzeuge liefern.

Diese Veränderung des institutionellen und technologischen Kontexts impliziert die Änderung der Charakterisierung des Kommunikationsraums des Familiengedächtnisses, wie er gerade vorgestellt wurde. Der erste Gedanke, der einem zu Bewusstsein kommt, ist der, dass diese Evolution als Transformation des vorherigen Raums $\mathrm{zu}$ betrachten ist. Es ist jedoch nicht diese Lösung, die ich in Betracht ziehen werde: Aus einem deskriptiven Blickwinkel scheint es wirksamer zu sein, einen neuen Kommunikationsraum zu konstruieren, einen anderen Raum, vom vorhergegangenen unterschieden auf der Ebene der Operatoren ebenso wie auf der der Sinnproduktion und Textproduktionen. Einerseits weil sich die Dinge in Bezug auf die Erfahrung in diesem neuen Raum ziemlich drastisch ändern, andererseits weil dieser neue Raum den vorherigen nicht verschwinden lässt. In unterschiedlichen Graden ist die aktuelle Familie eine gemischte Struktur, die zwischen den beiden Räumen zögert: Es gibt also einen 
Schnittpunkt zwischen den beiden Räumen. Wenngleich die folgenden Analysen vor allem darauf abzielen, den neuen Raum zu charakterisieren, werde ich auf einige Eigenschaften hinweisen, die von der Beständigkeit des vorherigen Raums zeugen.

Eine „befreite“ Kommunikation

Die Folge der Reduktion institutioneller Bedingungen innerhalb der Familie ist, dass die neuen Gedächtnisproduktionen nicht zögern, Aspekte des Familienlebens zu offenbaren, die bis dahin unausgesprochenen geblieben sind.

Wir brechen mit dem euphorischen Blickwinkel der Familienbilder, wir werfen einen Blick auf die Familie „wie sie ist“, mit ihren sicherlich glücklichen Momenten, aber auch mit all ihrer kleinen Schäbigkeit und all den Momenten der Rivalitäten und Konflikte, die nicht ausbleiben, sich in einer jeden Gruppe zu produzieren. Die Einführung des Direkttons begünstigt diese Bewegung: Da der Ton viel schwieriger zu kontrollieren ist als das Bild - insbesondere alles, was im hors-champ geäußert wird -, werden in der neuen Produktionen Dinge gesagt, die zuvor undenkbar waren: Wörter, die man lieber vergessen hätte, unangenehme Bemerkungen, Dementis, die schlimmer sind als alle Behauptungen, angedeutete Attentäter usw.

Aber gerade die Beziehung zur Intimität hat sich verändert. Die Veränderung der institutionellen Bedingungen sowie die technologische Entwicklung bedeuten, dass der Aufnahme nichts mehr entgeht. In FAMILY VIEWING (Atom Egoyan, CAN 1987) entdeckt ein Sohn, dass sein Vater Familienfilme löscht, um sich beim Sex mit seiner neuen Partnerin zu filmen. Das Sich-Selbst-Filmen entwickelt sich. In einer Werbung hält eine Frau, die auf dem Rücken liegt, ihre Videokamera, die sie auf sich richtet. Der Slogan lautet: „Es ist mein Film, es ist mein Leben.“ Meines Wissens hat keine Werbung für das Amateurkino die Möglichkeit des Sich-Selbst-Filmens als Verkaufsargument genutzt. Mit kleinen Kameras, insbesondere mit dem Handy, wird noch ein weiterer Schritt getan. Diese Geräte stellen mit ihrem Besitzer eine echte Intimitätsbeziehung her (wir haben sie immer in der Tasche, wir halten sie in der Handfläche): das Sich-Selbst-Filmen wird zu einem 
allgemeinen Vorgang (man vertraut sich seinem Handy an, wie man sich seinem Kuscheltier anvertraut).

Ein anderer Modus der Sinnproduktion:

Der Modus der Zeugenschaft

Heute ist es nicht mehr allein der Vater, der die Familienfotos und -filme aufnimmt, sondern alle Familienmitglieder. Die Bereitstellung von einfach zu bedienenden Geräten macht dies möglich. Wir beobachten dann eine Vielzahl von Produktionen, die aus verschiedenen Blickwinkeln bestehen: die Sichtweise des Vaters, die der Frau, aber auch die der Kinder, die ihr eigenes Gerät haben (oft ein Mobiltelefon). Eine individuelle enunziative Struktur ersetzt die kollektive enunziative Struktur (die Familie). In der neuen Familienstruktur werden das Familienfotoalbum und der Familienfilm einer Vielzahl von Fotos und Filmen über die Familie zugänglich gemacht.

Ein neuer Modus der Sinnproduktion greift ein, ein Modus, der den privaten Modus und den intimen Modus ergänzt und nicht ersetzt: der Modus der Zeugenschaft.

Mit dem Modus der Zeugenschaft meine ich ein Subjekt, ein ICH, das durch die Herstellung eines Textes (geschrieben, mündlich, Bild und/ oder Ton) seine Ansicht zu dem, was es sieht oder was es gesehen hat, wiedergibt; hier, das Familienleben.

Diese Konstruktion eines Enunziators ICH unterscheidet sich von jener beim intimen Modus dahingehend, dass der Enunziator sich nicht im innerlichen Diskurs ausdrückt, sondern durch einen manifesten Diskurs die anderen Mitglieder der Familie adressiert. Der Modus der Bezeugung hat also sowohl eine intime also auch eine kollektive Dimension. Ein anderer Unterschied zum intimen Modus ist folgender: Während wir für den intimen Modus auf das zurückgreifen können, was Käte Hamburger zum Lyrischen Ich sagt: „dann dürfen wir nicht mehr feststellen, ob der Aussageinhalt wahr oder falsch, objektiv wirklich oder unwirklich ist - wir haben es nur zu tun mit der subjektiven Wahrheit und Wirklichkeit, nur mit dem Erlebnisfeld des aussagenden Ich selbst“ (Hamburger 1987 [1957], 245), fordert der Modus der Bezeugung im Gegenteil eine Beurteilung in Bezug auf die 
Identität (wem bezeugst du dieses Ereignis?), das Tun (wo warst du im Moment der Tat?) und die Wahrheit, einer Wahrheit also, für die der Autor der Bezeugung verantwortlich gemacht wird. Wir sind weit entfernt von der Konsensproduktion, mit der wir es in der vorherigen Familienstruktur zu tun hatten. Die Rolle der Bedingungen hat sich umgekehrt, der „normale“ Status der Familienkommunikation hat sich verändert: Die neuen Gedächtnisproduktionen erfordern die Debatte, die Diskussion, sogar den Konflikt zwischen Familienmitgliedern und dem sich äußernden Subjekt. Die Familie ist nunmehr ein ausdrücklich manifestierter Spannungsraum. Es ist keinesfalls sicher, dass dies zu einer Auflösung des Familienraums führt. Es kann vielmehr als ein Prozess der Klärung von Beziehungen und als Veränderung der Struktur der Institution angesehen werden.

Definition des Modus der Zeugenschaft

- diskursive Ebene: Vorherrschaft narrativer Strukturen;

- affektive Ebene: extrem starke persönliche Eingebundenheit;

- enunziative Ebene: Konstruktion eines Enunziators ICH, der in Bezug auf Identität, Tun und Wahrheit in Frage steht.

Andere Gedächtnisoperatoren

Während im vorherigen Raum die Bedingungen der Familienstruktur den Status der Operatoren regelten, gelten nun die Bedingungen der Fernsehsprache. Die Filme sind nun nach dem Kanon des Fernsehens gemacht: Interviews der Familienmitglieder, Zooms, die diese und jene Details zeigen (der Zoom ist eine starke Markierung der Enunziation), mündliche Eingriffe, direkt an den Kameramann gerichtet, um diese oder jene Situation zu kommentieren ... Diese Produktionen sind oft montiert: man spielt mit den Übergängen, die die Software bietet, man gibt Anweisungen, man mischt Filme und Fotografien ... Der Familienfilm wandelt sich zur Familienreportage: Eine Produktion, die von ihrem Thema übernommen wird (man spricht dann von Home Video [Moran 2002]). 
Dieses Subjekt richtet sich nicht mehr ausschließlich an die Familie, sondern gleichermaßen an Gefährten, Freunde, Verwandte oder all diese unbekannten Freunde, die man an Orten des Typs Facebook haben kann. Viele dieser Videos werden tatsächlich im Internet, in einem persönlichen Blog oder auf einer Sharing-Plattform veröffentlicht und nehmen so an der Art der Überlagerung zwischen öffentlichem und privatem Raum teil, die für die heutige Gesellschaft charakteristisch ist. Patrice Flichy verwendet einen Begriffsvorschlag des Psychologen Serge Tisseron und spricht vom „extimen Raum“ (Flichy 2010, 45). Tisseron nennt „Begehren der Extimität“ „die Bewegungen, die jeden dazu bringen, einen Teil seines intimen Lebens zu veröffentlichen“ (Tisseron 2001, 52). Diese Videos sind in der Tat ebenso viel, wenn nicht mehr, Selbstausdruck wie Familienproduktionen. Allerdings: Wenn sie einigen der Bedingungen der Familie entfliehen mögen, werden sie doch wieder unter das Joch anderer Bedingungen geraten: die redaktionellen und wirtschaftlichen Modelle, die diese Plattformen beherrschen, Bedingungen, derer sich die Macher dieser Videos übrigens nicht immer bewusst sind.

Die Veränderungen manifestieren sich gleichermaßen auf der Ebene der Operatoren der Rezeption. Heute werden Filme und Familienfotos auf dem Bildschirm des Fernsehens oder jenem des Computers oder sogar auf dem Bildschirm des Handys gesehen. Allgemein diagnostiziert man dieser Veränderung, dem Übergang zu Bildschirmen kleinerer Größen, dass diese die individuelle Lektüre fördere; dies ist jedoch nur zum Teil richtig. Nicht nur werden die Filme und Fotos auf diesen Mini-Bildschirmen sofort nach der Aufnahme mit der Familie gesehen, sondern der Fotoapparat oder das Smartphone wechselt von Hand zu Hand („gib mir den Film“, meint also: gib mir das Smartphone ${ }^{3}$ ). Im Vergleich zum traditionellen

3 In einem Interview während des Pocket Films Festivals 2006 (http://www.festivalpocketfilms.fr/spip.php?article287) erzählte der Regisseur Jean-Louis Boissier von einer Diskussion im Vorjahr: „Weil nicht vorgesehen war, Filme auf der Leinwand zu zeigen, sagte ich zum Publikum: ,Je vous passe le film'. Sowie ich diesen Satz ausgesprochen hatte, dachte ich mir: ,Auch so kann man einen Film zeigen'. Das Smartphone wurde im Publikum vom einen zum anderen weitergereicht.“ [Anm. d. Ü.: Diese Fußnote haben wir von Frank Kesslers Übersetzung des Textes Odin, Roger (2018) „Das Zeitalter der Filmsprache ist angebrochen“, in: Montage AV 27,2, übernommen.] 
Fotoalbum ermöglicht das Mobiltelefon sogar, den Familienkreis auf Abwesende $\mathrm{zu}$ erweitern, indem man die entlegenen Familienmitglieder mit einem Klick verbindet. Mehr als die Individualisierung der Lektüre liegt die wesentliche Veränderung meines Erachtens darin, dass ich auf diesen Bildschirmen noch viele andere Dinge sehe, als nur Familienproduktionen: Fußballspiele, Fernsehnachrichten, Übertragungen der Varietés, Videospiele, meine E-Mails, mein Bankkonto und sogar meine Steuererklärung ... Das hat zur Folge, dass diese Operatoren nicht nur ihre ,heilige ' Dimension verlieren, sondern dass sie alltäglich werden. Ihre Vermehrung verstärkt diesen Prozess weiter. Zuvor haben wir Bilder in relativ kleiner Zahl gemacht und diese wurden zu echten „Erinnerungsorten“ [lieux de mémoire]. Heutzutage zwingt ihre große Anzahl dazu, sie obsessiv auf dem Computer $\mathrm{zu}$ archivieren, sie zu klassifizieren, zu organisieren und $\mathrm{zu}$ hierarchisieren (Software wird ebenfalls angeboten, um bei dieser Aufgabe zu helfen). Das ist eine radikale Änderung der Logik: Man befindet sich in der funktionalen Logik der Datenbank.

Ein Zeichen dafür, dass der Einfluss der traditionellen Familienstruktur nicht vollständig verschwunden ist, dieses Defizit des Heiligen manchmal schlecht gelebt wird, wird durch die erneute Popularität einen der ältesten heute bekannten Gedächtnisoperatoren bezeugt: das in Öl gemalte Porträt. Man schickt ein Foto ein und erhält ein nach diesem Foto gemaltes Porträt zurück. Diese Produktionen können als Rückkehr zur fotografischen Malerei gelesen werden, aber mit dem Unterschied, dass der Schwerpunkt nun auf dem zweiten Begriff liegt, auf der Tatsache, dass es sich um ein Gemälde handelt. OnlineWerbeanzeigen für diese Art von Porträts betonen, dass diese von „Menschenhand“ (wenn nicht Künstler) produziert und signiert wird (Zeichen der Subjektivierung). Das andere Argument ist, dass das gemalte Porträt „ohne Probleme Zeit und Licht widersteht“ und dass „seine Farben in fünfzig Jahren noch so hell sein werden“, während es nichts Verderblicheres gibt als digitale Fotoproduktionen.

Diese Unsicherheit über die Dauerhaftigkeit neuer Gedächtnisoperatoren scheint mir einer der Hauptgründe für die Verbreitung von ,Familienseiten` zu sein, die eine Art Wiederaufleben des „Familienfilms“ der alten Struktur im Internet darstellen. Im Gegensatz zu Home 
Videos haben Familienseiten die Familie als ihren Enunziator. Sie befinden sich an der Kreuzung der redaktionellen Bedingungen der Homepage und der Bedingungen der Familienstruktur. Die Veröffentlichung von Familienbildern dient hier als Garantie für die Erhaltung des Familiengedächtnisses; auf der einen Seite, weil man glaubt, dass eine Website einen zuverlässigeren Speicherplatz als individuelle Lösungen darstellt (eine Überzeugung, die größtenteils unbegründet ist); auf der anderen Seite, und das ist das Wesentliche, weil es die mehr oder weniger unbewusste Idee gibt, dass, wenn diese Bilder von anderen gesehen werden (und im Internet können es tausende Andere sein), werden sie für die Ewigkeit existieren ... In diesem Fall können wir nicht davon sprechen, die Grenze zwischen privatem und öffentlichem Raum zu überschreiten: Wir bleiben im privaten Raum, aber der öffentliche Raum wird zum ultimativen Operator des Familiengedächtnisses.

Einige allgemeine Bemerkungen zum Schluss: Wie der Leser gesehen haben wird, hat die Kommunikation bei diesem Versuch, den Raum des Familiengedächtnisses sowohl in seiner traditionellen als auch in seiner aktuellen Form $\mathrm{zu}$ charakterisieren, eine neue Dimension angenommen: Der Sinn- und Affektproduktion wurde die Produktion von Beziehungen hinzugefügt (die Schaffung einer Familiengeschichte dient der Herstellung eines Konsens, die Arbeit des Gedächtnisses trägt zur kollektiven und/oder individuellen Identitätsbildung bei, die neuen Weisen der Gedächtnisproduktion können Konflikte erzeugen ...). Es ist nicht überraschend, dass diese Dimension in dem Moment erscheint, an dem ich beginne, das Konzept des Kommunikationsraums zu gebrauchen, denn ein Kommunikationsraum ist im Grunde ein Raum von Beziehungen. Diese relationale Dimension kann sogar wichtiger sein als die Dimension der Textproduktion. Darüber hinaus scheint der Kommunikationsprozess auf die Produktion unterschiedlicher Effekte ausgerichtet $\mathrm{zu}$ sein; hier entstehen zum Beispiel identitäre und ideologische Effekte. Die Analyse im Hinblick auf den Kommunikationsraum bereichert das bisher vorgeschlagene semiopragmatische Modell: Kommunizieren bedeutet Sinn, Affekte, Beziehungen und allgemeiner Wirkungen 
hervorzubringen (vgl. Abb. 5 am Ende des Kapitels). Das Modell ist in einem Hin und Her zwischen Theoretisierung und Analyse aufgebaut.

Man kann nun die Fragen auflisten, die man stellen muss, wenn man einen Kontext im Hinblick auf den Kommunikationsraum analysiert:

- Welchen Kommunikationsraum soll man konstruieren? Welche Relevanzkriterien/Achse der Relevanz ist/sind für diese Konstruktion zu wählen?

- Welche Determinanten sind innerhalb des so konstruierten Raumes am Werk?

- Wie werden die Aktanten konstruiert, die in diesen Raum intervenieren? Welche Wechselbeziehungen bestehen zwischen diesen Aktanten?

- Wie werden Kommunikationsoperatoren konstruiert?

- Wie entsteht die Sinn-, Affekt-, Beziehungs- und Wirkungsproduktion?

- Welche Wichtigkeit haben diese Produktionen?

SchließSlich zeigt dieses Kapitel die Notwendigkeit, die diachrone Entwicklung von Kontexten zu berücksichtigen: Die Veränderung von Bedingungen innerhalb desselben Kontexts (hier die Familie) kann tatsächlich dazu führen, dass ein neuer Kommunikationsraum konstituiert werden muss, obwohl wir für die Analyse auf der gleichen Achse der Relevanz (die Erinnerung) blieben. Denn die kommunikative Erfahrung, mit der wir es zu tun haben, ist nicht mehr die gleiche: Die Aktanten und die Operatoren sind anders konstruiert, die Produktion von Bedeutung geschieht nicht mehr in der gleichen Weise, die Textproduktion hat einen anderer Status, die relationalen Effekte sind nicht mehr dieselben. 


\section{[Schema 5]}

\begin{tabular}{|c|c|}
\hline Raum der Sendung (S) & Raum der Rezeption (R) \\
\hline S- - $--\rightarrow \mathrm{T} /$ Bez./Effekte & VT'/Bez./Effekte $4-\mathrm{R}$ \\
\hline Allgemeine Bedingungen \\
\hline \multicolumn{2}{|c|}{ Sprachliche Bedingungen } \\
Kommunikative Kompetenz als Reservoir von Modi \\
\hline \multicolumn{2}{|c|}{ Kommunikationsräume } \\
Auswahl der Achse der Relevanz \\
Aufzeigen der Bedingungen \\
Konstruktion der Aktanten, der Operatoren \\
Auswahl des oder der zu benutzenden Modi \\
\hline
\end{tabular}

T: textliche Produktion

Bez.: Beziehungen 


\section{Kapitel 5}

\section{Kommunikationsraum}

\section{und Migration in neue Kontexte Das Beispiel des Familienfilms}

Im letzten Kapitel habe ich gezeigt, wie eine Analyse, die mit dem Konzept des Kommunikationsraums operiert, es ermöglicht, das Funktionieren von Produktionen, die zur gleichen Relevanzachse gehören, innerhalb eines gegebenen Kontexts, zu beschreiben und dabei dem historischen Wandel dieses Kontexts Rechnung zu tragen. Dieses Kapitel soll darlegen, inwieweit derselbe Begriff erklären kann, was mit einer Produktion passiert, wenn sie aus ihrem ursprünglichen Kontext heraus migriert. Um diese Frage zu beantworten (und um an das vorige Kapitel anzuschließen), wende ich mich wiederum dem Beispiel der audiovisuellen Familienproduktionen und insbesondere dem Familienfilm zu. Der Familienfilm erfährt in der Tat ein bemerkenswertes Phänomen der Migration durch viele Kontexte hindurch. Es geht hier nicht darum, diese Kontexte selbst zu untersuchen, sondern nur darum, was sie mit den Familienfilmproduktionen machen, die in sie einwandern. Es geht auch nicht darum, alle Kontexte $\mathrm{zu}$ analysieren, in die diese Produktionen migrieren; ich werde mich auf einige Beispiele beschränken, die aufgrund ihrer Vielfalt und ihres methodologischen Belangs ausgewählt wurden.

\section{Der Familienfilm: Vom Archiv zum Erinnerungsort}

Die bemerkenswerteste Manifestation der Migration von audiovisuellen Familienproduktionen außerhalb ihrer Entstehungsinstitution ist sicherlich die Schaffung spezialisierter Archive oder zumindest 
von Archiven, die eine besonders diesen Produktionen gewidmete Sammlung erschließen: Cinémathèque de Bretagne, Vidéothèque de la Ville de Paris, Médiathèque de Saint-Étienne, Cinémathèque d'Andalousie, Cinémathèque Basque, Musée d'Ethnographie de Conches (Schweiz), North West Film Archive (Manchester), Scottish Film Council (Glasgow), Small Film Museum (Niederlande), The New Zealand Film Archive, Österreichisches Filmmuseum (Wien), Bophana Audiovisual Resource Center (Kambodscha), National Board of Antiquities for Prints and Photographs (Finnland), The Living Picture Archive (Viborg, Stifts Museum, Dänemark), The Human Studies Film Archives (Smithsonian Institution, Washington D.C.) etc.

Ihr Wert als Dokument rechtfertigt die Migration von Familienproduktionen in diese Archive. Im dokumentarisierenden Modus gelesen, liefern sie in der Tat wertvolle Informationen über ganze Teile der Gesellschaft, die nicht von offiziellen Stellen oder professionellen Berichten dokumentiert werden; unübertroffen sind sie insbesondere in der Dokumentation dessen, was passiert, wenn nichts passiert, „das Banale, das Alltägliche, das Allgemeine, das Gewöhnliche, das InnerGewöhnliche [l'infra-ordinaire] ${ }^{1}$, das Hintergrundgeräusch, das Übliche“. Georges Perec, von dem ich mir diese Aufzählung leihe, stellt fest: „Die Zeitungen sprechen von allem, außer dem Alltäglichen“, und fragt sich: „Was wirklich vor sich geht, was wir erleben, der Rest, alles andere, wo ist das?“ Dann beginnt er von einer „endotischen Anthropologie“ (vs. einer exotischen) zu träumen (Perec 1975, 251-255). Familienfilmer sind auf ihre Weise unfreiwillige endotische Anthropologen. Ohne daran zu denken, zu dokumentieren, filmen sie jene Momente des Lebens, die professionelle Filmschaffende nicht filmen (weil sie nicht Teil des Kommunikationsraums sind, in dem sie arbeiten).

Darüber hinaus sind die Institutionen, die diese Produktionen sammeln, meist regionale Archive oder Filmbibliotheken, die als solche besonderen Bestimmungen unterliegen: Insbesondere sind sie an den

1 [Anm. d.Ü.] Der Begriff „infra-ordinaire“ von Georges Perec impliziert die Forderung nach einem wahrhaften Journalismus, der gerade nicht das Außergewöhnliche [frz.: extraordinaire], sondern das Inneralltägliche oder eben 'Innergewöhnliche' zum Thema macht und damit der Realität näherkommt, als die auf das Spektakel gerichtete Berichterstattung. 
Identitätsfragen der Region beteiligt. In mehr oder weniger ritualisierten Filmvorführungen kommen Menschen zusammen, um eine Geschichte zu teilen und zu zeigen, dass sie zur gleichen Gemeinschaft gehören; die Sinn- und Affektproduktion findet dann in Beziehung zum Gedächtnis der Gruppe statt. Wir sind nicht mehr im dokumentarisierenden Modus, sondern im privaten Modus. Die relationale Dimension der Kommunikation hat dann Vorrang vor der Sinnproduktion. Archive werden zu „Erinnerungsorten“ (Nora 1990 [1984]). Der am häufigsten von den Hinterlegenden für die Hinterlegung von Filmen angegebene Grund besteht übrigens im Wunsch, am Gedächtnis der Region teilzuhaben.

Die Wahl zwischen dokumentarisierendem und privatem Modus hängt wesentlich vom Status des Aktanten ab, der an diesen Filmen interessiert ist. Während die Bewohner der Region als Mitglieder einer Gemeinschaft ins Archiv kommen (privater Modus), mobilisieren die Forscher (Historiker, Soziologen, Ethnologen, Anthropologen, andere einfach Neugierige) vorrangig den dokumentarisierenden Modus: Die Frage nach der Wahrheit ist für sie wesentlich. Offensichtlich kann aber dieselbe Person auch beide Aktantenrollen besetzen.

Je nachdem, ob die Sinnproduktion nach dem einen oder dem anderen Modus erfolgt, werden nicht dieselben Operatoren mobilisiert. Wenn der private Modus dominiert, finden wir die gleiche Art von Operator wie in der Familie, aber auf der Ebene einer größeren Gemeinschaft (einer Stadt, einer Region): Filme als Stimulatoren des Gedächtnisses und der Beziehungen. Wichtig ist weniger das, was sie zeigen oder sagen, als die Erinnerungsarbeit, die sie hervorrufen, und die Verbindung, die sie zwischen den Rezipienten schaffen (oder verstärken). Wenn umgekehrt der dokumentarisierende Modus dominiert, werden dieselben Filme eher wie Träger von Information aufgefasst und die Operatoren haben einen ganz anderen Status: Sie sind die Werkzeuge, Analyse-Operatoren, die es ermöglichen, die Vergangenheit in einer (mehr oder weniger) systematischen, durchdachten, distanzierten Weise zu rekonstruieren (was das Gedächtnis nicht tut). 
Hier sind einige Beispiele für Analyse-Operatoren:

Die Oberflächenanalyse: Es geht darum, sich auf Dinge zu konzentrieren, die der Film zeigt, ohne dass diese selbst Gegenstand der Dreharbeiten waren: die Landschaft, der Lebensraum, die Schaufenster und Aushängeschilder in Läden, die vorbeifahrenden Autos, die Kleidung der Figuren, Nebensächlichkeiten (der Polizist, der den Verkehr regelt, der Straßenkehrer, der Zeitungsverkäufer) usw.

Das Erstellen von Serien: Die Konfrontation von Darstellungen desselben Themas (der Status der Frau, der Urlaub, die Ehe) in Filmen aus verschiedenen Epochen und Kulturen ermöglicht es, Unterschiede aufzudecken und die Lektüre produktiv zu machen.

Die enunziative Analyse: Es geht um den Standpunkt, aus dem die Filme die Welt zeigen: Wie filmen Kolonialisten Afrika und Afrikaner? Gibt es eine männliche Art zu filmen? Macht ein Protestant die gleichen Familienfilme wie ein Katholik?

Die Kontextualisierung: Das Dargestellte kann undurchsichtig bleiben oder zumindest nicht seine ganze Bedeutung entfalten, wenn es nicht in einen Kontext gestellt wird. Es ist dann notwendig, den Autor der Bilder um Informationen zu bitten, den Film in seinen historischen und sozialen Rahmen zu stellen, kurz gesagt, den Film zu verlassen, um besser zurückzukehren und ihn besser zu verstehen.

Die Veränderung des „Rahmens“ im Sinne von Goffman (1977 [1974]): Ein Film kann wichtig werden, weil sich der historische Lektürerahmen verändert hat. So erzählt André Huet, der Gründer der Association Inédits - einer internationalen Vereinigung, die seit 1991 all jene Archivare, Regisseure und Forscher zusammenbringt, die sich für Familienfilme als Dokumente interessieren -, wie Filme von Reisen nach Jugoslawien, die er gelagert hatte, die er aber für uninteressant hielt, nach dem Krieg zu bemerkenswerten Dokumenten wurden.

Es sollte hinzugefügt werden, dass die Analyse-Operatoren je nach dem disziplinären Rahmen, in dem die Sinnproduktion stattfindet, unterschiedlich sind: Historiker, Soziologen, Ethnologen, Anthropologen verwenden nicht die gleichen theoretischen und methodischen Werkzeuge. 
Kurzum, audiovisuelle Familienproduktionen erscheinen in diesem Zusammenhang als Teil zweier Kommunikationsräume:

- einerseits des Kommunikationsraums des Dokuments: Die Sinnproduktion erfolgt dort im dokumentarisierenden Modus, die Aktanten der Kommunikation verhalten sich als „Forscher“, die die Analyse-Operatoren mobilisieren, um aus Filmen Informationen zu erhalten.

- andererseits des Raums des kollektiven Gedächtnisses: Der Operator Film fungiert als Stimulator (privater Modus) und die Aktanten treten als Mitglieder einer Gemeinschaft auf.

Es kann durchaus passieren, dass diese Räume autonom vorkommen, aber meistens gibt es eine Überschneidung: Der ehemalige Bergmann, der in die Mediathek von Saint-Etienne kommt, um an einer Vorführung von Amateurfilmen aus den 1950er Jahren teilzunehmen, wird sich zweifellos von der Erinnerungs- und Gemeinschaftsdimension mitreißen lassen, aber er wird sicher gleichzeitig etwas über seine Stadt erfahren. Ebenso wird der Historiker, der an einem Korpus von Familienfilmen arbeitet, um das Leben der Bergleute im Département Loire zu erforschen, zweifellos das Bedürfnis verspüren, um seine Analyse zu erweitern, sein eigenes Gedächtnis zu mobilisieren und an die Erinnerung der Menschen zu appellieren, die es gelebt haben.

Anmerkung: Man kann sich fragen, ob es nicht sinnvoller gewesen wäre, nur einen einzigen Kommunikationsraum, den Raum des Archivs, zu konstruieren und in ihm zwei Pole zu etablieren, je nachdem, ob die Kommunikation eher zum Gedächtnis oder eher zum Dokument tendiert. Diese Lösung hätte sicherlich den Vorteil, die Einheit des Archivkontextes zu markieren, sie hat aber auch zwei Nachteile. Zum einen ordnet sie Gedächtnis und Dokument derselben Achse $\mathrm{zu}$, als handele es sich um Erfahrungen gleicher Art, was zumindest zu diskutieren wäre. ${ }^{2}$ Auf der anderen Seite verbindet es diese beiden Begriffe, indem es sie an den beiden Polen derselben

2 Vgl. die Auseinandersetzung um das Verhältnis von Geschichte und Gedächtnis: Nora 1990; Ricœur 2004 [frz. 2000]. 
Achse anordnet, und schließt damit aus, dass einer von ihnen ohne jede Beziehung zum anderen funktionieren könnte (sogar wenn es sich um eine Oppositionsbeziehung handelt). Zwar mag man meinen, dass der Gedächtnisansatz sehr häufig mit einer gewissen Dosis Dokumentationsansatz kombiniert wird, der Dokumentationsansatz scheint jedoch auch unabhängig zu funktionieren.

\section{Der Familienfilm im Fernsehen}

Nach den Archiven ist das vielleicht auffälligste Phänomen in Bezug auf die Migration von audiovisuellen Familienproduktionen außerhalb des familiären Kontexts ihre äußerst beharrliche Präsenz im Fernsehen, in Fernsehnachrichten, in Magazinen und Talkshows (das Fernsehen kann keinen Schriftsteller, Maler, Sportler, Politiker oder Gelehrten mehr empfangen, ohne Ausschnitte seiner Familienfilme zu zeigen), ganz zu schweigen von VIDÉo GAG ${ }^{3}$ (F [TF1] 1990-2008).

Wenn wir VIDÉo GAG, das aus einem anderen Raum kommt, vorübergehend ausschließen, könnte diese Migration als eine Passage im Raum des Dokuments und damit als Einladung zur Lektüre dieser Produktionen im dokumentarisierenden Modus beschrieben werden. Diese Sichtweise ist zwar nicht ganz falsch, verfehlt jedoch das Wesentliche: Neben der Tatsache, dass der Informationsgehalt der gesendeten Filmfragmente oft extrem gering ist, kann diese Migration nur verstanden werden, wenn sie in der Perspektive des Übergangs vom Paläo- zum Neofernsehen, d. h. im Kontext eines Strukturwandels und damit von Bedingungen im Fernsehen selbst betrachtet wird. In den 1980er Jahren führten wirtschaftliche und politische Veränderungen dazu, dass das Fernsehen eine bestimmte Art von Beziehung zum Zuschauer favorisierte: Die für das Paläo-Fernsehen charakteristische pädagogische (hierarchische) Beziehung wurde durch eine

3 [Anm. d.Ü.:] VIDÉO GAG war eine französische Fernsehsendung, in der „komische“ Ausschnitte aus Familienfilmen präsentiert wurden. Vergleichbare Formate im deutschen Fernsehen waren und sind Pleiten, Pech und PANnen (Das Erste 1986-2003), Bitte LächelN (Tele 5, bzw. DSF und RTL II 1990-1998), BITTE LACHEN! (RTL II seit 2005). In beinah epidemischer Ausbreitung finden sich solche Formen weiterhin in den OnlineVideoportalen. 
Beziehung der Nähe ersetzt (Casetti/Odin 2001 [1990]). Der Rückgriff auf die Familienproduktionen ist eine Fortsetzung dieser Bewegung: Sie fungieren als Operator der Nähe. Wenn ich zum Beispiel die Familienfilme der eingeladenen Persönlichkeiten sehen darf, bringt das Fernsehen sie mir näher, denn diese Filme ähneln denen meiner Familie. Die Sinnproduktion erfolgt dann im intimen Modus: Ich suche in meinem eigenen Leben, was ich mit diesen Gästen teile. Dann kann eine empathische Beziehung hergestellt werden.

Aber noch wichtiger als ihr Inhalt ist der Ursprung dieser Bilder. Die Macher dieser Sendungen versäumen nicht, es zu unterstreichen (oft mit einem Untertitel: „Amateurbilder“), sicherlich um die schlechte Qualität dieser Bilder zu rechtfertigen, aber vor allem, weil die Erwähnung dieser Herkunft als ein enunziativer Operator fungiert, der mich einlädt, diese Bilder als von Menschen „wie mir“ (anstatt von Profis) aufgenommene Bilder zu sehen. Von da an fordern mich diese Bilder anders heraus: Sie besitzen eine spezifische emotionale Kraft, eine Kraft, die mich drängt, sie so zu akzeptieren, wie sie sind, ohne ihren Enunziator in Bezug auf die Wahrheit in Frage zu stellen (ihr Ursprung ist das Versprechen ihrer Unschuld). Als Modus der Authentizität bezeichne ich den Modus, der mich zwar einlädt, einen realen Enunziator zu konstruieren, mir aber verbietet, ihn in Bezug auf die Wahrheit in Frage zu stellen.

\section{Definition: Modus der Authentizität}

- enunziative Ebene: Konstruktion eines realen Enunziators, der auf der Identitätsebene als „wie ich“ konstruiert wird;

- diskursive Ebene: Jede Textproduktion ist möglich;

- affektive Ebene: Die Tatsache, dass dieser Enunziator „wie ich“ ist, führt zu einer affektiven Beziehung, die jede Infragestellung in Bezug auf die Wahrheit verbietet.

Der Modus der Authentizität steht dem dokumentarisierenden Modus entgegen.

Für die betrachteten Sendungen ist es wichtig, den Zuschauer zu ermutigen, den Modus der Authentizität im Inneren des Raums des Dokuments und damit in Konkurrenz zum dokumentarisierenden 
Modus anzuwenden. Der Modus der Authentizität untergräbt also den Raum des Dokuments von innen: Er hat nichts mit der Frage nach der Wahrheit zu tun. Es scheint mir, dass eine der Hauptfunktionen des Gebrauchs von audiovisuellen Familienproduktionen im Fernsehen, aber auch in vielen anderen Zusammenhängen, darin besteht, die Möglichkeiten des kritischen Denkens einzuschränken.

Als Beweis für die Stärke des Modus der Authentizität zögert die Werbung nicht, gefälschte Familienfilme (erkennbar an ihrem Thema, aber auch an unscharfen, wackeligen, schlecht kadrierten Bildern, dem Klang des Projektors usw.) herzustellen, um ihn zu mobilisieren. Das Verfahren besteht darin, den Kreuzungspunkt zwischen Familienraum und Werberaum zu bespielen: Verwendung von „gebrauchsfertigen“ Szenarien des Familienlebens, die sich jedoch auf das zu bewerbende Produkt konzentrieren; Verwendung der fiktionalen Kommunikationstrope: Man zeigt als Zielgruppe eine fiktive Familie, um den wahren Adressaten, den kaufenden Zuschauer, anzusprechen. Wir werden so dazu gebracht, den Standpunkt dieser Filme als vollkommen objektiv zu betrachten ... „da es der unsere ist“ (Journot/ Duchet 1995, 182).

Es ist gleichermaßen der Bezug zu diesem Modus, der eine Sendung wie VIDÉo GAG zu etwas anderem macht als zu einem Programm, das zum Raum der Unterhaltung gehört, dem es dennoch zweifelsohne angehört. VIDÉO GAG ist ohne Frage die populärste Sendung in Bezug auf die Migration von Familienfilmen ins Fernsehen: Seit seiner Premiere im September 1990 ist es ein ungebrochener Erfolg in Frankreich. Es scheint, dass es die japanische Sendung KaTO-CHAN KEN-CHAN GOKIGEN TV (JPN [TBS] 1986-1992) war, die das Konzept Mitte der 1980er Jahre einführte. Nun gibt es sie in Variationen überall auf der Welt: AMERICA's FunNIEST HOME VIDEOS (USA [ABC] seit 1989/1990), You've BeEN FrAmed! (GB [ITV] seit 1990), DRÔLE DE VIDÉo (CAN [TVA] 1990-1999) etc.

In gewisser Weise sagt der Titel des Programms alles: Es geht darum, Familienfilme drastisch auf Gags zu reduzieren. Der Operator ist sowohl einfach als auch bemerkenswert wirkungsvoll: Fragmente von Familienfilmen werden für die Gags, die sie zeigen, ausgewählt; diese Gags werden dann in Serie gesetzt: Stürze, Schläge, Hochzeiten, 
Tiere, Kinder ...; schließlich werden Soundeffekte sowie ein Kommentar (oft in Dialogform) hinzugefügt, um die komische Dimension der Situationen zu verstärken. Die so transformierten Fragmente von Familienfilmen sollen im spektakularisierenden Modus gelesen werden: In ihrer Erscheinung haben sie keine andere Funktion, als uns zum Lachen zu bringen. Doch im Raum der Rezeption verhält sich dies nicht ganz so: Sicherlich wird der spektakularisierende Modus durchaus angewandt, man lacht viel, aber was sich abspielt, geht weit über dieses Lachen hinaus. Das Spektakel, das ich auf dem Bildschirm sehe, macht meine Mitmenschen (ich bin einer von ihnen) zum Enunziator und das in dreifacher Hinsicht: als Darsteller der Gags, als Verantwortliche für die gefilmten Bilder und als Verantwortliche für den Entschluss, diese Bilder ans Fernsehen zu schicken. Diese besondere enunziative Beziehung widerspricht der Wirkung der Distanz des spektakularisierenden Modus und drängt mich, den Modus der Authentizität zu mobilisieren und die unbestreitbare Wahrheit der mir gegebenen Bilder zu erkennen: Diese Serie von Gags, die ein lächerliches, groteskes und bedauernswertes Bild von den anderen und mir selbst zeichnen - sagen die Wahrheit. VIDÉO GAG ist keine unschuldige Sendung. Sie macht mich nicht nur zu einem Teilnehmer an der universellen Dummheit, sondern lädt mich ein, sie ohne Diskussion zu akzeptieren, schlimmer noch, sie in einer radikalen Übung der Selbstverachtung zu genießen.

Diese Analysen gelangen zum gleichen Schluss: Durch die Förderung der Selbstverachtung, durch das Blockieren von Wahrheitsfragen (dokumentarischer Raum und Raum der Werbung) spielt die Migration des Familienfilms ins Fernsehen eine ideologische Rolle: die eines Instruments zur Reduzierung des kritischen Bewusstseins. Indem sie dies tun, greifen die Familienfilme auf ihre primären Funktionen im Raum der Familie zurück: Probleme vermeiden, Konsens stiften, die Institution fortbestehen lassen. Familienproduktionen appellieren so selbstverständlich an den Modus der Authentizität, dass man von einem Familienfilmeffekt sprechen kann, um diese Film-Zuschauer-Beziehung zu bezeichnen, die durch die Blockade der Wahrheitsfrage gekennzeichnet ist. 


\section{Vom Familienfilm zur Mikro-Geschichte}

Wie wir während der Beschäftigung mit den Archiven gesehen haben, ist der Familienfilm eine sehr gute dokumentarische Quelle. Kein Wunder also, dass Historiker oder Bürger, die der offiziellen Geschichtsschreibung ihres Landes entfliehen wollen, beschließen, sie für die Vermittlung von Geschichte zu nutzen. Aber wie kann man dem Familienfilmeffekt entgehen, wenn man diese Art von Dokument zur historischen Reflexion verwendet?

Péter Forgács' Serie PrIVÁt MAGYARORSZÁG (Ungarn privat, HUN 1988-2008, 14 Filme) erscheint mir für die Beantwortung dieser Frage beispielhaft. Diese Serie umfasst mehr als ein Dutzend Langfilme, die ausschließlich durch die Bearbeitung von Familienproduktionen entstanden sind (wir befinden uns also in der Tradition des Found Footage Film ${ }^{4}$ ). Der Entstehungskontext dieser Filme erlaubt eine Hypothese aufzustellen, die den Einsatz dieser Verfahrensweise durch den Regisseur erklärt: In seiner Geschichte konfrontiert mit der Frage nach der nationalen Existenz innerhalb eines Imperiums, das seit vielen Jahren mehreren Besetzungen ausgesetzt ist, ist Ungarn ein Land mit einem zerrissenen Gedächtnis. Wenn es wahr ist, wie Pierre Nora sagt, dass es „lieux de mémoire [gibt], weil es keine milieux de mémoire mehr gibt" (Nora 1990 [1984], 11), kann man verstehen, dass sich ein ungarischer Regisseur, der die Geschichte seines Landes hinterfragen will, Familienfilmen zuwendet, diesen fabelhaften Erinnerungsorten. Außerdem ist es nicht absurd zu glauben, dass eine Änderung des Maßstabs es ermöglicht, die Dinge anders zu betrachten als die offizielle Geschichtsschreibung. Wir erkennen hier die Problematik der Mikro-Geschichte (Revel 1996).

Der erste Film der Serie, A Bartos család (Die Familie BaRTos, HUN 1988), den ich hier als Beispiel nehme (er veranschaulicht das Prinzip der ganzen Serie), fordert ausdrücklich seine Einschreibung in diesen

4 [Anm. d. Ü.:] Odin entscheidet sich mit der Kennzeichnung „Found Footage“, die Filme dem Kontext des Experimentalfilms zuzuordnen und nicht, wie es der Begriff „Kompilationsfilm“ naheläge, dem Dokumentarfilm; vgl. in diesem Zusammenhang Steinle, Matthias (2007). Das Archivbild und seine ,Geburt' als Wahrnehmungsphänomen in den 1950er Jahren. In Müller, Corinna / Scheidgen, Irina (Hg.): Mediale Ordnungen. Erzählen, Archivieren, Beschreiben. Marburg: Schüren, S. 259-282. 
Strang der historischen Forschung ein: „Die Bartos-Saga ist ein ungarischer Familienroman, die Spiegelung einer privaten Geschichte“ (Kommentarstimme im Film).

Forgács' filmische Arbeit - die Verwendung von Untertiteln, die Zerlegung von Bewegungen, Standbild, Zeitlupe, die Wiederholung von Sequenzen, repetitive Musik - fungiert als enunziativer AnalyseOperator. Seine Aufgabe besteht darin, den Standpunkt des Regisseurs explizit werden zu lassen: „Die Welt gesehen von Zoltán Bartos“, wie die einleitende Sequenz des Films sagt. Diese programmatische Aussage markiert den Willen, den Vermittlungsprozess zum zentralen Thema des Films zu machen. Die übliche Funktionsweise kinematografischer Referenz wird hierdurch modifiziert: Statt einer präsenten Realität werden uns Repräsentationen gezeigt. So ist es unmöglich, mich in den Lektüremodus der Authentizität übergehen zu lassen.

Einerseits werde ich zu einer Lektüre im diskursiven Modus gebracht:

\section{Definition des diskursiven Modus}

- diskursive Ebene: Konstruktion eines argumentativen Textes;

- affektive Ebene: Die Affekte werden verwendet, um zu überzeugen (wir befinden uns im Bereich der Rhetorik);

- enunziative Ebene: Konstruktion eines realen Enunziators, der in Bezug auf Identität, Tun und Wahrheit befragbar ist.

Andererseits greift der diskursive Modus hier auf der Metaebene ein: Ich bin eingeladen, mich zu fragen, was die Besonderheit der Welt von Zoltán Bartos ausmacht.

Der Film liefert sehr schnell zwei Antworten: Für Zoltán Bartos besteht die Welt in erster Linie aus seiner Familie und dem Familienunternehmen. Der Vater, „das Familienoberhaupt“, ist auch „Vorsitzender einer Holzverarbeitungsgesellschaft“. Wir begleiten ihn auf einer Führung durch seine Werkstätten am Donauufer mit seinem Verwaltungsrat. Es ist selten in einem Familienfilm, Sequenzen zu finden, die der Arbeit gewidmet sind. Die Tatsache, dass Zoltán Bartos beschlossen hat, einen solchen Besuch zu filmen, ist gewiss bezeichnend für die bürgerliche Mentalität. Aber am interessantesten ist die Art und Weise, wie uns die Beziehung zur Arbeitswelt gezeigt wird. Während des 
Besuchs führt uns der Film in die Werkstätten, in denen die Arbeiter mit dem Sägen von Brettern beschäftigt sind. Während sich der Film bis dahin damit begnügte, uns Musik hören zu lassen, gibt er nun den Lärm der Werkstätten wieder, insbesondere den schrillen Klang der Sägen. Ein wenig später, zwischen zwei Einstellungen, in denen Bartos vor seinem Laden posiert, zeigt der Film Holzarbeiter, die riesige Bretter tragen, um sie in einen Wagen zu legen und wiederum bekommen wir den Klang der Bretter, wie sie in den Wagen fallen, zu hören. Da das damalige Amateurkino stumm war, weiß der Betrachter, dass diese Geräusche das Ergebnis von Postproduktionsarbeiten sind und daher keinen anderen Enunziator als Forgács haben können; deshalb kann er für sie nur eine bewusste Bedeutung suchen, die über ihre einfache diegetische Verankerung hinausgeht, zumal die Montage zum Aufbau eines Oppositionssystems einlädt: Wenn die Familie Bartos mit Musik in Verbindung gebracht wird, wird der Lärm mit der Arbeit der Arbeiter in Verbindung gebracht, als ob wir plötzlich in die Realität zurückfielen, in das Konkrete (jeder Lärm ruft seine Quelle hervor). Ohne es explizit zu machen, treibt der Film den Zuschauer zu der Erkenntnis, dass es die sehr reale Arbeit anderer ist, die es der Bourgeoisie erlaubt, „gut zu leben“ („er [Bartos] hatte eine Fabrik und ein Geschäft, die es ihm erlaubten, gut zu leben“).

Andererseits betonen einige Sätze des Kommentars die historischen Ereignisse in der Zeit der vorgeführten Bilder des bürgerlichen Tanzens, Trinkens und Essens (die Invasion Österreichs durch Hitler, die Gesetze gegen Juden usw.), um uns die Gleichgültigkeit der bürgerlichen Gesellschaft gegenüber der Geschichte begreifbar zu machen. Der Film zeigt, dass die Bourgeoisie nichts kommen sah, weder die Nazis noch den Kommunismus. Die Schlusspassage des Films dreht sich direkt um diese Vergessenheit der Geschichte: Während wir an der kommunistischen Parade zum 1. Mai teilnehmen, lässt uns Péter Forgács ein Lied von Kazal hören: „Wann hat Napoleon eine große Schlacht gewonnen oder verloren? In welchem Jahr war er Kaiser? Wann wurde er belagert? Es bringt nichts, mich zu fragen, ich kann nichts darauf antworten, denn ich konnte mir nie ein historisches Datum merken.“ 
Doch damit nicht genug: Während die Lektüre im Modus der Authentizität blockiert wurde, hat Péter Forgács sehr wohl das Potential des emotionalen Inhalts dieser Bilder zu nutzen gewusst. Er bedient sich dieser (im doppelten Sinne des Wortes) vergangenen Bilder, um ihre figurative Dimension zu befreien und dabei gleichzeitig eine Reihe von Prozessen in Gang zu setzen, die uns zwingen, uns zu distanzieren. Es geht darum, dieses affektive Potenzial in den Dienst der Reflexion $\mathrm{zu}$ stellen. Die Musik des im Film angegebenen Komponisten Tibor Szemzö fungiert als eine Art Kommentar zu den Bildern; sie ist die Stimme der Geschichte, eine Stimme, die uns dazu bringt, diese Bilder in Frage zu stellen, indem sie uns in die Zukunft versetzen (in Bezug auf zukünftige Katastrophen erzeugen sie Sinn). Die Geräusche, die uns auch aufgrund ihrer Seltenheit auffallen, werden gleichfalls in dieser vorwarnenden Weise eingesetzt (die Zugsequenzen) oder betonen in den Bildern die Probleme, die wir ohne sie nicht gesehen hätten: das Problem des Verhältnisses der Bourgeoisie zur Realität, das Problem der Klassenverhältnisse etc. Was den spärlichen Kommentar angeht, so ist dieser unter dem Deckmantel sehr einfacher Strukturen (oft kurze Nominalsätze) bei weitem nicht neutral; diese kleinen Sätze sagen gleichzeitig zu viel und zu wenig und veranlassen uns, den Diskurs selbst $\mathrm{zu}$ bilden. Der Film konfrontiert uns mit einem fragmentarischen, unvollständigen, manchmal scheinbar ungeordneten Text, den wir vervollständigen und organisieren müssen. Die ganze filmische Arbeit von Forgács ist also darauf ausgerichtet, uns zu zwingen, uns Fragen über die Bilder zu stellen, die er uns zu sehen gibt. A BARTOS CSALÁD gehört zur Kategorie der stimulierenden Filme (um eine Formulierung von Alain Resnais über seinen Film MURIEL OU LE TEMPS D'UN RETOUR [F 1963] zu verwenden). Er versucht, uns in die historische Reflexion einzubeziehen, eine Reflexion, die weit über den Fall Bartos hinausgeht, da sie die Position der Bourgeoisie in der Geschichte betrifft.

In A BARTOS CSALÁD blockiert die Verwendung von Familienfilmen keineswegs die Frage nach der Wahrheit, das Zentrum der Anordnung, aber dieses Ergebnis hat eine größere filmische Arbeit erfordert. Diese Arbeit besteht darin, den Familienfilm als ideologischen Operator zu analysieren, der das Verhalten einer sozialen Klasse aufzeigt. Der 
Übergang $\mathrm{zu}$ einem Kommunikationsraum auf der Metaebene ermöglicht eine solche Analyse: Die filmische Arbeit betreibt die Schaffung eines kritischen „Diskurs“-Raumes gegenüber dem Raum des Familienfilms. Der Film von Forgács ist eine Art semio-historische Analyse von Familienfilmen durch den Film.

\section{Der Familienfilm im Raum der Kunst}

Der Film A SONG OF AIR (AUS 1988) der Australierin Merilee Bennett präsentiert sich wie ein Brief an ihren verstorbenen Vater. Eine von Merilee selbst handbeschriebene Schrifttafel teilt uns am Anfang des Films mit, dass das Bildmaterial dieses Brief-Films aus den Familienfilmen zusammengesetzt ist, die ihr Vater aufgenommen hat - der hochwürdige Arnold Lucas Bennett, der seine Familie zwischen 1956 und 1983 mit anhaltender Regelmäßigkeit filmte. Aus den uns angebotenen Bildern geht hervor, dass der Vater Merilees offenbar ein „guter Filmemacher" war: Nicht nur sind seine Bilder scharf und wohl kadriert, seine Filme sind auch sorgfältig konstruiert und sogar inszeniert: „In den Ferien hat er uns versammelt, um in seinen Filmen zu spielen. Wir simulierten Urlaubsabfahrten mit Abschiedsszenen, in denen wir sahen, wie das Auto angelassen wurde und dann wegfuhr“, erläutert der Kommentar. Der Vater schuf sogar Drehbücher, die „alle das gleiche Thema hatten: eine von äußerer Gefahr bedrohte Familie“. „Wir spielten unser Leben seinen Absichten entsprechend. Wichtig war es, zusammen zu sein, die Welt auf die gleiche Weise zu sehen.“ Automatisch aufgenommene Bilder zeigen uns den Vater, der von seiner Frau und seinen Kindern umgeben ist, sie unter seine Fittiche nimmt, sie mit seinen großen Armen umfängt, sie zu einem stilisierten Gemälde eines Familienfotos organisiert und sie ständig einlädt, in die Kamera zu schauen: In die Kamera zu blicken, heißt gemeinsam in dieselbe Richtung zu gucken und so die Einheit der Familiengruppe zu bezeugen. Die Form entspricht hier genau dem Inhalt. Geordnete, geregelte, zivilisierte, fast polizeiliche Bilder: Bilder der familiären und moralischen Ordnung, die der Vater (ein überzeugter Baptist) unnachgiebig in seiner Familie durchsetzt. 
Das Ergebnis dieser Erziehung, bei der das Heimkino eine wichtige Rolle spielt - „Sonntags, nach dem Tee, sahen wir die Filme, sahen wir uns selbst aufwachsen ..." - wird durch den Text des von der OffStimme Merilees vorgelesenen Briefes wiedergegeben: Es handelt sich um die Erzählung ihrer Revolte sowohl gegen die familiäre Ordnung als auch gegen die Filme, die gleichermaßen Spiegelbild und Agent dieser Ordnung sind. In diesem Brief erklärt Merilee ihrem Vater, wie und warum sie sich in ein Leben stürzte, das genau das Gegenteil von dem war, was er für sie geplant hatte, wie sie eine Oben-OhneKellnerin in einem Bordell wurde, wie sie sich prostituierte und betäubte.

Angesichts eines Familienfilms, der „gut gemacht“ und damit gegenüber seinem familiären Publikum gewalttätig ist, kann es nur andere Gewalt ermöglichen, aus ihm herauszukommen. Diese Gewalt ist hier die der Kunst. Die Bilder der väterlichen Filme werden durch Merilee nicht nur neu geschnitten und montiert, damit diese in ihren Diskurs einfließen, sondern sie führt auch eine kinematografische Arbeit aus, die ein Gegenspiel zu der von ihrem Vater durchgeführten Arbeit darstellt: Es geht darum, die zu gut kadrierten, zu scharfen und zu sauberen Bilder des Vaters zu zerstören, indem man sie durch die Zersetzung und Neuzusammensetzung von Bewegungen oder durch den Angriff auf ihre Materie (Vergröberung der Körnigkeit) überarbeitet. So entstehen einige sehr schöne bildliche Momente, besonders in einer Passage, wo Merilee unter einem Wasserfall badet: eine Abfolge von Standbildern macht den Faden von Wassertropfen sichtbar und zeigt gleichzeitig den Willen der jungen Frau, sich angesichts dieser überwältigenden Kraft nicht unterkriegen zu lassen. Erst am Ende dieser langen Arbeit, die ein wenig wie eine Folter (des Films, von sich selbst und vom Vater) ist, wird Merilee ihrem Vater sagen können: „Ich liebe Sie“.

A SONG OF AIR ist ein typisches Beispiel für das, was man als „Filme der Rechnungsbegleichung durch den Familienfilm“ bezeichnen könnte. Solche Filme sind sehr zahlreich und überall auf der Welt zu finden, vor allem in Abschlussarbeiten an Kunsthochschulen oder Universitäten (Studenten schneiden ihre Familienfilme neu); man könnte fast sagen, dass es ein Genre geworden ist. 
Der Kontext, in dem diese Filme erscheinen, ist fast immer derselbe. Nach einer äußerst restriktiven Familienordnung (soziopsychologische Bedingungen) emanzipiert sich die junge Frau (oder der junge Mann), sucht ihren Weg und beginnt ein Leben, das dem von ihrer Familie vorgesehenen entgegengesetzt ist: Wir befinden uns im Raum der (Identitäts- und) Persönlichkeitsbildung. Diese Reise ist der Operator, der es dem jungen Menschen erlaubt, sich vom Familienfilmeffekt zu distanzieren (der oft eines der erlittenen Ordnungsinstrumente war) und zu diesen Filmen in einem intimen Modus zurückzukehren, aber mit einer kritischen Sichtweise. Nicht nur die vorherrschende Euphorie der Familienfilme wird als Unwahrheit angeprangert, auch Familienfilme erscheinen in dieser Lektüre als eindrucksvolle Operatoren der Opposition: Alles, was ein Faktor der Erfüllung und Garantie des Glücks war, wird nun als zerstörerisch angesehen.

Andererseits bringt die Suche nach einem Leben, das dem von der Familie vorherbestimmten entgegengesetzt ist, die junge Frau oder den jungen Mann dazu, sich dem Raum der Kunst zuzuwenden: Dort findet die Begegnung mit dem Kino statt (Merilee Bennett zum Beispiel absolvierte eine Ausbildung zur Fotografin und Filmemacherin am Philip Institute of Technology in Brisbane). Der Raum der Kunst ist besonders gut an die psychologischen Probleme angepasst, die diese jungen Menschen zu lösen haben: Es ist ein Raum, in dem sie den Platz des Autors und damit den des für die Produktion verantwortlichen Subjekts einnehmen können, d.h. einen Raum, in dem sie ihre Identität bestätigen können. Wie also kann man seine Rechnungen mit der Vergangenheit besser begleichen, als wenn man die Macht über die Filme seines Vaters übernimmt und dessen Familienfilme als Material für sein persönliches Schaffen verwendet? Der Operator ist die Arbeit des künstlerischen Schaffens: Bezeichnenderweise beginnt und endet A SONG OF AIR mit Aufnahmen von Merilee am Schneidetisch, wo sie die $16 \mathrm{~mm}$-Filme ihres Vaters bearbeitet. Es geht allerdings nicht wie bei A BARTOS CSALÁD darum, auf eine Metaebene überzugehen; der Eingriff in die Familienfilme ist hier radikaler: Er lässt sich als eine Operation der Zerstörung der Familienfilme des Vaters beschreiben, gefolgt von einer Arbeit der Wiederaneignung, die darauf abzielt, diese Filme in ein persönliches Werk zu verwandeln. 
Schließlich bedeutet die Migration von Familienfilmen in den Kunstraum, sie in den öffentlichen Raum (vs. den privaten Raum der Familie) zu bringen, was nicht nur ein starker Akt der Emanzipation ist, sondern auch eine offensichtliche Forderung nach Anerkennung: In diesem Raum werden Filme im künstlerischen Modus, d.h. in Bezug auf ihren Autor, gelesen (A SONG OF AIR ist ein Film von Merilee Bennett). Was den produzierten Text betrifft, so hat dieser einen doppelten Status: Einerseits ist er ein Werk, ein Film, der als zum Kunstraum gehörend anerkannt wird, und andererseits ist er ein Akt der Identitätsbestätigung. Wir befinden uns an der Schnittstelle zwischen dem Raum der Kunst und dem der Persönlichkeitsbildung.

Aber es gibt noch einen anderen Weg für Familienfilme, in den Kunstraum zu migrieren: Künstlerinnen und Künstler konzipieren ihre Familienfilme sofort als Teil dieses Raumes. Stan Brakhage und Jonas Mekas gehören zweifellos zu den Initiatoren dieser Bewegung, aber die Künstler, die in dieser Perspektive arbeiten, sind äußerst zahlreich. Die Titel zeigen deutlich die Beziehung zum Familienfilm auf: OH! My Mother (J 1969) und The Sons (J 1973) von Kohei Ando, ThE FAmily Album (Alan Berliner, USA 1986), DeR FATER (Christine Noll Brinckmann, BRD 1986), FAMILY PoRTRAIT (John Porter, CAN 1983) usw.; manchmal ist der Name noch einfacher und deutlicher: HOME MoviE (Titel von Filmen von Vito Acconci, Jane Oxenberg, Lee Ann Braown, Taylor Mead und anderen).

Man kann sich fragen, ob es legitim ist, hier von Migration $\mathrm{zu}$ sprechen, sofern es keinen Kontextwechsel gibt, sondern direkt eine Einschreibung in einen anderen Kontext: die Kunst. Mir scheint es jedoch, dass wir es mit einem Fall von Migration zu tun haben, denn der Familienfilm ist, wie sein Name schon sagt, ein Genre, das einem Kommunikationsraum zugeordnet ist: der Familie. Diese Art der Produktion in den Kunstraum $\mathrm{zu}$ bringen, ist daher eine echte Verschiebung. Vielleicht könnten wir von einer mentalen Migration sprechen, denn zunächst findet diese Verschiebung im Kopf des Regisseurs statt. Was den Zuschauer betrifft, so ist es die gegenteilige Verschiebung, zu der er eingeladen wird: Angesichts einer Produktion, die dem Kunstraum anzugehören scheint, ist er gezwungen, ihren Ursprung im Familienraum zu erkennen. 
Der Raum der Kunst überlagert sich also mit dem Raum der Familie, aber diese Überlagerung ist weder eine Fusion, noch eine kritische Metabeziehung (wie in A BARTOS CSALÁD), noch eine Beziehung der Wiederaneignungszerstörung (wie im Fall von A SoNG OF AIR). Es ist vielmehr ein Dominanzverhältnis: Der Raum der Kunst zwingt sich dem Familienraum auf. Wenn sie auch Familienfilme bleiben, so sind sie doch dafür gemacht, im künstlerischen Modus von einem Publikum außerhalb der Familie gelesen zu werden, behaupten ihre Regisseure, Autoren (Künstler) zu sein und verlangen, als solche anerkannt zu werden.

In diesen Filmen, wie auch im Familienfilm, finden sich viele Figurationen des „schlecht Gemachten“, aber, und das ändert alles, diese Figurationen sind hier freiwillig. In diesem Kontext werden diese Figurationen zur Marke des Autors (man erkennt einen Mekas-Film sofort an der Art, wie er mit Bildsprüngen spielt) und werden im ästhetischen Modus gelesen (der Betrachter freut sich über die plastische Dimension dieser verschwommenen, überbelichteten, grobkörnigen oder abgehackten Bilder). Es ist auch notwendig zu sehen, dass diese Figurationen, um zu funktionieren, Zuschauer erfordern, die bereit sind, sie in diesem Modus zu lesen. Das setzt voraus, dass diese Zuschauer derselben „Interpretationsgemeinschaft“ angehören wie der Regisseur (Fish 1980; Allard 1995), d.h. dass sie Teil desselben Raumes der ästhetischen Kommunikation sind.

Es ist also der ästhetische Raum, in dem diese Lektüre fußt, der es diesen Filmen erlaubt, in den Raum der Kunst einzutreten und im künstlerischen Modus gelesen zu werden; ohne diese Einschreibung der Aktanten der Kommunikation im selben ästhetischen Raum werden sie schlicht und einfach abgelehnt.

\section{Der Familienfilm im medizinischen Kontext}

Ich werde diese rasche Wanderung durch einige Kontexte, in die Familienproduktionen eintreten, abschließen, indem ich mich auf einen Kontext beziehe, der sich von allen, von denen ich bisher gesprochen habe, stark unterscheidet: den Kontext der medizinischen Forschung. 
Das erste Beispiel bezieht sich auf die Forschung in der Entwicklungspsychopathologie. Psychologen bitten Eltern von autistischen Kindern, ihnen ihre Familienfilme zu leihen, um zu versuchen, die Hinweise, die frühen Anzeichen, die eine frühzeitige Diagnose des Autismus ermöglichen würden, zu identifizieren um so effektiver in seine Behandlung einzugreifen (Adrien/Gattegno 2004). Die Untersuchung erstreckt sich auf Babys von der Geburt bis zum Alter von 18 Monaten. Ziel ist es, Anzeichen eines „Beziehungsrückzugs“ zu identifizieren: Kinder mit tiefgreifenden Entwicklungsstörungen hatten, verglichen mit normalen Babys, in den ersten Lebensmonaten höhere und nachhaltigere Rückzugswerte. Dies ist die Achse der Relevanz, die die Lektüre dieser Filme in diesem Kontext bestimmt. Die Lektüre wird hier von Wissenschaftler-Aktanten geleistet - wir befinden uns im Raum der wissenschaftlichen Forschung -, Aktanten, die einen ganz besonderen Operator mobilisieren: eine Ansammlung von hochspezialisiertem Wissen, ohne das wir nicht einmal wüssten, auf was wir bezüglich der gewählten Relevanzkriterien achten sollten. Der Modus der Sinnproduktion ist der dokumentarisierende Modus. Der Familienfilm wird zu einem Ersatzlabor. Während es für die Forscher unmöglich ist, selbst in die Familien zu gehen, um das Verhalten der Kinder zu studieren, macht der Familienfilm diese Forschung möglich: Einen Familienfilm zu sehen, ist ein bisschen wie das Leben mit dieser Familie während der Monate, in denen der Film gedreht wurde. Im Gegensatz zu dem, was wir in A BARTOS CSALÁD hatten, wird der Familienfilm als Medium ausgelöscht: Er gilt als transparent und gibt einen direkten Zugang zu den dokumentierten Zeichen des Autismus. Der Enunziator, der die dokumentarisierende Lektüre in Frage stellt, ist nicht der Film, sondern die Familie selbst.

Andere Ärzte nutzen den Familienfilm, um Patienten mit schweren Gedächtnisproblemen zu helfen. Wir befinden uns im Raum der Therapie. Jean-Claude Leners (2009) berichtet, wie im GerontologieZentrum von Pontalize (Luxemburg) „Reminiszenzsitzungen“ für Patienten mit Korsakoff-Syndrom organisiert werden, bei denen Ausschnitte aus Familienfilmen gezeigt werden. Diese Ausschnitte sind nicht unbedingt solche aus Familienfilmen der Patienten; sie können 
aus jeder Familie stammen. Die Sitzung findet einmal pro Woche statt. Der Kommunikationsoperator besteht aus Mikrosequenzen von ein bis zwei Minuten, die zu Themen ausgewählt werden, die starken Momenten im Leben entsprechen (Geburt, Heirat, Schule, Arbeit, lokale Traditionen usw.), d. h. Momenten, die jeder Patient wiedererkennen kann. Hier ist der Einsatz des Familienfilms ganz besonders relevant; sein stereotypisierter Charakter ist ein unschätzbarer Vorteil, da die Bilder umso wahrscheinlicher bei den Patienten auf Resonanz stoßen. Diese Sequenzen werden dann entweder allein oder in Serie (zum selben Thema) projiziert. Für diese Projektionen werden die Sequenzen so gewählt, dass sie sich auf den äußeren Kontext beziehen. Zum Beispiel, wenn die Vorführung im Dezember stattfindet, werden weihnachtliche Aktivitäten hervorgehoben; im Juli wird der Schwerpunkt auf Bilder aus den Sommerferien gelegt etc. Die kontextuellen Bedingungen verstärken also die Kraft der projizierten Bilder oder erzeugen zumindest eine Resonanz mit ihnen, um ihre stimulierende Kraft zu erhöhen. Die Patienten schauen diese Sequenzen in Gruppen in einem Krankenhauszimmer: Die Idee ist es, den intimen Modus der Sinnesproduktion durch den privaten Modus zu stimulieren. Nach jeder Vorführung werden die Sequenzen gemeinsam besprochen, aber die Patienten werden ermutigt, ihre intimste Erinnerung wachzurufen; Gruppendiskussion und Kontext befördern den Prozess des SichWieder-Erinnerns. Langfristiges Ziel ist es, den Gruppenmitgliedern ein stabileres Lebensumfeld zu ermöglichen. Schließlich werden alle Sitzungen transkribiert, so dass die Therapeuten die in anderen Sitzungen gewonnenen Informationen nutzen können. Der produzierte Text ist also doppelt: zum einen durch die Geschichten der Patienten, zum anderen durch die Transkriptionen, die ihrerseits als Operatoren dienen. Aber jenseits dieser Textproduktion liegt das Wesentliche in der Handlung am und durch den Patienten. Die Rolle des Familienfilms ist hier explizit performativ: Menschen dazu $\mathrm{zu}$ bringen $\mathrm{zu}$ reagieren, um sie zu heilen.

Diese Analysen zeigen die große Vielfalt der Kontexte, in die eine Produktion migrieren kann, sowie die Komplexität der Konstruktionen, die für ihre Einschreibung in diese verschiedenen Kontexte notwendig sind. Es ist jedoch möglich, die Vorgehensweise zusammen- 
zufassen (diese wäre genau so gewesen, wenn ich nicht wie hier die Migration einer Produktionsart untersucht hätte, sondern die Migration einer einzelnen Produktion: eines Films, einer Fotografie, eines Gemäldes, eines Textes, eines Musikstücks usw.).

In Bezug auf das vorige Kapitel, das sich auf eine einzige Achse der Kommunikation beschränkte, die a priori zu Beginn der Analyse festgelegt wurde, erfordert die Analyse der Migration einer Produktion in verschiedenen Kontexten, dass wir uns zunächst die Frage nach dem Raum oder den Räumen der Kommunikation stellen, die wir konstruieren müssen, um das Funktionieren dieser Produktion in diesen Kontexten zu erklären. Welcher Kommunikationsraum ist bzw. welche Kommunikationsräume sind für das Verständnis des Geschehens am relevantesten? Wie viele Kommunikationsräume müssen konstruiert werden? Wir haben zum Beispiel gesehen, dass, während ich für die Archive zwei Kommunikationsräume konstruiert habe, die den beiden einbezogenen Modi entsprechen, es mir, um die Verwendung der Familienfilme als Dokument im Fernsehen zu erklären, günstiger erschien, nur einen zu konstruieren, und das, obwohl man es doch mit zwei Modi zu tun hat. Das sind strategische Entscheidungen, die der Analytiker treffen muss, je nachdem, was ihm am geeignetsten erscheint, um die wichtigen Punkte der analysierten Kommunikationserfahrung so klar wie möglich darzustellen.

Sobald der Kommunikationsraum oder die Kommunikationsräume festgelegt ist/sind, erfolgt die Konstruktion der Aktanten und Kommunikationsoperatoren wie im vorigen Kapitel. Wenn mehrere Kommunikationsräume konstruiert wurden, stellt sich als nächstes die Frage nach der Beziehung, die zwischen diesen Räumen besteht: Die Analysen in diesem Kapitel haben die Beziehungen von Schnittpunkt, Übergang zur Metaebene, Überlagerung und Dominanz hervorgehoben. Das nächste Kapitel wird zeigen, dass noch weitere Beziehungen möglich sind.

Es bleibt noch zu fragen, was vom ursprünglichen Raum im neuen Kontext übrigbleibt, welche Rolle dieser Verweis auf den Ursprung spielt und welche Auswirkungen er hat. Man kann dann versuchen darzustellen, was aus der Produktion im neuen Kontext wird. Welchen Status bekommt sie? Bei Familienproduktionen reicht dies vom 
Dokument über die Reduktion auf eine Reihe von Gags oder auf Zeichen von Autismus (mit Auslöschung des Mediums), über ihre Positionierung als Analyseobjekt (A BARTOS CSALÁD) oder ihre Zerstörung/Rekonstruktion (A SONG OF AIR). Was ist ihre Rolle? Auch hier zeigen die Analysen die Vielfalt der Antworten: Die Rollen können informativ, relational, ideologisch, identitätsstiftend, therapeutisch etc. sein.

Schließlich ist es notwendig, nach dem Warum der Migrationen selbst zu fragen. Denn sie geschehen nicht ohne Grund und sind nicht unschuldig. So hat die Verbreitung von Familienfilmen heute an der Existenz eines riesigen Kommunikationsraums teil, der von starker Identität und gemeinschaftlichen Versuchungen, aber auch von einem Wandel in den Beziehungen zwischen Intimität, Privatheit und Öffentlichkeit durchzogen ist. Insgesamt sind diese Migrationen sowohl eine der Auswirkungen der Bedingungen dieses Raums als auch einer seiner Operatoren: Sie tragen auf ihrer Ebene zur Stärkung und Ausbreitung bei. 


\section{Kapitel 6}

\section{Textuelle Analyse \\ und Semiopragmatik}

Das Ziel der Semiopragmatik besteht darin, „den immanentistischen Ansatz in die kontextuell pragmatische Perspektive zu stellen. Wenn man die kontextuellen Bedingungen der Textkonstruktion anerkennt, kann auch die immanentistische Analyse angewandt werden“. So lautete die Definition des semiopragmatischen Programms im einleitenden Kapitel. Die verwendete Formulierung könnte vermuten lassen, die pragmatische Perspektive ändere nichts an der textuellen Analyse, so wie sie unter dem immanentistischen Paradigma praktiziert wurde. Dies ist aber nicht der Fall. Auch wenn es stimmt, dass die Werkzeuge der immanentistischen Analyse sehr nützlich bleiben, so erleben wir doch eine radikale Transformation der textuellen Analyse: Wie wir bereits durch die Analysen in den beiden letzten Kapiteln feststellen konnten, geht es von nun an nicht mehr darum, einen existierenden Text zu analysieren, sondern die Erfahrung der Arbeit einer textuellen Produktion in ihrem Kontext.

Ich möchte nun auf die Bedingungen eingehen, die sich am meisten der traditionellen textuellen Analyse annähern: die Analyse einer spezifischen Produktion in einem einzigen Kontext. Ich hoffe, dass die Unterschiede dadurch noch deutlicher werden.

Die drei Produktionen, die ich analysieren werde, wurden aufgrund der Vielfältigkeit der Felder gewählt, in denen sie vorkommen: Die Fernsehübertragung einer Etappe der Tour de France, diesem mythischen Moment des Radsports; der Nachdruck eines Gemäldes in einem kunsthistorischen Werk; eine von einer Gruppe durchgeführte Forschungsarbeit zu einem Film an der Universität.

Der Ablauf ist folgender: Ich beginne mit der Beschreibung des Kontextes, in dem die Lektüre stattgefunden hat, dann werde ich den Begriff des Kommunikationsraums heranziehen, um das kommunika- 
tive Funktionieren dieser Lektüre zu charakterisieren sowie die Erfahrung, die diese auslöst. Das Ziel besteht darin, diese Lektüre in dem definierten Kontext in all ihren Dimensionen aufzuschlüsseln (oder zumindest so weit wie möglich).

\section{Eine Etappe der Tour de France im Fernsehen}

Es ist schwer, dem Druck dieses „totalen [...] Mythos“ der Tour de France zu widerstehen (Barthes 2010 [1957], 152f). Durch Presse, Funk und Fernsehen verbreitet, lädt er dazu ein, drei Wochen lang „das eigene Leben mit dem der Tour zusammenzuführen“ (Arnaud/ Chandelier 2000, 250). Philippe Gaboriau $(1995,16)$ sah darin „einen Ersatz für den Befreiungswunsch des Kleinbürgermilieus“, doch die Einladung betrifft noch weitergehend alle sozialen Schichten mit einer weiten Geste der Versöhnung mit der Welt. Auch wenn ich kein großer Fan von Sportübertragungen bin, habe ich mich dennoch dafür entschieden, mir die 17. Etappe der Ausgabe von 2010 anzuschauen, eine entscheidende Etappe, denn es müssen mehrere Gebirgspässe überwunden werden: der Marie-Blanque, der Soulor und vor allem der mythische Tourmalet. Die Tatsache, dass ich die Region kenne, weil ich im Urlaub durchgefahren bin, war wahrscheinlich nicht ganz unschuldig an meiner Entscheidung; meine Erwartung ist also ebenso touristischer wie sportlicher Natur.

In dem Café, in dem ich mich niedergelassen habe, herrscht Feststimmung: Die Tour de France anzugucken, verleitet zu Euphorie und Geselligkeit. Von einem Tisch zum nächsten fällt man sich ins Wort, erinnert man an die letzte Etappe. Die Tour de France ist eine Fernsehserie: Was ich heute sehen werde, ist davon abhängig, was gestern passiert ist, vorgestern und die Tage davor. Nach und nach fügt sich die gesamte Geschichte der Tour zusammen. Wenn es eine Sendung gibt, die auf einem vorherigen Wissen basiert, dann ist es diese hier. Ich muss gestehen, dass mir viel von diesem Wissen fehlt, aber die Stimmung ist ansteckend und ich lasse mich auf das Spiel ein: Sogar wenn ich mich absolut nicht in der Materie auskenne, kann ich mich in die Unterhaltung einbringen. Die soziale Dimension der Kommunikation kann auf der Ebene der Sinnproduktion manchmal erstaunliche 
Dinge bewirken ... Auf der anderen Seite stellt die Übertragung der Tour de France eine abgewandelte Form dessen dar, was Daniel Dayan und Elihu Katz (1992) „zeremonielles Fernsehen“ genannt haben: Sie bewirkt eine „spezifische Geselligkeit“, „ein Verlangen nach Gemeinschaft“, „das überwältigende Gefühl“ in eine unendlich große, grenzenlose Zuschauermenge einzutauchen (Dayan/Katz 1992, 197 u. 206). Um eine Formulierung aufzugreifen, die ich verwendet habe, um den Fiktionseffekt zu beschreiben, ließe sich hier von „Mise en phase“ sprechen, aber es geht nicht darum, im Rhythmus der erzählten Ereignisse mitzugehen (die Übertragung hat noch nicht angefangen): Das Caféhaus schwingt mit den Millionen Zuschauer mit, die sich darauf vorbereiten, alle zur selben Zeit die Tour zu gucken, also: spektatorielle Mise en phase.

Die Übertragung übernimmt France 3. Der Titel der Sendung, „En direct du tour“ („Live von der Tour“), bestimmt die Achse der Relevanz, auf der ich eingeladen bin mich einzuschreiben, an der Schnittstelle zwischen dem Kommunikationsraum des Sports und dem der Liveübertragung: Was ich sehe, geschieht in dem Moment, in dem ich es sehe. Weit davon entfernt, die Vermittlung zu leugnen, drängt mich die Liveübertragung dazu sie wahrzunehmen: Es ist der Sender France 3, der die Tour zeigt, er ist der Enunziator, ein realer Enunziator, hinterfragbar in Bezug auf Identität (worin bestehen die Unterschiede zwischen den Sportübertragungen von France 3 und denen anderer Sender?), Wahrheit (ich hoffe, dass alles, was man mir über die Tour sagt, wahr ist; der Sender setzt im Übrigen alles daran, um mich davon zu überzeugen: Rückgriff auf Experten, auf ehemalige Rennradfahrer usw.) und Tun: Welcher ist der Operator der Kommunikation? Wie funktioniert er?

Der Operator der Kommunikation kann als technisches Dispositiv der TV-Liveübertragung bezeichnet werden, und ich muss sagen, dass er manchmal zu wünschen übrig lässt: Regelmäßig bleibt das Bild stehen, die Fahrer werden in ihrem Schwung gebremst; es kommt auch vor, dass das Bild springt. Paradoxerweise haben diese Probleme einen positiven Effekt: Sie verstärken den Liveeindruck. Die Kommentatoren zögern im Übrigen nicht, davon zu sprechen: Es handelt sich um „Risiken der Liveübertragung“. Der Kommentar bezieht sich auch 
oft auf dieses Dispositiv: auf die Rolle der Hubschrauber, auf Passagen mit Aufnahmen vom Motorrad aus, auf die Betonung der Organisation, die all dies braucht, auf die technische Komplexität. Diese (reflexive) Meta-Dimension unterstreicht die Bedeutung des technischen Operators und zugleich eines Operators, der dazu in der Lage ist, seine Arbeit zu beurteilen: Die menschliche Dimension der Kommunikation wird verstärkt.

Als Experte (Berufskrankheit) kann ich nicht umhin anzumerken, dass die Montage allzu oft die „180-Regel“ bricht: Die Rennradfahrer durchkreuzen den Fernsehbildschirm von rechts nach links, dann in der nächsten Einstellung von links nach rechts. Dies ändert jedoch nichts an meiner Wahrnehmung des Rennens: Mir käme nicht in den Sinn, dass die Fahrer umdrehen, denn ich weiß (durch eine aus einem kognitiven Schemata hervorgegangene Bedingung), dass das Rennen linear verläuft: „Der Raum entspricht einem Vektor und die „Bedingungen sind vom Typ „,davor sein', ,dahinter‘, ,nah‘, ,weit““ (Colin 1990, 95). Wie Colin anmerkt: „Das Wissen, das der Zuschauer aus der audiovisuellen ,Sprache‘ erworben hat, erscheint nicht ausreichend, um räumliche Beziehungen im Rahmen des Fahrradrennens“ zu verstehen (ibid., 103); davor muss man wissen, dass es sich um ein Radrennen handelt. Einen Moment lang ist meine Haltung zwiespältig: Meine Erfahrung als Filmwissenschaftler hinterfragt meine Erfahrung als Zuschauer, aber der Zuschauer siegt.

Von den ersten Einstellungen an lässt mich France 3 in die Welt der Tour eintauchen (Diegetisierung): Nach einer Supertotalen aus dem Hubschrauber auf die Spitzengruppe zeigt mir eine Einstellung den Point-of-View der Zuschauer, dann platziert mich eine Subjektive von einem Motorrad aus quasi an die Stelle der Fahrer: Ich bin mitten in der Spitzengruppe. Das Rennen selbst erscheint wie eine große Erzählung, unterteilt in eine Aufeinanderfolge von Minierzählungen, jede mit ihren eigenen Herausforderungen (vor allem die Passüberquerungen). Die Tour anzuschauen heißt, sich in eine stark narrative Erfahrung hineinzubegeben: Wer wird siegen? Die Programmvorschau versprach, mich nicht nur am Rennen teilhaben zu lassen, sondern „die Tour auch mitzuerleben“: Sie hat nicht gelogen. Die Mise en phase, dieses Mal die narrative, kommt voll zum Tragen. 
Auch wenn ich drei Prozesse umsetze, die man in der Fiktionalisierung wiederfindet (in eine Welt eintauchen, eine Erzählung verfolgen, mit den erzählten Ereignissen mitgehen), ist meine Lektüre keine fiktionalisierende: France 3 wird als realer Enunziator konstruiert. Meine Lektüre löst zwei Modi aus: den Modus des Spektakels und den dokumentarisierenden Modus, in beiden Modi kommt die Narration vor. Ich bin zugleich Zuschauer und Lernender (jemand, der sich die nötigen Informationen wünscht, um das Rennen zu verstehen). Auf der dokumentarischen Ebene begnügt sich die Sendung nicht damit, mich über den Ablauf des Rennens zu informieren, sie bietet mir den „Diskurs“ der Erzählung an: Der Off-Kommentar nennt die Fahrer, erklärt die Strategien der Mannschaften, gibt technische Details etc. Für mich, der ich mich nicht auskenne, sind diese Informationen wertvoll: Sie ermöglichen mir zu verstehen, was im Rennen vor sich geht. Auf der Ebene des Spektakels werde ich bedient: Es gibt zunächst die Kulisse, die die Hubschrauberaufnahmen mich entdecken lassen, eine durch Wolkenwanderungen belebte, prächtige Bergkulisse. Und dann ist die Etappe durchaus reich an Ereignissen: wolkenbruchartige Regenfälle, die auf die Fahrer niederprasseln, der Sturz von Samuel Sanchez (wird der angeschlagene Fahrer weiterradeln oder nicht?), das Duell Alberto Contador vs. Andy Schleck, die schöne Geste des gelben Trikotträgers, den Sieg dieser Etappe seinem Rivalen zu überlassen ... All dies bringt mich dazu, einen, wie ich sagen würde, sportlichen Text zu konstruieren, der dokumentarisch und spektakulär zugleich ist.

Zeitweise tritt die Übertragung der Tour zurück zugunsten des Kommunikationsraums der Werbung: Ich verlasse die Live-Übertragung, behalte aber den dokumentarisierenden Modus bei, die Operatoren sind jedoch Werbefilme. Die Verbindung zum Raum des Sports wird allerdings nicht verlassen, denn die Filme benutzen diesen oftmals, um daraus ihre Mini-Szenarien zu bilden: Beide Räume durchkreuzen einander. Das amüsiert mich sehr und ich bin ganz schön beeindruckt von den Produzenten, die die Situation so gut auszunutzen verstanden haben (ästhetischer Modus). Jetzt konstruiere ich jedoch keinen sportlichen Text mehr, sondern eine Reihe von Werbetexten, deren Ziel es ist, mich aus meiner Position als Zuschauer 
herauszulocken, um, indem mit dem Ansehen der Tour und ihrer Fahrer gespielt wird, aus mir einen Konsumenten zu machen und mich zum Kaufakt zu verleiten. Ich bezweifle, dass es klappt, aber man weiß ja nie ...

Die Übertragung lässt mich schließlich den touristischen Kommunikationsraum betreten, den ich von Anfang an erwartet hatte. Geschickt präsentiert sich der touristische Teil, als wäre er in den selben Kommunikationsraum eingeschrieben wie das Rennen (einschließende Verbindung; man verlässt nie den sportlichen Raum): Der Hubschrauber zeigt mir die Fahrer, wie sie das Dorf Oloron durchfahren, überfliegt dann die Kathedrale Sainte-Marie d'Oloron. Die folgenden Einstellungen zeigen ausführlich das Portal und lassen mich die Kathedrale selbst betreten. Diese letzten Bilder sind sicherlich vorab gedreht worden, aber der Live-Effekt wirkt weiter. Ich bin nun in einen Touristen verwandelt: Es ist ein bisschen so, als würde ich wirklich Oloron besuchen. Manchmal ist der Fernsehbildschirm zweigeteilt (Splitscreen): Links der touristische Dokumentarfilm, rechts geht das Rennen weiter, wodurch die gleichzeitige Anwesenheit beider Kommunikationsräume visuell zum Ausdruck gebracht wird. Das Ergebnis von alldem ist die Konstruktion eines dritten Textes: der touristische Text.

Aber meine Lektüre endet nicht hier: Während meines Urlaubs in den Pyrenäen bin ich durch Oloron gekommen. Die Kleinstadt wird also zu einem Kommunikationsoperator, der mich in den Raum der Erinnerung eintauchen lässt. Ich erinnere mich: Mein Auto hatte ein Problem und ich musste in der Stadt wenden, wütend darüber, Zeit verloren und einige Urlaubsstunden mit der Suche nach einer Werkstatt verschwendet $\mathrm{zu}$ haben (Modus der intimen Sinnproduktion). Dann habe ich die Kathedrale besucht. Ich war bestrebt, die Architektur gut zu verstehen (künstlerischer Modus) und hatte aufmerksam gelesen, was im Reiseführer dazu stand. Ich erinnere mich gut an das Gefühl, dass ich beim Anblick des Portals und der Kapitellen empfand; ich schaute sie lange an (ästhetischer Modus). Dieser erzwungene Stopp hat schließlich zu einer ganz interessanten Erfahrung geführt: die Produktion eines persönlichen Textes kombiniert mit dem intimen Modus, künstlerischen Bezügen und einem 
ästhetischen Gefühl. Während ein paar Momenten habe ich quasi das Rennen vergessen: Ich muss jetzt den Faden der sportlichen Erzählung wieder aufnehmen ...

\section{Lektüre der Reproduktion eines Gemäldes}

Ich habe die vorherige Analyse absichtlich mit einem persönlichen Touch versehen. Daraus sollte man aber nicht ableiten, dass die semiopragmatische Textanalyse einzig der Beschreibung der individuellen Erfahrung dient.

Exemplarisch werde ich nun die durch den Band Renaissance in Venedig von Patricia Fortini Brown bestimmte Lektüre eines Gemäldes von Tizian, Die Familie Vendramin vor der Kreuzreliquie (1543-1547) beschreiben. Ich betrachte den Band von Brown in diesem Zusammenhang als den Kontext, der die Lektüre des Gemäldes von Tizian regelt und das, was ich zu beschreiben suche, ist die Erfahrung des Lesers dieses Bandes und nicht bloß meine persönliche Erfahrung.

Der Band Renaissance in Venedig gehört zur Kunstband-Sammlung publiziert bei DuMont. ${ }^{1}$ Diese einfache Erwähnung verpflichtet dazu, den Kontext zu erweitern; alle Bestimmungen des Raums der Kunst werden im Band von Brown transportiert: Der Leser wird angewiesen, mit dem Eigennamen (dem Autorennamen) $\mathrm{zu}$ arbeiten, sich für biografische, thematische, stilistische, historische Fragen etc. zu interessieren.

Die Sammlung enthält diverse Unterkategorien: Grammatik der Stile, Geschichte, Monographie, Enzyklopädie. Der Band zur Renaissance in Venedig gehört zur Reihe art in context, in der man ebenso Renaissance in Florenz, Eine Welt im Umbruch, Impressionismus, Der fotografierte Körper, Die Kelten etc. findet. Im Vorwort zu der Reihe heißt es: „Dies ist die Einführungsreihe und Gesamtübersicht schlechthin, ausrichtet auf die Darlegung der historischen, ökonomischen,

1 [Anm. d. Ü.:] Der DuMont Verlag hat die Sammlung von Flammarion ins Deutsche übersetzt herausgegeben, allerdings gibt es hier keinen übergeordneten Titel für diese (Kunstband-)Sammlung und somit auch nicht die von Odin genannten Unterkategorien, sondern nur die Reihe „art in context“. Die von Odin genannten Titel entsprechen dieser Reihe. 
politischen und kulturellen Bedingungen künstlerischen Schaffens“. ${ }^{2}$ All dies verleitet dazu, diese Definition als Achse der Relevanz anzusehen, von der aus sich der Kommunikationsraum bildet, in welchem der Leser operieren soll. Ich spreche hier von einem „diskursiven“ Kommunikationsraum, ein Raum, der aus dem Rezipienten einen engagierten Leser mit einer „diskursiven“ Erfahrung macht.

Der Kommunikationsoperator setzt sich aus einer Mischung von Text und Bild zusammen (Karten, Gemäldenachdrucke, Fotos von Denkmälern, Gebäuden, Skulpturen, Plätzen in Venedig ...). Im Kapitel VI: „Stände, Schichten und Geschlechter“ und noch genauer auf der Seite 162 in einem Unterkapitel, das sich dem „Kult der Familie“ widmet, heißt es: „Die zentrale Bedeutung der männlichen Linie für das Patriziat findet beredten Ausdruck in Tizians Gemälde Die Familie Vendramin vor der Kreuzreliquie (Abb. 116)“. Der Verweis in Klammern lädt den Leser dazu ein, den Nachdruck des Gemäldes zu betrachten, der etwa ein Drittel der folgenden Seite einnimmt. Die Art, wie dieser Nachdruck eingefügt ist, eingezwängt in das Kleinformat des Textes, verleitet dazu, ihn als ein Element des „Diskurses“ über den Status der Familie im Venedig der 1500er Jahre zu lesen (dokumentarisierender Modus) und, noch genauer, als eine Illustration des „Diskurses“ über „die zentrale Bedeutung der männlichen Linie für das Patriziat“. Selbst wenn einige erwachsen und andere Kinder sind, so ist nur das männliche Geschlecht auf diesem Gemälde vertreten. Die wiedergegebene Szene zeigt, dass die Männer der Familie Vendramin eine starke Beziehung zur Religion hatten: Die Figuren drücken ihre Frömmigkeit einem Kreuz gegenüber aus, das sich im Gemälde rechts auf einem Altar befindet (Konstruktion eines dokumentarischen Textes).

Die Legende und Anmerkung zum Gemälde fungieren als Bedingungen mit dem Ziel, die Lektüre zu regeln, um zu verstehen, dass es sich um ein Gruppenfamilienbild handelt, das im Kommunikationsraum der Familienerinnerung der Familie Vendramin realisiert wurde. Tizians Gemälde gehört zur Reihe der Erinnerungsoperatoren,

2 [Anm. d. Ü.:] Dieses Zitat wurde aus dem der Reihe bei Flammarion übersetzt. Bei der Reihe im DuMont-Verlag existiert kein entsprechendes übergeordnetes Vorwort zu der Reihe. 
die im vorigen Kapitel untersucht wurden. Der Text von Brown nennt die unentbehrlichen Elemente, die dem Leser erlauben, in diesen Raum einzutreten und sich vorzustellen, wie dieses Gemälde in diesem Rahmen gelesen wurde (privater Modus): Vorstellung der Figuren („Hinter Andrea stehen sein ältester Sohn Leonardo, bereits ein bärtiger Zwanzigjähriger, als das Gemälde begonnen wurde, und Bartolo (15 Jahre), Francesco (14 Jahre) und Luca (13 Jahre) ...“); Einladung, das Gemälde wie ein fotografisches Gemälde zu lesen (in dem Sinn, den ich dem Ausdruck im vorigen Kapitel gegeben habe); Informationen über die Stellung der Figuren innerhalb der Familie (Andrea ist in Begleitung seiner sieben Söhne gemalt, aber nicht seiner sieben Töchter, die in Belegen erwähnt werden); Erzählung der Geschichte, die der Szene zugrunde liegt: Andrea Vendramin war im Jahr 1369 der Verantwortliche der Scuola di San Giovanni Evangelista - in diesem Jahr erhielt die Bruderschaft die Reliquien des Heiligen Kreuzes ... (Konstruktion eines familiären Textes).

Der Band gibt an, dass sich das Gemälde von Tizian als Operator für die Erinnerung der Familie Vendramin aufgrund des Bekanntheitsgrades des Malers eingebürgert hat (künstlerische Zuweisung), eine Bekanntheit, die eine Familie würdigt, die eines seiner Werke besitzt (der Raum der Familie durchkreuzt den Raum der Kunst). Gleichzeitig lenkt der Text den Leser dahingehend, sich in den Raum einzuschreiben und das Gemälde unter Verwendung des „vollen künstlerischen Modus“ zu betrachten: Konstruktion des Lesers als Kunstamateur. Brown betont, dass das Gemälde sich in London befindet, in der National Gallery, der Hochburg der Kunst, wenn man so will; dass es sich um eine Leinwand vom Format 210 x $300 \mathrm{~cm}$ handelt; und nennt dann Elemente, um die künstlerische Lektüre anzuleiten: Im Gegensatz zu dem, was man annehmen könnte, lenkt die Komposition von Tizian die Aufmerksamkeit weg vom Heiligen Kreuz (verbannt in die obere rechte Ecke des Gemäldes) und hin auf die Familienmitglieder. Nach Brown schafft der Tizian „eine meisterliche Lösung des Problems, nicht weniger als neun Figuren in einer Szene zu porträtieren und gleichzeitig deren individuellen Charakter zu bewahren“: Die traditionelle Formel der Prozession wird abgelehnt, die Figuren sind in Gruppen im Raum des Gemäldes verteilt. Es gibt 
zudem eine ganze Reihe von Kommentaren zum Farbeinsatz bei Tizian, ein Einsatz, der die „Bildfläche zu einer Einheit zusammen[fügt]“: Das helle Himmelblau, das Braun des Altars, zahlreiche in Schwarz gekleidete Figuren (Gabrielle und seine sechs jüngeren Neffen) bilden einen Untergrund, der den Kontrast mit den roten Gewändern der anderen Figuren erlaubt, Rottöne, die von Dunkelrot über Brillantrot bis Rosarot reichen ... (Konstruktion des Textes: Kunst von Tizian).

So wie Brown die Arbeit von Tizian lobt, könnte man meinen, sie wolle den Leser dazu bewegen, das Gemälde ebenfalls zu bewundern (Versuch der Konstruktion eines ästhetisierenden Leser-Aktanten), aber der Übergang zum ästhetischen Raum gelingt schlecht; auch wenn der Nachdruck von guter Qualität ist, so ist es doch schwierig, bei einem so kleinen Format $(13 \times 9 \mathrm{~cm})$ den ästhetischen Modus einwandfrei funktionieren zu lassen. Dies wäre vermutlich möglich, wenn das Gemälde ganzseitig abgedruckt wäre, aber das würde bedeuten, es in einen separaten Teil des Buchs zu überführen und dies würde die Hierarchie der Modi ändern: Die ästhetische Lektüre hätte die Oberhand, aber dann müsste man sich anstrengen, um das Gemälde mit dem „Diskurs“ des Textes in Einklang zu bringen und eine dokumentarisierende Lektüre zu ermöglichen (es braucht nicht viel, um die Hierarchie der Räume und Lektüremodi zu ändern). Der Band hat also die geeigneteste Lösung für sein „diskursives“ Ziel gewählt, auf die Gefahr hin, die ästhetische Erfahrung zu opfern.

\section{Eine kollektive Forschungsarbeit an der Universität}

Von 1969 bis 1972 haben drei Forscher, Claude Bailblé, Michel Marie und Marie-Claire Ropars, daran gearbeitet, eine Lektüre des Films MURIEL von Alain Resnais (F 1963) zu produzieren: eine Lektüre, die 1974 zur Publikation des Buchs Muriel, histoire d'une recherche geführt hat. Es handelt sich um die Erfahrung der Forscher, eine seltene Erfahrung, die es verdient, in Erinnerung gerufen zu werden; und ich werde nun versuchen, sie zu analysieren.

Der Kontext ist der eines universitären Forscherteams und die Bedingungen dieses Strukturtyps regeln die Erfahrung der Teilnehmer 
wie auch ihre Textproduktion. Mit dem „Vorwort“ wird die Achse der Relevanz definiert: „Als wir uns als Gruppe formierten, haben wir uns zum Ziel gesetzt, einen Film systematisch zu analysieren, indem die Kategorien ausgemacht werden sollten, die die Funktions- und die Bedeutungskonstruktionen regeln“ (Bailblé/Marie/Ropars 1974, 7). Wir befinden uns im Kommunikationsraum der Analyse. Genauer gesagt befinden wir uns an der Schnittstelle zwischen dem Raum der Analyse und dem der Universität. Diese Beziehung zum universitären Raum unterscheidet die Analyse von MURIEL von zahlreichen kritischen Analysen in Fachzeitschriften, die sich diesem Film widmen: Einerseits ist die Bedeutungsproduktion methodischen und wissenschaftlichen Bedingungen unterworfen, die dem Raum der Kritik nicht abverlangt werden; andererseits gehört der Kritiker dem Raum des Kinos an (indem er die Zuschauer dazu verleitet, sich diesen oder jenen Film anzusehen), wohingegen der Universitätsforscher außerhalb dieses Raums steht (der Untersuchungsgegenstand befindet sich nicht im „Feld“ Kino). Der spezifische Status des Universitätsforschers verleiht ihm eine besondere Kraft: die Macht des Außenstehenden.

In Bezug auf den üblichen Raum der Sichtung eines Films zeigt sich der Übergang in den Raum der universitären Analyse durch einen Zeitenwechsel: Im Fall von MuRIEL hat die Erfahrung der Lektüre drei Jahre gedauert, sicherlich nicht durchgängig, aber in einem zeitlichen Verhältnis, das nichts mehr mit der Projektionsdauer zu tun hat. Die Position des Aktanten ist ebenfalls nicht mehr dieselbe: Die Forscher sind keine Zuschauer, sondern universitäre Filmanalytiker. Das Problem wird zusätzlich dadurch erschwert, dass die Forscher auch Zuschauer sind (sie gehen ins Kino) und es steht fest, dass sie MurIEL, bevor sie ihn analysierten, als Zuschauer gesehen haben. Diese doppelte Aktantenrolle, die eine Überlagerung der beiden Kommunikationsräume bewirkt, des Raums des Zuschauers und desjenigen der Universität, stellt zugleich die notwendige Bedingung und eines der Hauptprobleme der Analyse dar (ich komme später darauf zurück). Im Raum der universitären Analyse ist der Kommunikationsoperator nicht mehr der Film, sondern das Ensemble an theoretischen und methodischen Werkzeugen, die zur Analyse beitragen. Der verwendete Modus der Sinnproduktion schließlich ist nicht mehr der des 
Spektakels oder der fiktionalisierende Modus, auch nicht der künstlerische oder ästhetische, sondern der analytische Modus.

Der analytische Modus ist eine Unterform des (bereits erwähnten) „diskursiven“ Modus; in der Tat, wenngleich nicht jede „Diskurs“Produktion die Analyse als vorrangiges Ziel hat (auch wenn sie oftmals unterschwellig angenommen wird), so stellt doch jede Analyse eine „Diskurs"-Produktion dar. Wie der ästhetische und der künstlerische Modus auch, so ist ebenso der analytische Modus horizontaler Natur: Er durchläuft mehrere Phasen und kann im Verlauf unterbrochen werden (eine Analyse kann oder kann nicht zu einem Ergebnis führen). Die Analysephasen hängen vor allem von der fachlichen Disziplin und der verwendeten Methode ab: In diesem Fall handelt es sich um den Rahmen der textuellen Filmanalyse.

Um die Analyse von MURIEL korrekt wiedergeben zu können, scheint es mir notwendig, diese Phasen als unterschiedliche Kommunikationsräume zu behandeln: Es handelt sich zwar sicherlich um Unterräume des Raums der Analyse, aber sie haben ihre eigenen Akteure, Operatoren und Ziele.

Der Raum der Beschreibung und die Konstruktion des Analyseobjekts

Die Forscher beginnen mit dem Auseinandernehmen des Films, einer découpage après montage, also einem Einstellungsprotokoll, das den projizierten Film wiedergibt. Der Forscher handelt hier als Beobachter. Auseinandernehmen [découper] heißt ausmachen und kategorisieren (oder kategorisieren und ausmachen). Das Ergebnis ist die Umwandlung des filmischen Texts in einen schriftlichen, einen Text in Form einer Tabelle, die nicht weniger als zwölf Spalten beinhaltet. Die Eingaben in der Betreffzeile sind die für den Schnitt notwendigen Operatoren: Einstellungsnummer, Dauer, Situation, Bewegung der Figuren (inklusive Blickrichtung), Farbe, Einstellungsgröße (Nahe, Halbnahe ...), Winkel, Kamerabewegung, Tonspur (Geräusche, Musik, Dialoge, Quelle des Tons [on/off]).

Daneben illustrieren 1300 Fotogramme jede einzelne Einstellung. Wenngleich es sich, wie Michel Marie schreibt (ibid., S. 335) „um 
fotografische Reproduktionen der originalen Fotogramme des Filmstreifens handelt", so kann man daraus nicht ableiten, dass die Reihe der Fotogramme eine Reproduktion von MuRIEL darstellt (nicht zu vergleichen, beispielsweise, mit dem Nachdruck des Tizian-Gemäldes im vorigen Beispiel). Man könnte eher sagen, es handele sich um eine Verdichtung des Films: MURIEL in 1300 Fotos. Im Unterschied zur Verdichtung des Bildhauers Armand ergibt sich die Verdichtung hier aus einem Entnahmeprozess: Ein Bild pro Einstellung wurde entnommen, nur komplexe Einstellungen werden in mehreren Fotogrammen wiedergegeben. ${ }^{3}$

Die erste Funktion dieses Ensembles (Einstellungsprotokoll [découpage] plus Fotogramme) dient dazu, den Forschern den Film präziser in Erinnerung $\mathrm{zu}$ rufen. Die Erinnerung eines Films ist natürlich etwas sehr labiles und das Risiko, mit Sequenzen zu arbeiten, die mehr oder weniger erfunden sind, ist nicht zu vernachlässigen. Bellour weist darauf hin, dass André Bazin beteuerte, „obgleich er das intellektuelle Gewissen schlechthin“ war, dass THE RIvER (DER STROM, Jean Renoir, F/GB/IND/USA 1951) ausschließlich aus festen Einstellungen bestehe, obwohl er über zwanzig Fahrten enthält und er selbst, als er BRIGADOON (Vincente Minnelli, USA 1954) analysierte, zwei feste Einstellungen in eine „bewundernswerte Kamerafahrt“ verwandelte, die er „buchstäblich erträumt“ hatte (Bellour 2001 [1979], 5). Fotogramme und Einstellungsprotokoll dienen dazu, derartige Probleme zu vermeiden, aber ihre Aufgabe geht darüber hinaus.

Es ist in der Tat nicht einfach, sich als Analyst gegenüber einem Film zu positionieren. Alle Filmwissenschaftler, die unterrichten, kennen die Schwierigkeit, von der Zuschauerposition zur Analytikerposition $\mathrm{zu}$ wechseln. Insbesondere wenn man selbst cinephil ist. Einzelbilder festhalten, eine découpage verfertigen (der Ausdruck [der die Konnotation des Zerschneidens aufruft; Anm. d.Ü.] selbst ist heftig), bedeutet, das Objekt der Liebe, das ein Film darstellt, sowie die Faszination dafür zu zerstören und eine für die Analyse nötige Distanz zu gewinnen (Metz 2000 [1975/1977]; Bellour 2001 [1979]).

3 Das vollständige Protokoll konnte nicht publiziert werden, im Werk ist aber im vierten Kapitel ein vereinfachtes Beispiel wiedergegeben (ibid., S. 248-261). 
Auf der anderen Seite wird die zeitliche Struktur des Films durch den Schnitt und die Einzelbilder in eine räumliche Struktur überführt (eine Tabelle, eine Bildreihe). Das Ziel für den Forscher besteht darin, seinen Lektüreweg wählen zu können: Er kann natürlich der Einstellungsfolge und auf diese Weise der Zeitlichkeit des Films folgen, er kann aber auch auf einem Einzelbild verharren - Bellour sieht in der Entscheidung für oder gegen ein „Standbild“ [arrêt sur image] ein distinktives Kriterium für die analytische Haltung (Bellour 2001 [1979], 2) - oder einen nichtlinearen Kurs einschlagen: Einstellung 5 mit den Einstellungen 75 und 122 vergleichen, von Einstellung 375 auf Einstellung 28 zurückkommen. Er kann auch „Hypothesen über die Gruppierung von Einstellungen zu Bedeutungseinheiten aufstellen“ (Bailblé/ Marie/Ropars 1974, 33), je nach Rang, gefilmten Figuren oder Rahmen, in dem sich die Szene abspielt (zwischen den Seiten 144 und 145 werden auf vier Seiten als Gruppe zusammengefasste Einzelbilder wiedergegeben, um die Modalitäten der Raumkonstruktion zu verstehen).

Das Einstellungsprotokoll kommt schließlich einer Beschreibung des Films gleich. Die meisten Parameter des Protokolls werden in der Regel nicht von den Zuschauern wahrgenommen: Der Zuschauer kümmert sich weder um die Anzahl der Einstellungen, noch um die Kadrierung, noch um die Kamerabewegungen ...; er ist von der Erzählung gefesselt, von der Fiktion, und wenn es Parameter gibt, die auf ihn einwirken, dann unbewusst. Das Auseinandernehmen bringt diese Parameter auf explizite Weise hervor. Was die Fotogramme betrifft, wenngleich diese auch selbst nicht beschreiben (sie zeigen), dann liefern sie, vorausgesetzt man hinterfragt sie, eine beachtliche Menge an Informationen, die das Einstellungsprotokoll nicht liefert: In diesem Sinne nehmen sie am Beschreibungsprozess teil.

Einstellungsprotokoll und Fotogramme führen $\mathrm{zu}$ einer anderen Sicht auf den Film: Sie bilden eine erste Analyseebene des Films, einen ersten Text, der einer ersten Lektüre von MuRIEL entspricht. Zugleich verhelfen sie $\mathrm{zu}$ einer weitergehenden Analyse, indem sie den Forscher-Zuschauern ermöglichen, sich als Analytiker zu positionieren, und aus dem Film ein Analyseobjekt machen. 
Vom Raum der textuellen Analyse zum Raum der Interpretation

Die ersten Analysen nach der Verfertigung des Einstellungsprotokolls bestehen noch vor allem aus Aufzählungen. Aufzählungen zum Material der Geschichte: Orte, Figuren, Ereignisse (erstes Kapitel: „Zwei Geschichten, keine Geschichte: der narrative Stoff des Films“) und Aufzählungen zu akustisch codierten Modalitäten (drittes Kapitel: „Ein Tonfilm, ein Musical, ein Sprechfilm [Untersuchung der Sub-Codes der Tonspur]") oder systematische Aufzählungen der Codes zur Raumkonstruktion (viertes Kapitel: „Dargestellter Raum/Konstruierter Raum“). Der Unterschied zum Einstellungsprotokoll besteht darin, dass man Figuren, Prozesse und Codes und keine isolierten Parameter aufzählt. Der Analytiker sucht Antworten auf Fragen des Typs: Wonach richtet sich der Aufbau der Geschichte? Wie wird der Raum geschaffen? Wie funktioniert die Tonspur? Der Analytiker ist nicht nur Beobachter, sondern eine Art Mechaniker: Er „zerlegt“ textuelle Mechanismen. Metz hat dieses etwas „sadistische“ Vergnügen an dieser Haltung gut erklärt: „Es gibt keine Sublimierung, wie Freud betont, ohne ,Entwirrung der Triebe““: „Das ist der theoretische Bruch, und wie alle Brüche bedeutet er ebenfalls eine Verbindung“, „eine spezifische Form von Wiedergutmachung“, die sich aus dem Verlust des Liebesgegenstands speist (Metz 2000 [1975/1977], 71f): ohne Frage eine sehr spezielle Erfahrung ...

Die Arbeit des „Textmechanikers“ entfaltet sich vollständig, wenn er koordiniert und Relationen herstellt, um zu verstehen, wie sich die Bedeutungs- und Affektproduktionen vollziehen. Der Analyst fokussiert zunächst Filmfragmente. Das fünfte Kapitel („Zwei kurze Montagen“) versucht, jenes Unwohlsein wiederzugeben, das den Zuschauer ereilt, wenn er die beiden Passagen sieht, und zeigt, dass dies aus einem „wahrnehmenden Verhalten resultiert“ (Bailblé/Marie/ Ropars 1974, 193), vorgegeben durch die filmische Bearbeitung, eine Bearbeitung, die „die Lektüre instabil macht, die Gegenwart schmerzhaft, die Sichtung unsicher“ (ibid., 192): schlechte Anschlüsse, Kostümwechsel, versetzte Blick- und Richtungsanschlüsse, zeitliche Brüche etc. Das sechste Kapitel („Über die Sequenzanalyse: Die Arbeit 
der écriture “), ${ }^{4}$ das sich den Einstellungen 435 bis 471 widmet, bestätigt diese Analyse, indem es die Produktion „einer fortschreitenden, schleichenden Unterwanderung des Realen“ zerlegt, die „einer Recherche nach einer immer direkteren Eroberung des Realen entspricht“ (ibid., 227). Die écriture von MURIEL in dieser Sequenz beruht also auf „einer Umformung des diegetischen Systems, das, gerade weil es umgeformt wird, als nicht ganz zerstört erscheint“, und, noch allgemeiner, wie eine Umformung der klassischen narrativen Sprache, die den „Unterschied“ würdigt und „an der Grenze zur Sinnhaftigkeit [...] in einer ständig wiederkehrenden Debatte um Sinn und Bedeutung“ arbeitet (ibid., 247). An diesem Punkt angekommen steht man einer Reihe von Texten gegenüber, die Analysen mit eingeschränkten Anwendungspunkten vorschlagen (bezogen auf eine Ebene oder ein Fragment), aber auch Analysen, die Tests entwerfen oder besser gesagt Sondierungen, die den ganzen Film betreffende Lektürehypothesen anbieten.

Das siebte Kapitel, „MurIEL, oder die Zeit einer Erzählung“, gibt eine „globale“ (ibid., 267) Lektüre des Films wider. Die Analyse des zeitlichen Systems der Erzählung erscheint in der Tat wie eine Achse, die erlaubt, die bis dahin erworbenen, unterschiedlichsten Vorgehen anzuwenden, um das narrative Gesamtbild des Films zu erfassen. Dies bringt Marie-Claire Ropars (die diese Analyse übernommen hat) dazu, einen Vorschlag zu unterbreiten, der nicht nur die Modalitäten der Sinnproduktion betrifft, sondern den Sinn des Films selbst: „Als Lektüre des Films schlage ich die Geschichte einer Vergangenheitsbewältigung vor, geführt aus einer doppelten Perspektive, die sich in der Geschichte von Alphonse und der von Bernard ausdrückt“ (ibid., 295). Das „Ich“ ist hier nicht unschuldig: Sobald der Forscher versucht, den gesamten Sinn eines Textes zu erfassen, handelt er nicht mehr als „Mechaniker“ des Textes, sondern als Subjekt, ein Subjekt, das seinen Text produziert, mit dem (hier zugegebenen) Risiko, den Raum der Analyse zu verlassen, um den Raum der Interpretation zu betreten.

4 [Anm. d. Ü.:] Für das Wort écriture, das im französischen Diskurs ein ,Schreiben' (oder eine ,Schreibweise' oder eine ,Handschrift') in verschiedenen Medien bezeichnet, gibt es im Deutschen keinen Ausdruck, der nicht irreführend wäre. Wir haben uns daher entschieden, das französische Wort beizubehalten. 
Der Raum der ideologischen Analyse

Bis hierhin blieben die durchgeführten Analysen innerhalb des Films. Die Lektüre von MuRIEL beabsichtigt aber, über dieses Vorgehen hinauszugehen: „Einmal entschlüsselt, muss dessen Bedeutung bewertet werden: Zur Diskussion steht weniger seine Natur als seine Funktion“ (ibid., 299). Die vorgeschlagenen Texte im achten Kapitel ergänzen dieses Anliegen und ordnen sich auf einer Achse der Relevanz ein, die dem Raum der ideologischen Analyse angehört (der Titel des Kapitels lautet „Bedeutung und Ideologie: der Text von MURIEL"): Diskussion der ideologischen Funktion der filmischen Form von MuRIEL; der Art, wie der Film über den Algerienkrieg spricht; der politischen Rolle des Films in Bezug auf das dominierende und auf das politisch-aktivistische Kino.

Der Operator der Analyse ist hier die (Re-)Konstruktion der drei historisch verankerten Kommunikationsräume durch die Forscher (der Film ist 1963 herausgekommen); natürlich sprechen die Forscher nicht von „Kommunikationsräumen“, sondern von „Kontext“, und dennoch handelt es sich genau darum:

- der Versuch einer Beschreibung der Zuschauererfahrung der 1960er Jahre während der Sichtung von MuRIEL, entweder ausgehend von der Analyse der Filmarbeit selbst (Ropars: „Texte“), oder von der Analyse der Publikumsreaktionen laut Presseberichten (Marie: „Kontext“): Konstruktion des Raums des Zuschauers;

- Analyse der „kritischen Lektüre von 1963“ („Kontext“; ibid, 327): Konstruktion des Raums der Kritik;

- Analyse der gesamten filmischen Gepflogenheiten des „Apparats der bürgerlichen Kultur“ (Bailblé: „Vor-Text“ und „Außer-Text“; ibid, 299): Konstruktion des Raums des Kinos in den 1960er Jahren.

Die Analysen lassen Divergenzen bei der Beurteilung des Intervenierens von MURIEL in diese verschiedenen Räume erkennen. Ropars ist der Ansicht, dass der Film den Zuschauer durch seine 
textuelle Arbeit „zu einer produktiven Aktivität“ führt: „Die Entschlüsselung einer sich ständig ändernden écriture macht aus dem Signifikat eine Frage, dessen zugleich vorgeschlagene und versteckte Beantwortung das Spektakel in Richtung des Zuschauers abdrängt und dabei den Ort seiner Bezugnahme offenlässt“ (ibid., 322). Marie, obgleich er mit der Analyse einverstanden ist (er sieht in MurIEL einen Brechtianischen Film), zeigt, dass in diesem Schreiben in Wirklichkeit einer der Gründe seines Scheiterns beim Publikum und bei der Kritik liegt. Bailblé ist schließlich der Kritischste der drei: Für ihn ist MURIEL ein „progressistischer Film für Progressisten“, kein revolutionärer Film und er bedauert, dass „Cayrol-Resnais 1963 ein Machtverhältnis zugutekam, das ihnen darüber hinaus ermöglichte, dem Positivismus des Volkes und den immer wieder aufkommenden ideologischen Veränderungen einen größeren Platz einzuräumen, selbst wenn sie dominiert werden“ (ibid., 348f). Wie soll man von seinen eigenen Überzeugungen abstrahieren, wenn man sich in derartige Analysen begibt? Die Erfahrung der Forschungsarbeit wird vom Engagement des Wissenschaftlers in der Gesellschaft durchkreuzt, von seiner staatsbürgerlichen Erfahrung. Zum Analytiker gesellt sich hier der Bürger [citoyen].

Der Raum der epistemologischen Reflexion

Verglichen mit der Filmlektüre durch einen Zuschauer situieren sich die eben präsentierten Analysen auf einer Meta-Ebene: Es geht darum, die Funktion des filmischen Textes zu hinterfragen und nicht nur zu produzieren; der analytische Text ist ein Text über einen Text. Aber in der Erfahrung der Lektüre von MuRIEL drückt sich von Anfang der Wille aus, darüber hinaus zu gehen; sie will reflexiv sein: „eine Methode erproben“ (ibid., 7). Die analytische Erfahrung wird gespalten und findet zu sich selbst zurück: die Erfahrung einer Erfahrung. Wir betreten den Raum der epistemologischen Reflexion, einen Raum auf der Meta-Meta-Ebene: Reflexion über die Analyse oder vielmehr Reflexion über den Operator (die Methode), der diese Analyse hervorbringt: das Problem der Beziehung zwischen allgemeinen Codes und „filmeigenen Codes“ (Analysen allgemeiner Codes „führen nicht 
zur Entdeckung eigener Codes“; ibid., 289); die Reflexion über den Ausdruck „Text“, über den Ausdruck „filmische écriture“ (227), über die Beziehungen zwischen Sinn und Bedeutung (,die Bedeutung geht aus einer Kombinatorik hervor, die über den Sinn entscheidet, keines der Materialien, die in dieser Kombinatorik zum Tragen kommen, kann nach einem eigenen, feststehenden System analysiert werden“; ibid, 239); Anerkennung der Tatsache, dass sich die textuellen Analyse nicht auf die semiologische Analyse reduzieren lässt: „Die Bedeutung entsteht im Aussetzen der Zeichen“ (241). Die Analyse stellt somit die Filmtheorie in Frage. Der analytische Text wird so auch zu einem theoretischen Text. Aus dem Analytiker wird schließlich ein Theoretiker.

Der Platz des Films im institutionellen universitären Raum

Letztlich zielt die Erfahrung der Lektüre von MuRIEL darauf ab, institutionelle Effekte im universitären Raum zu produzieren. Für die Wissenschaftler geht es darum, den Film als Forschungsobjekt an der Universität zu etablieren und ihn darüber hinaus als untersuchungswürdiges Objekt anzusehen, das heißt ihn von anderen Wissenschaftlern anerkannt zu wissen, von anderen Disziplinen und schließlich von der Institution. Denn auch wenn der Film zu dieser Zeit bereits an einigen Universitäten präsent war, so brauchte es noch einiges, um ihn wahrhaft anerkannt zu wissen (er sei „die Orchidee“ der Universität, hieß es seinerzeit). Der Operator dieser Anerkennungsunternehmung ist hier das Buch: Muriel, histoire d'une recherche. Es fällt schwer, das Novum darin heute einzuschätzen: Damals (wir befinden uns im Jahr 1974) gab es tausende von Büchern, die sich mit der Analyse literarischer Werke befassten, aber einem Film einen Text von 400 Seiten zu widmen, kam einer kleinen Revolution gleich. Es brauchte noch einige Jahre, bis der Film institutionell anerkannt wurde (Film als universitärer Studiengang etablierte sich erst in den 1990er Jahren), aber es ist nicht ausgeschlossen, dass diese Analyse von MURIEL einen gewissen Einfluss auf die Einsicht der Institution hatte. Die Forscher, die an dieser Erfahrung teilgenommen haben, 
nahmen somit die Rolle von Akteuren im Prozess der institutionellen Anerkennung des Films ein.

Was aus diesen drei Analysen festzuhalten bleibt, ist die Vielzahl von Kommunikationsräumen und konstruierten rezipierenden Aktanten, die Vielzahl an Operatoren und eingesetzten Modi der Bedeutungsproduktion, die Vielzahl produzierter Effekte (relationale Effekte, Erinnerungseffekte, „diskursive“ Effekte, ideologische Effekte, theoretische Effekte, institutionelle Effekte etc.) und die Vielzahl an hervorgebrachten Texten während jeder einzelnen dieser Lektüren. Die semiopragmatische Analyse widerlegt die immanentistische Illusion, es gäbe einen einzigen Text und eine einzige Lektüre. Im Vergleich zur immanentistischen Analyse haben wir es zugleich mit einer Pluralisierung und mit einer Heterogenisierung zu tun. Jede Lektüreerfahrung ist ein Palimpsest von Lektüren.

Die semiopragmatische Analyse zeigt schließlich, dass sogar in Abwesenheit der Migration von Produktionen in unterschiedliche Kontexte (wie es im vorigen Kapitel der Fall war), jede Lektüreerfahrung ihren Leser veranlasst, von Kommunikationsräumen zu Kommunikationsräumen zu wechseln, Räumen, die vom Leser einen Wechsel des Modus der Bedeutungsproduktion und der Affekte und darüber hinaus einen ganzen Rollenwechsel verlangen. Jede Lektüreerfahrung ist ein Abenteuer. 


\section{Schluss}

Die Pragmatik ist Gegenstand zahlreicher Kritik geworden. In Die unpersönliche Enunziation spricht Christian Metz ironisch von denen, die „mit großem Lärm bei Saussure und dem ,Strukturalismus‘ den Immanentismus und die Vernachlässigung des Sozialen [kritisieren], [...] sich aber wohlweislich davor [hüten], vor Ort nachzusehen“ (Metz 1997 [1991], 174). Ebenso Pierre Sorlin:

Weil sie nicht deutlich definiert, von welchem Publikum sie spricht, riskiert die Filmtheorie, einem abstrakten Pragmatismus zu huldigen, für den der Zuschauer eigentlich der Analysierende selbst ist, der anhand seiner eigenen Erfahrung sowie der seiner Umgebung untersucht, welche Effekte eines Films sich ihm eröffnen und welche sich ihm verschließen. $(1984,14 f)^{1}$

Und für Eliséo Véron „neigt die Pragmatik einerseits dazu, immer komplexere Kommunikationssituationen zu imaginieren (die aber deswegen nicht weniger arbiträr sind), und andererseits ein Ensemble von Regeln und sozialen Prinzipien zu postulieren, deren Status, Herkunft und kulturelle Gültigkeit unklar bleiben“ (1983, 101). Jüngeren Datums ist Bernard Mièges Liste jener Grenzen, an die laut ihm die „allgemeinen Theorien“ der Kommunikation stoßen, zu denen er auch „die meisten“ der pragmatischen Ansätze zählt: Reduktionismus, Abstraktion, Primat eines einheitlichen Paradigmas, Konfusion bei den betrachteten Instanzen, Abdriften ins Futurologische, Mangel an Prozeduren zur empirischen Überprüfung (1995, 83ff).

Ich werde nicht direkt auf diese Kritikpunkte eingehen, von denen manche das semiopragmatische Modell offensichtlich auch gar nicht betreffen (das Abdriften ins Futurologische, beispielsweise), aber sie bieten einen extrem nützlichen Reflexionsrahmen, um die theoretische Positionierung des vorgeschlagenen Modells besser zu verstehen.

1 [Anm. d. Ü.:] Zit. n. Kessler, Frank (2002): Historische Pragmatik. In: Montage AV 11,2, S. 104-112 (104). 
Es stimmt, dass das semiopragmatische Modell Anlass zum Vorwurf der Abstraktion bietet: Sein Top-down-Ansatz führt dazu, dass die Parameter, die es berücksichtig, a priori festgelegt werden. Aber ich denke, dass man zu dieser Abstraktion bedenkenlos stehen kann: Die Semiopragmatik hat nicht die gleichen Ziele wie die historische, soziologische oder ethnomethodologische Analyse. Die Art der Gültigkeit der semiopragmatischen Vorschläge kann bis zu einem gewissen Punkt mit der verglichen werden, die laut Metz den psychoanalytischen Ansatz und den der filmischen Enunziation kennzeichnet:

Hier wie dort geht man davon aus, im Besitz des notwendigen Instrumentariums an Kenntnissen und Methoden zu sein, wobei der gesamte Wert der Arbeit von persönlichen Fähigkeiten des Analytikers abhängt, denn er ist Forscher und zugleich mit dem Film auch Gegenstand der Forschung. Er kann behaupten, daß das spezifische Vergnügen am Spielfilm in einem fetischistischen Prozeß der Bewußtseinsspaltung, in einer Mischung von Glauben und Unglauben gründet. Er braucht dafür keine Probanden zu befragen, die eine derartige Frage ohnehin kaum beantworten könnten. Es handelt sich hier um eine generelle, oder präziser um eine generische Wahrheit. Sie betrifft den Zuschauer. Jeder kann sie in sich selbst finden und sie sagt uns - zum Beispiel - nicht, ob der Glaube bei diesen oder jenen unmittelbar zum Unglauben führt und ob im Gegensatz dazu bei anderen der Unglaube dominiert. Darin liegt nicht der geringste Widerspruch. Die generische Feststellung behält, wie ich denke, ihre übergeordnete Relevanz gegenüber ihren Varianten oder örtlichen Modalitäten. (Metz 1997 [1991], 26)

Der semiopragmatische Ansatz zielt darauf ab, generische Instrumente zu konstruieren, von den großen Regimen der Sinn- und Affektproduktion, die jeder in sich finden kann, den Modi, bis zu den Kommunikationsräumen. Als Theoretiker (re)konstruiere ich die Kommunikationsräume ausgehend von meiner Erfahrung der Wirklichkeit; ihre Bedingungssysteme sind in mir, so wie René Loureau $(1970,48)$ unser Ich als „Sammelsurium von Institutionen“ beschreibt. Man könnte 
auch sagen, dass die Instrumente der Semiopragmatik phänomenologisch erarbeitet sind.

Dennoch beansprucht das semiopragmatische Modell für die vorgeschlagenen Instrumente keine psychologische oder kognitive Existenz. Auch wenn sie einen phänomenologischen Ausgangspunkt haben (irgendwo muss man schließlich beginnen), bekommen sie innerhalb der Theorie einen rein heuristischen Status. Ihre Bewertung hängt davon ab, in welchem Maße sie dazu beitragen, die kommunikativen Prozesse aufzuklären.

Zwei Kriterien scheinen mir notwendig, um diesen Beitrag zu ermessen:

a) Ein heuristisches Modell ist desto wirksamer, je mehr Fragen es zu stellen erlaubt. Wie zu sehen war, hat dieses Anliegen von vornherein die Wahl der Form des Modells geleitet: ein Modell der NichtKommunikation, das impliziert, die Bedingungen in Frage zu stellen, die eine Kommunikation ermöglichen (siehe Einleitung).

b) Ein heuristisches Modell ist desto wirksamer, je mehr Differenzierungen es erlaubt: Die Semiopragmatik ermöglicht es, die Unterschiede zwischen verschiedenen Arten der Bedingung (Kapitel 1), verschiedenen Modi der Sinnproduktion (Kapitel 2 und 3) sowie verschiedenen Kommunikationskontexten und Lektüren zu erkennen, die diese Kontexte auslösen (Kapitel 4, 5 und 6).

Dieses Anliegen, Fragen und Differenzierungen $\mathrm{zu}$ formulieren, erklärt, wieso das semiopragmatische Modell so rigide im Umgang mit den möglichst restriktiv definierten Begriffen ist. Auch hier denke ich, dass es richtig ist, diese reduktive Position einzunehmen.

Ein heuristisches Modell ist im Wesentlichen ein Korpus an Definitionen, eine „methodologische[] Sprache [...], die die deskriptiven Begriffe definiert“, wie Greimas (1971 [1966], 11) es ausdrückt. Der Ausdruck „Begriff“ ist zwar zu stark und ich bevorzuge, wie gesagt, den Ausdruck „Werkzeug“, aber die Formulierung von Greimas fasst meine Konzeption des semiopragmatischen Modells gut zusammen. Diese Definitionen führen im Allgemeinen zu einer Reihe von Fragen, welche sie entfalten und präzisieren: Welche Form soll man dem Modell geben? Wie sind die Modi zu konstruieren? Wie funktioniert 
dieser oder jener Modus? Wie ist ein Kommunikationsraum zu analysieren? ...

Am Ende dieses Parcours hat der Leser also eine Reihe von Instrumenten an der Hand (siehe die Liste auf der folgenden Seite), die ihm hoffentlich helfen können, in einem gegebenen Kontext das Funktionieren der Kommunikation zu befragen. Und wie die letzten drei Kapitel gezeigt haben, hat dieses abstrakte Modell durchaus die Ambition, die Kommunikation zu untersuchen, wie sie in der Wirklichkeit funktioniert. 


\section{Liste der vorgeschlagenen Instrumente}

immanentistisches Paradigma, S. 33ff.

pragmatisches Paradigma, S. 33ff.

Semiopragmatik, S. 39ff.

Modell, S. 41ff.

Aktanten, S. 44ff.

Kontext, S. 47ff.

natürliche Bedingungen, S. 50ff.

narrative Bedingungen, S. 53ff.

sprachliche Bedingungen, S. 57ff.

nicht-natürliche Bedingungen, S. 61ff.

kommunikative Kompetenz, S. 69ff.

diskursive Kompetenz, S. 69f.

semio-linguistische Kompetenz, S. 70

soziokulturelle Kompetenz, S. 70

referenzielle Kompetenz, S. 70

Kommunikationsraum, S. 70ff.

Diskursiver Raum, S. 70

Westlicher Raum, S. 71

Modus, S. 71f.

vertikaler vs. horizontaler Modus, S. 91

fiktionalisierender Modus (erste Annäherung), S. 76

fiktionalisierender Modus (neuer Vorschlag), S. 87

spektakularisiender Modus, S. 78

energetischer Modus, S. 79

dokumentarisierender Modus, S. 82

moralisierender Modus, S. 82

fabularisierender Modus, S. 88

ästhetischer Modus, S. 96

künstlerischer Modus (reduzierte Form), S. 100

künstlerischer Modus (vollständige Form), S. 105

intimer Modus, S. 111 
privater Modus, S. 118

Modus der Zeugenschaft, S. 127

Modus der Authentizität, S. 139

„diskursiver“ Modus, S. 143

Operatoren, S. 118f.

Beziehungen, Effekte, S. 130f.

(Analyse des Prozesses der) Migration, S. 133ff.

Textuelle Analyse (im semiopragmatischen Ansatz), S. 155

\section{Etappen der Konstruktion des semiopragmatischen „Modells“}

Schema 1, S. 43

Schema 2, S. 48

Schema 3, S. 67

Schema 4, S. 90

Schema 5, S. 132 


\section{Literatur}

Adrien, Jean-Louis / Gattegno, Maria Pilar (2004): Dépistage précoce de l'autisme à l'aide des films familiaux: apport de la recherche et d'une démarche rétrospective dans la démarche de soins. In: Alain Haddad, Antoine Guedeney \& Tim Greacen (Hg.), Santé mentale du jeune enfant: prévenir et intervenir. Toulouse: Erès. S. 85-93.

Allard, Laurence (1995): Une rencontre entre film de famille et film expérimental. In: Odin 1995, S. 113-126.

Arnaud, Alain / Chandelier, Michel (2000): La prise en charge du Tour de France par la télévision, dans le contexte de la crise du dopage. 1998-99. In: Pierre Gabaston \& Bernard Leconte (Hg.), Sports et télévision. Regards croisés. Paris: L’Harmattan, S. 247265.

Arnauld, Antoine / Lancelot, Claude (1999): Grammaire générale et raisonnée [1660]. Genf: Slatkine.

Austin, John L. (2002): Zur Theorie der Sprechakte [engl. 1962]. Aus dem amerikanischen Englisch von Eike von Savigny. Stuttgart: Reclam.

Bailblé, Claude / Marie, Michelle / Ropars, Marie-Claire (1974): Muriel, histoire d'une recherche. Paris: Éditions Galilée.

Barthes, Roland (1974): Die Lust am Text [frz. 1973]. Frankfurt a.M.: Suhrkamp.

— (2010): Mythen des Alltags. Vollständige Ausgabe [frz. 1957]. Aus dem Französischen von Horst Brühmann. Frankfurt a. M.: Suhrkamp.

Baudry, Jean-Louis (2003): Das Dispositiv. Metapsychologische Betrachtungen des Realitätseindrucks [frz. 1975]. Aus dem Französischen von Max Looser. In: Robert F. Riesinger (Hg.), Der kinematographische Apparat. Münster: Nodus, S. 41-62.

Bellour, Raymond (2001): The Analysis of Film [frz. 1979]. Aus dem Französischen von Bertrand Augst, Ben Brewster, Nancy Huston, Roxanne Lapidus, Diana Matias, Mary Quaintance, Inge Pruks, Hillary Radner, Maureen Turim; hg. v. Constance Penley. Bloomington / Indianapolis: Indiana University Press.

- (2009): Le corps au cinéma. Hypnoses, emotions, animalités. Paris: P.O.L.

Berrendonner, Alain (1981): Elements de pragmatique linguistique. Paris: Minuit.

Bolter, Jay David / Grusin, Richard (2000): Remediation. Understanding New Media. Cambridge, MA: MIT Press.

Bourdieu, Pierre (1982): Die feinen Unterschiede. Kritik der gesellschaftlichen Urteilskraft [frz. 1979]. Aus dem Französischen von Bernd Schwibs \& Achim Russer. Frankfurt a. M.: Suhrkamp.

Brémond, Claude (1973): Logique du récit. Paris: Seuil.

Brown, Patricia Fortini (1998): Renaissance in Venedig. Kunst und Kultur in der Stadt Dogen [engl. 1997]. Köln: DuMont.

Caillois, Roger (2004): Die Schrift der Steine [frz. 1970]. Graz: Droschl.

Canudo, Ricciotto (2016): Die Geburt einer sechsten Kunst. Versuch über den Kinematographen [ital. 1908/frz. 1911]. In: Margrit Tröhler \& Jörg Schweinitz (Hg.), Die Zeit des Bildes ist angebrochen. Französische Intellektuelle, Künstler und Filmkritiker über das Kino. Eine historische Anthologie 1906-1929. Berlin: Alexander, S. 71-86.

Casetti, Francesco / Odin, Roger (2001): Vom Paläo- zum Neo-Fernsehen. Ein semiopragmatischer Ansatz [frz. 1990]. Aus dem Französischen von Frederike Eggs. In: 


\section{Literatur}

Casetti, Francesco / Odin, Roger (2001): Vom Paläo- zum Neo-Fernsehen. Ein semiopragmatischer Ansatz [frz. 1990]. Aus dem Französischen von Frederike Eggs. In: Ralf Adelmann, Jan O. Hesse, Judith Keilbach, Markus Stauff \& Matthias Thiele (Hg.), Grundlagentexte zur Fernsehwissenschaft. Theorie - Geschichte - Analyse. Konstanz: UVK, S. 311-333.

Casey, Edward S. (2000): Remembering: A Phenomenological Study [1987]. 2. Aufl. Bloomington: Indiana University Press.

Castoriadis, Cornelius (1984): Gesellschaft als imaginäre Institution. Entwurf einer politischen Philosophie [frz. 1975]. Aus dem Französischen von Horst Brühmann. Frankfurt a.M.: Suhrkamp.

Chalfen, Richard (1987): Snapshot. Versions of Life. Bowling Green: Bowling Green State University Press.

Chomsky, Noam (1971): Cartesianische Linguistik. Ein Kapitel in der Geschichte des Rationalismus [engl. 1966]. Aus dem amerikanischen Englisch von Richard Kruse. Tübingen: Niemeyer.

— (1973): Strukturen der Syntax [engl. 1965]. Aus dem amerikanischen Englisch von KlausPeter Lange. Den Haag \& Paris: Mouton / Berlin: DeGruyter.

Clemens, Éric (1993): La fiction et l'apparaître. Paris: Albin Michel.

Colin, Michel (1985): Langue, film, discours. Prolégomènes à une sémiologie generative du film, Paris: Klincksieck.

— (1990): Comprendre l'événement sportif à la télévision. L’exemple de la course cycliste. In: Communications 51, S. 79-110.

Cowen, Paul S. (2002): L'importance des processus cognitifs et de la recherche empirique en études cinématographiques. In: CiNéMAS12,2, S. 39-60.

Dayan, Daniel (1983): Western graffiti: Jeux d'images et programmation du spectateur dans La chevauchée fantastique de John Ford. Paris: Clancier-Guenaud.

— / Katz, Elihu (1992): Media Events: The Live Broadcasting of History. Cambridge: Harvard University Press.

Deacon, Terrence (1997): The Symbolic Species. New York: W.W. Norton \& Company.

Degli, Martine / Mauzé, Marie (2000): Arts premiers. Le temps de la reconnaissance. Paris: Découvertes Gallimard.

Dickie, George (1988): Evaluating Art. Philadelphia, PA: Temple University Press.

Eco, Umberto (1972): Einführung in die Semiotik [ital. 1968]. Aus dem Italienischen von Jürgen Trabant. München: Fink.

— (1973): Das offene Kunstwerk [ital. 1962]. Aus dem Italienischen von Günter Memmert. Frankfurt a.M.: Suhrkamp.

— (1992): Die Grenzen der Interpretation [ital. 1990]. Aus dem Italienischen von Günter Memmert. München: Hanser.

— (2009): Die unendliche Liste [ital. 2009]. München: Hanser.

Fauconnier, Gilles (1984): Espaces mentaux. Aspects de la construction du sens dans les langues naturelles. Paris: Minuit.

Fish, Stanley (1980): Is There a Text in This Class? The Authority of Interpretative Communities. Harvard: Harvard University Press.

Flichy, Patrice (2010): Le sacre de l'amateur. Sociologie des passions ordinaires à l'ère du numérique. Paris: Seuil.

Fodor, Jerry (1975): The Language of Thought. Cambridge: Harvard UP. 


\section{Literatur}

Gaboriau, Philippe (1995): Le Tour de France et le vélo. Histoire sociale d'une épopée contemporaine. Paris: L'Harmattan.

Gaudreault, André / Odin, Roger (2001): Le Cinématographe, un „enfant prodige“ ou l'enfance de la cinématographie. In: Leonardo Quaresima \& Laura Vicchi (Hg.), $\mathrm{La}$ decima muta. Le cinema e le altre arte. Udine / Gemona de Friuli: Forum, S. 67-81.

Genette, Gérard (1994): L'œuvre d'art. Paris: Seuil.

- (1997): La relation esthétique. Paris: Seuil.

Gervereau, Laurent (2000): Les images mentent. Histoire du visuel au XXe siècle. Paris: Seuil.

Goffman, Erwing (1977): Rahmen-Analyse. Ein Versuch über die Organisation von Alltagserfahrungen [engl. 1974]. Aus dem amerikanischen Englisch von Hermann Vetter. Frankfurt a.M.: Suhrkamp.

Goodman, Nelson (1997): Sprachen der Kunst. Entwurf einer Symboltheorie [engl. 1976]. Aus dem amerikanischen Englisch von Bernd Philippi. Frankfurt a. M.: Suhrkamp.

Goody, Jack (1977): The Domestication of the Savage Mind. Cambridge: Cambridge University Press.

- (1997): Representations and Contradictions: Ambivalence Towards Images, Theatre, Fiction, Relics and Sexuality. Oxford / Malden, MA: Blackwell.

Gordon, Peter (2004): Numerical Cognition Without Words. Evidence from Amazonia. In: Science Magazine 306, 5695, S. 496-499.

Greimas, Algirdas Julien (1971): Strukturale Semantik. Methodologische Untersuchungen [frz. 1966]. Aus dem Französischen von Jens Ihwe. Braunschweig: Vieweg + Sohn.

Gribenski, Michel (2005): Musique et sciences cognitives. In: Labyrinthe 20, S. 99-116.

Grodal, Torben (1997): Moving Pictures. A New Theory of Film Genres, Feelings, and Cognition. Oxford: Clarendon Press.

Guibert, Hervé (1981): L’image fantôme. Paris: Éditions de Minuit.

Halbwachs, Maurice (1985): Das Gedächtnis und seine sozialen Bedingungen [frz. 1925]. Übers. von Lutz Geldsetzer. Frankfurt a. M.: Suhrkamp.

Hamburger, Käte (1987): Die Logik der Dichtung [1957]. 3. Auflage. München: Klett-Cotta \& dtv.

Hymes, Dell (1974): Foundations in Sociolinguistics. Philadelphia: University of Pennsylvania Press.

Jakobson, Roman (1971): Linguistik und Poetik [engl. 1960]. In: Jens Ihwe (Hg.): Literaturwissenschaft und Linguistik. Ergebnisse und Perspektiven, Frankfurt a.M.: Athenäum, S. 142-178.

Jenkins, Henry (2006): Convergence Culture. Where Old and New Media Collide. New York: New York University Press.

Journot, Marie-Thérèse / Duchet, Chantal (1995): Du privé au publicitaire. In: Odin 1995, S. 177-190.

Jullier, Laurent (1997): L'écran post-moderne. Un cinéma de l'allusion et du feu d'artifice. Paris: L'Harmattan.

- (2002): Cinéma et cognition. Paris: L’Harmattan.

Kerbrat-Orrechioni, Catherine (1980): L'énonciation. De la subjectivité dans le langage. Paris: A.Colin.

— (1986): L'implicte. Paris: A.Colin.

Lafond, Jean-Daniel (1982): Le film sous influence. Un procédé d'analyse. Paris: Édilig.

Latraverse, Francois (1987): La pragmatique. Histoire et critique. Brüssel: Mardaga. 


\section{Literatur}

Leners, Jean-Claude (2009): Reminiscene: A Way to Use Amateur Films in Order to Work with Patients suffering from Memory Problems. In: Sonja Kmec \& Viviane Thill (Hg.), Private Eyes and the Public Gaze: The Manipulation and Valorisation of Amateur Images. Trier: Kliomedia, S. 97-99.

Lerdahl, Fred / Jackendoff, Ray (1983): A Generative Theory of Tonal Music. Cambridge: MIT Press.

Lieberman, Philip (1998): Eve Spoke, Human Language and Human Evolution. New York: W.W. Norton \& Company.

Loureau, René (1970): L'analyse institutionnelle. Paris: Minuit.

Maquet, Jacques (1986): The Aesthetic Experience. An Anthropologist Looks at the Visual Arts. New Haven, CT: Yale University Press.

Marin, Louis (1978): Le récit est un piége. Paris: Minuit.

Mary, Bertrand (1993): La Photo sur la cheminée. Naissance d'un culte moderne. Paris: Métailié.

Maugras, Émile / Guégan, Maurice (1908): Le cinématographe devant le droit. Paris: V. Giard \& E. Brière.

Mellier, Denis (2003): L'interrogation psyché-soma et le temps de l'intrigue, ce que nous apprennent les bébés. In: Champ Psychosomatique 30, S. 27-43.

Metz, Christian (1972): Semiologie des Films [frz. 1964-1968]. Aus dem Französischen von Renate Koch. München: Fink.

— (1973): Sprache und Film [frz. 1971]. Aus dem Französischen von Micheline Theune \& Arno Ros. Frankfurt a.M.: Athenäum.

- (1977): Essais sémiotiques. Paris: Klincksieck.

- (1997): Die unpersönliche Enunziation oder der Ort des Films [frz. 1991]. Aus dem Französischen von Frank Kessler, Sabine Lenk und Jürgen E. Müller. Münster: Nodus.

— (2000): Der imaginäre Signifikant. Psychoanalyse und Kino [frz. 1975/1977]. Aus dem Französischen von Dominique Blüher, Thomas Hübel, Elisabeth Madlener, Robert F. Riesinger, Peter Stolle, Margrit Tröhler und Michael Wiesmüller. Münster: Nodus.

Moine, Raphaëlle (2002): Les genres du cinema. Paris: Nathan.

Moran, James M. (2002): There's No Place Like Home Video. Minneapolis: University of Minnesota Press.

Nora, Pierre (1990): Zwischen Geschichte und Gedächtnis [frz. 1984]. Übers. von Wolfgang Kaiser. Berlin: Wagenbach.

Odin, Roger (Hg.) (1995): Le film de famille. Usage privé, usage public. Paris: Méridiens Klincksieck.

- (Hg.) (1999): Communication 68 („Le cinéma en amateur“).

- (2000): De la fiction. Brüssel: DeBoeck.

- (2002): Kunst und Ästhetik bei Film und Fernsehen. Elemente zu einem semiopragmatischen Ansatz. In: Montage AV 11, 2, S. 42-57.

Orians, Gordon H. / Heerwagen, Judith H. (1992): Evolved responses to landscapes. In: Jerome H. Barkow, Leda Cosmides \& John Tooby (Hg.), The Adapted Mind. Oxford: Oxford University Press, S. 555-579.

Parret, Herman (1993): The Aesthetics of Communication: Pragmatics and Beyond. Dordrecht: Springer.

Peirce, Charles S. (2000): Semiotische Schriften Bd. I [engl. 1865-1903]. Hg. u. übers. v. Christian J. W. Kloesel \& Helmut Pape. Frankfurt a. M.: Suhrkamp.

Peiry, Lucienne (1999): L’Art Brut. Die Träume der Unvernunft [frz. 1997]. Jena: Glaux. 


\section{Literatur}

Perec, Georges (1975): Approche de quoi. In: Le pourrissement des sociétés. Paris: 10/18, S. 251-255.

Pica, Pierre / Lemer, Cathy / Izard, Véronique / Dehaene, Stanislas (2004): Exact and Approximate Arithmetic in an Amazonian Indigene Group. In: Science Magazine 306, 5695, S. 499-503.

Pomian, Krysztof (1990): Musée et patrimoine. In: Henri-Pierre Jeudy (Hg.): Patrimoine en folie. Paris: Maison des Sciences de l'Homme. S. 177-198.

Price, Sally (1992): Primitive Kunst in zivilisierter Gesellschaft [engl. 1989]. Frankfurt a. M.: Campus.

Prudhomme, Claude (2010): Occident. In: Olivier Christin (Hg.), Dictionnaire des concepts nomades en sciences humaines. Paris: Métailié, S. 343-362.

Rancière, Jacques (2006): Die Aufteilung des Sinnlichen. Die Politik der Kunst und ihre Paradoxien [frz. 2000]. Aus dem Französischen von Maria Muhle, Susanne Leeb \& Jürgen Link. Berlin: b_books.

Revel, Jacques (Hg.) (1996): Jeux d'échelles. La microanalyse à l'expérience. Paris: EHESSGallimard-Seuil.

Ricœur, Paul (2004): Gedächtnis, Geschichte, Vergessen [frz. 2000]. Aus dem Französischen von Hans D. Gondek, Heinz Jatho \& Markus Sedlaczek. Paderborn: Fink.

Rochlitz, Rainer (1994): Subversion et subvention. Paris: Gallimard.

Saussure, Ferdinand de (2001): Grundfragen der allgemeinen Sprachwissenschaft [frz. 1916]. Aus dem Französischen von Herman Lommel. Berlin / New York: De Gruyter.

Schaeffer, Jean-Marie (1987): L'image précaire. Du dispositif photographique. Paris: Seuil.

- (1992): L'art de l'âge moderne. L'esthétique et la philosophie de l'art du XVIIIe au nos jours. Paris: Gallimard.

Schaff, Adam (1965): Langage et realité. In: Diogène 51, S. 153-175.

Searle, John (1971): Sprechakte. Ein sprachphilosophischer Essay [engl. 1969]. Aus dem amerikanischen Englisch von Rolf \& Renate Wiggershaus. Frankfurt a. M.: Suhrkamp.

Shannon, Claude E. / Weaver, Warren (1976): Mathematische Grundlagen der Informationstheorie [engl. 1949]. Aus dem Englischen von Helmut Dressler. München / Wien: Oldenbourg.

Shusterman, Richard (1994): Kunst Leben. Die Ästhetik des Pragmatismus [engl. 1992]. Aus dem amerikanischen Englisch von Barbara Reiter. Frankfurt a. M.: Fischer.

Sierek, Karl (1990): „Hier ist es schön.“ Sich sehen im Familienkino. In: Christa Blümlinger (Hg.), Sprung im Spiegel. Filmisches Wahrnehmen zwischen Fiktion und Wirklichkeit. Wien: Sonderzahl, S. 147-167.

Sorlin, Pierre (1984): Promenades dans Rome. In: Iris 2,2, S. 5-16.

- (1992): Esthétique de l'audiovisuel. Paris: Nathan.

Sperber, Dan / Wilson, Deirdre (1986): Relevance. Communication and Cognition. Oxford: Basil Blackwell.

Spielberg, Steven (2004): Kino ist meine Teufelsaustreibung, Interview mit Steven Spielberg. In: Die Zeit 36.

[http://video.zeit.de/2004/36/InterviewSpielberg/komplettansicht].

Staiger, Janet (2000): Perverse Spectators: The Practices of Film Reception. New York: New York University Press.

Stern, Daniel (1990): Engagements subjectifs. Le point de vue de l'enfant, In: André Carel, Jacques Hochmann \& Henri Vermorel (Hg.), Le Nourisson et sa famille. Lyon: Cesura Lyon Editions, S. 30-45. 


\section{Literatur}

- (1998): Die Mutterschaftskonstellation. Eine vergleichende Darstellung verschiedener Formen der Mutter-Kind-Psychotherapie [engl. 1995]. Stuttgart: Klett-Cotta.

Tisseron, Serge (2001): L'intimité surexposée. Paris: Hachette.

Todorov, Tzvetan / Ducrot, Oswald (1975): Enzyklopädisches Wörterbuch der Sprachwissenschaften [frz. 1972]. Aus dem Französischen von Micheline Theune-Baube und Arno Ros. Frankfurt a.M.: Akademische Verlagsgesellschaft Athenaion.

Trevoz, Michel (1995): L'art brut. Genf: Skira.

Trousset, Jules (1877): Nouveau Dictionnaire Encyclopédique (5 Bände). Paris: La Librairie Illustrée.

Véron, Eliséo (1983): Il est là, je le vois, il me parle. In: Communications 38, S. 98-120.

Watzlawick, Paul / Beavin, Janet H. / Jackson, Don D. (2011): Menschliche Kommunikation Formen, Störungen, Paradoxien [engl. 1967]. (12. unveränd. Aufl.). Bern: Huber.

Weitz, Morris (1956): The Role of Theory in Aesthetics. In: The Journal of Aesthetics and Art Criticism 15,1, S. 27-35.

Whorf, Benjamin Lee (1963): Sprache, Denken, Wirklichkeit. Beiträge zur Metalinguistik und Sprachphilosophie [engl. 1956]. Reinbek: Rowohlt.

Willett, Frank (2002): African Art (1971/1993). New York: Thames \& Hudson.

Winkin, Yves (2001): Anthropologie de la communication. De la théorie au terrain. Brüssel:

DeBoeck / Paris: Seuil.

- (Hg.) (1981): La nouvelle communication. Paris: Seuil.

Zinoviev, Alexandre (1995): L'occidentisme. Essai sur le triomphe d'une idéologie. Paris: Plon. 


\section{Nachwort von Roger Odin zur deutschen Ausgabe}

\section{Die Semiopragmatik heute}

Im vorliegenden Buch habe ich den allgemeinen Rahmen des semiopragmatischen Ansatzes dargestellt. Seit seinem Erscheinen 2011 hat sich dieser Rahmen nicht verändert, einzelne Punkte wurden jedoch - durch mich oder andere - weiterentwickelt oder ergänzt. Das betrifft insbesondere die Anwendung des Modells auf Gegenstände, die von jenen, die ursprünglich zu seiner Ausarbeitung führten, recht weit entfernt sind (wie etwa Videoclips, Webserien, Interfaces, interaktive Dokumentarfilme, Autobiografien, Tonmontage, Tanz, Malerei, Gesang, digitale Bücher oder Lehrbücher). Den „Gegenstand zu wenden“, wie Christian Metz es nannte (2017 [1975], 126), führt oft zu einer Weiterentwicklung des Modells.

Persönlich sehe ich die größte Weiterentwicklung darin, die Bedeutung mentaler Räume anzuerkennen (Odin 2016a; 2016b). Laut René Lourau ist „unser Ich ein Sammelsurium von Institutionen“ $(1970,48)$. Mir scheint, man könnte auch sagen, dass es ein Sammelsurium von Kommunikationsräumen ist, von denen manche Institutionen sind, andere nicht. Diese von uns internalisierten Räume nenne ich „mentale Räume“. Der Status dieser Räume entspricht entweder existierenden Räumen (Kino, Familie, Schule etc.), die wir verinnerlicht haben und die in vielen Kommunikationssituationen eine Rolle spielen (zum Beispiel kann uns ein Film, den wir im Fernsehen sehen, an den Kinosaal erinnern und uns die geringe Größe des Bildschirms, das Fehlen eines abgeschirmten Raums oder die schlechte Tonqualität bedauern lassen). Oder er hat kein Pendant in der Wirklichkeit und es handelt sich um begehrte oder erträumte Räume, die noch nicht physisch erschaffen wurden. So war das Kino vor seiner Transformation in einen existierenden Raum im Jahr 1895 ein erträumter Kommunikationsraum (zahlreiche Texte und Dispositive bezeugen 
seine Existenz). ${ }^{1}$ Dieser zweite Raumtyp ist übrigens der Ursprung der meisten Erfindungen.

In einem Vortrag auf der Konferenz „La magie des effets spéciaux“ („Der Zauber der Spezialeffekte“) ${ }^{2}$ habe ich dargelegt, wie bestimmte Effekte, die wir verwenden, wenn wir mit unseren Smartphones fotografieren oder filmen, in einem bestimmten Verhältnis zu mentalen Räumen stehen: dem der analogen Fotografie (Sepia-Effekt, Schwarzweiß usw.), dem des Stummfilms (Schwarzweiß, fehlender Ton, Flackern, beschleunigte Bewegung usw.) oder dem des Amateurfilms (Super8-Effekt: Streifen, Sprünge, Vorherrschen bestimmter Farben, Geräusch des Projektors etc.) (Odin 2019).

Zudem habe ich verschiedene Instrumente vorgeschlagen, um die Beziehung zwischen realen und mentalen Räumen zu erläutern. Als Introdukteur bezeichne ich alles, was dazu beiträgt, die Beziehung zwischen mentalem und physischem Raum zu verhandeln. ${ }^{3}$ Unterscheiden lassen sich diskursive Introdukteure (z.B. die Ab- und Vorspänne, die im Fernsehen vor Spielfilmen gezeigt werden und dazu beitragen, den Raum des Kinos in den des Fernsehens zu integrieren) und pragmatische Introdukteure: Dispositive, die dazu dienen, den physischen dem mentalen Raum anzunähern (z.B. das Heimkino). Als Konnekteur bezeichne ich Instrumente, die beschreiben sollen, wie wir die mentalen Räume in einem gegebenen realen Raum aufrufen. Von einem rigiden exklusiven Konnekteur spreche ich, wenn ich den realen und den mentalen Raum radikal trenne (in dieser Perspektive ist ein im Fernsehen gesehener Film durchaus kein Kino); dagegen spreche ich von einem rigiden inklusiven Konnekteur, wenn ich umgekehrt bemüht bin, die Spezifik der Erfahrung eines mentalen Raums in

1 [Anm. d.Ü.:] Odin denkt hier offenbar an utopische Romane wie L'Ève future (Auguste Villiers de l'Isle-Adam, 1886) und optische Apparate zur Bewegtbilderzeugung wie Phenakistiskop, Zoetrop oder Praxinoskop.

2 Die Konferenz wurde von GRAFICS unter der Leitung von André Gaudreault, Martin Lefebvre und Viva Paci organisiert und fand vom 5. bis 10. November 2013 in der Cinémathèque québécoise in Montréal statt.

3 [Anm. d. Ü.:] Für das französische Wort „introducteur“ wie auch für das nachfolgende „conncecteur“ gibt es leider keine brauchbaren deutschen Pendants. Die Möglichkeit von „Einführern“ und „Verbindern“ zu sprechen, haben wir verworfen, da sie zu sehr nach menschlichen Wesen klingen. Daher fiel die Wahl (auf die zugegebenermaßen auch nicht schönen) Neologismen „Introdukteur“ und „Konnekteur“. 
einem anderen realen Raum aufrechtzuerhalten (z.B. wenn ich in der U-Bahn auf dem Smartphone einen Film als Kinofilm gucke, indem ich um mich herum das erzeuge, was Francesco Casetti und Sara Sampietro [2012] eine „existentielle Blase“ nennen); von einem flexiblen Konnekteur, wenn ich mich den gegebenen Unterschieden zwischen den Räumen anpasse (eine Art Aushandlung); und schließlich von einem offenen Konnekteur, wenn ich an den Veränderungen des mentalen Raums durch die realen Räume Gefallen finde (z.B. einen Film auf verschiedenen Screens schauen: Kinoleinwand, Fernsehbildschirm, Smartphone, Tablet etc.).

Auch erschien es mir notwendig, einen neuen Modus $\mathrm{zu}$ konstruieren: den des Making of (Odin 2012, 84f). Während des ersten Pocket Film Festivals (2005) wurde mir klar, dass das Publikum in diesem Raum eine Erwartung folgender Art hegte: Was hat der Regisseur anders gemacht, weil er mit einem Smartphone statt mit einer Kamera filmte? Davon ausgehend sahen sich die Zuschauer herausgefordert, das Objekt Smartphone zu untersuchen: eine Analyse auf technischer Ebene (wie spielt der Filmemacher mit den Beschränkungen des Smartphones, vor allem mit der sehr geringen Bildauflösung? $\left.{ }^{4}\right)$; eine Analyse auf der Ebene der Form des Smartphones und seiner Beziehung zur Hand; eine funktionale Analyse (in welcher Beziehung steht der Film zu den vielen Funktionen des Smartphones? Wie bezieht der Film die Tatsache mit ein, dass das Smartphone ein soziales Objekt ist, das sich in einen bestimmten Typ von Situationen und Beziehungen einschreibt?). Dies ist eine völlig andere Positionierung als die des Kinozuschauers: Wer stellt sich schon Fragen über den Produktionsapparat, wenn er einen Kinofilm schaut? Daher der Vorschlag: Der Modus des Making of zeichnet sich durch eine Sinnproduktion aus, die auf Fragen hinsichtlich des Tuns fokussiert (wie wurde das gemacht?), woraus folgt, dass man sich weniger für den Inhalt der Kommunikation interessiert. Als ich 1999 für eine Ausgabe von Communications über den Raum des Amateurfilms schrieb (Odin 1999), habe ich bereits auf diese Art der Sinnproduktion

42005 hatten die Kameras der Telefone noch eine sehr geringe Bildauflösung, was sie für die Künstler visuell interessant werden ließ; heute ist die Auflösung „zu gut“, das Bild hat seine Materialität, seine optische Spezifik verloren. 
hingewiesen (ohne allerdings die Bezeichnung „Modus des Making of“ zu verwenden): In Amateurfilmclubs beschränken sich die Diskussionen oft auf Fragen des technischen Know-hows, das beim Dreh relevant ist (welches Filmmaterial? welches Objektiv? welche Art der Beleuchtung?) und der Inhalt wird fast vollständig ignoriert.

Es ist kaum erstaunlich, dass dieser Lektüremodus heute immer verbreiteter wird, denn mit dem Smartphone (eine Kamera, die wir die ganze Zeit in der Tasche dabei haben) sind wir alle zu Amateurfilmern geworden. Davon abgesehen tragen auch die vielen auf DVD veröffentlichten Making ofs des jeweiligen Films dazu bei, dass die Zuschauer diese Lektüre annehmen, wie Claudia Lanbach in ihrer Analyse der Rezeption des Kurzfilms DOT (Ed Patterson \& Will Studd, 2010) gezeigt hat:

Manche Berichte legen nahe, dass die Zuschauer wegen der technischen Glanzleistung dieser Arbeit auch das Making of angeschaut haben. Das hat dazu beigetragen, dass die technischen und technologischen Aspekte des Films auch von nichtprofessionellen $\mathrm{Zu}$ schauern beachtet wurden (Lanbach 2018, 287).

Für das Feld der Malerei gibt David Hockney in seinem Buch Secret Knowledge: Rediscovering the Lost Techniques of the Old Masters (2001) ein fast schon karikaturartiges Beispiel der Anwendung dieses Modus. Er zeigt, dass ab einem bestimmten Datum in der Geschichte der Malerei (dem Beginn des 15. Jahrhunderts) ein radikaler Wandel stattgefunden hat und dass dieses Datum dem Zeitpunkt entspricht, ab dem die Maler Zugang zu optischen Dispositiven hatten (Camera Obscura, Camera Lucida usw.), die es ihnen ermöglichten, eine genauere Abbildung der Wirklichkeit zu erzielen. Bemerkenswert ist an diesem Werk besonders die extreme Präzision des Autors in seinen Analysen entlang der Relevanzachse „Wie wurde das gemacht?“, ohne je ein Wort über den Inhalt zu verlieren. Auch das Feld der Literatur ist vor diesem Lektüremodus nicht gefeit: In seinem Roman Exit Ghost beschwert sich Philip Roth mittels seiner Protagonisten über die Tatsache, dass sich die Kritiker bei ihrer Lektüre oft für das „kleine biografische Detail“ oder für die „vermeintliche Quelle dieser oder 
jener Aussage“ interessieren und „das Buch in solchen Überlegungen ertränken“ (dies ist eine andere Modalität des Making of-Modus) (vgl. Savigneau 2009).

Andere Forscher haben andere Modi vorgeschlagen (den reflexiven, ludischen, taxonomischen, hermeneutischen, performativen, autobiografischen, interaktiven usw. Modus). Ich möchte allerdings auf die Gefahr hinweisen, Modi ad hoc für einen Produktionstyp zu konstruieren. In meiner Vorstellung gehören sie vielmehr zur kulturellen Kompetenz: Es handelt sich um eine Weise, Sinn zu produzieren; die Modi können potentiell für jede Art der Produktion funktionieren. Ich selbst habe mich in einem meiner ersten Beiträge zum Smartphone (Odin 2009) dazu hinreißen lassen, die unglückliche Formulierung digitaler Modus zu verwenden. Obwohl es mir beim Schreiben des Textes darum ging zu betonen, dass der Zuschauer die Bilder im Sinne des „wie wurde das gemacht?“ befragt und ich den Begriff des Modus noch nicht wirklich theoretisiert hatte, war es ein Fehler, vom digitalen Modus zu sprechen. Denn die Frage „wie wurde das gemacht?" hat einen viel größeren Anwendungsbereich als den des Digitalen - sie kann tatsächlich auf alles Mögliche bezogen werden. Daher habe ich die Formulierung korrigiert und den (oben dargelegten) Modus des Making of konstruiert.

Allerdings: Auch wenn die Modi potentiell auf jede Art der Produktion angewandt werden können, ist es doch normal, zur Charakterisierung eines Modus von einem bestimmten Produktionstyp auszugehen, für den dieser Modus besonders wichtig ist. So zeigen Fanny Georges und Nicolas Auray (2012), dass man das Phänomen der machinima $a^{5}$ nicht verstehen kann, ohne auf den Modus des Pastiche zu rekurrieren, welcher der privilegierte Modus der Fans ist und der sich durch eine Imitation (auf allen Ebenen) der diskursiven Kommunikationskompetenz charakterisiert. Ganz offensichtlich lässt sich aus

\footnotetext{
5 Das Wort machinima setzt sich zusammen aus „machine“, „cinéma“ und „animation“ und wurde 1999 von Anthony Bailey und Hugh Hancock erfunden. „Das Wort bezeichnet ein filmisches Genre und eine Produktionstechnik, bei der Videosequenzen verwendet werden, die in Echtzeit in virtuellen Räumen aufgenommen werden (meistens, aber nicht immer, von Videospielen)“, hieß es dazu auf der Videospiel-Konferenz Des cubes et des pioches 2, die 2015 in Tarbes stattfand.
} 
allem ein Pastiche machen: Es ist eine Weise, Sinn zu produzieren. Man kann daher zu Recht von einem Modus sprechen.

Einige Beiträge beziehen sich auf interaktive Produktionen, die in meinem Werk abwesend sind, zumindest wenn man darunter nur solche Produktionen versteht, die explizit eine Intervention des Rezipienten verlangen. Für die Semiopragmatik ist jede Produktion interaktiv: Der Text wird immer vom Zuschauer oder Leser konstruiert. Es ist jedoch auch wahr, dass die explizit interaktiven Produktionen sich vervielfacht haben und dass sie zu analysieren sind. Aber braucht man dafür einen besonderen Modus oder mehrere besondere Modi? Da bin ich nicht so sicher. Marianne Charbonnier und JeanMichel Denizart haben in ihrer Arbeit über Webdokus (2016) vorgeschlagen, einen webdokumentarisierenden Modus zu konstruieren, der sich durch eine Co-Enunziation von nicht nur zwei, sondern drei enunziativen Instanzen auszeichnet: Sender, Empfänger und Interface des Programms. Sicher ist es wichtig, die enunziative Rolle des Interface in diesem Kontext anzuerkennen, aber mir scheint, dass es sich hier weniger um einen neuen Modus handelt als um eine Art Dispositiv, das heißt um einen spezifischen Rahmen, innerhalb dessen die Modi wirken (in diesem Fall der dokumentarisierende Modus). $\mathrm{Zu}$ bemerken ist auch, dass das Werk von Marida Di Crosta, Entre cinéma et jeux vidéo: l'interface-film (2009), auf das sich diese Analyse stützt, Produktionen betrifft, die im fiktionalisierenden Modus funktionieren (wie hypermediale Fiktionen, interaktive Video-Erzählungen oder narrative Spiele), was zeigt, dass es sich um ein Dispositiv handelt, innerhalb dessen verschiedene Modi zur Anwendung kommen können. Tatsächlich ändern sich nicht die Modi, sondern die Tatsache, dass man es mit einem Dispositiv zu tun hat, das die physische Aktivität des Zuschauers mobilisiert: „Der Zuschauer erlangt ein neues Niveau der Intentionalität, das den Rahmen der Sinnproduktion überschreitet. Seine Begehren kann er dank der physischen Handhabung des Programms erweitern“, bemerken auch Charbonnier und Denizart (2016, 288).

In ihrer Analyse von Prison Valley (David Dufresne \& Philippe Brault, F 2009) rückt Anna Wiehl (2016) ebenfalls die Tatsache in den Vordergrund, dass der Rezipient dieser Webdoku eine „physische Handlung“ ausführt. Sie unterscheidet auf dieser Achse drei Modi, die 
drei Aktivitäten entsprechen: einen ludischen Modus, der darin besteht, zu schauen und auf dem Bildschirm zu agieren; ${ }^{6}$ einen Modus des kommunikativen Austausches, der darin besteht, vermittels des audiovisuellen Materials und über den direkten Einbezug der Teilnehmer zu interagieren; ${ }^{7}$ schließlich einen Modus der kreativen Vernetzung: Der Nutzer setzt sich über die (kollektive) Produktion neuer Inhalte auf kreative Weise mit dem dokumentarischen Material und den anderen Nutzern auseinander. Ich habe allerdings das Gefühl, dass die drei Modi nicht ganz denselben Status besitzen. Bei den ersten beiden handelt es sich (wie bei Charbonnier und Denizart) in Wirklichkeit um Dispositive: ein Dispositiv der Beziehung zum Bildschirm, ein Dispositiv der Vermittlung. Der Modus der kreativen Vernetzung scheint mir dagegen gleichzeitig einem Dispositiv (dem Netzwerk) und einem Modus zu entsprechen: dem Eingreifen eines kollektiven Enunziators, das heißt der Sinnproduktion in der Gruppe durch die Interaktion der Mitglieder und in Bezug auf das, was die Gruppe teilt. Dieser Modus der Sinnproduktion ist nicht neu und funktioniert in verschiedenen Dispositiven, etwa dem der Projektion eines Familienfilms, während der man viel spricht, um gemeinsam den Sinn des Films (den familiären Mythos) $\mathrm{zu}$ produzieren. Ich habe vorgeschlagen, ihn den privaten Modus zu nennen und seine Funktionsweise folgendermaßen beschrieben:

- auf der diskursiven Ebene: unbestimmt in seiner Form, aber in einem gegebenen Kontext ziemlich stark standardisiert;

- auf der affektiven Ebene: euphorische Affekte, Zugehörigkeitsgefühl zu einer Gemeinschaft;

- auf der enunziativen Ebene: Konstruktion eines realen, kollektiven Enunziators;

- auf der relationalen Ebene: Handlungen im Innern einer Gruppe.

6 „Im Fall von Prison Valley manifestiert sich dieser Modus wenn der Nutzer die Rolle eines Journalisten einnimmt, der auf dem Gebiet der Canon City recherchiert. Durch seine Entscheidungen folgt der Nutzer seinem Weg entlang voraufgezeichneter Interviews und kann zusätzliches Material wie Fotos, Dokumente, Statistiken etc. konsultieren.“ (ibid., 296) 7 „In dem (virtuellen) Motelzimmer findet der Nutzer einen Laptop, der ihm erlaubt, sich direkt mit anderen Nutzern über das soziale Netzwerk zu verbinden“ (ibid., 299). 
Für mich ist klar, dass sich dieser Modus auch außerhalb des familiären Kontexts anwenden lässt: Der private Modus funktioniert für alle Arten der Gemeinschaft, seien sie real (Filmclubs, Vereine, Klassenausflüge, Politaktivisten, Ortsgemeinschaften etc.) oder virtuell (vgl. Proulx/Poissant/Sénécal 2006). Fassen wir zusammen: Mein Vorschlag ist ein doppelter: 1. die Interaktivität auf eine besondere Art Kommunikationsraum zu beziehen: Räume, die explizit interaktive Dispositive einsetzen (Webdokus, Webserien, Spiele etc.); 2. den privaten Modus zu nutzen, um zu verstehen, was bei einer kollektiven Sinnproduktion geschieht.

Viele Forscher haben übrigens in ihren Arbeiten vorgeschlagen, die Semiopragmatik mit anderen Ansätzen zu verbinden: mit der Peirce'schen Semiotik, der Rhetorik, der kognitiven Semiotik der groupe $\mu$, dem Interaktionismus, soziologischen, ethnografischen, historischen Ansätzen etc. Dies erscheint mir nicht nur legitim, sondern sogar notwendig. Insbesondere ist es unausweichlich, dass sich die Semiopragmatik, die aus guten Gründen - aber inklusive des Risikos, sich von der Wirklichkeit zu entfernen - ein Top-down-Modell ist, mit empirischen Ansätzen konfrontiert sieht. So bemerkt Federica Villa (2006, 38f), dass der Zuschauer der Semiopragmatik offensichtlich kein gänzlich empirischer („non pienamente empirico“), sondern ein berechneter (,calcolato“) sei. Geraldine Poels $(2016,47)$ bekräftigt:

Die Frage der Spezifik der Zuschauerhaltung je nach Medium kann nicht allein der Semiopragmatik überlassen werden: Es ist wichtig zu untersuchen, wie sich die Beziehungen der realen und der historischen Zuschauer $\mathrm{zu}$ den verschiedenen Bildschirmen gestalten und gestaltet haben.

Ich kann nur zustimmen. Eins ist sicher: Die Semiopragmatik stellt sich nicht gegen empirische Untersuchungen, sondern verlangt nach ihnen. Nennen wir einige mögliche Verbindungen zwischen Semiopragmatik und empirischer Untersuchung:

1. Eine semiopragmatische Analyse kann Hypothesen formulieren, die durch empirische Untersuchungen zu verifizieren wären. In einer Analyse von drei Filmen, die sich auf Religion beziehen - ein Werbe- 
film der katholischen Kirche, We ARE the Catholic ChuRch (2008); ein Clip für Pepsi, Kungfu PEPSI CRUSH (2002/2003); und einer für Coca Cola light, HAVE A GREAT BREAK $(2005)^{8}$ - habe ich gezeigt, dass die Berücksichtigung der mentalen Kommunikationsräume, die die drei Filme aufrufen, eine Hypothesenbildung darüber erlaubt, in welchen Räumen die jeweiligen Kommunikationsstrategien gut (oder schlecht) funktionieren. Der erste Film mit seinem Lyrismus, seiner emphatischen oder pompösen Musik, die wirkt, als solle sie uns davontragen, seinen fiktionalisierenden Bildern mit stark affektivem Koeffizienten, seinem Loblied auf die katholische Kirche als universelle Familie, als wirkungsvolle und beruhigende Gemeinschaft gegenseitiger Hilfe, als fröhliche Gemeinschaft, deren Rituale festlichen Charakter haben - dieser Film scheint für einen Zuschauer konzipiert zu sein, dessen mentaler Kommunikationsraum der der evangelikalen Kirchen ist. Der zweite Film zeigt uns ein Initiationsritual in einem buddhistischen Tempel, der Pepsi gewidmet ist (das Symbol dieser Gemeinschaft ist der schlüssellochförmige Öffner, mit dem die Pepsi-Dose geöffnet wird). Es wäre nicht verwunderlich, wenn der Film von Mitgliedern der buddhistischen Gemeinschaft als verletzend empfunden würde, aber von anderen Zuschauern wird er wohl akzeptiert, da er nicht dazu bestimmt ist, die Religion in Frage zu stellen, sondern uns einfach mit Humor und starken filmischen Referenzen einlädt, in die Pepsi-Gemeinschaft einzutreten. Der letzte Film dagegen, in dem ein gutaussehender Mann unter dem interessierten Blick einer hübschen jungen Frau nach dem Baden aus dem Meer steigt, sich dann als Priester erweist (er zieht seinen Talar an) und der jungen Frau mit dem Schaum der Cola ein Kreuz auf die Stirn zeichnet, hatte gute Chancen, die Entrüstung der katholischen Kirche zu provozieren. Im Schlussteil des Artikels ergänzte ich, dass diese „Hypothesen durch Untersuchungen im Feld bestätigt (oder widerlegt) werden müssten“ (Odin 2015, 30). Die Tatsache, dass der letzte Film in Italien verboten wurde, kann dabei schon als Anfang der empirischen Bestätigung gesehen werden.

\footnotetext{
8 Im Workshop „Thinking methods in media and religion“, der von der Forschungsgruppe „Média et réligion“ im November 2014 in Zürich ausgerichtet wurde, waren diese drei Filme zur Analyse ausgewählt worden.
} 
2. Die Semiopragmatik bietet ein Arsenal an Fragen (etwa ausgehend von den Modi), die eine Untersuchung im Feld über die enunziativen Positionen der Kommunikationsaktanten befruchten kann. In dieser Hinsicht zeigt Julien Pequignot (2017), dass die Serie NOOB (Fabien Fournier, F 2008) zwar präsentiert wird, als solle sie im fiktionalisierenden Modus (als fiktionale Serie im fantastischen Subgenre) gelesen werden. Eine Analyse der spontanen Reaktionen der Zuschauer ergibt jedoch, dass sie vor allem im privaten Modus gelesen wird: im Gefühl der Zugehörigkeit zu einer besonderen Gemeinschaft (der Fans, Gamer, geeks etc.). In ähnlichem Sinn offenbart eine von Jean Châteauvert (2018) durchgeführte Analyse von Kommentaren auf der Facebook-Seite der Webserie Solange Te PARLE (Ina Mihalache, F 2011-) eine Sichtungserfahrung, die aus dem fiktionalisierenden Modus wegführt und eher der des Familienfilms ähnelt (intimer und privater Modus). Samuel Gantier hat sechs Monate lang eine ethnografische Untersuchung der Konzeption der Webdoku B4, FENÊTRES SUR TOUR (Jean-Christophe Ribot, F 2012) durchgeführt, in der sich zeigte, dass alles dafür unternommen wurde, den Zuschauern eine Lektüre im von ihm sogenannten „hermeneutischen Modus“ nahezulegen. (Gemeint ist eine Lektüre, in der der „Interakteur“ aufgefordert wird, das Gezeigte mit verschiedenen Ansätzen zu interpretieren: Ansätzen zu den Figuren, zum Filmtypus, zur Thematik.) Tatsächlich konstatiert Gantier nach einer Untersuchung der Rezeption jedoch, dass die Nutzer praktisch ausschließlich die Lektüre nach Filmtypen anwenden. In den drei genannten Beispielen bestätigen die empirischen Untersuchungen die Hypothese der Semiopragmatik zur Trennung zwischen dem Raum der Produktion und dem der Lektüre: Beide erzeugen jeweils eine andere Sinnproduktion.

3. Die Semiopragmatik interessiert sich für Prozesse der Sinnproduktion (die Modi), sagt jedoch nichts über die aktivierten Inhalte. Die Untersuchung im Feld kann also die theoretische Analyse anreichern und mit Gehalt füllen. Giuseppina Sapio, die in ihrer Dissertation La pratique des home movies den semiopragmatischen mit dem interaktionistischen Ansatz von Erving Goffman verbindet, hat dies verstanden: 
Die Kooperation der beiden Ansätze erscheint uns sehr wirkungsvoll, denn wenn wir auf der einen Seite einen kommunikationstheoretischen Rahmen bemühen, der den Kontext berücksichtigt und die Modalitäten der Interaktion zwischen den Aktanten erklärt (die Semiopragmatik), hoffen wir andererseits, dieses heuristische Schema „füllen“ zu können, indem wir die tatsächlichen Interaktionen zwischen den Akteuren und ihre symbolische Bedeutung analysieren (mithilfe des dramaturgischen und interaktionistischen Ansatzes) (Sapio 2015, 29).

4. Frank Kessler hat vorgeschlagen, das Modell selbst zu historisieren: „Die Beziehung, die sich zwischen einer Technologie, einer filmischen Form mit ihrer Art der Adressierung und der daraus resultierenden Positionierung des Zuschauers ergibt, lässt sich historisieren“ (2011, 23). Als „historische Pragmatik“ bezeichnet er seinen Ansatz, der es erlaubt, „das Konzept des Dispositivs, im Sinne einer historischen Konfiguration, als heuristisches Instrument $\mathrm{zu}$ benutzen, um die Funktion und die Funktionsweise eines Mediums zu verschiedenen Zeitpunkten seiner Geschichte zu erforschen“ (ibid.). Ich bin diesem Vorschlag in meinem Aufsatz „Amateur Technologies of Memory, Dispositifs and Communication Spaces“ (2018) gefolgt. Dort bin ich der Evolution der Dispositive der Produktion von Familienfilmen nachgegangen, vom Raum des Familienfilms über den des Videos bis zum heutigen digitalen Raum.

5. Abschließend möchte ich - im Zuge des corporeal turn (vgl. Sheets-Johnstone 2009; Jullier 2018; Eugenie 2019) - die notwendige Verbindung zur empirischen Kognitionspsychologie betonen (Coëgnarts/Kravanja 2015; Grabowski 2015; Nannicelli/Taberham 2014; Zacks 2015). In Kommunikationsräume habe ich diesem Ansatz zwar auch schon einen Platz eingeräumt, ihn jedoch auf die universellen Bedingungen beschränkt: jene der „Vorverkabelung“ unseres kognitiven Wahrnehmungsapparates (Bedingungen, die im Kino etwa die Wahrnehmung von Bewegung, die Rekonstruktion der Raumtiefe oder das Abtasten des Bildes durch die Augen regeln) und jene unseres narrativen Begehrens. Die Beziehung zum Körper betrifft jedoch keineswegs nur die universellen Bedingungen; in semiopragmatischer 
Perspektive wäre es interessant, die spezifischen körperlichen Bedingungen der jeweiligen Kommunikationsräume zu berücksichtigen (vgl. Bellour 2012).

Es wären noch viele andere Fragen zu nennen, eins ist jedoch sicher: Für mich ist es eine große Befriedigung zu sehen, dass sich einige Forscher des Modells bedienen (ohne es einfach anzuwenden), dass sie es sich aneignen, es weiterentwickeln oder davon ausgehen, um anderswo zu landen. Ich kann mir nur wünschen, dass sich diese Entwicklung fortsetzt.

Paris, Dezember 2018 


\section{Literatur}

Bellour, Raymond (2012): La querelle des dispositifs: Cinéma - installations, expositions. Paris: P.O.L.

Casetti Francesco / Sampietro Sara (2012): With Eyes, With Hands. The Relocation of Cinema into iPhone. In: Pelle Snickars \& Patrick Vonderau (Hg.), Moving Data: The iPhone and the Future of Media. New York [etc.]: Columbia University Press, S. 19-32.

Charbonnier, Marianne / Denizart, Jean-Michel (2016) : Webdocumentaire et spectateur mutant. Le cas de PEAU D’ÂNE, DEMY ET LE MERVEILleuX - produit par la Cinémathèque française. In: Jean Châteauvert \& Gilles Delavaud (Hg.), D'un écran à l'autre, les mutations du spectateur. Paris: L’Harmatan \& INA, S. 275-291.

Châteauvert, Jean (2018): Vis-à-vis: quand Solange te parle. In: Claire Chatelet, Amanda Rueda \& Julie Savelli (Hg.), Formes audiovisuelles connectées. Aix/Marseille: Presses Universitaires, S. 116-130.

Coëgnarts, Maarten / Kravanja, Peter (Hg.) (2015): Embodied Cognition and Cinema. Leuven: Leuven University Press.

Di Crosta, Marida (2009): Entre cinéma et jeux vidéo: l'interface-film. Brüssel \& Paris: De Boeck/INA.

Eugenie, Ruggero (2019): La neurofilmologie. Une théorie pragmatique de l'audiovisuel en dialogue avec les sciences neurocognitives. In: Interrogations 27 (online) [https://www.revue-interrogations.org/La-neurofilmologie-Une-theorie].

Gantier, Samuel (2018): Évaluation du design d'expérience utilisateur d'un webdocumentaire. In: Claire Chatelet, Amanda Rueda \& Julie Savelli (Hg.), Formes audiovisuelles connectées. Aix/Marseille: Presses Universitaires, S. 131-148.

Georges, Fanny / Auray, Nicolas (2012): Approche sémiopragmatique de l'espace de communication des machinima. In: Revue des Interactions Humaines Médiatisées / Journal of Human Mediated Interactions 13,1, S. 3-36.

Grabowski, Michael J. (Hg.) (2015): Neuroscience and Media: New Understandings and Representations. London \& New York: Routledge.

Hockney, David (2001): Secret Knowledge: Rediscovering the Lost Techniques of the Old Masters. London: Thames \& Hudson.

Jullier, Laurent (2018): Cinéma et psychologie cognitive: le „tournant corporel“ des études audiovisuelles. In: Ekphrasis 20, 2 („Cinema, cognition and art“, hg. v. Mircea Deaca), S. 20-39 [http://ekphrasisjournal.ro/docs/R1/20E2.pdf].

Kessler, Frank (2011): Recadrages: pour une pragmatique historique du dispositif cinématographique. In: Recherches sémiotiques 31,1-2-3, S. 15-32.

Lanbach, Claudia (2018): Le cinéma de poche, les enjeux et les usages (2005-2015). Diss. Université de Paris III - Sorbonne Nouvelle.

Loureau, René (1970): L'analyse institutionnelle. Paris: Minuit.

Metz, Christian (2017): Daniel Pecheron \& Marc Vernet: Interview with Christian Metz [frz. 1975], in: Warren Buckland \& Daniel Fairfax (Hg.), Conversations with Christian Metz Selected Interviews on Film Theory (1970-1991). Amsterdam: Amsterdam University Press, S. 109-143.

Nannicelli, Ted / Taberham, Paul (Hg.) (2014): Cognitive Media Theory. New York \& London: Routledge. 


\section{Literatur}

Odin, Roger (1999): La question de l'amateur. In: Communications 68 („Le cinéma en amateur"), S. 47-90.

— (2009): Le „Pocket Film Spectateur“. In: Festival Pocket Films [http://www.festivalpocketfilms.fr/spip.php?article1114].

- (2012): Cinéma et téléphone portable. Approche sémio-pragmatique. In: Théorème 15 („Le cinéma en situation“, hg. v. Laurent Creton, Laurent Jullier \& Raphaëlle Moine), S. 79-88.

- (2015): Religion and Spaces of Communication. In: JRFM - Journal for Religion, Film and Media 1,1 („Thinking Methods in Media and Religion“, hg. v. Marie-Therese Mäder, Daria Pezzoli-Olgiati \& Christian Wessely), S. 23-30.

- (2016a): The Concept of the Mental Screen: The Internalized Screen, the Dream Screen, and the Constructed Screen. In: José Moure \& Dominique Château (Hg.), Screens: From Materiality to Spectatorship - A Historical and Theoretical Reassessmenteds. Amsterdam: Amsterdam University Press, S. 176-186.

- (2016b): Espaces de communication physiques, espaces de communication mentaux. In: Jean Châteauvert \& Gilles Delavaud (Hg.), D’un écran à l'autre: les mutations du spectateur. Paris: INA \& L'Harmattan, S. 331-343.

- (2018): Amateur Technologies of Memory, Dispositifs and Communication Spaces. In: Susan Aasman, Andreas Fickers \& Joseph Wachelder (Hg.), Materializing Memories: Dispositifs, Generations, Amateurs. New York: Bloomsbury, S. 19-35.

- (2019) Special Effects and Spaces of Communication: A Semio-pragmatic Approach. In: Marc Furstenau \& Martin Lefebvre (Hg.), Theorizing Special Effects in Film History. Amsterdam: Amsterdam University Press [i. Ersch.].

Péquignot, Julien (2017): Comment faire une enquête sémio-pragmatique? Les publics des web-séries et leurs discours „spontanés“. In: Interrogations 24 [https://www.revueinterrogations.org/De-Peirce-a-Odin-tenants-et].

Poels, Géraldine (2016): Le téléspectateur, un ciné-spectateur comme les autres? Les apports de l'histoire de la réception télévisuelle. In: Jean Châteauvert \& Gilles Delavaud (Hg.), D’un écran à l'autre, les mutations du spectateur. Paris: L'Harmatan \& INA, S. 3749.

Proulx, Serge / Poissant, Louise / Sénécal, Michel (2006): Communautés virtuelles: penser et agir en réseau. Québec: Presses de l'université Laval.

Sapio, Giuseppina (2015): La pratique des home movies. Culture audiovisuelle et genèse de la méta-famille. Diss. Université de Paris III - Sorbonne Nouvelle.

Savigneau, Josyane (2009): Exit le fantôme, de Philip Roth: last exit pour Zuckerman. In: Le monde (08.10.2009) [https://www.lemonde.fr/livres/article/2009/10/08/exit-le-fantomede-philip-roth_1250888_3260.html].

Sheets-Johnstone, Maxine (2009): The Corporeal Turn: An Interdisciplinary Reader. Exeter: Imprint Academic.

Villa, Federica (2006): Oltre la semiotica. Testo e contest. In: Paolo Bertetto (Hg.), Metodologie di analisi del film. Rom/Bari: Laterza, S. 26-58.

Wiehl, Anna (2016): D’une relation ludique? Quand le cinéma se remet en jeu. In: Jean Châteauvert \& Gilles Delavaud (Hg.), D’un écran à l'autre, les mutations du spectateur. Paris: L'Harmatan \& INA, S. 291-306.

Zacks, Jeffrey M. (2015): Flicker: Your Brain on Movies. Oxford \& New York: Oxford University Press. 